\title{
Stereospecific Rhodium-Catalyzed Allylic Substitution with Alkenyl Cyanohydrin Pronucleophiles: Construction of Acyclic Quaternary Substituted $\alpha, \beta$-Unsaturated Ketones
}

\author{
Ben W. H. Turnbull, ${ }^{\dagger}$ Samuel Oliver ${ }^{\ddagger}$ and P. Andrew Evans ${ }^{*}{ }^{\dagger}$ \\ † Department of Chemistry, Queen's University, 90 Bader Lane, Kingston, ON, K7L 3N6, Canada \\ ${ }^{\ddagger}$ Department of Chemistry, The University of Liverpool, Crown Street, Liverpool, L69 7ZD, UK
}

\section{Contents of Supporting Information:}

1. General Information S1

2. Spectral Data for the Alkenyl Cyanohydrins 2a-r $\quad$ S2

3. Representative Experimental Procedure for the Rhodium-Catalyzed Allylic Substitution S9 with Alkenyl Cyanohydrin Pronucleophiles

4. Spectral Data for the $\alpha, \beta$-Unsaturated Ketone Products 3a-x $\quad$ S10

5. Spectral Data for the Enantiomerically Enriched Tertiary Allylic Alcohols 5a-c S21

6. Representative Experimental Procedure for the Stereospecific Rhodium-Catalyzed S22 Allylic Substitution with Alkenyl Cyanohydrin Pronucleophiles

7. Spectral Data for the Enantiomerically Enriched Ketone Products 6a-c S23

$\begin{array}{lll}\text { 8. Preparation of } \alpha, \alpha^{\prime} \text {-Dialkyl Ketone } 9 & \text { S25 }\end{array}$

$\begin{array}{lll}\text { 9. Proof of Configuration/Preparation of LY426965 } & \text { S27 }\end{array}$

10. References $\quad$ S31

11. Proton and Carbon NMR Spectra $\quad$ S32

$\begin{array}{lr}\text { 12. HPLC Chromatograms } & \text { S140 }\end{array}$

\section{General Information}

All reactions were carried out under an argon atmosphere in anhydrous solvent using commercially available reagents that were purchased and used as received. The tertiary allylic carbonates 1a-h were prepared according to the previously reported procedure. ${ }^{1 b}(R)$-Linalool $(99: 1$ er) was purchased from Aldrich. Tetrahydrofuran was freshly distilled from sodium benzophenone ketyl. 
Analytical thin layer chromatography (TLC) was performed on pre-coated $0.25 \mathrm{~mm}$ thick silica gel $60-\mathrm{F}_{254}$ plates (Merck or Whatman PE SIL G/UV); visualized using UV light and by treatment with a $\mathrm{KMnO}_{4}$, followed by heating. All compounds were purified by flash chromatography using silica gel 60 (40-63 $\mu \mathrm{m}$, Silicycle or FluoroChem) and gave spectroscopic data consistent with being $\geq 95 \%$ the assigned structure. Melting points (uncorrected) were obtained from a Büchi M560 melting point instrument. Optical rotations $\left([\alpha]_{\mathrm{D}}^{\circ \mathrm{C}}\right)$ were measured on a Anton Parr MCP 200 polarimeter with a tungsten halogen lamp $(589 \mathrm{~nm})$ at the stated temperature (indicated in ${ }^{\circ} \mathrm{C}$ as superscript) using a 0.7 $\mathrm{mL}$ quartz cell of $100 \mathrm{~mm}$ length; solution concentrations $(c)$ are given in $\mathrm{g} / 100 \mathrm{~mL}$. ${ }^{1} \mathrm{H}$ NMR and ${ }^{13} \mathrm{C}$ NMR spectra were recorded on a Bruker Avance DRX-500 spectrometer in $\mathrm{CDCl}_{3}$ at ambient temperature; chemical shifts $(\delta)$ are given in ppm and calibrated using the signal of residual undeuterated solvent as internal reference $\left(\delta_{\mathrm{H}}=7.26 \mathrm{ppm}\right.$ and $\left.\delta_{\mathrm{C}}=77.16 \mathrm{ppm}\right)$. ${ }^{1} \mathrm{H}$ NMR data are reported as follows: chemical shift (multiplicity, $1^{\text {st }}$ order spin system if available, coupling constant, integration). Coupling constants $(J)$ are reported in $\mathrm{Hz}$ and apparent splitting patterns are designated using the following abbreviations: s (singlet), $\mathrm{d}$ (doublet), $\mathrm{t}$ (triplet), $\mathrm{q}$ (quartet), $\mathrm{m}$ (multiplet), br (broad), app. (apparent) and the appropriate combinations. ${ }^{13} \mathrm{C}$ NMR spectra with complete proton decoupling were described with the aid of an APT sequence, separating methylene and quaternary carbons (e, even), from methyl and methine carbons (o, odd). IR spectra were recorded on an Agilent Technologies Cary 630 FT-IR (ATR) or Perkin-Elmer FT-IR Spectrum 100 (ATR) spectrometer; wavenumbers $(v)$ are given in $\mathrm{cm}^{-1}$; and the abbreviations $\mathrm{w}$ (weak, <33\%), $\mathrm{m}$ (medium, 33-66\%), s (strong, $>66 \%$ ), vs (very strong, $\geq 95 \%$ ) and br (broad) are used to describe the relative intensities of the IR absorbance bands. Mass spectra were obtained through the University of Liverpool Mass Spectrometry Center, the EPSRC National Mass Spectrometry Service Center or Queen's University Mass Spectrometry and Proteomics Services Unit. All liquid chromatographs were obtained on an Agilent 1200 series or an Agilent 1260 Infinity series HPLC equipped with a variable wavelength UV detector. The instrument was fitted with a CHIRALCEL ${ }^{\mathrm{TM}}$ OJ-H column (Diacel, $4.6 \mathrm{~mm} \times 25 \mathrm{~cm}$ ) or CHIRALCEL OD ${ }^{\mathrm{TM}}$ column (Diacel, $4.6 \mathrm{~mm} \times 25 \mathrm{~cm}$ ).

\section{Spectral Data for the Alkenyl Cyanohydrins 2a-q}

The alkenyl cyanohydrins $\mathbf{2 a - q}$ were prepared from the corresponding $\alpha, \beta$-unsaturated aldehydes according to the previously reported procedure. ${ }^{1}$ When not commercially available, the required $\alpha, \beta-$ unsaturated aldehydes were prepared in three steps according to the procedure reported by Das. ${ }^{2}$ 


\section{(E)-2-((tert-Butyldimethylsilyl)oxy)-6-phenylhex-3-enenitrile (2a)}

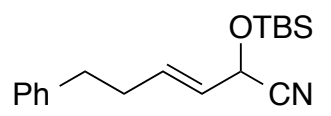

Color and State: Colorless oil.

${ }^{1}$ H NMR $\left(500 \mathrm{MHz}, \mathrm{CDCl}_{3}\right) \delta$ 7.30-7.27 (m, 2H), 7.21-7.16 (m, 3H), 5.97 (dtd, $J=15.0,6.8,1.4 \mathrm{~Hz}, 1 \mathrm{H}$ ), 5.54 (ddt, $J=15.3,5.9,1.5 \mathrm{~Hz}, 1 \mathrm{H}$ ), 4.89 (app. dq, $J=5.9,1.0 \mathrm{~Hz}, 1 \mathrm{H}$ ), $2.73(\mathrm{t}, J=7.7 \mathrm{~Hz}, 2 \mathrm{H}), 2.44-2.39(\mathrm{~m}, 2 \mathrm{H}), 0.91$ (s, 9H), 0.16 (s, 3H), $0.12(\mathrm{~s}, 3 \mathrm{H})$.

${ }^{13}$ C NMR (125 MHz, CDCl $) \delta 141.16$ (e), 134.84 (o), 128.55 (o), 128.53 (o), 126.19 (o), 125.83 (o), 118.89 (e), 62.63 (o), 35.11 (e), 33.65 (e), 25.67 (o), 18.27 (e), -4.89 (o), -4.92 (o).

IR (Neat) 3028 (w), 2955 (w), 2930 (m), 2858 (w), 1669 (w), 1604 (w), 1472 (w), 1254 (m), 1108 (m), 1063 (m), 967 (m), 837 (vs), 780 (s) $\mathrm{cm}^{-1}$.

HRMS (ESI $[\mathrm{M}+\mathrm{Na}]^{+}$) calcd for $\mathrm{C}_{18} \mathrm{H}_{27} \mathrm{NNaOSi} 324.1760$, found 324.1762 .

\section{(Z)-2-((tert-Butyldimethylsilyl)oxy)-6-phenylhex-3-enenitrile (Z-2a)}

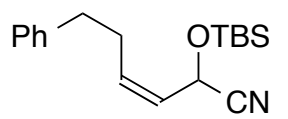

Color and State: Colorless oil; $E / Z=1: 7$

${ }^{1}$ H NMR $\left(500 \mathrm{MHz}, \mathrm{CDCl}_{3}\right) \delta$ 7.32-7.27 (m, 2H), 7.23-7.16 (m, 3H), $5.71(\mathrm{dtd}, J$ $=10.7,7.5,1.2 \mathrm{~Hz}, 1 \mathrm{H}), 5.54$ (ddt, $J=10.8,7.9,1.5 \mathrm{~Hz}, 1 \mathrm{H}), 5.06$ (dd, $J=8.1,1.2 \mathrm{~Hz}, 1 \mathrm{H}), 2.73$ (td, $J=7.7,1.9 \mathrm{~Hz}, 2 \mathrm{H}), 2.46-2.41$ (m, 2H), 0.89 (s, 9H), 0.15 (s, 3H), 0.11 (s, 3H).

${ }^{13}$ C NMR (125 MHz, $\mathrm{CDCl}_{3}$ ) $\delta 140.82$ (e), 134.26 (o), 128.64 (o), 128.44 (o), 126.37 (o), 126.36 (o), 119.06 (e), 58.08 (o), 35.19 (e), 29.83 (e), 25.58 (o), 18.12 (e), -4.93 (o), -4.95 (o).

IR (Neat) 3029 (w), 2952 (w), 2930 (w), 2859 (w), 1658 (w), 1605 (w), 1473 (w), 1255 (m), 1101 (s), $971(\mathrm{w}), 837(\mathrm{vs}), 780(\mathrm{~s}) \mathrm{cm}^{-1}$.

HRMS (ESI $[\mathrm{M}+\mathrm{H}]^{+}$) calcd for $\mathrm{C}_{18} \mathrm{H}_{28} \mathrm{NOSi} 302.1935$, found 302.1931 .

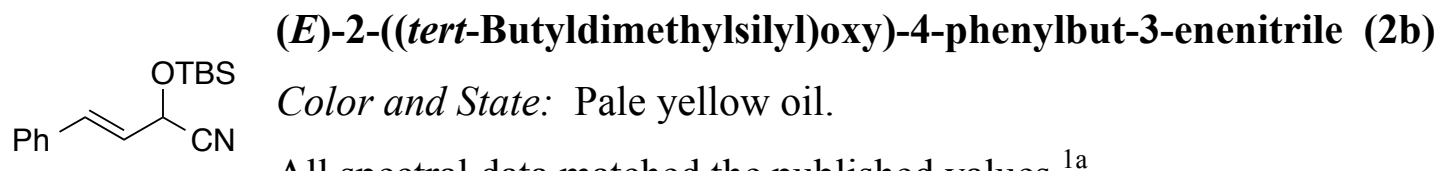

All spectral data matched the published values. ${ }^{1 \mathrm{a}}$

${ }^{1}$ H NMR $\left(500 \mathrm{MHz}, \mathrm{CDCl}_{3}\right) \delta$ 7.42-7.41 (m, 2H), 7.38-7.34 (m, 2H), 7.33-7.30 (m, 1H), $6.81(\mathrm{dd}, J$ $=15.7,1.1 \mathrm{~Hz}, 1 \mathrm{H}), 6.19(\mathrm{dd}, J=15.8,5.8 \mathrm{~Hz}, 1 \mathrm{H}), 5.14(\mathrm{dd}, J=5.8,1.4 \mathrm{~Hz}, 1 \mathrm{H}), 0.95(\mathrm{~s}, 9 \mathrm{H}), 0.23$ (s, 3H), $0.20(\mathrm{~s}, 3 \mathrm{H})$.

IR (Neat) 3029 (w), 2955 (m), 2931 (m), 2859 (m), 1654 (w), 1579 (w), 1472 (m), 1255 (m), 1101 (m), $1079(\mathrm{~m}), 966(\mathrm{~m}), 831(\mathrm{~s}), 781(\mathrm{~s}) \mathrm{cm}^{-1}$. 


\section{OTBS (E)-2-((tert-Butyldimethylsilyl)oxy)pent-3-enenitrile (2c)

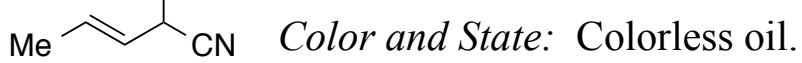

${ }^{1}$ H NMR $\left(500 \mathrm{MHz}, \mathrm{CDCl}_{3}\right) \delta 5.95(\mathrm{dqd}, J=15.1,6.7,1.5 \mathrm{~Hz}, 1 \mathrm{H}), 5.54(\mathrm{ddq}, J=15.2,6.0,1.7 \mathrm{~Hz}$, 1H), 4.89 (app. d pentet, $J=6.0,1.1 \mathrm{~Hz}, 1 \mathrm{H}), 1.76$ (ddd, $J=6.6,1.6,1.1 \mathrm{~Hz}, 3 \mathrm{H}), 0.91$ (s, 9H), 0.17 $(\mathrm{s}, 3 \mathrm{H}), 0.14(\mathrm{~s}, 3 \mathrm{H})$.

${ }^{13}$ C NMR (125 MHz, $\left.\mathrm{CDCl}_{3}\right) \delta 130.82$ (o), 126.33 (o), 118.92 (e), 62.62 (o), 25.61 (o), 18.21 (e), 17.51 (o), -4.95 (o), -4.98 (o).

IR (Neat) 2956 (w), 2931 (w), 2859 (w), 1680 (w), 1615 (w), 1472 (w), 1255 (m), 1088 (s), 837 (vs), $781(\mathrm{~s}) \mathrm{cm}^{-1}$.

HRMS (ESI $\left[\mathrm{M}+\mathrm{NH}_{4}\right]^{+}$) calcd for $\mathrm{C}_{11} \mathrm{H}_{25} \mathrm{~N}_{2} \mathrm{OSi} 229.1731$, found 229.1728.

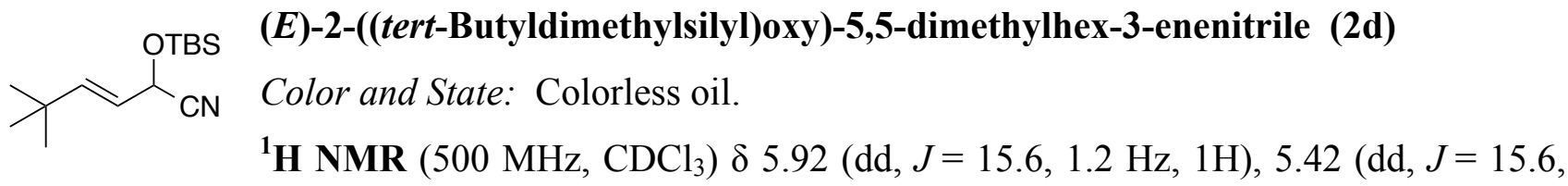

$6.2 \mathrm{~Hz}, 1 \mathrm{H}), 4.91(\mathrm{dd}, J=6.1,1.1 \mathrm{~Hz}, 1 \mathrm{H}), 1.04(\mathrm{~s}, 9 \mathrm{H}), 0.91(\mathrm{~s}, 9 \mathrm{H}), 0.16(\mathrm{~s}, 3 \mathrm{H}), 0.14(\mathrm{~s}, 3 \mathrm{H})$.

${ }^{13}$ C NMR (125 MHz, $\mathrm{CDCl}_{3}$ ) $\delta 146.60$ (o), 120.61 (o), 119.15 (e), 63.20 (o), 33.22 (e), 29.14 (o), 25.68 (o), 18.28 (e), -4.73 (o).

IR (Neat) 2958 (m), 2932 (m), 2861 (w), 1665 (w), 1473 (w), 1364 (w), 1255 (m), 1108 (m), 1071 (m), $973(\mathrm{~m}), 838(\mathrm{~s}), 779(\mathrm{~s}) \mathrm{cm}^{-1}$.

HRMS (ESI $\left[\mathrm{M}+\mathrm{NH}_{4}\right]^{+}$) calcd for $\mathrm{C}_{14} \mathrm{H}_{31} \mathrm{~N}_{2} \mathrm{OSi} 271.2200$, found 271.2204.

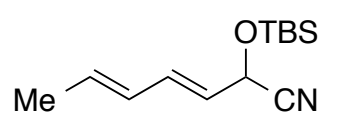

\section{(3E,5E)-2-((tert-Butyldimethylsilyl)oxy)hepta-3,5-dienenitrile (2e)}

Color and State: Colorless oil.

The product was isolated as a 6:1 mixture of $(3 E, 5 E)$ and $(3 E, 5 Z)$ isomers. Data is provided for the major isomer.

${ }^{1}$ H NMR $\left(500 \mathrm{MHz}, \mathrm{CDCl}_{3}\right) \delta 6.38(\mathrm{dd}, J=15.1,10.5 \mathrm{~Hz}, 1 \mathrm{H}), 6.09-6.04(\mathrm{~m}, 1 \mathrm{H}), 5.86(\mathrm{dq}, J=$ 14.6, 7.1 Hz, 1H), 5.54 (dd, $J=15.2,6.1 \mathrm{~Hz}, 1 \mathrm{H}), 4.97$ (d, $J=6.0 \mathrm{~Hz}, 1 \mathrm{H}), 1.79$ (d, $J=6.7 \mathrm{~Hz}, 3 \mathrm{H})$, $0.91(\mathrm{~s}, 9 \mathrm{H}), 0.17(\mathrm{~s}, 3 \mathrm{H}), 0.15(\mathrm{~s}, 3 \mathrm{H})$.

${ }^{13}$ C NMR (125 MHz, $\mathrm{CDCl}_{3}$ ) $\delta 134.12$ (o), 133.60 (o), 129.51 (o), 124.22 (o), 118.72 (e), 62.56 (o), 25.64 (o), 18.32 (o), 18.25 (e), -4.88 (o), -4.92 (o).

IR (Neat) 3025 (w), 2956 (w), 2931 (m), 2859 (w), 1661 (w), 1472 (w), 1255 (m), 1113 (m), 1080 (s), 987 (s), 837 (vs), $781(\mathrm{~s}) \mathrm{cm}^{-1}$. 
HRMS (ESI $\left[\mathrm{M}+\mathrm{NH}_{4}\right]^{+}$) calcd for $\mathrm{C}_{13} \mathrm{H}_{27} \mathrm{~N}_{2} \mathrm{OSi} 255.1887$, found 255.1885 .

\section{OTBS 2-((tert-Butyldimethylsilyl)oxy)-4-methylpent-3-enenitrile (2f) \\ CN Color and State: Colorless oil.}

${ }^{1} \mathbf{H}$ NMR $\left(500 \mathrm{MHz}, \mathrm{CDCl}_{3}\right) \delta 5.34-5.32(\mathrm{~m}, 1 \mathrm{H}), 5.08(\mathrm{~d}, J=8.4 \mathrm{~Hz}, 1 \mathrm{H}), 1.77(\mathrm{~s}, 3 \mathrm{H}), 1.72(\mathrm{~d}, J=$ $0.9 \mathrm{~Hz}, 3 \mathrm{H}), 0.90$ (s, 9H), 0.16 (s, 3H), 0.13 (s, 3H).

${ }^{13}$ C NMR (125 MHz, CDCl $) \delta 139.05$ (e), 121.27 (o), 119.58 (e), 59.07 (o), 25.62 (o), 18.45 (o), 18.16 (e), -4.85 (o), -4.88 (o).

IR (Neat) 2957 (w), 2932 (w), 2859 (w), 1673 (w), 1473 (w), 1254 (m), 1122 (m), 1073 (s), 837 (s), $779(\mathrm{~s}) \mathrm{cm}^{-1}$.

HRMS (ESI $\left[\mathrm{M}+\mathrm{NH}_{4}\right]^{+}$) calcd for $\mathrm{C}_{12} \mathrm{H}_{27} \mathrm{~N}_{2} \mathrm{OSi} 243.1887$, found 243.1883 .

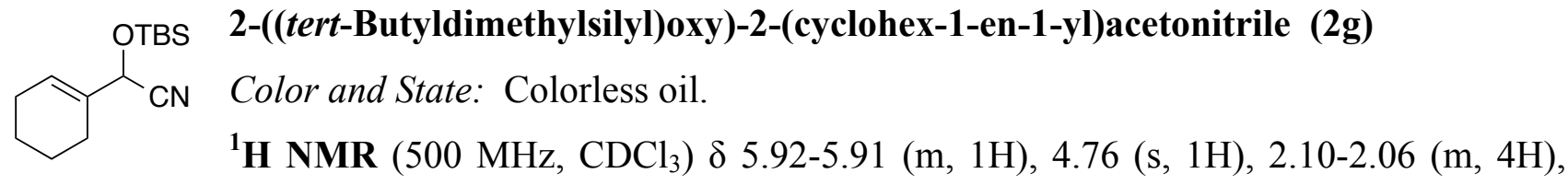
1.73-1.57 (m, 4H), 0.91 (s, 9H), 0.18 (s, 3H), 0.13 (s, 3H).

${ }^{13}$ C NMR (125 MHz, $\mathrm{CDCl}_{3}$ ) $\delta 133.54$ (e), 126.96 (o), 119.00 (e), 66.53 (o), 25.62 (o), 25.00 (e), 23.86 (e), 22.23 (e), 22.03 (e), 18.22 (e), -5.11 (o).

IR (Neat) 2930 (m), 2859 (m), 1670 (w), 1472 (w), 1254 (m), 1157 (m), 1090 (s), 1072 (s), 835 (vs), $780(\mathrm{~s}) \mathrm{cm}^{-1}$.

HRMS (ESI $\left[\mathrm{M}+\mathrm{NH}_{4}\right]^{+}$) calcd for $\mathrm{C}_{14} \mathrm{H}_{29} \mathrm{~N}_{2} \mathrm{OSi} 269.2044$, found 269.2043 .

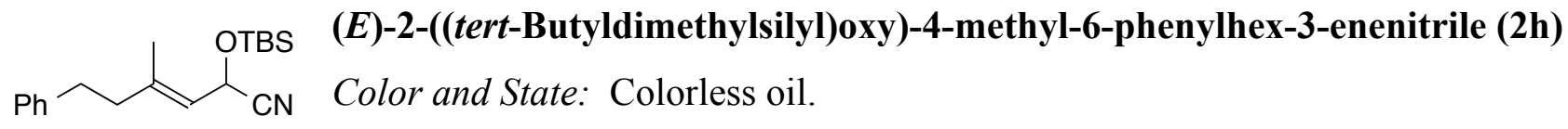

${ }^{1}$ H NMR $\left(500 \mathrm{MHz}, \mathrm{CDCl}_{3}\right) \delta$ 7.30-7.27 (m, 2H), 7.21-7.16 (m, 3H), 5.33 (app. d sextet, $J=8.1,1.3$ $\mathrm{Hz}, 1 \mathrm{H}), 5.09$ (d, $J=8.1 \mathrm{~Hz}, 1 \mathrm{H}), 2.75$ (t, $J=7.9 \mathrm{~Hz}, 2 \mathrm{H}), 2.35$ (t, $J=7.9 \mathrm{~Hz}, 2 \mathrm{H}), 1.76$ (d, $J=1.2$ $\mathrm{Hz}, 3 \mathrm{H}), 0.90$ (s, 9H), 0.15 (s, 3H), 0.11 (s, 3H).

${ }^{13}$ C NMR (125 MHz, CDCl $) \delta 141.80$ (e), 141.32 (e), 128.55 (o), 128.44 (o), 126.16 (o), 121.66 (o), 119.41 (e), 59.07 (o), 41.00 (e), 34.03 (e), 25.65 (o), 18.19 (e), 17.13 (o), -4.84 (o), -4.86 (o).

IR (Neat) 3028 (w), 2930 (m), 2858 (w), 1666 (w), 1604 (w), 1473 (w), 1254 (m), 1114 (m), 1068 (s), 837 (vs), $780(\mathrm{~s}) \mathrm{cm}^{-1}$.

HRMS (EI, $\mathrm{M}^{+}$) calcd for $\mathrm{C}_{19} \mathrm{H}_{29} \mathrm{NOSi} 315.2018$, found 315.2006. 
<smiles>CC(CCc1ccccc1)=C(C)C([Mg])C#N</smiles>

(E)-2-((tert-Butyldimethylsilyl)oxy)-3,4-dimethyl-6-phenylhex-3-enenitrile (2i)

Color and State: Colorless oil.

${ }^{1}$ H NMR $\left(500 \mathrm{MHz} \mathrm{CDCl}_{3}\right) \delta$ 7.31-7.28 (m, 2H), 7.23-7.17 (m, 3H), $5.32(\mathrm{~s}, 1 \mathrm{H}), 2.71(\mathrm{t}, J=7.9$ $\mathrm{Hz}, 2 \mathrm{H}), 2.39$ (t, $J=7.9 \mathrm{~Hz}, 2 \mathrm{H}), 1.77$ (app q, $J=1.3 \mathrm{~Hz}, 3 \mathrm{H}), 1.71-1.70(\mathrm{~m}, 3 \mathrm{H}), 0.93$ (s, 9H), 0.19 $(\mathrm{s}, 3 \mathrm{H}), 0.12(\mathrm{~s}, 3 \mathrm{H})$.

${ }^{13}$ C NMR (125 MHz, $\left.\mathrm{CDCl}_{3}\right) \delta 141.50$ (e), 134.09 (e), 128.51 (o), 128.49 (o), 126.12 (o), 126.01 (e), 119.35 (e), 62.00 (o), 37.15 (e), 33.79 (e), 25.61 (o), 18.42 (o), 18.18 (e), 12.68 (o), -5.06 (o), -5.08 (o).

IR (Neat) 3028 (w), 2954 (w), 2931 (m), 2859 (w), 1604 (w), 1473 (w), 1254 (m), 1156 (m), 1068 (s), $835(\mathrm{vs}), 778(\mathrm{~s}) \mathrm{cm}^{-1}$.

HRMS (EI, $\mathrm{M}^{+}$) calcd for $\mathrm{C}_{20} \mathrm{H}_{31} \mathrm{NOSi} 329.2175$, found 329.2170.

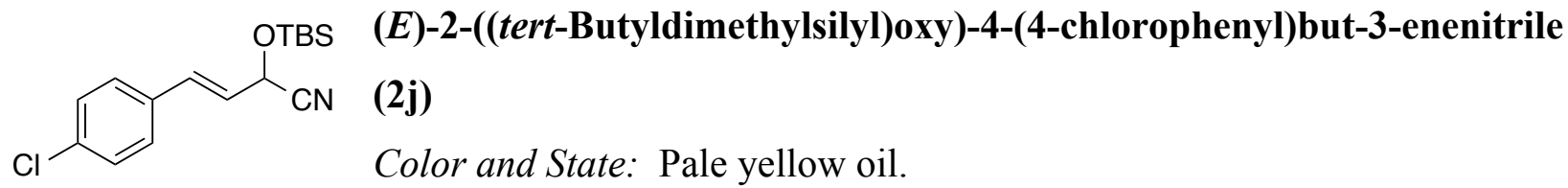

${ }^{1}$ H NMR $\left(500 \mathrm{MHz}, \mathrm{CDCl}_{3}\right) \delta$ 7.35-7.31 (m, 4H), $6.77(\mathrm{dd}, J=15.8,1.5 \mathrm{~Hz}, 1 \mathrm{H}), 6.16(\mathrm{dd}, J=15.8$, $5.6 \mathrm{~Hz}, 1 \mathrm{H}), 5.13(\mathrm{dd}, J=5.6,1.5 \mathrm{~Hz}, 1 \mathrm{H}), 0.94(\mathrm{~s}, 9 \mathrm{H}), 0.22(\mathrm{~s}, 3 \mathrm{H}), 0.19$ (s, 3H).

${ }^{13}$ C NMR (125 MHz, $\mathrm{CDCl}_{3}$ ) $\delta 134.64$ (e), 133.73 (e), 132.41 (o), 129.09 (o), 128.30 (o), 124.46 (o), 118.42 (e), 62.57 (o), 25.68 (o), 18.32 (e), -4.84 (o), -4.92 (o).

IR (Neat) 2955 (w), 2931 (w), 2859 (w), 1655 (w), 1595 (w), 1492 (m), 1255 (m), 1135 (m), 1089 (s), $967(\mathrm{~m}), 836(\mathrm{~s}), 780(\mathrm{~s}) \mathrm{cm}^{-1}$.

HRMS (ESI [M+Na $]^{+}$) calcd for $\mathrm{C}_{16} \mathrm{H}_{22}{ }^{35} \mathrm{CINNaOSi} 330.1057$, found 330.1045.

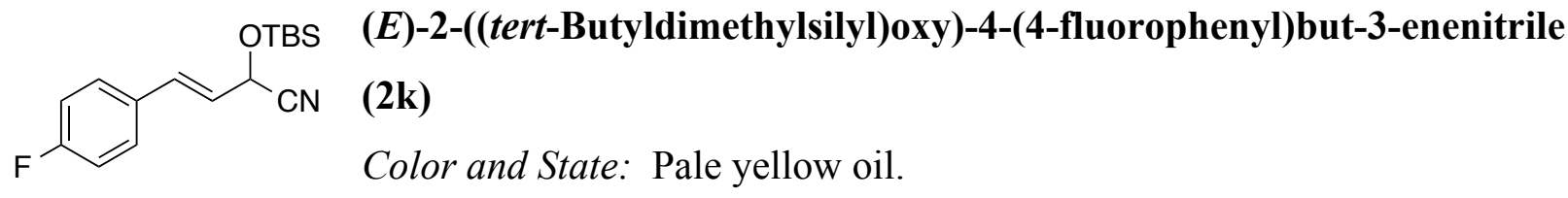

${ }^{1}$ H NMR $\left(500 \mathrm{MHz}, \mathrm{CDCl}_{3}\right) \delta$ 7.40-7.37 (m, 2H), 7.06-7.03 (m, 2H), $6.78(\mathrm{~d}, J=15.8 \mathrm{~Hz}, 1 \mathrm{H}), 6.11$ (dd, $J=15.6,5.7 \mathrm{~Hz}, 1 \mathrm{H}), 5.13$ (d, $J=5.6 \mathrm{~Hz}, 1 \mathrm{H}), 0.95$ (s, 9H), 0.23 (s, 3H), 0.20 (s, 3H).

${ }^{13}$ C NMR (125 MHz, $\left.\mathrm{CDCl}_{3}\right) \delta 163.09\left(\mathrm{e}, \mathrm{d},{ }^{1} J_{C-F}=248.6 \mathrm{~Hz}\right), 132.53(\mathrm{o}), 131.43\left(\mathrm{e}, \mathrm{d},{ }^{4} J_{C-F}=3.8\right.$ $\mathrm{Hz}), 128.76\left(\mathrm{o}, \mathrm{d},{ }^{3} J_{C-F}=7.7 \mathrm{~Hz}\right), 123.63\left(\mathrm{o}, \mathrm{d},{ }^{5} J_{C-F}=1.9 \mathrm{~Hz}\right), 118.53(\mathrm{e}), 115.90\left(\mathrm{o}, \mathrm{d},{ }^{2} J_{C-F}=21.5\right.$ Hz), 62.67 (o), 25.68 (o), 18.32 (e), -4.83 (o), -4.90 (o). 
IR (Neat) 2955 (w), 2932 (w), 2859 (w), 1603 (w), 1510 (m), $1471(\mathrm{w}), 1256$ (m), 1230 (m), 1159 (m), 1096 (m), 968 (m), 835 (vs), 780 (s) $\mathrm{cm}^{-1}$.

HRMS (EI, M ${ }^{+}$) calcd for $\mathrm{C}_{16} \mathrm{H}_{22}$ FNOSi 291.1455, found 291.1461.

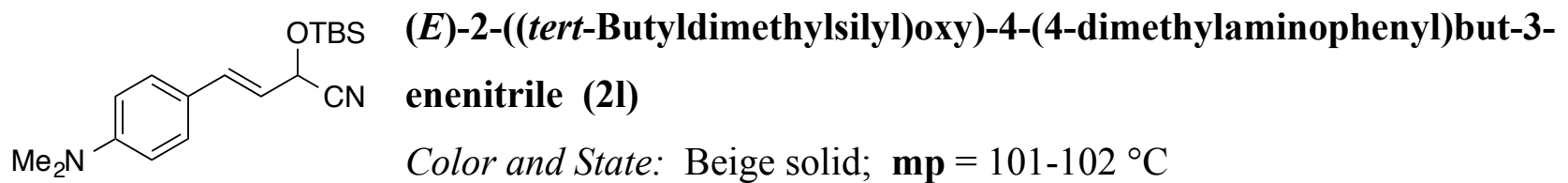

${ }^{1}$ H NMR $\left(500 \mathrm{MHz}, \mathrm{CDCl}_{3}\right) \delta 7.30(\mathrm{~d}, J=8.6 \mathrm{~Hz}, 2 \mathrm{H}), 6.69(\mathrm{~d}, J=16.2 \mathrm{~Hz}, 1 \mathrm{H}), 6.68(\mathrm{~d}, J=8.5$ $\mathrm{Hz}, 2 \mathrm{H}), 5.98(\mathrm{dd}, J=15.7,6.4 \mathrm{~Hz}, 1 \mathrm{H}), 5.10$ (d, $J=6.3 \mathrm{~Hz}, 1 \mathrm{H}), 2.99(\mathrm{~s}, 6 \mathrm{H}), 0.95(\mathrm{~s}, 9 \mathrm{H}), 0.22(\mathrm{~s}$, $3 \mathrm{H}), 0.19(\mathrm{~s}, 3 \mathrm{H})$.

${ }^{13}$ C NMR (125 MHz, $\mathrm{CDCl}_{3}$ ) $\delta 150.89$ (e), 134.17 (o), 128.22 (o), 123.27 (e), 119.14 (o), 119.08 (e), 112.25 (o), 63.35 (o), 40.43 (o), 25.71 (o), 18.31 (e), -4.74 (o), -4.75 (o).

IR (Neat) 2956 (w), 2931 (m), 2898 (w), 2857 (m), 2239 (w), 1641 (m), 1609 (m), 1473 (m), 1254 (m), 1187 (m), 1095 (m), 1055 (vs), 977 (s), 841 (s), 780 (s) $\mathrm{cm}^{-1}$.

HRMS (EI, M ${ }^{+}$) calcd for $\mathrm{C}_{18} \mathrm{H}_{28} \mathrm{~N}_{2} \mathrm{OSi} 316.1971$, found 316.1965 .

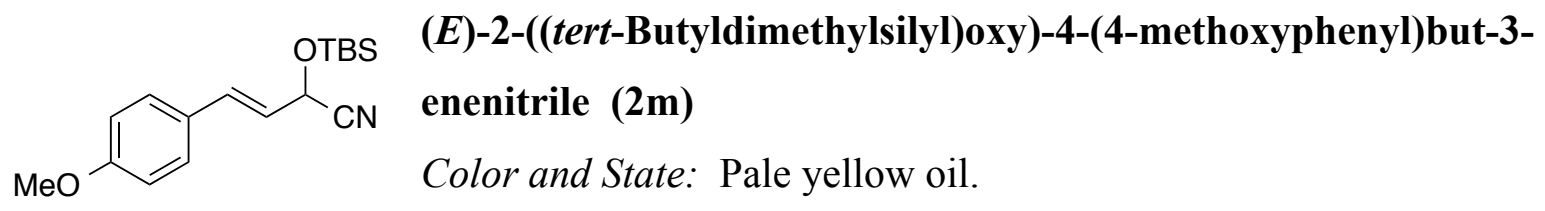

${ }^{1}$ H NMR $\left(500 \mathrm{MHz}, \mathrm{CDCl}_{3}\right) \delta$ 7.36-7.33 (m, 2H), 6.89-6.87 (m, 2H), $6.73(\mathrm{~d}, J=15.7 \mathrm{~Hz}, 1 \mathrm{H}), 6.05$ $(\mathrm{dd}, J=15.7,6.0 \mathrm{~Hz}, 1 \mathrm{H}), 5.11(\mathrm{dd}, J=6.0,1.2 \mathrm{~Hz}, 1 \mathrm{H}), 3.82(\mathrm{~s}, 3 \mathrm{H}), 0.93(\mathrm{~s}, 9 \mathrm{H}), 0.21(\mathrm{~s}, 3 \mathrm{H})$, $0.18(\mathrm{~s}, 3 \mathrm{H})$.

${ }^{13}$ C NMR (125 MHz, $\mathrm{CDCl}_{3}$ ) $\delta 160.22$ (e), 133.37 (o), 128.39 (o), 127.92 (e), 121.58 (o), 118.76 (e), 114.27 (o), 62.95 (o), 55.41 (o), 25.67 (o), 18.28 (e), -4.83 (o), -4.86 (o).

IR (Neat) 2957 (w), 2931 (w), 2859 (w), $2241(\mathrm{w}), 1652$ (w), 1606 (m), 1512 (m), 1472 (w), 1252 (s), 1176 (m), 1094 (s), 1072 (s), 979 (m), 833 (s), 778 (vs) $\mathrm{cm}^{-1}$.

HRMS (ESI $[\mathrm{M}+\mathrm{Na}]^{+}$) calcd for $\mathrm{C}_{17} \mathrm{H}_{25} \mathrm{NNaO}_{2} \mathrm{Si} 326.1552$, found 326.1554.

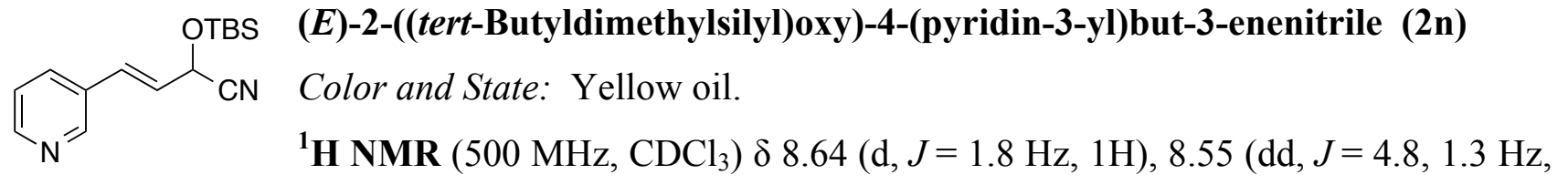
1H), $7.75(\mathrm{dt}, J=8.0,1.9 \mathrm{~Hz}, 1 \mathrm{H}), 7.31(\mathrm{dd}, J=7.9,4.9 \mathrm{~Hz}, 1 \mathrm{H}), 6.83(\mathrm{dd}, J=15.8,1.2 \mathrm{~Hz}, 1 \mathrm{H})$, 
$6.27(\mathrm{dd}, J=15.8,5.5 \mathrm{~Hz}, 1 \mathrm{H}), 5.16(\mathrm{dd}, J=5.3,1.6 \mathrm{~Hz}, 1 \mathrm{H}), 0.95$ (s, 9H), 0.24 (s, 3H), 0.20 (s, $3 \mathrm{H})$.

${ }^{13}$ C NMR (125 MHz, CDCl $) \delta 149.84$ (o), 148.81 (o), 133.53 (o), 130.95 (e), 130.05 (o), 126.08 (o), 123.70 (o), 118.19 (e), 62.40 (o), 25.65 (o), 18.30 (e), -4.88 (o), -4.97 (o).

IR (Neat) 2956 (w), 2931 (w), 2859 (w), 1735 (w), 1642 (w), 1473 (m), 1255 (m), 1184 (m), 1105 (m), 969 (m), 835 (s), 780 (vs) $\mathrm{cm}^{-1}$.

HRMS (EI, $\mathrm{M}^{+}$) calcd for $\mathrm{C}_{15} \mathrm{H}_{22} \mathrm{~N}_{2} \mathrm{O}_{2} \mathrm{Si}$ 274.1501, found 274.1509.

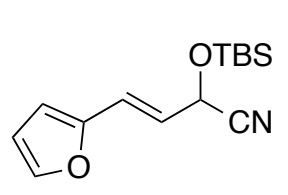

\section{(E)-2-((tert-Butyldimethylsilyl)oxy)-4-(furan-2-yl)but-3-enenitrile (2o)}

Color and State: Pale yellow oil.

${ }^{1}$ H NMR $\left(500 \mathrm{MHz}, \mathrm{CDCl}_{3}\right) \delta 7.39(\mathrm{~s}, 1 \mathrm{H}), 6.62(\mathrm{~d}, J=15.6 \mathrm{~Hz}, 1 \mathrm{H}), 6.41-6.36$ (m, 2H), 6.12 (dd, $J=15.6,5.5 \mathrm{~Hz}, 1 \mathrm{H}), 5.12$ (d, $J=5.5 \mathrm{~Hz}, 1 \mathrm{H}), 0.94$ (s, 9H), 0.21 (s, 3H), 0.18 (s, $3 \mathrm{H})$.

${ }^{13}$ C NMR (125 MHz, $\mathrm{CDCl}_{3}$ ) $\delta 151.01$ (e), 143.16 (o), 121.97 (o), 121.46 (o), 118.40 (e), 111.70 (o), 110.62 (o), 62.31 (o), 25.67 (o), 18.31 (e), -4.88 (o), -4.95 (o).

IR (Neat) 2956 (w), 2931 (m), 2859 (w), 1659 (w), 1472 (w), 1256 (m), 1111 (m), 1077 (m), 957 (m), 838 (vs), 780 (s) $\mathrm{cm}^{-1}$.

HRMS (ESI [M+Na] $]^{+}$calcd for $\mathrm{C}_{14} \mathrm{H}_{21} \mathrm{NNaO}_{2} \mathrm{Si} 286.1239$, found 286.1233 .

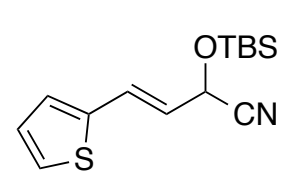

\section{(E)-2-((tert-Butyldimethylsilyl)oxy)-4-(thiophen-2-yl)but-3-enenitrile (2p)}

Color and State: Colorless oil.

${ }^{1}$ H NMR $\left(500 \mathrm{MHz}, \mathrm{CDCl}_{3}\right) \delta 7.25(\mathrm{~d}, J=5.1 \mathrm{~Hz}, 1 \mathrm{H}), 7.06(\mathrm{~d}, J=3.4 \mathrm{~Hz}, 1 \mathrm{H})$, $7.00(\mathrm{dd}, J=5.0,3.6 \mathrm{~Hz}, 1 \mathrm{H}), 6.93(\mathrm{~d}, J=15.6 \mathrm{~Hz}, 1 \mathrm{H}), 6.01(\mathrm{dd}, J=15.5,5.7 \mathrm{~Hz}, 1 \mathrm{H}), 5.10(\mathrm{dd}, J$ $=5.7,1.4 \mathrm{~Hz}, 1 \mathrm{H}), 0.94(\mathrm{~s}, 9 \mathrm{H}), 0.22(\mathrm{~s}, 3 \mathrm{H}), 0.19(\mathrm{~s}, 3 \mathrm{H})$.

${ }^{13}$ C NMR (125 MHz, $\mathrm{CDCl}_{3}$ ) $\delta 140.01$ (e), 127.84 (o), 127.75 (o), 126.81 (o), 126.05 (o), 122.95 (o), 118.42 (e), 62.48 (o), 25.70 (o), 18.33 (e), -4.80 (o), -4.85 (o).

IR (Neat) 2955 (m), 2930 (m), 2858 (m), 1647 (w), 1472 (w), 1255 (m), 1103 (m), 1081 (m), 955 (m), $839(\mathrm{~s}), 782(\mathrm{~s}) \mathrm{cm}^{-1}$.

HRMS (ESI $[\mathrm{M}+\mathrm{Na}]^{+}$) calcd for $\mathrm{C}_{14} \mathrm{H}_{21} \mathrm{NNaOSSi} 302.1011$, found 302.1013 . 


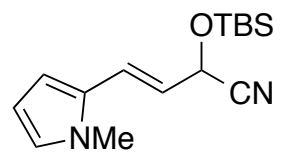
enenitrile (2q)

Color and State: Yellow oil.

${ }^{1}$ H NMR $\left(500 \mathrm{MHz}, \mathrm{CDCl}_{3}\right) \delta 6.69(\mathrm{~d}, J=15.6 \mathrm{~Hz}, 1 \mathrm{H}), 6.64(\mathrm{t}, J=1.9 \mathrm{~Hz}, 1 \mathrm{H}), 6.42(\mathrm{dd}, J=3.5$, $1.3 \mathrm{~Hz}, 1 \mathrm{H}), 6.12$ (t, $J=3.1 \mathrm{~Hz}, 1 \mathrm{H}), 5.93(\mathrm{dd}, J=15.6,5.9 \mathrm{~Hz}, 1 \mathrm{H}), 5.10$ (dd, $J=5.8,1.2 \mathrm{~Hz}, 1 \mathrm{H})$, $3.64(\mathrm{~s}, 3 \mathrm{H}), 0.94(\mathrm{~s}, 9 \mathrm{H}), 0.21(\mathrm{~s}, 3 \mathrm{H}), 0.18(\mathrm{~s}, 3 \mathrm{H})$.

${ }^{13}$ C NMR (125 MHz, $\mathrm{CDCl}_{3}$ ) $\delta 129.33$ (e), 124.48 (o), 122.26 (o), 120.09 (o), 118.69 (e), 108.44 (o), 108.42 (o), 62.85 (o), 34.19 (o), 25.61 (o), 18.22 (e), -4.86 (o), -4.91 (o).

IR (Neat) 2954 (w), 2931 (w), 2859 (w), 1702 (w), 1648 (w), 1473 (m), 1255 (m), 1103 (m), 1056 (m), $954(\mathrm{~m}), 838(\mathrm{~s}), 780(\mathrm{~s}) \mathrm{cm}^{-1}$.

HRMS (EI, $\mathrm{M}^{+}$) calcd for $\mathrm{C}_{15} \mathrm{H}_{24} \mathrm{~N}_{2} \mathrm{OSi} 276.1658$, found 276.1651 .

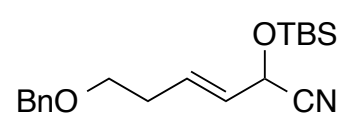

(E)-6-(benzyloxy)-2-(tert-Butyldimethylsilyloxy)hex-3-enenitrile (2r)

Color and State: Colorless oil.

${ }^{1} \mathbf{H}$ NMR $\left(500 \mathrm{MHz}, \mathrm{CDCl}_{3}\right) \delta$ 7.37-7.32 (m, 4H), 7.30-7.27 (m, 1H), 5.99

$(\mathrm{dtd}, J=15.2,6.9,1.3 \mathrm{~Hz}, 1 \mathrm{H}), 5.61(\mathrm{ddt}, J=15.4,5.8,1.4 \mathrm{~Hz}, 1 \mathrm{H}), 4.92(\mathrm{dd}, J=5.8,0.9 \mathrm{~Hz}, 1 \mathrm{H})$, $4.51(\mathrm{~s}, 2 \mathrm{H}), 3.54$ (t, $J=6.5 \mathrm{~Hz}, 2 \mathrm{H}), 2.42$ (app. q, $J=6.6 \mathrm{~Hz}, 2 \mathrm{H}), 0.91(\mathrm{~s}, 9 \mathrm{H}), 0.17$ (s, 3H), 0.14 $(\mathrm{s}, 3 \mathrm{H})$.

${ }^{13}$ C NMR (125 MHz, $\mathrm{CDCl}_{3}$ ) $\delta 138.32$ (e), 132.39 (o), 128.52 (o), 127.77 (o), 126.88 (o), 118.82 (e), 73.13 (e), 69.03 (e), 62.64 (o), 32.42 (e), 25.66 (o), 18.26 (e), -4.88 (o), -4.91 (o).

IR (Neat) 3032 (w), 2955 (w), 2931 (w), 2858 (m), 1671 (w), 1473 (w), 1363 (w), 1255 (m), 1098 (s), 1066 (s), 967 (m), 837 (vs), 780 (s) $\mathrm{cm}^{-1}$.

HRMS (ESI, $[\mathrm{M}+\mathrm{H}]^{+}$) calcd for $\mathrm{C}_{19} \mathrm{H}_{30} \mathrm{NO}_{2} \mathrm{Si} 332.2040$, found 332.2037.

\section{Representative Experimental Procedure for the Rhodium-Catalyzed Allylic Substitution with Alkenyl Cyanohydrin Pronucleophiles}

Lithium bis(trimethylsilyl)amide $(1.40 \mathrm{~mL}, 1.40 \mathrm{mmol}$; $1 \mathrm{M}$ solution in tetrahydrofuran) was added dropwise to a stirred solution of (E)-2-((tert-butyldimethylsilyl)oxy)-6-phenylhex-3-enenitrile $2 \mathbf{a}$ $(301.0 \mathrm{mg}, 1.0 \mathrm{mmol})$ in anhydrous tetrahydrofuran $(3 \mathrm{~mL})$ at $-10^{\circ} \mathrm{C}$ under an atmosphere of argon. The anion was allowed to form over $c a .30$ minutes, resulting in an orange homogeneous solution. In a separate flask, $[\mathrm{RhCl}(\mathrm{COD})]_{2}(6.20 \mathrm{mg}, 0.013 \mathrm{mmol})$ and triphenylphosphite $(13.1 \mu \mathrm{L}, 0.050$ mmol) were dissolved in anhydrous tetrahydrofuran $(2 \mathrm{~mL})$ at room temperature under an 
atmosphere of argon. The mixture was stirred for $c a .5$ minutes, resulting in a light yellow homogeneous solution, which was then cooled to $-10{ }^{\circ} \mathrm{C}$. The catalyst solution was then added to the anion via Teflon $^{\circledR}$ cannula, followed immediately by the addition of methyl (3-methyl-5phenylpent-1-en-3-yl) carbonate $1 \mathrm{a}(117.0 \mathrm{mg}, 0.50 \mathrm{mmol})$ via tared $500 \mu \mathrm{L}$ gastight syringe. The mixture was allowed to stir for $c a$. 16 hours and then cooled to $-40{ }^{\circ} \mathrm{C}$ (dry ice/acetonitrile bath). A $1 \mathrm{M}$ solution of TBAF in tetrahydrofuran $(3.00 \mathrm{~mL}, 3.00 \mathrm{mmol})$ was then added dropwise and the solution was allowed to stir for $c a .1$ hour at $-40{ }^{\circ} \mathrm{C}$. The reaction mixture was quenched with saturated aqueous ammonium chloride solution $(2 \mathrm{~mL})$, allowed to warm to room temperature and then partitioned between diethyl ether and saturated aqueous ammonium chloride solution. The combined organic layers were dried (anhyd. $\mathrm{MgSO}_{4}$ ), filtered and concentrated in vacuo to give the crude product. Purification by flash column chromatography (silica gel, eluting with 3-9\% diethyl ether/hexane) afforded the ketone $\mathbf{3 a}(132.2 \mathrm{mg}, 83 \%)$ as a pale yellow oil.

\section{Spectral Data for the $\alpha, \beta$-Unsaturated Ketone Products 3a-x}

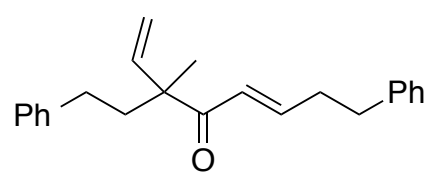

\section{(E)-3-Methyl-3-phenethyl-8-phenylocta-1,5-dien-4-one (3a)}

Color and State: Pale yellow oil; $b: l \geq 19: 1$

${ }^{1} \mathbf{H}$ NMR $\left(500 \mathrm{MHz}, \mathrm{CDCl}_{3}\right) \delta$ 7.29-7.25 (m, 4H), 7.19-7.13 (m, 6H), $6.98(\mathrm{dt}, J=15.2,7.0 \mathrm{~Hz}, 1 \mathrm{H}), 6.42(\mathrm{~d}, J=15.3 \mathrm{~Hz}, 1 \mathrm{H}), 5.91(\mathrm{dd}, J=$ 17.5, $10.8 \mathrm{~Hz}, 1 \mathrm{H}), 5.23(\mathrm{~d}, J=10.8 \mathrm{~Hz}, 1 \mathrm{H}), 5.15$ (d, $J=17.5 \mathrm{~Hz}, 1 \mathrm{H}), 2.77$ (t, $J=7.7 \mathrm{~Hz}, 2 \mathrm{H})$, 2.54-2.48 (m, 3H), $2.40\left(\mathrm{dt}, \mathrm{B}\right.$ of $\left.\mathrm{ABX}_{2}, J_{A B}=13.0 \mathrm{~Hz}, J_{B X}=5.0 \mathrm{~Hz}, 1 \mathrm{H}\right), 1.95\left(\mathrm{dt}, \mathrm{A}\right.$ of $\mathrm{ABX}_{2}, J_{A B}=$ $\left.13.1 \mathrm{~Hz}, J_{A X}=5.0 \mathrm{~Hz}, 1 \mathrm{H}\right), 1.87\left(\mathrm{dt}, \mathrm{B}\right.$ of $\left.\mathrm{ABX}_{2}, J_{A B}=13.1 \mathrm{~Hz}, J_{B X}=5.0 \mathrm{~Hz}, 1 \mathrm{H}\right), 1.26(\mathrm{~s}, 3 \mathrm{H})$.

${ }^{13}$ C NMR (125 MHz, $\mathrm{CDCl}_{3}$ ) $\delta 200.69$ (e), 146.37 (o), 142.40 (e), 141.27 (o), 140.86 (e), 128.50 (o), 128.44 (o), 128.39 (o), 126.18 (o), 125.91 (o), 125.85 (o), 115.65 (e), 53.10 (e), 39.20 (e), 34.49 (e), 34.20 (e), 30.79 (e), 20.04 (o).

IR (Neat) 3085 (w), 3062 (w), 3026 (w), 2930 (w), 2860 (w), 1689 (s), 1623 (s), 1604 (m), 1497 (m), $1454(\mathrm{~m}), 1127(\mathrm{w}), 920(\mathrm{~m}), 698(\mathrm{~s}) \mathrm{cm}^{-1}$.

HRMS (ESI [M+Na $]^{+}$) calcd for $\mathrm{C}_{23} \mathrm{H}_{26} \mathrm{NaO} 341.1881$, found 341.1872.

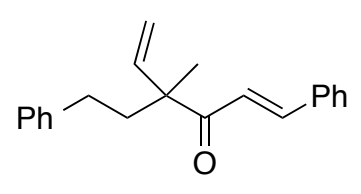

(E)-4-methyl-4-phenehyl-1-phenylhexa-1,5-dien-3-one (3b)

Color and State: Pale yellow oil; $b: l \geq 19: 1$

${ }^{1} \mathbf{H}$ NMR $\left(500 \mathrm{MHz}, \mathrm{CDCl}_{3}\right) \delta 7.69(\mathrm{~d}, J=15.7 \mathrm{~Hz}, 1 \mathrm{H}), 7.57-7.55(\mathrm{~m}, 2 \mathrm{H})$, 7.39-7.32 (m, 3H), 7.28-7.25 (m, 2H), 7.18-7.16 (m, 3H), $7.08(\mathrm{~d}, J=15.7 \mathrm{~Hz}, 1 \mathrm{H}), 6.03(\mathrm{dd}, J=$ 
$17.5,10.8 \mathrm{~Hz}, 1 \mathrm{H}), 5.30(\mathrm{~d}, J=10.8 \mathrm{~Hz}, 1 \mathrm{H}), 5.26(\mathrm{~d}, J=17.5 \mathrm{~Hz}, 1 \mathrm{H}), 2.59\left(\mathrm{dt}, \mathrm{A}\right.$ of $\mathrm{ABX}_{2}, J_{A B}=$ $\left.13.0 \mathrm{~Hz}, J_{A X}=4.9 \mathrm{~Hz}, 1 \mathrm{H}\right), 2.47\left(\mathrm{dt}, \mathrm{B}\right.$ of $\left.\mathrm{ABX}_{2}, J_{A B}=13.0 \mathrm{~Hz}, J_{B X}=5.0 \mathrm{~Hz}, 1 \mathrm{H}\right), 2.08(\mathrm{dt}, \mathrm{A}$ of $\left.\mathrm{ABX}_{2}, J_{A B}=13.1 \mathrm{~Hz}, J_{A X}=5.0 \mathrm{~Hz}, 1 \mathrm{H}\right), 1.98\left(\mathrm{dt}, \mathrm{B}\right.$ of $\left.\mathrm{ABX}_{2}, J_{A B}=13.1 \mathrm{~Hz}, J_{B X}=4.9 \mathrm{~Hz}, 1 \mathrm{H}\right), 1.37$ (s, 3H).

${ }^{13}$ C NMR (125 MHz, CDCl $) \delta 200.85$ (e), 143.03 (o), 142.43 (e), 141.41 (o), 134.92 (e), 130.45 (o), 128.98 (o), 128.52 (o), 128.49 (o), 128.46 (o), 125.98 (o), 121.83 (o), 115.98 (e), 53.55 (e), 39.37 (e), 30.93 (e), 20.31 (o).

IR (Neat) 3083 (w), 3061 (w), 3026 (w), 2974 (w), 2931 (w), 2863 (w), 1683 (s), 1631 (w), 1607 (s), $1496(\mathrm{~m}), 1449(\mathrm{~m}), 1202(\mathrm{w}), 1126(\mathrm{w}), 921(\mathrm{~m}), 699(\mathrm{~m}) \mathrm{cm}^{-1}$.

HRMS (ESI $[\mathrm{M}+\mathrm{Na}]^{+}$) calcd for $\mathrm{C}_{21} \mathrm{H}_{22} \mathrm{NaO} 313.1568$, found 313.1577 .

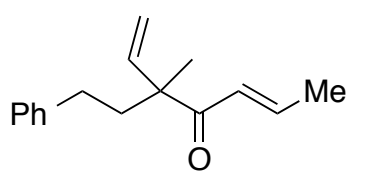

\section{(E)-3-Methyl-3-phenethylhepta-1,5-dien-4-one (3c)}

Color and State: Pale yellow oil; $b: l \geq 19: 1$

${ }^{1}$ H NMR $\left(500 \mathrm{MHz}, \mathrm{CDCl}_{3}\right) \delta$ 7.29-7.26 (m, 2H), 7.19-7.16 (m, 3H), 6.97 (dq, $J=15.1,7.0 \mathrm{~Hz}, 1 \mathrm{H}), 6.48(\mathrm{dq}, J=15.2,1.6 \mathrm{~Hz}, 1 \mathrm{H}), 5.96$ (dd, $J=17.5,10.8 \mathrm{~Hz}, 1 \mathrm{H}), 5.25$ (dd, $J=10.8,0.7 \mathrm{~Hz}, 1 \mathrm{H}), 5.19(\mathrm{dd}, J=17.5,0.7 \mathrm{~Hz}, 1 \mathrm{H}), 2.54\left(\mathrm{dt}, \mathrm{A}\right.$ of $\mathrm{ABX}_{2}, J_{A B}=13.0 \mathrm{~Hz}, J_{A X}=5.0$ $\mathrm{Hz}, 1 \mathrm{H}$ ), $2.43\left(\mathrm{dt}, \mathrm{B}\right.$ of $\mathrm{ABX} 2, J_{A B}=13.0 \mathrm{~Hz}, J_{B X}=5.0 \mathrm{~Hz}, 1 \mathrm{H}$ ), 2.00 (ddd, A of ABXY, $J_{\mathrm{AB}}=13.6$ $\left.\mathrm{Hz}, J_{\mathrm{AX}}=12.5 \mathrm{~Hz}, J_{\mathrm{AY}}=5.0 \mathrm{~Hz}, 1 \mathrm{H}\right), 1.93-1.87(\mathrm{~m}, 1 \mathrm{H}), 1.88(\mathrm{dd}, J=6.9,1.7 \mathrm{~Hz}, 3 \mathrm{H}), 1.30(\mathrm{~s}, 3 \mathrm{H})$. ${ }^{13}$ C NMR (125 MHz, CDCl $) \delta 200.75$ (e), 143.06 (o), 142.50 (e), 141.43 (o), 128.50 (o), 128.43 (o), 126.88 (o), 125.95 (o), 115.64 (e), 53.07 (e), 39.25 (e), 30.85 (e), 20.08 (o), 18.36 (o).

IR (Neat) 3086 (w), 3027 (w), 2974 (w), 2936 (w), 2863 (w), 1690 (s), 1625 (s), 1497 (w), 1455 (m), $1289(\mathrm{~m}), 1133(\mathrm{w}), 919(\mathrm{~s}), 698(\mathrm{~s}) \mathrm{cm}^{-1}$.

HRMS (ESI $[\mathrm{M}+\mathrm{H}]^{+}$) calcd for $\mathrm{C}_{16} \mathrm{H}_{21} \mathrm{O} 229.1587$, found 229.1584 .

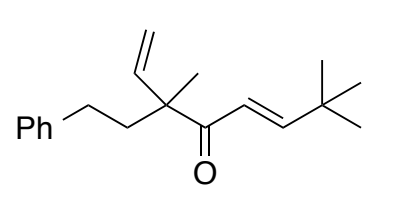

\section{(E)-3,7,7-Trimethyl-3-phenethylocta-1,5-dien-4-one (3d)}

Color and State: Pale yellow oil; $b: l \geq 19: 1$

${ }^{1}$ H NMR $\left(500 \mathrm{MHz}, \mathrm{CDCl}_{3}\right) \delta$ 7.29-7.26 (m, 2H), 7.19-7.15 (m, 3H), $6.96(\mathrm{~d}$, $J=15.6 \mathrm{~Hz}, 1 \mathrm{H}), 6.36$ (d, $J=15.6 \mathrm{~Hz}, 1 \mathrm{H}), 5.97$ (dd, $J=17.5,10.8 \mathrm{~Hz}, 1 \mathrm{H}), 5.25$ (d, $J=10.8 \mathrm{~Hz}$, $1 \mathrm{H}), 5.19(\mathrm{~d}, J=17.6 \mathrm{~Hz}, 1 \mathrm{H}), 2.54\left(\mathrm{dt}, \mathrm{A}\right.$ of $\left.\mathrm{ABX}_{2}, J_{A B}=13.0 \mathrm{~Hz}, J_{A X}=5.0 \mathrm{~Hz}, 1 \mathrm{H}\right), 2.43$ (dt, B of $\left.\mathrm{ABX}_{2}, J_{A B}=13.0 \mathrm{~Hz}, J_{B X}=4.9 \mathrm{~Hz}, 1 \mathrm{H}\right), 2.00\left(\mathrm{dt}, \mathrm{A}\right.$ of $\left.\mathrm{ABX}_{2}, J_{A B}=13.1 \mathrm{~Hz}, J_{A X}=4.9 \mathrm{~Hz}, 1 \mathrm{H}\right), 1.91$ (dt, B of $\left.\mathrm{ABX}_{2}, J_{A B}=13.1 \mathrm{~Hz}, J_{B X}=5.0 \mathrm{~Hz}, 1 \mathrm{H}\right), 1.30(\mathrm{~s}, 3 \mathrm{H}), 1.07(\mathrm{~s}, 9 \mathrm{H})$. 
${ }^{13}$ C NMR (125 MHz, $\mathrm{CDCl}_{3}$ ) $\delta 201.39$ (e), 157.48 (o), 142.50 (e), 141.52 (o), 128.47 (o), 128.39 (o), 125.91 (o), 120.23 (o), 115.52 (e), 53.39 (e), 39.46 (e), 33.91 (e), 30.90 (e), 28.86 (o), 20.23 (o).

IR (Neat) 3086 (w), 3063 (w), 3027 (w), 2961 (m), 2867 (w), 1690 (s), 1621 (s), 1497 (w), 1456 (m), $1126(\mathrm{w}), 919(\mathrm{~m}), 699(\mathrm{~s}) \mathrm{cm}^{-1}$.

HRMS (ESI [M+Na] $]^{+}$) calcd for $\mathrm{C}_{19} \mathrm{H}_{26} \mathrm{NaO} 293.1881$, found 293.1886.

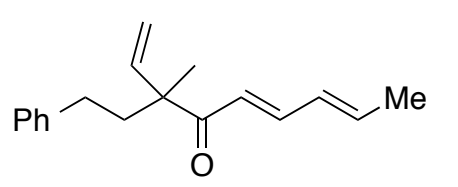

\section{(5E,7E)-3,Methyl-3-phenethylnona-1,5,7-trien-4-one (3e)}

Color and State: Yellow oil; $b: l \geq 19: 1$

The product was isolated as a $6: 1$ mixture of $(5 E, 7 E)$ and $(5 E, 7 Z)$ isomers. Data is provided for the major isomer only.

${ }^{1}$ H NMR $\left(500 \mathrm{MHz}, \mathrm{CDCl}_{3}\right) \delta$ 7.30-7.26 (m, 3H), 7.19-7.16 (m, 3H), $6.41(\mathrm{~d}, J=14.7 \mathrm{~Hz}, 1 \mathrm{H})$, 6.25-6.13 (m, 2H), $5.97(\mathrm{dd}, J=17.5,10.8 \mathrm{~Hz}, 1 \mathrm{H}), 5.24(\mathrm{dd}, J=10.7,0.8 \mathrm{~Hz}, 1 \mathrm{H}), 5.19$ (dd, $J=$ 17.5, 0.7 Hz, 1H), $2.54\left(\mathrm{dt}, \mathrm{A}\right.$ of $\left.\mathrm{ABX}_{2}, J_{A B}=13.0 \mathrm{~Hz}, J_{A X}=5.0 \mathrm{~Hz}, 1 \mathrm{H}\right), 2.44\left(\mathrm{dt}, \mathrm{B}\right.$ of $\mathrm{ABX}_{2}, J_{A B}=$ $\left.13.0 \mathrm{~Hz}, J_{B X}=5.0 \mathrm{~Hz}, 1 \mathrm{H}\right), 2.00\left(\mathrm{dt}, \mathrm{A}\right.$ of $\left.\mathrm{ABX}_{2}, J_{A B}=13.1 \mathrm{~Hz}, J_{A X}=5.0 \mathrm{~Hz}, 1 \mathrm{H}\right), 1.91(\mathrm{dt}, \mathrm{B}$ of $\left.\mathrm{ABX}_{2}, J_{A B}=13.2 \mathrm{~Hz}, J_{B X}=4.7 \mathrm{~Hz}, 1 \mathrm{H}\right), 1.86(\mathrm{~d}, J=5.0 \mathrm{~Hz}, 3 \mathrm{H}), 1.30(\mathrm{~s}, 3 \mathrm{H})$.

${ }^{13}$ C NMR (125 MHz, $\mathrm{CDCl}_{3}$ ) $\delta 201.32$ (e), 143.40 (o), 142.49 (e), 141.53 (o), 140.66 (o), 130.41 (o), 128.46 (o), 128.40 (o), 125.90 (o), 123.08 (o), 115.51 (e), 53.21 (e), 39.36 (e), 30.86 (e), 20.19 (o), $18.91(\mathrm{o})$.

IR (Neat) 3085 (w), 3026 (w), 2973 (w), 2933 (w), 2863 (w), 1682 (s), 1633 (m), 1591 (s), 1497 (w), $1455(\mathrm{w}), 1329$ (m), 1124 (w), 1031 (m), 1000 (s), 919 (m), 699 (s) cm ${ }^{-1}$.

HRMS (ESI [M+Na] $]^{+}$) calcd for $\mathrm{C}_{18} \mathrm{H}_{22} \mathrm{NaO} 277.1568$, found 277.1579.

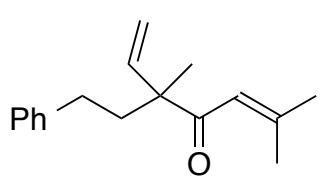

\section{3,6-Dimethyl-3-phenethylhepta-1,5-dien-4-one (3f)}

Color and State: Pale yellow oil; $b: l \geq 19: 1$

${ }^{1} \mathbf{H}$ NMR $\left(500 \mathrm{MHz}, \mathrm{CDCl}_{3}\right) \delta$ 7.29-7.27 (m, 2H), 7.19-7.16 (m, 3H), 6.28-6.27 (m, 1H), $5.98(\mathrm{dd}, J=17.5,10.8 \mathrm{~Hz}, 1 \mathrm{H}), 5.21(\mathrm{dd}, J=10.7,0.9 \mathrm{~Hz}, 1 \mathrm{H}), 5.17$ (dd, $J=17.5,0.8 \mathrm{~Hz}$, $1 \mathrm{H}), 2.54\left(\mathrm{dt}, \mathrm{A}\right.$ of $\left.\mathrm{ABX}_{2}, J_{A B}=13.0 \mathrm{~Hz}, J_{A X}=4.9 \mathrm{~Hz}, 1 \mathrm{H}\right), 2.43\left(\mathrm{dt}, \mathrm{B}\right.$ of $\mathrm{ABX}_{2}, J_{A B}=12.8 \mathrm{~Hz}, J_{B X}$ $=4.9 \mathrm{~Hz}, 1 \mathrm{H}), 2.14(\mathrm{~d}, J=1.1 \mathrm{~Hz}, 3 \mathrm{H}), 1.99\left(\mathrm{dt}, \mathrm{A}\right.$ of $\left.\mathrm{ABX}_{2}, J_{A B}=13.1 \mathrm{~Hz}, J_{A X}=4.9 \mathrm{~Hz}, 1 \mathrm{H}\right), 1.90$ $(\mathrm{d}, J=1.1 \mathrm{~Hz}, 3 \mathrm{H}), 1.89\left(\mathrm{dt}, \mathrm{B}\right.$ of $\left.\mathrm{ABX}_{2}, J_{A B}=13.1 \mathrm{~Hz}, J_{B X}=5.0 \mathrm{~Hz}, 1 \mathrm{H}\right), 1.28(\mathrm{~s}, 3 \mathrm{H})$.

${ }^{13}$ C NMR (125 MHz, $\mathrm{CDCl}_{3}$ ) $\delta 202.45$ (e), 156.04 (e), 142.64 (e), 142.12 (o), 128.47 (o), 128.41 (o), 125.88 (o), 120.88 (o), 114.91 (e), 53.85 (e), 39.68 (e), 30.95 (e), 28.02 (o), 21.04 (o), 20.27 (o). 
IR (Neat) 3087 (w), 3064 (w), 3028 (w), 2931 (w), 2861 (w), 1681 (m), 1618 (s), 1497 (w), 1453 (m), 1126 (w), 1094 (w), 1023 (m), 917 (m), 699 (vs) $\mathrm{cm}^{-1}$.

HRMS (ESI $[\mathrm{M}+\mathrm{H}]^{+}$) calcd for $\mathrm{C}_{17} \mathrm{H}_{23} \mathrm{O} 243.1743$, found 243.1743.

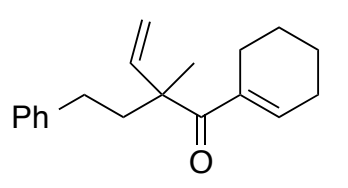

\section{1-(Cyclohex-1-en-1-yl)-2-methyl-2-phenethylbut-3-en-1-one (3g)}

Color and State: Pale yellow oil; $b: l \geq 19: 1$

${ }^{1} \mathbf{H}$ NMR $\left(500 \mathrm{MHz}, \mathrm{CDCl}_{3}\right) \delta$ 7.28-7.25 (m, 2H), 7.19-7.15 (m, 3H), 6.83-6.81 $(\mathrm{m}, 1 \mathrm{H}), 6.11(\mathrm{dd}, J=17.5,10.9 \mathrm{~Hz}, 1 \mathrm{H}), 5.15(\mathrm{~d}, J=10.7 \mathrm{~Hz}, 1 \mathrm{H}), 5.14(\mathrm{~d}, J=17.9 \mathrm{~Hz}, 1 \mathrm{H}), 2.54$ $\left(\mathrm{dt}, \mathrm{A}\right.$ of $\left.\mathrm{ABX}_{2}, J_{A B}=12.9 \mathrm{~Hz}, J_{A X}=4.8 \mathrm{~Hz}, 1 \mathrm{H}\right), 2.36\left(\mathrm{dt}, \mathrm{B}\right.$ of $\mathrm{ABX}_{2}, J_{A B}=13.0 \mathrm{~Hz}, J_{B X}=4.7 \mathrm{~Hz}$, $1 \mathrm{H}), 2.29-2.24(\mathrm{~m}, 1 \mathrm{H}), 2.20-2.14(\mathrm{~m}, 3 \mathrm{H}), 2.09\left(\mathrm{dt}, \mathrm{A}\right.$ of $\left.\mathrm{ABX}_{2}, J_{A B}=13.0 \mathrm{~Hz}, J_{A X}=4.7 \mathrm{~Hz}, 1 \mathrm{H}\right)$, $1.97\left(\mathrm{dt}, \mathrm{B}\right.$ of $\left.\mathrm{ABX}_{2}, J_{A B}=13.1 \mathrm{~Hz}, J_{B X}=4.9 \mathrm{~Hz}, 1 \mathrm{H}\right), 1.66-1.57(\mathrm{~m}, 4 \mathrm{H}), 1.34(\mathrm{~s}, 3 \mathrm{H})$.

${ }^{13}$ C NMR (125 MHz, $\mathrm{CDCl}_{3}$ ) $\delta 205.25$ (e), 143.75 (o), 142.68 (e), 138.41 (o), 137.76 (e), 128.47 (o), 128.43 (o), 125.88 (o), 113.45 (e), 53.28 (e), 41.73 (e), 31.05 (e), 25.69 (e), 25.08 (e), 23.47 (o), 22.32 (e), $21.63(\mathrm{e})$

IR (Neat) 3084 (w), 3062 (w), 3026 (w), 2932 (m), 2860 (w), 1659 (s), 1630 (m), 1604 (w), 1497 (w), $1454(\mathrm{~m}), 1031(\mathrm{w}), 912(\mathrm{~m}), 698(\mathrm{~s}) \mathrm{cm}^{-1}$.

HRMS (ESI $[\mathrm{M}+\mathrm{Na}]^{+}$) calcd for $\mathrm{C}_{19} \mathrm{H}_{24} \mathrm{NaO} 291.1725$, found 291.1735.

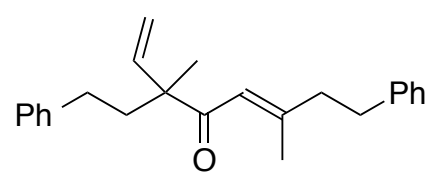

\section{(E)-3,6-Dimethyl-3-phenethyl-8-phenylocta-1,5-dien-4-one (3h)}

Color and State: Pale yellow oil; $b: l \geq 19: 1$

${ }^{1} \mathbf{H}$ NMR $\left(500 \mathrm{MHz}, \mathrm{CDCl}_{3}\right) \delta$ 7.29-7.25 (m, 4H), 7.19-7.14 (m, 6H), 6.20-6.19 (m, 1H), $5.90(\mathrm{dd}, J=17.6,10.6 \mathrm{~Hz}, 1 \mathrm{H}), 5.17(\mathrm{dd}, J=10.7,1.0 \mathrm{~Hz}, 1 \mathrm{H}), 5.11(\mathrm{dd}, J=$ 17.6, $1.0 \mathrm{~Hz}, 1 \mathrm{H}), 2.79(\mathrm{t}, J=7.8 \mathrm{~Hz}, 2 \mathrm{H}), 2.51\left(\mathrm{dt}, \mathrm{A}\right.$ of $\left.\mathrm{ABX}_{2}, J_{A B}=13.0 \mathrm{~Hz}, J_{A X}=5.1 \mathrm{~Hz}, 1 \mathrm{H}\right)$, $2.45(\mathrm{t}, J=7.8 \mathrm{~Hz}, 2 \mathrm{H}), 2.40\left(\mathrm{dt}, \mathrm{B}\right.$ of $\left.\mathrm{ABX}_{2}, J_{A B}=13.0 \mathrm{~Hz}, J_{B X}=5.1 \mathrm{~Hz}, 1 \mathrm{H}\right), 2.17(\mathrm{~d}, J=1.1 \mathrm{~Hz}$, $3 \mathrm{H}), 1.93,\left(\mathrm{dt}, \mathrm{A}\right.$ of $\left.\mathrm{ABX}_{2}, J_{A B}=13.0 \mathrm{~Hz}, J_{A X}=5.0 \mathrm{~Hz}, 1 \mathrm{H}\right), 1.83\left(\mathrm{dt}, \mathrm{B}\right.$ of $\mathrm{ABX}_{2}, J_{A B}=13.1 \mathrm{~Hz}, J_{B X}$ $=5.1 \mathrm{~Hz}, 1 \mathrm{H}), 1.22(\mathrm{~s}, 3 \mathrm{H})$.

${ }^{13}$ C NMR (125 MHz, $\mathrm{CDCl}_{3}$ ) $\delta 202.62$ (e), 157.90 (e), 142.61 (e), 141.98 (o), 141.05 (e), 128.53 (o), 128.46 (o), 128.43 (o), 126.19 (o), 125.89 (o), 120.98 (o), 114.92 (e), 53.93 (e), 43.12 (e), 39.63 (e), 33.98 (e), 30.92 (e), 20.22 (o), 19.72 (o).

IR (Neat) 3085 (w), 3063 (w), 3026 (w), 2930 (w), 2861 (w), 1680 (m), 1613 (m), 1496 (m), 1454 (m), $1124(\mathrm{w}), 1085(\mathrm{w}), 917(\mathrm{~m}), 697(\mathrm{vs}) \mathrm{cm}^{-1}$.

HRMS (EI, $\mathrm{M}^{+}$) calcd for $\mathrm{C}_{24} \mathrm{H}_{28} \mathrm{O} 332.2140$, found 332.2148. 


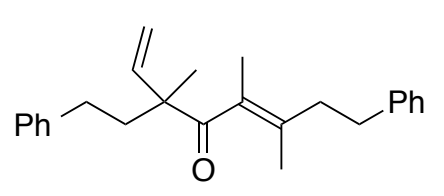

\section{(E)-3,5,6-Trimethyl-3-phenethyl-8-phenylocta-1,5-dien-4-one (3i)}

Color and State: Pale yellow oil; $b: l \geq 19: 1$

${ }^{1} \mathbf{H}$ NMR $\left(500 \mathrm{MHz}, \mathrm{CDCl}_{3}\right) \delta$ 7.30-7.26 (m, 4H), 7.21-7.16 (m, 6H), $5.94(\mathrm{dd}, J=17.5,10.7 \mathrm{~Hz}, 1 \mathrm{H}), 5.17(\mathrm{dd}, J=10.7,0.7 \mathrm{~Hz}, 1 \mathrm{H}), 5.13(\mathrm{dd}, J=17.5,0.7 \mathrm{~Hz}, 1 \mathrm{H})$, $2.72(\mathrm{t}, J=7.9 \mathrm{~Hz}, 2 \mathrm{H}), 2.50(\mathrm{dd}, J=9.3,8.1 \mathrm{~Hz}, 2 \mathrm{H}), 2.37-2.33(\mathrm{~m}, 2 \mathrm{H}), 1.97-1.88(\mathrm{~m}, 2 \mathrm{H}), 1.66$ (q, $J=1.4 \mathrm{~Hz}, 3 \mathrm{H}), 1.62(\mathrm{q}, J=1.4 \mathrm{~Hz}, 3 \mathrm{H}), 1.30(\mathrm{~s}, 3 \mathrm{H})$.

${ }^{13}$ C NMR (125 MHz, $\left.\mathrm{CDCl}_{3}\right) \delta 214.50$ (e), 142.43 (e), 141.96 (o), 141.80 (e), 133.11 (e), 132.67 (e), 128.52 (o), 128.50 (o), 128.47 (o), 126.12 (o), 125.95 (o), 114.62 (e), 54.06 (e), 40.78 (e), 35.34 (e), 33.70 (e), 30.77 (e), 20.45 (o), 19.62 (o), 16.22 (o).

IR (Neat) 3085 (w), 3062 (w), 3026 (w), 2929 (w), 2862 (w), 1745 (w), 1685 (s), 1636 (w), 1604 (w), 1497 (m), $1454(\mathrm{~m}), 1093(\mathrm{w}), 917$ (w), 699 (vs) $\mathrm{cm}^{-1}$.

HRMS (EI, $\mathrm{M}^{+}$) calcd for $\mathrm{C}_{25} \mathrm{H}_{30} \mathrm{O} 346.2297$, found 346.2291.

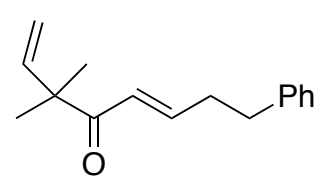

\section{(E)-3,3-Dimethyl-8-phenylocta-1,5-dien-4-one (3j)}

Color and State: Pale yellow oil; $b: l \geq 19: 1$

${ }^{1}$ H NMR $\left(500 \mathrm{MHz}, \mathrm{CDCl}_{3}\right) \delta$ 7.29-7.26 (m, 2H), 7.21-7.15 (m, 3H), $6.94(\mathrm{dt}, J$ $=15.3,7.0 \mathrm{~Hz}, 1 \mathrm{H}), 6.38(\mathrm{dt}, J=15.3,1.5 \mathrm{~Hz}, 1 \mathrm{H}), 5.87(\mathrm{dd}, J=17.3,10.8 \mathrm{~Hz}, 1 \mathrm{H}), 5.14(\mathrm{~d}, J=$ $10.6 \mathrm{~Hz}, 1 \mathrm{H}), 5.13$ (d, $J=17.8 \mathrm{~Hz}, 1 \mathrm{H}), 2.76$ (t, $J=7.7 \mathrm{~Hz}, 2 \mathrm{H}), 2.53-2.48(\mathrm{~m}, 2 \mathrm{H}), 1.20$ (s, 6H).

${ }^{13}$ C NMR (125 MHz, $\mathrm{CDCl}_{3}$ ) $\delta 201.35$ (e), 146.12 (o), 142.50 (o), 140.96 (e), 128.51 (o), 128.48 (o), 126.18 (o), 125.75 (o), 114.59 (e), 49.66 (e), 34.53 (e), 34.23 (e), 23.43 (o).

IR (Neat) 3086 (w), 3062 (w), 3027 (w), 2972 (w), 2931 (w), 1691 (s), 1624 (s), 1497 (w), 1455 (m), $1165(\mathrm{w}), 919(\mathrm{~m}), 699(\mathrm{~s}) \mathrm{cm}^{-1}$.

HRMS (ESI $[\mathrm{M}+\mathrm{H}]^{+}$) calcd for $\mathrm{C}_{16} \mathrm{H}_{21} \mathrm{O} 229.1587$, found 229.1586.

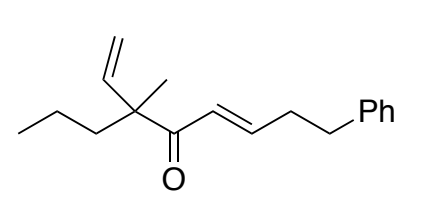

\section{(E)-6-Methyl-1-phenyl-6-vinylnon-3-en-5-one (3k)}

Color and State: Pale yellow oil; $b: l \geq 19: 1$

${ }^{1} \mathbf{H}$ NMR $\left(500 \mathrm{MHz}, \mathrm{CDCl}_{3}\right) \delta$ 7.29-7.26 (m, 2H), 7.20-7.15 (m, 3H), 6.93 (dt, $J=15.3,7.0 \mathrm{~Hz}, 1 \mathrm{H}), 6.37$ (dt, $J=15.3,1.5 \mathrm{~Hz}, 1 \mathrm{H}), 5.86(\mathrm{dd}, J=17.5,10.8 \mathrm{~Hz}, 1 \mathrm{H}), 5.15$ (dd, $J=10.7,0.9 \mathrm{~Hz}, 1 \mathrm{H}), 5.09(\mathrm{dd}, J=17.5,0.8 \mathrm{~Hz}, 1 \mathrm{H}), 2.76(\mathrm{t}, J=7.7 \mathrm{~Hz}, 2 \mathrm{H}), 2.53-2.48(\mathrm{~m}, 2 \mathrm{H})$, 1.62 (ddd, A of ABXY, $\left.J_{\mathrm{AB}}=13.6 \mathrm{~Hz}, J_{\mathrm{AX}}=12.3 \mathrm{~Hz}, J_{\mathrm{AY}}=4.8 \mathrm{~Hz}, 1 \mathrm{H}\right), 1.52$ (ddd, B of ABXY, $\left.J_{\mathrm{AB}}=13.5 \mathrm{~Hz}, J_{\mathrm{BX}}=12.4 \mathrm{~Hz}, J_{\mathrm{BY}}=4.4 \mathrm{~Hz}, 1 \mathrm{H}\right), 1.27-1.17(\mathrm{~m}, 1 \mathrm{H}), 1.16(\mathrm{~s}, 3 \mathrm{H}), 1.14-1.03(\mathrm{~m}, 1 \mathrm{H})$, $0.87(\mathrm{t}, J=7.3 \mathrm{~Hz}, 3 \mathrm{H})$. 
${ }^{13}$ C NMR (125 MHz, $\mathrm{CDCl}_{3}$ ) $\delta 201.19$ (e), 145.89 (o), 141.84 (o), 140.95 (e), 128.50 (o), 128.48 (o), 126.17 (o), 126.03 (o), 115.08 (e), 53.17 (e), 39.48 (e), 34.52 (e), 34.18 (e), 19.98 (o), 17.57 (e), $14.83(\mathrm{o})$.

IR (Neat) 3086 (w), 3063 (w), 3028 (w), 2959 (m), 2932 (m), 2872 (w), 1690 (s), 1624 (s), 1497 (w), $1454(\mathrm{~m}), 1189(\mathrm{w}), 917(\mathrm{~m}), 698(\mathrm{~s}) \mathrm{cm}^{-1}$.

HRMS (ESI [M+Na] $]^{+}$) calcd for $\mathrm{C}_{18} \mathrm{H}_{24} \mathrm{NaO} 279.1725$, found 279.1717.

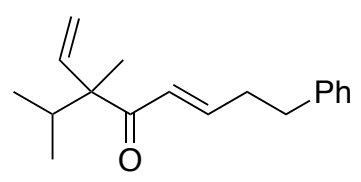

\section{(E)-3-Isopropyl-3-methyl-8-phenylocta-1,5-dien-4-one (3I)}

Color and State: Pale yellow oil; $b: l \geq 19: 1$

${ }^{1} \mathbf{H}$ NMR $\left(500 \mathrm{MHz}, \mathrm{CDCl}_{3}\right) \delta$ 7.30-7.26 (m, 2H), 7.20-7.15 (m, 3H), 6.93 (dt, $J=15.2,7.0 \mathrm{~Hz}, 1 \mathrm{H}), 6.41(\mathrm{dt}, J=15.2,1.5 \mathrm{~Hz}, 1 \mathrm{H}), 5.92(\mathrm{dd}, J=17.5,10.9 \mathrm{~Hz}, 1 \mathrm{H}), 5.20$ (dd, $J=10.9,0.9 \mathrm{~Hz}, 1 \mathrm{H}), 5.07(\mathrm{dd}, J=17.5,0.9 \mathrm{~Hz}, 1 \mathrm{H}), 2.77(\mathrm{t}, J=7.6 \mathrm{~Hz}, 2 \mathrm{H}), 2.53-2.49(\mathrm{~m}, 2 \mathrm{H})$, 2.13 (septet, $J=6.8 \mathrm{~Hz}, 1 \mathrm{H}), 1.08(\mathrm{~s}, 3 \mathrm{H}), 0.83(\mathrm{~d}, J=6.9 \mathrm{~Hz}, 3 \mathrm{H}), 0.74$ (d, $J=6.8 \mathrm{~Hz}, 3 \mathrm{H})$.

${ }^{13}$ C NMR (125 MHz, $\mathrm{CDCl}_{3}$ ) $\delta 201.28$ (e), 145.86 (o), 140.97 (e), 140.59 (o), 128.50 (o), 126.29 (o), 126.17 (o), 116.22 (e), 56.73 (e), 34.55 (e), 34.25 (e), 32.85 (o), 17.56 (o), 17.46 (o), 14.12 (o).

IR (Neat) 3085 (m), 3062 (w), 3027 (w), 2964 (m), 2937 (w), 1688 (s), 1623 (s), 1497 (w), 1454 (m), $1180(\mathrm{w}), 917(\mathrm{~m}), 699(\mathrm{~s}) \mathrm{cm}^{-1}$.

HRMS (ESI $[\mathrm{M}+\mathrm{Na}]^{+}$) calcd for $\mathrm{C}_{18} \mathrm{H}_{24} \mathrm{NaO} 279.1725$, found 279.1718.

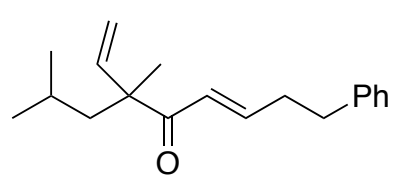

\section{(E)-6,8-Dimethyl-1-phenyl-6-vinylnon-3-en-5-one (3m)}

Color and State: Pale yellow oil; $b: l \geq 19: 1$

${ }^{1} \mathbf{H}$ NMR $\left(500 \mathrm{MHz}, \mathrm{CDCl}_{3}\right) \delta$ 7.29-7.26 (m, 2H), 7.20-7.15 (m, 3H), 6.92 (dt, $J=15.3,6.9 \mathrm{~Hz}, 1 \mathrm{H}), 6.40$ (dt, $J=15.3,1.5 \mathrm{~Hz}, 1 \mathrm{H}), 5.89$ (dd, $J=17.5,10.8 \mathrm{~Hz}, 1 \mathrm{H}), 5.14$ (dd, $J=10.8,0.8 \mathrm{~Hz}, 1 \mathrm{H}), 5.09(\mathrm{dd}, J=17.5,0.8 \mathrm{~Hz}, 1 \mathrm{H}), 2.76(\mathrm{t}, J=7.7 \mathrm{~Hz}, 2 \mathrm{H}), 2.54-2.49(\mathrm{~m}, 2 \mathrm{H})$, $1.65-1.52(\mathrm{~m}, 3 \mathrm{H}), 1.20$ (s, 3H), 0.86 (d, $J=6.3 \mathrm{~Hz}, 3 \mathrm{H}), 0.80$ (d, $J=6.2 \mathrm{~Hz}, 3 \mathrm{H})$.

${ }^{13}$ C NMR (125 MHz, $\mathrm{CDCl}_{3}$ ) $\delta 201.43$ (e), 145.74 (o), 142.41 (o), 140.98 (e), 128.53 (o), 128.49 (o), 126.30 (o), 126.18 (o), 114.89 (e), 53.20 (e), 46.15 (e), 34.54 (e), 34.18 (e), 24.88 (o), 24.74 (o), 24.22 (o), 20.34 (o).

IR (Neat) 3086 (w), 3063 (w), 3028 (w), 2955 (m), 2930 (m), 2869 (w), 1690 (s), 1624 (s), 1497 (w), $1455(\mathrm{~m}), 1170(\mathrm{w}), 916(\mathrm{~m}), 698(\mathrm{~s}) \mathrm{cm}^{-1}$.

HRMS (ESI [M+Na] $]^{+}$) calcd for $\mathrm{C}_{19} \mathrm{H}_{26} \mathrm{NaO} 293.1881$, found 293.1872. 


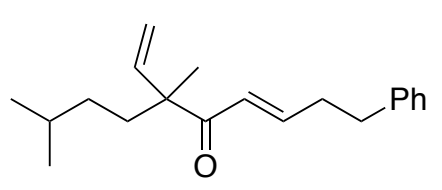

\section{(E)-6,9-Dimethyl-1-phenyl-6-vinyldec-3-en-5-one (3n)}

Color and State: Pale yellow oil; $b: l \geq 19: 1$

${ }^{1} \mathbf{H}$ NMR $\left(500 \mathrm{MHz}, \mathrm{CDCl}_{3}\right) \delta$ 7.29-7.26 (m, 2H), 7.20-7.15 (m, 3H), $6.93(\mathrm{dt}, J=15.3,7.0 \mathrm{~Hz}, 1 \mathrm{H}), 6.38(\mathrm{dt}, J=15.3,1.5 \mathrm{~Hz}, 1 \mathrm{H}), 5.86(\mathrm{dd}, J=17.5,10.8 \mathrm{~Hz}, 1 \mathrm{H}), 6.16$ (dd, $J=10.8,0.8 \mathrm{~Hz}, 1 \mathrm{H}), 5.09$ (dd, $J=17.6,0.8 \mathrm{~Hz}, 1 \mathrm{H}), 2.76(\mathrm{t}, J=7.7 \mathrm{~Hz}, 2 \mathrm{H}), 2.53-2.48(\mathrm{~m}$, $2 \mathrm{H}), 1.65\left(\mathrm{dt}, \mathrm{A}\right.$ of $\left.\mathrm{ABX}_{2}, J_{A B}=13.0 \mathrm{~Hz}, J_{A X}=4.7 \mathrm{~Hz}, 1 \mathrm{H}\right), 1.54\left(\mathrm{dt}, \mathrm{B}\right.$ of $\mathrm{ABX}_{2}, J_{A B}=13.1 \mathrm{~Hz}, J_{B X}$ $=4.4 \mathrm{~Hz}, 1 \mathrm{H}), 1.45$ (nonet, $J=6.7 \mathrm{~Hz}, 1 \mathrm{H}), 1.15(\mathrm{~s}, 3 \mathrm{H}), 1.07$ (ddt, A of $\mathrm{ABMX}_{2}, J_{A B}=12.8 \mathrm{~Hz}, J_{A M}$ $\left.=6.6 \mathrm{~Hz}, J_{A X}=4.5 \mathrm{~Hz}, 1 \mathrm{H}\right), 0.94\left(\mathrm{ddt}, \mathrm{B}\right.$ of $\left.\mathrm{ABMX}_{2}, J_{A B}=12.8 \mathrm{~Hz}, J_{B M}=6.8 \mathrm{~Hz}, J_{B X}=4.8 \mathrm{~Hz}, 1 \mathrm{H}\right)$, $0.85(\mathrm{~d}, J=6.6 \mathrm{~Hz}, 3 \mathrm{H}), 0.85(\mathrm{~d}, J=6.6 \mathrm{~Hz}, 3 \mathrm{H})$.

${ }^{13}$ C NMR (125 MHz, $\mathrm{CDCl}_{3}$ ) $\delta 201.31$ (e), 145.95 (o), 141.88 (o), 140.98 (e), 128.52 (o), 128.48 (o), 126.20 (o), 126.02 (o), 115.17 (e), 53.02 (e), 34.95 (e), 34.55 (e), 34.20 (e), 33.18 (e), 28.66 (o), 22.65 (o), 22.64 (o), 19.96 (o).

IR (Neat) 3086 (w), 3063 (w), 3028 (w), 2954 (m), 2932 (m), 2869 (m), 1691 (s), 1624 (vs), 1497 (w), $1455(\mathrm{~m}), 1170(\mathrm{w}), 917$ (s), $698(\mathrm{vs}) \mathrm{cm}^{-1}$.

HRMS (ESI [M+Na] $]^{+}$calcd for $\mathrm{C}_{20} \mathrm{H}_{28} \mathrm{NaO} 307.2038$, found 307.2048.

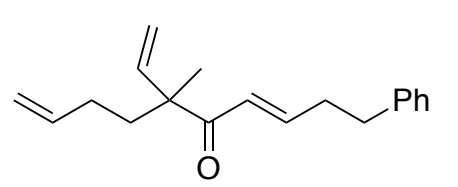

\section{(E)-6-Methyl-1-phenyl-6-vinyldeca-3,9-dien-5-one (3o)}

Color and State: Pale yellow oil; $b: l \geq 19: 1$

${ }^{1} \mathbf{H}$ NMR $\left(500 \mathrm{MHz}, \mathrm{CDCl}_{3}\right) \delta$ 7.29-7.26 (m, 2H), 7.21-7.15 (m, 3H), $6.94(\mathrm{dt}, J=15.3,7.0 \mathrm{~Hz}, 1 \mathrm{H}), 6.38(\mathrm{dt}, J=15.3,1.5 \mathrm{~Hz}, 1 \mathrm{H}), 5.86(\mathrm{dd}, J=17.5,10.8 \mathrm{~Hz}, 1 \mathrm{H}), 5.78$ (ddt, $J=16.9,10.4,6.5 \mathrm{~Hz}, 1 \mathrm{H}), 5.19$ (dd, $J=10.8,0.8 \mathrm{~Hz}, 1 \mathrm{H}), 5.11$ (dd, $J=17.5,0.8 \mathrm{~Hz}, 1 \mathrm{H}$ ), 4.99 (app. dq, $J=17.1,1.7 \mathrm{~Hz}, 1 \mathrm{H}), 4.95-4.92$ (m, 1H), 2.76 (t, $J=7.7 \mathrm{~Hz}, 2 \mathrm{H}), 2.53-2.49$ (m, 2H), 2.00-1.92 (m, 1H), 1.90-1.82 (m, 1H), $1.74\left(\mathrm{ddd}, \mathrm{A}\right.$ of $\mathrm{ABXY}, J_{\mathrm{AB}}=13.6 \mathrm{~Hz}, J_{\mathrm{AX}}=12.0 \mathrm{~Hz}, J_{\mathrm{AY}}=$ $4.8 \mathrm{~Hz}, 1 \mathrm{H}), 1.66\left(\mathrm{ddd}, \mathrm{B}\right.$ of $\left.\mathrm{ABXY}, J_{\mathrm{AB}}=13.6 \mathrm{~Hz}, J_{\mathrm{BX}}=12.1 \mathrm{~Hz}, J_{\mathrm{BY}}=5.0 \mathrm{~Hz}, 1 \mathrm{H}\right), 1.19(\mathrm{~s}, 3 \mathrm{H})$.

${ }^{13}$ C NMR (125 MHz, $\mathrm{CDCl}_{3}$ ) $\delta 200.86$ (e), 146.24 (o), 141.41 (o), 140.93 (e), 138.66 (o), 128.53 (o), 128.47 (o), 126.20 (o), 125.94 (o), 115.50 (e), 114.58 (e), 52.90 (e), 36.26 (e), 34.53 (e), 34.21 (e), 28.64 (e), 19.95 (o).

IR (Neat) 3083 (w), 3028 (w), 2976 (w), 2932 (w), 2857 (w), 1690 (s), 1623 (s), 1497 (w), 1454 (m), $1179(\mathrm{w}), 912$ (s), $698(\mathrm{~s}) \mathrm{cm}^{-1}$.

HRMS (ESI [M+Na] $]^{+}$) calcd for $\mathrm{C}_{19} \mathrm{H}_{24} \mathrm{NaO} 291.1725$, found 291.1721 . 
<smiles>C=CC(C)(CCOc1ccccc1)C(=O)/C=C/CCc1ccccc1</smiles>

(E)-3-(2-(Benzyloxy)ethyl)-3-methyl-8-phenylocta-1,5-dien-4-one (3p)

Color and State: Pale yellow oil; $b: l \geq 19: 1$

${ }^{1}$ H NMR $\left(500 \mathrm{MHz}, \mathrm{CDCl}_{3}\right) \delta$ 7.34-7.25 (m, 7H), 7.20-7.14 (m, 3H), $6.91(\mathrm{dt}, J=15.3,6.9 \mathrm{~Hz}, 1 \mathrm{H})$, 6.39 (dt, $J=15.3,1.5 \mathrm{~Hz}, 1 \mathrm{H}), 5.87$ (dd, $J=17.5,10.8 \mathrm{~Hz}, 1 \mathrm{H}), 5.18$ (dd, $J=10.8,0.7 \mathrm{~Hz}, 1 \mathrm{H}), 5.12$ (dd, $J=17.5,0.6 \mathrm{~Hz}, 1 \mathrm{H}), 4.43(\mathrm{~s}, 2 \mathrm{H}), 3.47$ (ddd, A of ABXY, $J_{\mathrm{AB}}=9.4 \mathrm{~Hz}, J_{\mathrm{AX}}=8.2 \mathrm{~Hz}, J_{\mathrm{AY}}=$ $5.7 \mathrm{~Hz}, 1 \mathrm{H}), 3.42\left(\mathrm{ddd}, \mathrm{B}\right.$ of ABXY, $\left.J_{\mathrm{AB}}=9.4 \mathrm{~Hz}, J_{\mathrm{BX}}=8.1 \mathrm{~Hz}, J_{\mathrm{BY}}=6.5 \mathrm{~Hz}, 1 \mathrm{H}\right), 2.73(\mathrm{t}, J=7.7$ $\mathrm{Hz}, 2 \mathrm{H}), 2.50-2.46(\mathrm{~m}, 2 \mathrm{H}), 2.07$ (ddd, A of ABXY, $J_{\mathrm{AB}}=14.1 \mathrm{~Hz}, J_{\mathrm{AX}}=8.0 \mathrm{~Hz}, J_{\mathrm{AY}}=6.3 \mathrm{~Hz}, 1 \mathrm{H}$ ), $1.95\left(\mathrm{ddd}, \mathrm{B}\right.$ of $\left.\mathrm{ABXY}, J_{\mathrm{AB}}=13.8 \mathrm{~Hz}, J_{\mathrm{BX}}=8.1 \mathrm{~Hz}, J_{\mathrm{BY}}=5.7 \mathrm{~Hz}, 1 \mathrm{H}\right), 1.21(\mathrm{~s}, 3 \mathrm{H})$.

${ }^{13}$ C NMR (125 MHz, $\mathrm{CDCl}_{3}$ ) $\delta 200.40$ (e), 146.08 (o), 141.31 (o), 140.97 (e), 138.48 (e), 128.52 (o), 128.46 (o), 128.40 (o), 127.68 (o), 127.59 (o), 126.18 (o), 125.86 (o), 115.46 (e), 73.07 (e), 66.93 (e), 51.80 (e), 36.66 (e), 34.50 (e), 34.19 (e), 20.44 (o).

IR (Neat) 3086 (w), 3063 (w), 3028 (w), 2932 (w), 2858 (w), 1690 (m), 1623 (m), 1496 (w), 1454 (m), 1097 (m), 919 (m), 697 (vs) $\mathrm{cm}^{-1}$.

HRMS (ESI [M+Na] $]^{+}$) calcd for $\mathrm{C}_{24} \mathrm{H}_{28} \mathrm{NaO}_{2} 371.1987$, found 371.1972 .

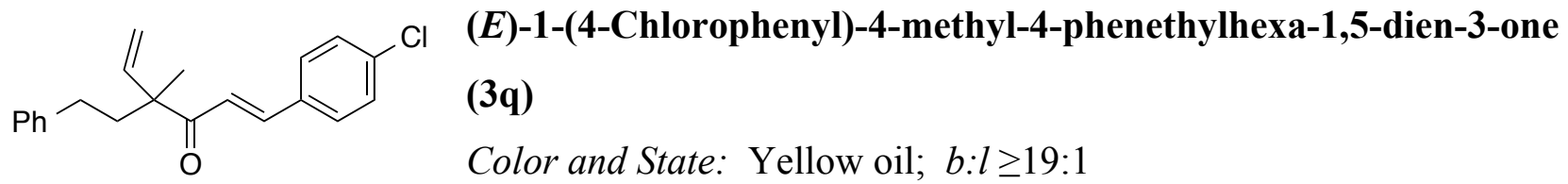

${ }^{1} \mathbf{H}$ NMR $\left(500 \mathrm{MHz}, \mathrm{CDCl}_{3}\right) \delta 7.62(\mathrm{~d}, J=15.7 \mathrm{~Hz}, 1 \mathrm{H}), 7.49-7.47(\mathrm{~m}, 2 \mathrm{H}), 7.37-7.34(\mathrm{~m}, 2 \mathrm{H})$, 7.28-7.25 (m, 2H), 7.19-7.16 (m, 3H), 7.03 (d, $J=15.7 \mathrm{~Hz}, 1 \mathrm{H}), 6.02$ (dd, $J=17.5,10.8 \mathrm{~Hz}, 1 \mathrm{H})$, $5.31(\mathrm{dd}, J=10.7,0.8 \mathrm{~Hz}, 1 \mathrm{H}), 5.26(\mathrm{dd}, J=17.6,0.6 \mathrm{~Hz}, 1 \mathrm{H}), 2.59\left(\mathrm{dt}, \mathrm{A}\right.$ of $\mathrm{ABX}_{2}, J_{A B}=12.9 \mathrm{~Hz}$, $\left.J_{A X}=5.0 \mathrm{~Hz}, 1 \mathrm{H}\right), 2.47\left(\mathrm{dt}, \mathrm{B}\right.$ of $\left.\mathrm{ABX}_{2}, J_{A B}=13.0 \mathrm{~Hz}, J_{B X}=5.0 \mathrm{~Hz}, 1 \mathrm{H}\right), 2.07\left(\mathrm{dt}, \mathrm{A}\right.$ of $\mathrm{ABX}_{2}, J_{A B}=$ $\left.13.1 \mathrm{~Hz}, J_{A X}=5.0 \mathrm{~Hz}, 1 \mathrm{H}\right), 1.98\left(\mathrm{dt}, \mathrm{B}\right.$ of $\left.\mathrm{ABX}_{2}, J_{A B}=13.1 \mathrm{~Hz}, J_{B X}=5.0 \mathrm{~Hz}, 1 \mathrm{H}\right), 1.37(\mathrm{~s}, 3 \mathrm{H})$.

${ }^{13}$ C NMR (125 MHz, CDCl $) \delta 200.67$ (e), 142.36 (e), 141.58 (o), 141.25 (o), 136.30 (e), 133.39 (e), 129.66 (o), 129.26 (o), 128.55 (o), 128.46 (o), 126.03 (o), 122.23 (o), 116.19 (e), 53.58 (e), 39.29 (e), 30.91 (e), 20.25 (o).

IR (Neat) 3085 (w), 3063 (w), 3029 (w), 2954 (w), 2852 (w), 1679 (s), 1633 (w), 1608 (s), 1490 (m), $1456(\mathrm{~m}), 1091(\mathrm{w}), 928(\mathrm{~m}), 821(\mathrm{~s}) \mathrm{cm}^{-1}$.

HRMS (ESI $[\mathrm{M}+\mathrm{Na}]^{+}$) calcd for $\mathrm{C}_{21} \mathrm{H}_{21}{ }^{35} \mathrm{ClNaO} 347.1179$, found 347.1163 . 


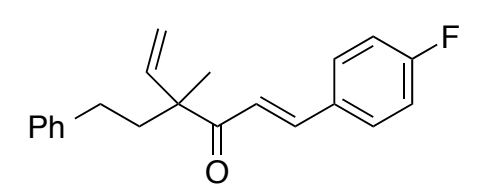

(E)-1-(4-Fluorophenyl)-4-methyl-4-phenethylhexa-1,5-dien-3-one (3r)

Color and State: Colorless oil; $b: l \geq 19: 1$

${ }^{1}$ H NMR $\left(500 \mathrm{MHz}, \mathrm{CDCl}_{3}\right) \delta 7.67(\mathrm{~d}, J=15.7 \mathrm{~Hz}, 1 \mathrm{H}), 7.57-7.53(\mathrm{~m}, 2 \mathrm{H}), 7.30-7.27(\mathrm{~m}, 2 \mathrm{H})$, 7.20-7.17 (m, 3H), 7.11-7.06 (m, 2H), $7.02(\mathrm{~d}, J=15.7 \mathrm{~Hz}, 1 \mathrm{H}), 6.05(\mathrm{dd}, J=17.6,10.7 \mathrm{~Hz}, 1 \mathrm{H})$, $5.33(\mathrm{dd}, J=10.8,0.7 \mathrm{~Hz}, 1 \mathrm{H}), 5.28(\mathrm{dd}, J=17.5,0.7 \mathrm{~Hz}, 1 \mathrm{H}), 2.61\left(\mathrm{dt}, \mathrm{A}\right.$ of $\mathrm{ABX}_{2}, J_{A B}=12.9 \mathrm{~Hz}$, $\left.J_{A X}=5.0 \mathrm{~Hz}, 1 \mathrm{H}\right), 2.51\left(\mathrm{dt}, \mathrm{B}\right.$ of $\left.\mathrm{ABX}_{2}, J_{A B}=13.0 \mathrm{~Hz}, J_{B X}=5.1 \mathrm{~Hz}, 1 \mathrm{H}\right), 2.10\left(\mathrm{dt}, \mathrm{A}\right.$ of $\mathrm{ABX}_{2}, J_{A B}=$ $\left.13.0 \mathrm{~Hz}, J_{A X}=5.1 \mathrm{~Hz}, 1 \mathrm{H}\right), 2.01\left(\mathrm{dt}, \mathrm{B}\right.$ of $\left.\mathrm{ABX}_{2}, J_{A B}=13.1 \mathrm{~Hz}, J_{B X}=5.0 \mathrm{~Hz}, 1 \mathrm{H}\right), 1.39(\mathrm{~s}, 3 \mathrm{H})$.

${ }^{13}$ C NMR (125 MHz, $\left.\mathrm{CDCl}_{3}\right) \delta 200.64$ (e), 164.04 (e, d, $\left.{ }^{l} J_{C-F}=251.5 \mathrm{~Hz}\right), 142.40$ (e), 141.73 (o), 141.38 (o), 131.18 (e, d, ${ }^{4} J_{C-F}=3.4 \mathrm{~Hz}$ ), 130.37 (o, d, ${ }^{3} J_{C-F}=8.8 \mathrm{~Hz}$ ), 128.53 (o), 128.45 (o), 126.00 (o), $121.58\left(\mathrm{o}, \mathrm{d},{ }^{5} J_{C-F}=2.0 \mathrm{~Hz}\right), 116.13\left(\mathrm{o}, \mathrm{d},{ }^{2} J_{C-F}=21.1 \mathrm{~Hz}\right), 116.02$ (e), 53.54 (e), 39.35 (e), 30.92 (e), 20.29 (o).

IR (Neat) 3085 (w), 3062 (w), 3028 (w), 2975 (w), 2936 (w), 2866 (w), 1685 (m), 1612 (m), 1599 (s), 1590 (m), 1509 (vs), 1455 (w), 1233 (m), 1159 (m), 923 (w), 700 (m) cm c $^{-1}$.

HRMS (EI, $\mathrm{M}^{+}$) calcd for $\mathrm{C}_{21} \mathrm{H}_{21} \mathrm{FO} 308.1576$, found 308.1581.

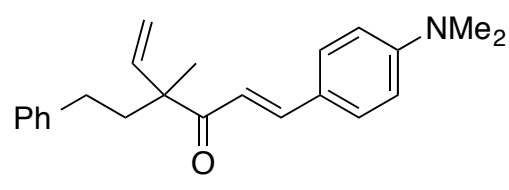

(E)-1-(4-(Dimethylamino)phenyl)-4-methyl-4-phenethylhexa-1,5dien-3-one (3s)

Color and State: Orange solid; $\mathbf{m p}=90-92{ }^{\circ} \mathrm{C} ; b: l \geq 19: 1$

${ }^{1}$ H NMR $\left(500 \mathrm{MHz}, \mathrm{CDCl}_{3}\right) \delta 7.67(\mathrm{~d}, J=15.5 \mathrm{~Hz}, 1 \mathrm{H}), 7.48-7.45(\mathrm{~m}, 2 \mathrm{H}), 7.28-7.25(\mathrm{~m}, 3 \mathrm{H})$, 7.18-7.15 (m, 2H), $6.88(\mathrm{~d}, J=15.4 \mathrm{~Hz}, 1 \mathrm{H}), 6.68-6.65(\mathrm{~m}, 2 \mathrm{H}), 6.06(\mathrm{dd}, J=17.6,10.8 \mathrm{~Hz}, 1 \mathrm{H})$, $5.26(\mathrm{dd}, J=10.7,0.9 \mathrm{~Hz}, 1 \mathrm{H}), 5.24(\mathrm{dd}, J=17.5,0.9 \mathrm{~Hz}, 1 \mathrm{H}), 3.03(\mathrm{~s}, 6 \mathrm{H}), 2.58\left(\mathrm{dt}, \mathrm{A}\right.$ of $\mathrm{ABX}_{2}$, $\left.J_{A B}=13.0 \mathrm{~Hz}, J_{A X}=5.0 \mathrm{~Hz}, 1 \mathrm{H}\right), 2.49\left(\mathrm{dt}, \mathrm{B}\right.$ of $\left.\mathrm{ABX}_{2}, J_{A B}=13.0 \mathrm{~Hz}, J_{B X}=4.9 \mathrm{~Hz}, 1 \mathrm{H}\right), 2.07(\mathrm{dt}, \mathrm{A}$ of $\left.\mathrm{ABX}_{2}, J_{A B}=13.1 \mathrm{~Hz}, J_{A X}=5.0 \mathrm{~Hz}, 1 \mathrm{H}\right), 1.97\left(\mathrm{dt}, \mathrm{B}\right.$ of $\left.\mathrm{ABX}_{2}, J_{A B}=13.1 \mathrm{~Hz}, J_{B X}=5.0 \mathrm{~Hz}, 1 \mathrm{H}\right)$, $1.36(\mathrm{~s}, 3 \mathrm{H})$.

${ }^{13}$ C NMR (125 MHz, $\mathrm{CDCl}_{3}$ ) $\delta 200.87$ (e), 151.96 (e), 143.84 (o), 142.70 (e), 142.10 (o), 130.30 (o), 128.45 (o), 125.84 (o), 122.63 (e), 116.74 (o), 115.14 (e), 111.87 (o), 53.29 (e), 40.20 (o), 39.70 (e), 31.00 (e), 20.52 (o).

IR (Neat) 3085 (w), 3060 (w), 3025 (w), 2930 (w), 2862 (w), 1673 (w), 1579 (vs), 1524 (s), 1498 (w), $1446(\mathrm{w}), 1184(\mathrm{~m}), 1062(\mathrm{~m}), 919(\mathrm{w}), 701(\mathrm{w}) \mathrm{cm}^{-1}$.

HRMS (EI, $\mathrm{M}^{+}$) calcd for $\mathrm{C}_{23} \mathrm{H}_{27} \mathrm{NO} 333.2093$, found 333.2090. 


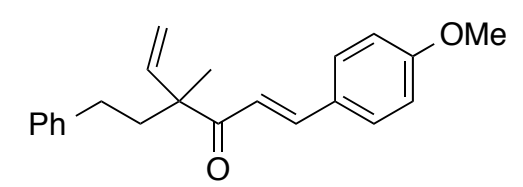

\section{(E)-1-(4-Methoxyphenyl)-4-methyl-4-phenethylhexa-1,5-dien-3-} one (3t)

Color and State: Yellow oil; $b: l \geq 19: 1$

${ }^{1}$ H NMR $\left(500 \mathrm{MHz}, \mathrm{CDCl}_{3}\right) \delta 7.66(\mathrm{~d}, J=15.6 \mathrm{~Hz}, 1 \mathrm{H}), 7.53-7.50(\mathrm{~m}, 2 \mathrm{H}), 7.28-7.25(\mathrm{~m}, 2 \mathrm{H})$, 7.18-7.16 (m, 3H), $6.95(\mathrm{~d}, J=15.6 \mathrm{~Hz}, 1 \mathrm{H}), 6.92-6.89(\mathrm{~m}, 2 \mathrm{H}), 6.03(\mathrm{dd}, J=17.5,10.8 \mathrm{~Hz}, 1 \mathrm{H})$, $5.29(\mathrm{~d}, J=10.7 \mathrm{~Hz}, 1 \mathrm{H}), 5.25(\mathrm{~d}, J=17.6 \mathrm{~Hz}, 1 \mathrm{H}), 3.84(\mathrm{~s}, 3 \mathrm{H}), 2.58\left(\mathrm{dt}, \mathrm{A}\right.$ of $\mathrm{ABX}_{2}, J_{A B}=13.0$ $\left.\mathrm{Hz}, J_{A X}=4.9 \mathrm{~Hz}, 1 \mathrm{H}\right), 2.48\left(\mathrm{dt}, \mathrm{B}\right.$ of $\left.\mathrm{ABX}_{2}, J_{A B}=13.0 \mathrm{~Hz}, J_{B X}=4.9 \mathrm{~Hz}, 1 \mathrm{H}\right), 2.07\left(\mathrm{dt}, \mathrm{A}\right.$ of $\mathrm{ABX}_{2}$, $\left.J_{A B}=13.1 \mathrm{~Hz}, J_{A X}=5.0 \mathrm{~Hz}, 1 \mathrm{H}\right), 1.97\left(\mathrm{dt}, \mathrm{B}\right.$ of $\left.\mathrm{ABX}_{2}, J_{A B}=13.1 \mathrm{~Hz}, J_{B X}=4.9 \mathrm{~Hz}, 1 \mathrm{H}\right), 1.36(\mathrm{~s}, 3 \mathrm{H})$. ${ }^{13}$ C NMR (125 MHz, $\mathrm{CDCl}_{3}$ ) $\delta 200.87$ (e), 161.54 (e), 142.81 (o), 142.49 (e), 141.59 (o), 130.21 (o), 128.48 (o), 128.44 (o), 127.54 (e), 125.92 (o), 119.46 (o), 115.70 (e), 114.38 (o), 55.46 (o), 53.42 (e), 39.42 (e), 30.91 (e), 20.32 (o).

IR (Neat) 3061 (w), 3027 (w), 2934 (w), 2838 (w), 1678 (m), 1633 (w), 1594 (vs), 1572 (s), 1510 (s), 1455 (m), 1253 (s), 1172 (s), 1031 (s), 920 (m), 825 (m), 700 (m) cm cm $^{-1}$

HRMS (ESI $[\mathrm{M}+\mathrm{Na}]^{+}$) calcd for $\mathrm{C}_{22} \mathrm{H}_{24} \mathrm{NaO}_{2} 343.1674$, found 343.1660.

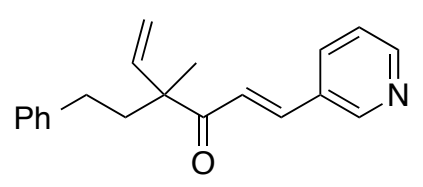

\section{(E)-4-Methyl-4-phenethyl-1-(pyridin-3-yl)hexa-1,5-dien-3-one (3u)}

Color and State: Yellow oil; $b: l \geq 19: 1$

${ }^{1}$ H NMR $\left(500 \mathrm{MHz}, \mathrm{CDCl}_{3}\right) \delta 8.77(\mathrm{~s}, 1 \mathrm{H}), 8.60(\mathrm{~d}, J=4.2 \mathrm{~Hz}, 1 \mathrm{H}), 7.86$ (dt, $J=7.9,1.8 \mathrm{~Hz}, 1 \mathrm{H}), 7.65(\mathrm{~d}, J=15.8 \mathrm{~Hz}, 1 \mathrm{H}), 7.34(\mathrm{dd}, J=7.9,4.8 \mathrm{~Hz}, 1 \mathrm{H}), 7.28-7.25(\mathrm{~m}, 2 \mathrm{H})$, 7.18-7.16 (m, 3H), 7.12 (d, $J=15.8 \mathrm{~Hz}, 1 \mathrm{H}), 6.01(\mathrm{dd}, J=17.5,10.8 \mathrm{~Hz}, 1 \mathrm{H}), 5.33$ (d, $J=10.7 \mathrm{~Hz}$, $1 \mathrm{H}), 5.27(\mathrm{~d}, J=17.5 \mathrm{~Hz}, 1 \mathrm{H}), 2.60\left(\mathrm{dt}, \mathrm{A}\right.$ of $\left.\mathrm{ABX}_{2}, J_{A B}=12.9 \mathrm{~Hz}, J_{A X}=5.0 \mathrm{~Hz}, 1 \mathrm{H}\right), 2.48(\mathrm{dt}, \mathrm{B}$ of $\left.\mathrm{ABX}_{2}, J_{A B}=12.9 \mathrm{~Hz}, J_{B X}=5.1 \mathrm{~Hz}, 1 \mathrm{H}\right), 2.08\left(\mathrm{dt}, \mathrm{A}\right.$ of $\left.\mathrm{ABX}_{2}, J_{A B}=13.0 \mathrm{~Hz}, J_{A X}=5.1 \mathrm{~Hz}, 1 \mathrm{H}\right), 1.99$ $\left(\mathrm{dt}, \mathrm{B}\right.$ of $\left.\mathrm{ABX}_{2}, J_{A B}=13.1 \mathrm{~Hz}, J_{B X}=5.0 \mathrm{~Hz}, 1 \mathrm{H}\right), 1.38(\mathrm{~s}, 3 \mathrm{H})$.

${ }^{13}$ C NMR (125 MHz, $\mathrm{CDCl}_{3}$ ) $\delta 200.25$ (e), 151.01 (o), 150.01 (o), 142.22 (e), 141.05 (o), 139.18 (o), 134.64 (o), 130.67 (e), 128.52 (o), 128.42 (o), 126.03 (o), 123.77 (o), 123.66 (o), 116.38 (e), 53.57 (e), 39.18 (e), 30.85 (e), 20.19 (o).

IR (Neat) 3084 (w), 3060 (w), 3027 (w), 2975 (w), 2932 (w), 2863 (w), 1685 (s), 1610 (s), 1586 (m), 1567 (m), 1497 (w), 1455 (m), 1296 (m), 1124 (w), 1024 (m), 921 (m), 699 (s) cm cm $^{-1}$.

HRMS (ESI [M+Na] $]^{+}$) calcd for $\mathrm{C}_{20} \mathrm{H}_{21} \mathrm{NNaO} 314.1521$, found 314.1529. 


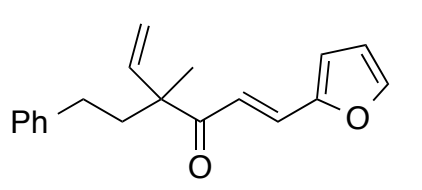

\section{(E)-1-(Furan-2-yl)-4-methyl-4-phenethylhexa-1,5-dien-3-one (3v)}

Color and State: Yellow oil; $b: l \geq 19: 1$

${ }^{1}$ H NMR $\left(500 \mathrm{MHz}, \mathrm{CDCl}_{3}\right) \delta 7.48(\mathrm{~d}, J=1.5 \mathrm{~Hz}, 1 \mathrm{H}), 7.44(\mathrm{~d}, J=15.4$ $\mathrm{Hz}, 1 \mathrm{H}), 7.28-7.25(\mathrm{~m}, 2 \mathrm{H}), 7.18-7.16(\mathrm{~m}, 3 \mathrm{H}), 6.95(\mathrm{~d}, J=15.4 \mathrm{~Hz}, 1 \mathrm{H}), 6.65(\mathrm{~d}, J=3.4 \mathrm{~Hz}, 1 \mathrm{H})$, $6.48(\mathrm{dd}, J=3.4,1.8 \mathrm{~Hz}, 1 \mathrm{H}), 6.02(\mathrm{dd}, J=17.5,10.8 \mathrm{~Hz}, 1 \mathrm{H}), 5.29$ (d, $J=10.8 \mathrm{~Hz}, 1 \mathrm{H}), 5.25(\mathrm{~d}, J$ $=17.8 \mathrm{~Hz}, 1 \mathrm{H}), 2.57\left(\mathrm{dt}, \mathrm{A}\right.$ of $\left.\mathrm{ABX}_{2}, J_{A B}=13.0 \mathrm{~Hz}, J_{A X}=4.9 \mathrm{~Hz}, 1 \mathrm{H}\right), 2.47\left(\mathrm{dt}, \mathrm{B}\right.$ of $\mathrm{ABX}_{2}, J_{A B}=$ $\left.13.0 \mathrm{~Hz}, J_{B X}=4.9 \mathrm{~Hz}, 1 \mathrm{H}\right), 2.07\left(\mathrm{dt}, \mathrm{A}\right.$ of $\left.\mathrm{ABX}_{2}, J_{A B}=13.1 \mathrm{~Hz}, J_{A X}=5.0 \mathrm{~Hz}, 1 \mathrm{H}\right), 1.96(\mathrm{dt}, \mathrm{B}$ of $\left.\mathrm{ABX}_{2}, J_{A B}=13.1 \mathrm{~Hz}, J_{B X}=4.9 \mathrm{~Hz}, 1 \mathrm{H}\right), 1.36(\mathrm{~s}, 3 \mathrm{H})$.

${ }^{13}$ C NMR (125 MHz, $\mathrm{CDCl}_{3}$ ) $\delta 200.74$ (e), 151.62 (e), 144.75 (o), 142.45 (e), 141.35 (o), 129.21 (o), 128.49 (o), 128.45 (o), 125.95 (o), 119.43 (o), 115.98 (o), 115.90 (e), 112.64 (o), 53.40 (e), 39.38 (e), 30.90 (e), 20.21 (o).

IR (Neat) 3085 (w), 3026 (w), 2974 (w), 2933 (w), 2863 (w), 1680 (m), 1604 (s), 1552 (m), 1497 (w), $1455(\mathrm{w}), 1126(\mathrm{w}), 1015$ (s), 925 (w), 699 (m) $\mathrm{cm}^{-1}$.

HRMS (ESI, $\mathrm{M}^{+}$) calcd for $\mathrm{C}_{19} \mathrm{H}_{20} \mathrm{O}_{2} 280.1463$, found 280.1469 .

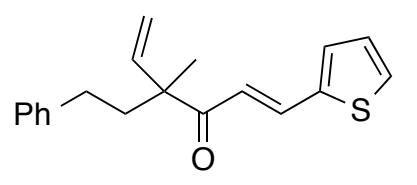

(E)-4-Methyl-4-phenethyl-1-(thiophenol-2-yl)hexa-1,5-dien-3-one (3w)

Color and State: Yellow oil; $b: l \geq 19: 1$

${ }^{1} \mathbf{H}$ NMR $\left(500 \mathrm{MHz}, \mathrm{CDCl}_{3}\right) \delta 7.80(\mathrm{~d}, J=15.3 \mathrm{~Hz}, 1 \mathrm{H}), 7.37$ (d, $J=5.1$ $\mathrm{Hz}, 1 \mathrm{H}), 7.29$ (d, $J=3.7 \mathrm{~Hz}, 1 \mathrm{H}), 7.28-7.25(\mathrm{~m}, 2 \mathrm{H}), 7.18-7.16(\mathrm{~m}, 3 \mathrm{H}), 7.06(\mathrm{dd}, J=5.1,3.6 \mathrm{~Hz}$, 1H), $6.85(\mathrm{~d}, J=15.3 \mathrm{~Hz}, 1 \mathrm{H}), 6.02(\mathrm{dd}, J=17.5,10.8 \mathrm{~Hz}, 1 \mathrm{H}), 5.29$ (dd, $J=10.7,0.8 \mathrm{~Hz}, 1 \mathrm{H}), 5.24$ $(\mathrm{dd}, J=17.5,0.7 \mathrm{~Hz}, 1 \mathrm{H}), 2.58\left(\mathrm{dt}, \mathrm{A}\right.$ of $\left.\mathrm{ABX}_{2}, J_{A B}=12.9 \mathrm{~Hz}, J_{A X}=5.0 \mathrm{~Hz}, 1 \mathrm{H}\right), 2.48(\mathrm{dt}, \mathrm{B}$ of $\left.\mathrm{ABX}_{2}, J_{A B}=13.0 \mathrm{~Hz}, J_{B X}=5.0 \mathrm{~Hz}, 1 \mathrm{H}\right), 2.06\left(\mathrm{ddd}, \mathrm{A}\right.$ of $\mathrm{ABXY}, J_{\mathrm{AB}}=13.7 \mathrm{~Hz}, J_{\mathrm{AX}}=12.4 \mathrm{~Hz}, J_{\mathrm{AY}}$ $=5.0 \mathrm{~Hz}, 1 \mathrm{H}), 1.96\left(\mathrm{ddd}, \mathrm{B}\right.$ of $\left.\mathrm{ABXY}, J_{\mathrm{AB}}=13.7 \mathrm{~Hz}, J_{\mathrm{BX}}=12.5 \mathrm{~Hz}, J_{\mathrm{BY}}=5.0 \mathrm{~Hz}, 1 \mathrm{H}\right), 1.36(\mathrm{~s}, 3 \mathrm{H})$.

${ }^{13}$ C NMR (125 MHz, $\mathrm{CDCl}_{3}$ ) $\delta 200.52$ (e), 142.43 (e), 141.36 (o), 140.38 (e), 135.52 (o), 131.81 (o), 128.52 (o), 128.49 (o), 128.47 (o), 128.35 (o), 125.98 (o), 120.77 (o), 115.92 (e), 53.38 (e), 39.44 (e), 30.94 (e), 20.30 (o).

IR (Neat) 3084 (w), 3025 (w), 2973 (w), 2931 (w), 2863 (w), 1678 (m), 1632 (w), 1591 (s), 1497 (w), $1455(\mathrm{w}), 1126(\mathrm{w}), 1033(\mathrm{~m}), 921(\mathrm{~m}), 700(\mathrm{~m}) \mathrm{cm}^{-1}$.

HRMS (ESI [M+Na] $]^{+}$calcd for $\mathrm{C}_{19} \mathrm{H}_{20} \mathrm{NaOS} 319.1133$, found 319.1132. 


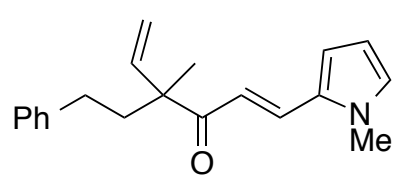

(E)-4-Methyl-1-(1-methyl-1H-pyrrol-2-yl)-4-phenethylhexa-1,5-dien-3one (3x)

Color and State: Yellow oil; $b: l \geq 19: 1$

${ }^{1} \mathbf{H}$ NMR $\left(500 \mathrm{MHz}, \mathrm{CDCl}_{3}\right) \delta 7.65(\mathrm{~d}, J=15.3 \mathrm{~Hz}, 1 \mathrm{H}), 7.28-7.25(\mathrm{~m}, 2 \mathrm{H}), 7.19-7.16(\mathrm{~m}, 3 \mathrm{H}), 6.80$ (d, $J=15.3 \mathrm{~Hz}, 1 \mathrm{H}), 6.77$ (t, $J=2.0 \mathrm{~Hz}, 1 \mathrm{H}), 6.73$ (dd, $J=3.8,1.5 \mathrm{~Hz}, 1 \mathrm{H}), 6.18$ (ddd, $J=3.9,2.6$, $0.5 \mathrm{~Hz}, 1 \mathrm{H}), 6.03(\mathrm{dd}, J=17.5,10.7 \mathrm{~Hz}, 1 \mathrm{H}), 5.26$ (dd, $J=10.8,0.9 \mathrm{~Hz}, 1 \mathrm{H}), 5.23$ (dd, $J=17.5,0.9$ $\mathrm{Hz}, 1 \mathrm{H}), 3.72(\mathrm{~s}, 3 \mathrm{H}), 2.58\left(\mathrm{dt}, \mathrm{A}\right.$ of $\left.\mathrm{ABX}_{2}, J_{A B}=13.0 \mathrm{~Hz}, J_{A X}=4.9 \mathrm{~Hz}, 1 \mathrm{H}\right), 2.49$ (dt, B of $\mathrm{ABX}_{2}$, $\left.J_{A B}=13.1 \mathrm{~Hz}, J_{B X}=5.0 \mathrm{~Hz}, 1 \mathrm{H}\right), 2.06\left(\mathrm{dt}, \mathrm{A}\right.$ of $\left.\mathrm{ABX}_{2}, J_{A B}=13.0 \mathrm{~Hz}, J_{A X}=5.0 \mathrm{~Hz}, 1 \mathrm{H}\right), 1.96(\mathrm{dt}, \mathrm{B}$ of $\left.\mathrm{ABX}_{2}, J_{A B}=13.1 \mathrm{~Hz}, J_{B X}=5.0 \mathrm{~Hz}, 1 \mathrm{H}\right), 1.35(\mathrm{~s}, 3 \mathrm{H})$.

${ }^{13}$ C NMR (125 MHz, $\mathrm{CDCl}_{3}$ ) $\delta 200.86$ (e), 142.65 (e), 141.94 (o), 130.63 (o), 130.09 (e), 128.49 (o), 128.47 (o), 127.51 (o), 125.91 (o), 117.04 (o), 115.32 (e), 112.07 (o), 109.62 (o), 53.22 (e), 39.67 (e), 34.43 (o), 31.02 (e), 20.52 (o).

IR (Neat) 3084 (w), 3060 (w), 3025 (w), 2932 (w), 2864 (w), 1701 (m), 1670 (m), 1583 (vs), 1482 (m), $1454(\mathrm{~m}), 1271(\mathrm{~m}), 1148(\mathrm{w}), 1054(\mathrm{~m}), 920(\mathrm{w}), 701(\mathrm{~m}) \mathrm{cm}^{-1}$.

HRMS (EI, M ${ }^{+}$) calcd for $\mathrm{C}_{20} \mathrm{H}_{23} \mathrm{NO} 293.1780$, found 293.1788.

\section{Spectral Data for the Enantiomerically Enriched Tertiary Allylic Alcohols 5a-c}

The enantiomerically enriched tertiary allylic alcohols were prepared according the previously reported procedure. ${ }^{3}$

Color and State: Colorless oil.
All spectral data matched the published values. ${ }^{3 c}$
$[\alpha]_{\mathrm{D}}^{20}+25.0\left(c=1.0, \mathrm{CHCl}_{3}\right),\left[\mathrm{lit}^{3 \mathrm{c}}[\alpha]_{\mathrm{D}}^{20}+27.2\left(c=1.0, \mathrm{CHCl}_{3}\right)\right]$; Chiral HPLC analysis (CHIRALCEL OJ-H column), 94:6 hexane/isopropanol at $1.0 \mathrm{~mL} / \mathrm{min}$ flow rate; $t_{\mathrm{R}}(S)$-enantiomer $($ minor $)=30.3$ min., $t_{\mathrm{R}}(R)$-enantiomer $($ major $)=39.6$ min.; 99\% ee .

${ }^{1} \mathbf{H}$ NMR $\left(500 \mathrm{MHz}, \mathrm{CDCl}_{3}\right) \delta$ 7.41-7.38 (m, 2H), 6.89-6.86 (m, 2H), $6.16(\mathrm{dd}, J=17.2,10.6 \mathrm{~Hz}$, $1 \mathrm{H}), 5.28(\mathrm{dd}, J=17.3,1.2 \mathrm{~Hz}, 1 \mathrm{H}), 5.13(\mathrm{dd}, J=10.6,1.2 \mathrm{~Hz}, 1 \mathrm{H}), 3.80$ (s, $3 \mathrm{H}), 1.81$ (br. s, $1 \mathrm{H}$ ), $1.64(\mathrm{~s}, 3 \mathrm{H})$.

IR (Neat) 3427 (br, w), 2977 (w), 2936 (w), 2836 (w), 1641 (w), 1610 (m), 1509 (s), 1245 (s), 919 (s), 829 (vs) $\mathrm{cm}^{-1}$. 


\section{(R)-2-Phenylbut-3-en-2-ol (5b)}

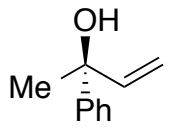

Color and State: Colorless oil.

All spectral data matched the published values. ${ }^{3 a}$

$[\alpha]_{\mathrm{D}}^{20}+22.2\left(c=1.0, \mathrm{CHCl}_{3}\right),\left[\right.$ lit. $\left.^{3 \mathrm{c}}[\alpha]_{\mathrm{D}}^{20}+24.6\left(c=1.0, \mathrm{CHCl}_{3}\right)\right] ;$ Chiral HPLC analysis (CHIRALCEL OJ-H column), 98:2 hexane/isopropanol at $1.0 \mathrm{~mL} / \mathrm{min}$ flow rate; $t_{\mathrm{R}}(S)$-enantiomer $($ minor $)=26.3$ min., $t_{\mathrm{R}}(R)$-enantiomer $($ major $)=31.1 \mathrm{~min} . ;>99 \%$ ee.

${ }^{1}$ H NMR $\left(500 \mathrm{MHz}, \mathrm{CDCl}_{3}\right) \delta$ 7.49-7.46 (m, 2H), 7.36-7.33 (m, 2H), 7.27-7.24 (m, 1H), 6.18 (dd, J $=17.3,10.6 \mathrm{~Hz}, 1 \mathrm{H}), 5.30(\mathrm{dd}, J=17.2,1.0 \mathrm{~Hz}, 1 \mathrm{H}), 5.15$ (dd, $J=10.6,1.0 \mathrm{~Hz}, 1 \mathrm{H}), 1.87$ (br. s, $1 \mathrm{H}), 1.66(\mathrm{~s}, 3 \mathrm{H})$.

IR (Neat) 3397 (br, w), 3086 (w), 3061 (w), 3028 (w), 2979 (w), 2934 (w), 1639 (w), 1601 (w), 1492 (w), 1446 (m), 921 (s), 765 (s), $698(\mathrm{vs}) \mathrm{cm}^{-1}$.

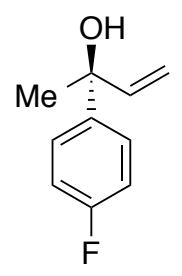

(R)-2-(4-Fluorophenyl)but-3-en-2-ol (5c)

Color and State: Colorless oil.

All spectral data matched the published values. ${ }^{3 \mathrm{c}}$

$[\alpha]_{\mathrm{D}}^{20}+16.9\left(c=1.0, \mathrm{CHCl}_{3}\right),\left[\right.$ lit. $\left.^{3 \mathrm{c}}[\alpha]_{\mathrm{D}}^{20}+10.8\left(c=1.0, \mathrm{CHCl}_{3}\right)\right]$; Chiral HPLC analysis

(CHIRALCEL OJ-H column), 98:2 hexane/isopropanol at $0.6 \mathrm{~mL} / \mathrm{min}$ flow rate; $t_{\mathrm{R}}(S)$-enantiomer $($ minor $)=29.0$ min., $t_{\mathrm{R}}(R)$-enantiomer $($ major $)=39.1 \mathrm{~min} . ;>99 \%$ ee.

${ }^{1}$ H NMR $\left(500 \mathrm{MHz}, \mathrm{CDCl}_{3}\right) \delta$ 7.46-7.42 (m, 2H), 7.04-6.99 (m, 2H), $6.15(\mathrm{dd}, J=17.3,10.6 \mathrm{~Hz}$, 1H), 5.29 (dd, $J=17.3,1.0 \mathrm{~Hz}, 1 \mathrm{H}), 5.15$ (dd, $J=10.6,0.9 \mathrm{~Hz}, 1 \mathrm{H}), 1.83$ (br. s, $1 \mathrm{H}), 1.65$ (s, $3 \mathrm{H})$.

IR (Neat) 3397 (br, w), 3087 (w), 2983 (w), 2932 (w), 1643 (w), 1601 (m), 1507 (s), 1223 (s), 921 (s), $834(\mathrm{vs}), 729(\mathrm{~m}) \mathrm{cm}^{-1}$.

\section{Representative Experimental Procedure for the Stereospecific Rhodium-Catalyzed Allylic} Substitution with Alkenyl Cyanohydrin Pronucleophiles

$n$-Butyllithium $(0.230 \mathrm{~mL}, 0.575 \mathrm{mmol} ; 2.5 \mathrm{M}$ solution in hexane) was added dropwise to a stirred solution of $(R)-2$-phenylbut-3-en-2-ol $\mathbf{5 b}(74.0 \mathrm{mg}, 0.500 \mathrm{mmol})$ in anhydrous tetrahydrofuran $(1.5$ $\mathrm{mL}$ ) at $0{ }^{\circ} \mathrm{C}$ under an atmosphere of argon. The mixture was stirred for $c a .15$ minutes and methyl chloroformate $(0.044 \mathrm{~mL}, 0.575 \mathrm{mmol})$ was added dropwise. The mixture was then allowed to warm to room temperature and stirred for $c a$. 1 hour. In a separate flask, lithium bis(trimethylsilyl)amide $(1.40 \mathrm{~mL}, 1.4 \mathrm{mmol}$; $1 \mathrm{M}$ solution in tetrahydrofuran) was added dropwise to a solution of $(E)-2-$ 
((tert-butyldimethylsilyl)oxy)-6-phenylhex-3-enenitrile 2a $(301.0 \mathrm{mg}, 1.0 \mathrm{mmol})$ in anhydrous tetrahydrofuran $(2 \mathrm{~mL})$ at $-10{ }^{\circ} \mathrm{C}$ under an atmosphere of argon. The anion was allowed to form over ca. 30 minutes, resulting in a light orange homogeneous solution. In another flask, $[\mathrm{RhCl}(\mathrm{COD})]_{2}(6.2 \mathrm{mg}, 0.013 \mathrm{mmol})$ and tris(2,2,2-trifluoroethyl) phosphite $(0.011 \mathrm{~mL}, 0.050$ mmol) were dissolved in anhydrous tetrahydrofuran $(1.5 \mathrm{~mL})$ at room temperature under an atmosphere of argon and the mixture stirred for $c a .5$ minutes resulting in a light yellow homogeneous solution. All three flasks were then cooled to $-10{ }^{\circ} \mathrm{C}$, and the cyanohydrin solution was added to the carbonate solution, followed quickly by addition of the catalyst solution via Teflon $^{\circledR}$ cannula. The resulting mixture was allowed to stir for $c a$. 16 hours before being cooled to $40{ }^{\circ} \mathrm{C}$ (dry ice/acetonitrile bath). Tetrabutylammonium fluoride $(3.00 \mathrm{~mL}, 3.00 \mathrm{mmol}$; $1 \mathrm{M}$ solution in tetrahydrofuran) was then added dropwise and the mixture stirred for an additional 1 hour at this temperature. The reaction mixture was quenched with saturated aqueous ammonium chloride solution and allowed to warm to room temperature before being partitioned between diethyl ether and saturated aqueous ammonium chloride solution. The combined organic layers were dried (anhyd. $\mathrm{MgSO}_{4}$ ), filtered and concentrated in vacuo to afford the crude product. Purification by flash column chromatography (silica gel, eluting with 3-9\% diethyl ether/hexane) afforded the ketone $6 \mathbf{b}$ (126.2 $\mathrm{mg}, 87 \%$ ) as a pale yellow oil.

\section{Spectral Data for the Enantiomerically Enriched Ketone Products 6a-c}

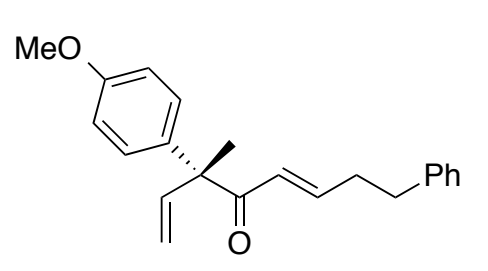

(R,E)-3-(4-Methoxyphenyl)-3-methyl-8-phenylocta-1,5-dien-4-one

Color and State: Colorless oil; $b: l \geq 19: 1$

$[\alpha]_{\mathrm{D}}^{20}+30.4\left(c=1.0, \mathrm{CHCl}_{3}\right)$; Chiral HPLC analysis (CHIRALCEL

OD column), 85:15 hexane/isopropanol at $1.0 \mathrm{~mL} / \mathrm{min}$ flow rate; $t_{\mathrm{R}}(R)$-enantiomer (major) $=15.3$ min., $t_{\mathrm{R}}(S)$-enantiomer (minor) $=18.3 \mathrm{~min}$; 91\% ee; $92 \%$ cee.

${ }^{1}$ H NMR $\left(500 \mathrm{MHz}, \mathrm{CDCl}_{3}\right) \delta$ 7.25-7.23 (m, 2H), 7.19-7.16 (m, 1H), 7.10-7.06 (m, 4H), 6.94 (dt, $J$ $=15.2,7.0 \mathrm{~Hz}, 1 \mathrm{H}), 6.87-6.84(\mathrm{~m}, 2 \mathrm{H}), 6.33(\mathrm{dd}, J=17.4,10.7 \mathrm{~Hz}, 1 \mathrm{H}), 6.01(\mathrm{dt}, J=15.4,1.4 \mathrm{~Hz}$, 1H), $5.27(\mathrm{dd}, J=10.7,0.7 \mathrm{~Hz}, 1 \mathrm{H}), 5.06(\mathrm{dd}, J=17.5,0.7 \mathrm{~Hz}, 1 \mathrm{H}), 3.81(\mathrm{~s}, 3 \mathrm{H}), 2.68(\mathrm{t}, J=7.6 \mathrm{~Hz}$, 2H), 2.41 (app. qd, $J=7.4,1.3 \mathrm{~Hz}, 2 \mathrm{H}), 1.50$ (s, 3H). 
${ }^{13}$ C NMR (125 MHz, $\mathrm{CDCl}_{3}$ ) $\delta 198.97$ (e), 158.59 (e), 145.94 (o), 140.92 (e), 140.46 (o), 134.95 (e), 128.49 (o), 128.46 (o), 128.40 (o), 127.33 (o), 126.14 (o), 116.16 (e), 114.21 (o), 57.73 (e), 55.34 (o), 34.45 (e), 34.09 (e), 23.07 (o).

IR (Neat) 3086 (w), 3060 (w), 3028 (w), 2981 (w), 2932 (w), 2857 (w), 1692 (s), 1626 (m), 1610 (m), 1511 (s), 1455 (m), 1251 (vs), 1033 (m), 924 (w), 701 (m) $\mathrm{cm}^{-1}$.

HRMS (EI, $\mathrm{M}^{+}$) calcd for $\mathrm{C}_{22} \mathrm{H}_{24} \mathrm{O}_{2} 320.1776$, found 320.1781 .

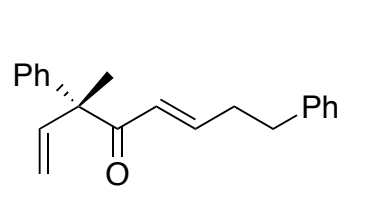

\section{$(R, E)-3-M e t h y l-3,8-d i p h e n y l o c t a-1,5-d i e n-4-o n e ~(6 b)$}

Color and State: Pale yellow oil; $b: l \geq 19: 1$

$[\alpha]_{\mathrm{D}}^{20}+42.4\left(c=1.0, \mathrm{CHCl}_{3}\right)$; Chiral HPLC analysis (CHIRALCEL OJ-H column), 90:10 hexane/isopropanol at $1.5 \mathrm{~mL} / \mathrm{min}$ flow rate; $t_{\mathrm{R}}(S)$-enantiomer (minor) $=12.3 \mathrm{~min}$., $t_{\mathrm{R}}(R)$-enantiomer (major) $=19.1 \mathrm{~min}$; 96\% ee; 96\% cee.

${ }^{1}$ H NMR $\left(500 \mathrm{MHz}, \mathrm{CDCl}_{3}\right) \delta$ 7.34-7.31 (m, 2H), 7.27-7.23 (m, 3H), 7.19-7.16 (m, 3H), 7.09-7.08 (m, 2H), $6.95(\mathrm{dt}, J=15.2,7.1 \mathrm{~Hz}, 1 \mathrm{H}), 6.35(\mathrm{dd}, J=17.4,10.8 \mathrm{~Hz}, 1 \mathrm{H}), 6.00(\mathrm{dt}, J=15.4,1.3 \mathrm{~Hz}$, $1 \mathrm{H}), 5.30(\mathrm{~d}, J=10.7 \mathrm{~Hz}, 1 \mathrm{H}), 5.09$ (d, $J=17.5 \mathrm{~Hz}, 1 \mathrm{H}), 2.67$ (t, $J=7.6 \mathrm{~Hz}, 2 \mathrm{H}), 2.40$ (app. qd, $J=$ 7.4, $1.2 \mathrm{~Hz}, 2 \mathrm{H}), 1.52(\mathrm{~s}, 3 \mathrm{H})$.

${ }^{13}$ C NMR (125 MHz, $\left.\mathrm{CDCl}_{3}\right) \delta 198.76$ (e), 146.14 (o), 143.11 (e), 140.91 (e), 140.11 (o), 128.85 (o), 128.51 (o), 128.47 (o), 127.42 (o), 127.31 (o), 127.04 (o), 126.16 (o), 116.51 (e), 58.48 (e), 34.45 (e), 34.11 (e), 23.05 (o).

IR (Neat) 3061 (w), 3026 (w), 2982 (w), 2934 (w), 2858 (w), 1693 (m), 1625 (m), 1603 (w), 1493 (m), $1446(\mathrm{w}), 1264(\mathrm{w}), 1075(\mathrm{~m}), 923(\mathrm{~m}), 698(\mathrm{~s}) \mathrm{cm}^{-1}$.

HRMS (ESI, $[\mathrm{M}+\mathrm{H}]^{+}$) calcd for $\mathrm{C}_{21} \mathrm{H}_{23} \mathrm{O} 291.1743$, found 291.1738.

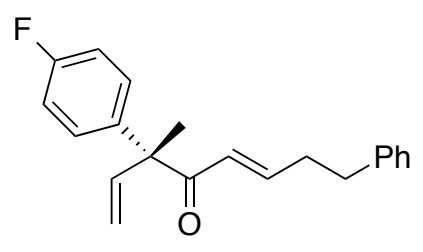

\section{(R,E)-3-(4-Fluorophenyl)-3-methyl-8-phenylocta-1,5-dien-4-one (6c)}

Color and State: Colorless oil; $b: l \geq 19: 1$

$[\alpha]_{\mathrm{D}}^{20}+51.6\left(c=1.0, \mathrm{CHCl}_{3}\right)$; Chiral HPLC analysis (CHIRALCEL OJ-H column), 99:1 hexane/isopropanol at $1.0 \mathrm{~mL} / \mathrm{min}$ flow rate; $t_{\mathrm{R}}(S)$ enantiomer $($ minor $)=30.2 \mathrm{~min}$., $t_{\mathrm{R}}(R)$-enantiomer $($ major $)=37.0 \mathrm{~min}$; $92 \%$ ee; $92 \%$ cee .

${ }^{1}$ H NMR $\left(500 \mathrm{MHz}, \mathrm{CDCl}_{3}\right) \delta$ 7.25-7.22 (m, 2H), 7.19-7.16 (m, 1H), 7.13-7.08 (m, 4H), 7.03-6.99 $(\mathrm{m}, 2 \mathrm{H}), 6.95(\mathrm{dt}, J=15.3,6.9 \mathrm{~Hz}, 1 \mathrm{H}), 6.31(\mathrm{dd}, J=17.4,10.8 \mathrm{~Hz}, 1 \mathrm{H}), 5.97(\mathrm{dt}, J=15.3,1.5 \mathrm{~Hz}$, 
1H), $5.30(\mathrm{dd}, J=10.8,0.7 \mathrm{~Hz}, 1 \mathrm{H}), 5.07(\mathrm{dd}, J=17.4,0.7 \mathrm{~Hz}, 1 \mathrm{H}), 2.69$ (t, $J=7.6 \mathrm{~Hz}, 2 \mathrm{H}), 2.44-$ $2.40(\mathrm{~m}, 2 \mathrm{H}), 1.50(\mathrm{~s}, 3 \mathrm{H})$.

${ }^{13}$ C NMR (125 MHz, $\left.\mathrm{CDCl}_{3}\right) \delta 198.47$ (e), 161.87 (e, d, $\left.{ }^{1} J_{C-F}=246.3 \mathrm{~Hz}\right), 146.52$ (o), 140.79 (e), 140.00 (o), 138.76 (e, d, $\left.{ }^{4} J_{C-F}=3.2 \mathrm{~Hz}\right), 128.94$ (o, d, $\left.{ }^{3} J_{C-F}=7.8 \mathrm{~Hz}\right), 128.51$ (o), 128.45 (o), 127.15 (o), 126.18 (o), 116.66 (e), 115.68 (o, d, ${ }^{2} J_{C-F}=21.2 \mathrm{~Hz}$ ), 57.90 (e), 34.37 (e), 34.07 (e), 23.16 (o).

IR (Neat) 3087 (w), 3063 (w), 3027 (w), 2981 (w), 2931 (w), 2856 (w), 1693 (s), 1625 (s), 1604 (m), 1508 (s), 1454 (m), 1225 (s), 1163 (m), 924 (m), 699 (vs) $\mathrm{cm}^{-1}$.

HRMS (EI, $\mathrm{M}^{+}$) calcd for $\mathrm{C}_{21} \mathrm{H}_{21} \mathrm{FO} 308.1576$, found 308.1572 .

\section{Preparation of $\alpha, \alpha^{\prime}$-Dialkyl Ketone 9}

The tertiary allylic carbonate 7 was prepared from the commercially available $(R)$-linalool $(99: 1$ er) according to the previously published procedure. ${ }^{1 \mathrm{~b}}$

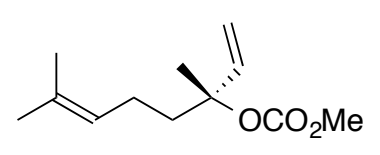

(R)-3,7-Dimethylocta-1,6-dien-3-yl methyl carbonate (7)

Color and State: Colorless oil.

All spectral data matched the published values. ${ }^{3 \mathrm{c}}$

$[\alpha]_{\mathrm{D}}^{20}-1.1\left(c=1.0, \mathrm{CHCl}_{3}\right),\left[\right.$ lit. $\left.^{3 \mathrm{c}}[\alpha]_{\mathrm{D}}^{20}-3.2\left(c=1.0, \mathrm{CHCl}_{3}\right)\right]$

${ }^{1}$ H NMR $\left(500 \mathrm{MHz}, \mathrm{CDCl}_{3}\right) \delta 6.00(\mathrm{dd}, J=17.6,11.0 \mathrm{~Hz}, 1 \mathrm{H}), 5.21(\mathrm{~d}, J=17.8 \mathrm{~Hz}, 1 \mathrm{H}), 5.18(\mathrm{~d}, J$ $=11.5 \mathrm{~Hz}, 1 \mathrm{H}), 5.10-5.06(\mathrm{~m}, 1 \mathrm{H}), 3.72(\mathrm{~s}, 3 \mathrm{H}), 2.00$ (app. q, $J=7.9 \mathrm{~Hz}, 2 \mathrm{H}), 1.90-1.77(\mathrm{~m}, 2 \mathrm{H})$, $1.67(\mathrm{~s}, 3 \mathrm{H}), 1.59(\mathrm{~s}, 3 \mathrm{H}), 1.57(\mathrm{~s}, 3 \mathrm{H})$.

IR (Neat) 2970 (w), 2920 (w), 2858 (w), 1746 (s), 1645 (w), 1440 (m), 1266 (vs), 1082 (m), 993 (w), $793(\mathrm{w}) \mathrm{cm}^{-1}$.

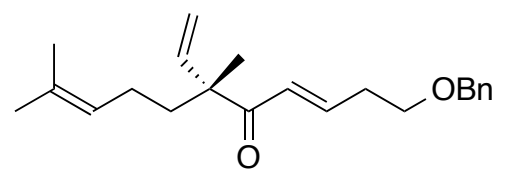

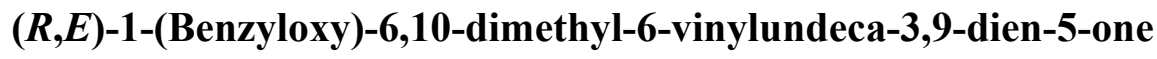
(8). Lithium chloride (64.0 $\mathrm{mg}, 1.5 \mathrm{mmol})$ was suspended in anhydrous tetrahydrofuran $(3 \mathrm{~mL})$ and the mixture was stirred at -10 ${ }^{\circ} \mathrm{C}$ under an atmosphere of argon for $c a .15$ minutes. (E)-6-(benzyloxy)-2-(tertbutyldimethylsilyloxy)hex-3-enenitrile $2 \mathrm{r}(332.0 \mathrm{mg}, 1.0 \mathrm{mmol})$ was added followed by the dropwise addition of lithium bis(trimethylsilyl)amine $(1.40 \mathrm{~mL}, 1.40 \mathrm{mmol}$; $1 \mathrm{M}$ solution in tetrahydrofuran) and the anion allowed to form over $c a .30$ minutes, resulting in a light orange homogenous solution. In a separate flask, $[\mathrm{RhCl}(\mathrm{COD})] 2(6.20 \mathrm{mg}, 0.013 \mathrm{mmol})$ and tris(2,2,2trifluoroethyl) phosphite $(0.011 \mathrm{~mL}, 0.050 \mathrm{mmol})$ were dissolved in anhydrous tetrahydrofuran $(2$ 
$\mathrm{mL}$ ) at room temperature under an atmosphere of argon and the mixture stirred for $c a .5$ minutes resulting in a light yellow homogeneous solution, which was cooled to $-10{ }^{\circ} \mathrm{C}$. The catalyst solution was then added to the anion via Teflon ${ }^{\circledR}$ cannula, followed immediately by the addition of $(R)-3,7$ dimethylocta-1,6-dien-3-yl methyl carbonate $7(106.0 \mathrm{mg}, 0.50 \mathrm{mmol})$ via tared $500 \mu \mathrm{L}$ gastight syringe. The resulting mixture was allowed to stir for $c a$. 16 hours before being cooled to $-40{ }^{\circ} \mathrm{C}$ (dry ice/acetonitrile bath). Tetrabutylammonium fluoride $(4.00 \mathrm{~mL}, 4.00 \mathrm{mmol}$; $1 \mathrm{M}$ solution in tetrahydrofuran) was then added dropwise and the mixture stirred for an additional 1 hour at this temperature. The reaction mixture was quenched with saturated aqueous ammonium chloride solution and allowed to warm to room temperature before being partitioned between diethyl ether and saturated aqueous ammonium chloride solution. The combined organic layers were dried (anhyd. $\mathrm{MgSO}_{4}$ ), filtered and concentrated in vacuo to afford the crude product. Purification by flash column chromatography (silica gel, eluting with 10-20\% diethyl ether/hexane) afforded the ketone 8 $(134.4 \mathrm{mg}, 82 \%)$ as a colorless oil: $[\alpha]_{\mathrm{D}}^{20}-10.1\left(c=1.0, \mathrm{CHCl}_{3}\right)$; Chiral HPLC analysis (CHIRALCEL OJ-H column), 99:0.7:0.3 hexane/isopropanol/methanol at $0.7 \mathrm{~mL} / \mathrm{min}$ flow rate; $t_{\mathrm{R}}$ $(S)$-enantiomer $($ minor $)=20.9$ min., $t_{\mathrm{R}}(R)$-enantiomer $($ major $)=23.1 \mathrm{~min}$; $92 \%$ ee; 94\% cee.

${ }^{1} \mathbf{H}$ NMR $\left(500 \mathrm{MHz}, \mathrm{CDCl}_{3}\right) \delta$ 7.36-7.26 (m, 5H), $6.93(\mathrm{dt}, J=15.4,7.0 \mathrm{~Hz}, 1 \mathrm{H}), 6.50(\mathrm{dt}, J=15.3$, $1.5 \mathrm{~Hz}, 1 \mathrm{H}), 5.91(\mathrm{dd}, J=17.6,10.8 \mathrm{~Hz}, 1 \mathrm{H}), 5.19$ (dd, $J=10.7,0.9 \mathrm{~Hz}, 1 \mathrm{H}), 5.13$ (dd, $J=17.5,0.8$ $\mathrm{Hz}, 1 \mathrm{H}), 5.07$ (app. t pentet, $J=7.1,1.4 \mathrm{~Hz}, 1 \mathrm{H}), 4.51(\mathrm{~s}, 2 \mathrm{H}), 3.57$ (t, $J=6.6 \mathrm{~Hz}, 2 \mathrm{H}), 2.50$ (app. qd, $J=6.7,1.5 \mathrm{~Hz}, 2 \mathrm{H}), 1.94-1.86(\mathrm{~m}, 1 \mathrm{H}), 1.84-1.76(\mathrm{~m}, 1 \mathrm{H}), 1.71\left(\mathrm{ddd}, \mathrm{A}\right.$ of ABXY, $J_{A B}=13.6 \mathrm{~Hz}$, $\left.J_{A X}=12.0 \mathrm{~Hz}, J_{A Y}=5.0 \mathrm{~Hz}, 1 \mathrm{H}\right), 1.66(\mathrm{~s}, 3 \mathrm{H}), 1.60\left(\mathrm{ddd}, \mathrm{B}\right.$ of ABXY, $J_{A B}=13.6 \mathrm{~Hz}, J_{B X}=12.0 \mathrm{~Hz}$, $\left.J_{B Y}=5.1 \mathrm{~Hz}, 1 \mathrm{H}\right), 1.55(\mathrm{~s}, 3 \mathrm{H}), 1.22(\mathrm{~s}, 3 \mathrm{H})$.

${ }^{13}$ C NMR (125 MHz, $\mathrm{CDCl}_{3}$ ) $\delta 200.86$ (e), 143.58 (o), 141.62 (o), 138.26 (e), 131.95 (e), 128.51 (o), 127.75 (o), 126.92 (o), 124.23 (o), 115.35 (e), 73.16 (e), 68.60 (e), 53.08 (e), 37.25 (e), 32.99 (e), 25.76 (o), 23.07 (e), 19.96 (o), 17.70 (o).

IR (Neat) 3084 (w), 3064 (w), 3030 (w), 2969 (w), 2927 (m), 2856 (m), 1691 (s), 1625 (s), 1496 (w), 1454 (m), 1099 (s), 919 (m), 698 (vs) $\mathrm{cm}^{-1}$.

HRMS (ESI, [M+H] $]^{+}$) calcd for $\mathrm{C}_{22} \mathrm{H}_{31} \mathrm{O}_{2} 327.2319$, found 327.2311.

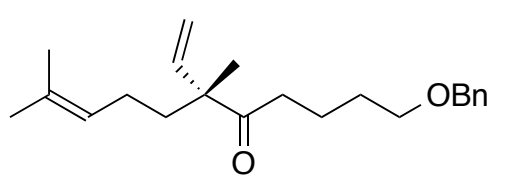

(R)-1-(Benzyloxy)-6,10-dimethyl-6-vinylundeca-9-en-5-one

$(R, E)$-1-(benzyloxy)-6,10-dimethyl-6-vinylundeca-3,9-dien-5-one $\mathbf{8}$ 
(111.0 $\mathrm{mg}, 0.340 \mathrm{mmol}$ ) was added to a stirring suspension of (triphenylphosphine)copper hydride hexamer $(274 \mathrm{mg}, 0.126 \mathrm{mmol})$ and water $(0.025 \mathrm{~mL}, 1.36 \mathrm{mmol})$ in benzene $(8.5 \mathrm{~mL})$ at room temperature under an atmosphere of argon. The resulting solution was allowed to stir for $c a .2$ hours under argon followed by 1 hour under air before being filtered through celite, washed with diethyl ether and concentrated in vacuo to afford the crude product. Purification by flash column chromatography (silica gel, eluting with $10-14 \%$ diethyl ether/hexane) afforded the $\alpha, \alpha^{\prime}$-dialkyl ketone 9 (105.1 mg, $94 \%)$ as a colorless oil: $[\alpha]_{\mathrm{D}}^{20}-10.7\left(c=1.0, \mathrm{CHCl}_{3}\right)$.

${ }^{1} \mathbf{H}$ NMR $\left(500 \mathrm{MHz}, \mathrm{CDCl}_{3}\right) \delta$ 7.35-7.32 (m, 4H), 7.29-7.27 (m, 1H), $5.89(\mathrm{dd}, J=17.5,10.8 \mathrm{~Hz}$, 1H), 5.17 (d, $J=10.7 \mathrm{~Hz}, 1 \mathrm{H}), 5.11$ (d, $J=17.6 \mathrm{~Hz}, 1 \mathrm{H}), 5.07$ (app. tt, $J=7.1,1.3 \mathrm{~Hz}, 1 \mathrm{H}$ ), 4.49 (s, $2 \mathrm{H}), 3.46(\mathrm{t}, J=6.1 \mathrm{~Hz}, 2 \mathrm{H}), 2.46$ (app. qt, $J=17.5,7.0 \mathrm{~Hz}, 2 \mathrm{H}), 1.92-1.84(\mathrm{~m}, 1 \mathrm{H}), 1.83-1.75(\mathrm{~m}$, $1 \mathrm{H}), 1.71-1.20(\mathrm{~m}, 6 \mathrm{H}), 1.67$ (s, 3H), 1.57 (s, 3H), $1.20(\mathrm{~s}, 3 \mathrm{H})$.

${ }^{13}$ C NMR (125 MHz, CDCl $) \delta 212.65$ (e), 141.79 (o), 138.70 (e), 132.00 (e), 128.47 (o), 127.75 (o), 127.62 (o), 124.15 (o), 115.07 (e), 73.03 (e), 70.29 (e), 54.34 (e), 37.63 (e), 37.57 (e), 29.37 (e), 25.79 (o), 23.15 (e), 20.85 (e), 19.67 (o), 17.74 (o).

IR (Neat) 3089 (w), 3066 (w), 3033 (w), 2929 (m), 2858 (m), 1706 (s), 1633 (w), 1496 (w), 1454 (m), 1102 (s), $918(\mathrm{~m}), 697$ (vs) $\mathrm{cm}^{-1}$.

HRMS (EI, $\mathrm{M}^{+}$) calcd for $\mathrm{C}_{22} \mathrm{H}_{32} \mathrm{O}_{2} 328.2402$, found 328.2409 .

\section{Proof of Configuration/Preparation of LY426965}

(S)-2-Phenylbut-3-en-2-ol (ent-5b) ${ }^{3}$

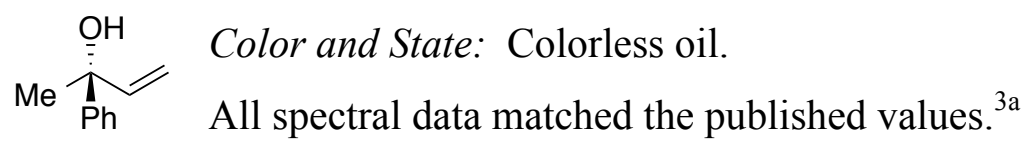

$[\alpha]_{\mathrm{D}}^{20}-22.8\left(c=1.0, \mathrm{CHCl}_{3}\right)$; Chiral HPLC analysis (CHIRALCEL OJ-H column), 98:2 hexane/isopropanol at $1.0 \mathrm{~mL} / \mathrm{min}$ flow rate; $t_{\mathrm{R}}(S)$-enantiomer (major) $=22.0 \mathrm{~min}$., $t_{\mathrm{R}}(R)$ enantiomer (minor) $=32.1$ min.; $96 \%$ ee .

${ }^{1}$ H NMR $\left(500 \mathrm{MHz}, \mathrm{CDCl}_{3}\right) \delta$ 7.49-7.47 (m, 2H), 7.37-7.33 (m, 2H), 7.28-7.24 (m, 1H), 6.18 (dd, $J$ $=17.3,10.6 \mathrm{~Hz}, 1 \mathrm{H}), 5.31(\mathrm{dd}, J=17.3,1.0 \mathrm{~Hz}, 1 \mathrm{H}), 5.15(\mathrm{dd}, J=10.7,1.1 \mathrm{~Hz}, 1 \mathrm{H}), 1.89$ (br. s, $1 \mathrm{H}), 1.66(\mathrm{~s}, 3 \mathrm{H})$.

IR (Neat) 3390 (br, w), 3086 (w), 3060 (w), 3026 (w), 2980 (w), 2933 (w), 2878 (w), 1637 (w), 1601 (w), 921 (s), 765 (s), 698 (vs) $\mathrm{cm}^{-1}$. 


\section{(S)-1-Cyclohexenyl-2-methyl-2-phenylbut-3-en-1-one}

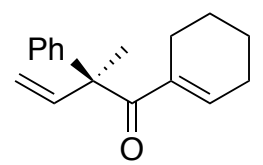

Prepared according to the general procedure for the stereospecific reaction.

$[\alpha]_{\mathrm{D}}^{20}-52.1\left(c=1.0, \mathrm{CHCl}_{3}\right)$; Chiral HPLC analysis (CHIRALCEL OJ-H column),

95:5 hexane/isopropanol at $1.0 \mathrm{~mL} / \mathrm{min}$ flow rate; $t_{\mathrm{R}}(S)$-enantiomer (major) $=8.0 \mathrm{~min}$., $t_{\mathrm{R}}(R)$ enantiomer $($ minor $)=16.7$ min.; 92\% ee.

${ }^{1}$ H NMR $\left(500 \mathrm{MHz}, \mathrm{CDCl}_{3}\right) \delta$ 7.32-7.29 (m, 2H), 7.24-7.20 (m, 1H), 7.18-7.16 (m, 2H), 6.55 (dd, $J$ $=17.4,10.7 \mathrm{~Hz}, 1 \mathrm{H}), 6.20-6.18(\mathrm{~m}, 1 \mathrm{H}), 5.27(\mathrm{dd}, J=10.8,0.8 \mathrm{~Hz}, 1 \mathrm{H}), 5.10(\mathrm{dd}, J=17.4,0.7 \mathrm{~Hz}$, $1 \mathrm{H}), 2.23-2.17(\mathrm{~m}, 1 \mathrm{H}), 2.07-1.90(\mathrm{~m}, 3 \mathrm{H}), 1.58-1.51(\mathrm{~m}, 2 \mathrm{H}), 1.55(\mathrm{~s}, 3 \mathrm{H}), 1.49-1.44(\mathrm{~m}, 2 \mathrm{H})$.

${ }^{13}$ C NMR (125 MHz, CDCl $) \delta 202.32$ (e), 145.58 (e), 141.80 (o), 141.32 (o), 136.72 (e), 128.77 (o), 126.60 (o), 126.27 (o), 115.84 (e), 58.31 (e), 26.44 (o), 25.94 (e), 24.96 (e), 22.24 (e), 21.55 (e).

IR (Neat) 3084 (w), 3060 (w), 3024 (w), 2984 (w), 2931 (m), 2860 (w), 1664 (m), 1636 (m), 1492 (w), 1447 (m), 1221 (m), 1075 (w), $919(\mathrm{~m}), 700$ (vs) $\mathrm{cm}^{-1}$.

HRMS (EI, $\mathrm{M}^{+}$) calcd for $\mathrm{C}_{17} \mathrm{H}_{20} \mathrm{O} 240.1514$, found 210.1511 .

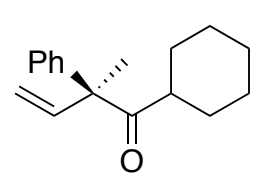

(S)-1-Cyclohexyl-2-methyl-2-phenylbut-3-en-1-one. $\quad$ L-Selectride $^{\circledR}(2.76 \mathrm{~mL}$, $2.76 \mathrm{mmol} ; 1 \mathrm{M}$ solution in tetrahydrofuran) was added dropwise to a stirring solution of (S)-1-cyclohexenyl-2-methyl-2-phenylbut-3-en-1-one (332 mg, 1.38 $\mathrm{mmol})$ in tetrahydrofuran $(13.8 \mathrm{~mL})$ at $0{ }^{\circ} \mathrm{C}$ under an atmosphere of argon. The solution was allowed to slowly warm to room temperature and stirred for $c a .5$ hours. The mixture was then cooled to $0{ }^{\circ} \mathrm{C}$ and quenched by the addition of a saturated aqueous ammonium chloride solution before being partitioned between diethyl ether and saturated aqueous ammonium chloride solution. The combined organic layers were dried (anhyd. $\mathrm{MgSO}_{4}$ ), filtered and concentrated in vacuo to afford the crude product. Purification by flash column chromatography (silica gel, eluting with $2-6 \%$ diethyl ether/hexane) afforded the cyclohexyl ketone $(276 \mathrm{mg}, 83 \%)$ as a colorless oil: $[\alpha]_{\mathrm{D}}^{20}-14.3(c=1.0$, $\mathrm{CHCl}_{3}$ ).

${ }^{1}$ H NMR $\left(500 \mathrm{MHz}, \mathrm{CDCl}_{3}\right) \delta$ 7.35-7.32 (m, 2H), 7.28-7.25 (m, 1H), 7.22-7.20 (m, 2H), $6.52(\mathrm{dd}, J$ $=17.4,10.8 \mathrm{~Hz}, 1 \mathrm{H}), 5.34(\mathrm{~d}, J=10.8 \mathrm{~Hz}, 1 \mathrm{H}), 5.14(\mathrm{~d}, J=17.4 \mathrm{~Hz}, 1 \mathrm{H}), 2.45\left(\mathrm{tt}, \mathrm{A}\right.$ of $\mathrm{AM}_{2} \mathrm{X}_{2}, J_{A M}$ $\left.=11.1 \mathrm{~Hz}, J_{A X}=3.8 \mathrm{~Hz}, 1 \mathrm{H}\right), 1.66-1.54(\mathrm{~m}, 4 \mathrm{H}), 1.53(\mathrm{~s}, 3 \mathrm{H}), 1.44-1.31(\mathrm{~m}, 3 \mathrm{H}), 1.21-0.97(\mathrm{~m}, 3 \mathrm{H})$.

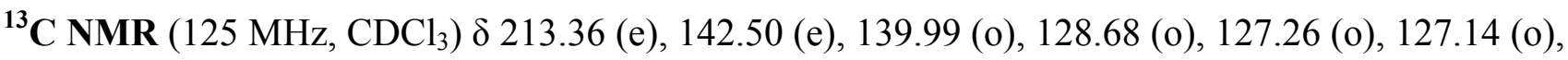
116.45 (e), 60.09 (e), 47.70 (o), 31.23 (e), 30.64 (e), 25.77 (e), 25.66 (e), 22.39 (o). 
IR (Neat) 3088 (w), 3060 (w), 3023 (w), 2930 (m), 2854 (m), 1703 (s), 1633 (w), 1600 (w), 1492 (w), 1447 (m), 1244 (w), 1148 (w), 1065 (m), 921 (m), 701 (vs) $\mathrm{cm}^{-1}$.

HRMS (EI, $\mathrm{M}^{+}$) calcd for $\mathrm{C}_{17} \mathrm{H}_{22} \mathrm{O} 242.1671$, found 242.1668 .

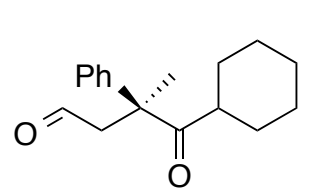

(S)-4-Cyclohexyl-3-methyl-4-oxo-3-phenylbutanal. A solution of $(S)-1-$ cyclohexyl-2-methyl-2-phenylbut-3-en-1-one (260 $\mathrm{mg}, 1.07 \mathrm{mmol})$ in anhydrous diethyl ether $(1.1 \mathrm{~mL})$ was added dropwise to a stirring suspension of lithium aluminium hydride $(81.0 \mathrm{mg}, 2.15 \mathrm{mmol})$ in anhydrous diethyl ether $(4.3 \mathrm{~mL})$ at $0{ }^{\circ} \mathrm{C}$ under an atmosphere of argon. The resulting mixture was then allowed to warm slowly to room temperature and stirred until completion (tlc control). Upon completion, the mixture was cooled to $0{ }^{\circ} \mathrm{C}$ and quenched by the addition water $(0.2 \mathrm{~mL})$ followed by $1 \mathrm{M}$ aqueous sodium hydroxide solution $(0.2$ $\mathrm{mL}$ ) before being warmed to room temperature. The mixture was then filtered through celite, dried (anhyd. $\mathrm{MgSO}_{4}$ ), filtered and concentrated in vacuo to afford the crude alcohol (233 $\mathrm{mg}, 89 \%$ ) as a colorless oil. The crude alcohol ( $233 \mathrm{mg}, 0.95 \mathrm{mmol}$ ) was then added to a stirring solution of 9borabicyclo[3.3.1]nonane dimer $(345 \mathrm{mg}, 1.43 \mathrm{mmol})$ in anhydrous tetrahydrofuran $(9.5 \mathrm{~mL})$ at room temperature under an atmosphere of argon. A reflux condenser was then fitted to the flask and the mixture heated to $70{ }^{\circ} \mathrm{C}$ for $\mathrm{ca}$. 16 hours. Upon completion, the solution was then cooled to $0{ }^{\circ} \mathrm{C}$ and water $(4 \mathrm{~mL})$, sodium hydroxide $(342 \mathrm{mg}, 8.55 \mathrm{mmol}$ ) and sodium perborate tetrahydrate (699 $\mathrm{mg}, 8.55 \mathrm{mmol}$ ) were added sequentially and the mixture was allowed to warm to room temperature before being heated to $50{ }^{\circ} \mathrm{C}$ for $c a .4$ hours. After cooling to room temperature the mixture was partitioned between diethyl ether and water. The combined organic layers were dried (anhyd. $\mathrm{MgSO}_{4}$ ), filtered and concentrated in vacuo to afford the crude diol, which was used directly in the next step without further purification.

Dimethyl sulfoxide $(0.24 \mathrm{~mL}, 3.34 \mathrm{mmol})$ was added dropwise to a stirred solution of oxalyl dichloride $(0.15 \mathrm{~mL}, 1.68 \mathrm{mmol})$ in anhydrous dichloromethane $(8.0 \mathrm{~mL})$ at $-78{ }^{\circ} \mathrm{C}$ under an atmosphere of argon. The mixture was then allowed to stir for $c a$. 30 minutes before a solution of the crude diol in anhydrous dichloromethane $(2.0 \mathrm{~mL})$ was added dropwise. After an additional 30 minutes, triethylamine $(1.0 \mathrm{~mL}, 7.60 \mathrm{mmol})$ was added and the mixture was stirred for $c a .15$ minutes at $-78{ }^{\circ} \mathrm{C}$ before being warmed to room temperature. The reaction mixture was quenched by the addition of water $(5 \mathrm{~mL})$ and partitioned between dichloromethane and water. The combined organic layers were dried (anhyd. $\mathrm{MgSO}_{4}$ ), filtered and concentrated in vacuo to afford the crude 
product. Purification by flash column chromatography (silica gel, eluting with $15-25 \%$ diethyl ether/hexane) afforded the keto-aldehyde (162 mg, 77\% over two steps) as a colorless oil: $[\alpha]_{\mathrm{D}}^{20}$ $+120.7\left(c=1.0, \mathrm{CHCl}_{3}\right),\left[\mathrm{lit}^{4}[\alpha]_{\mathrm{D}}^{20}+158.6\left(c=1.0, \mathrm{CHCl}_{3}\right)\right]$.

${ }^{1} \mathbf{H}$ NMR $\left(500 \mathrm{MHz}, \mathrm{CDCl}_{3}\right) \delta 9.59(\mathrm{t}, J=2.4 \mathrm{~Hz}, 1 \mathrm{H}), 7.39-7.36(\mathrm{~m}, 2 \mathrm{H}), 7.32-7.27(\mathrm{~m}, 3 \mathrm{H}), 2.96$ (dd, A of ABX, $\left.J_{A B}=16.1 \mathrm{~Hz}, J_{A X}=2.6 \mathrm{~Hz}, 1 \mathrm{H}\right), 2.77\left(\mathrm{dd}, \mathrm{B}\right.$ of ABX, $J_{A B}=16.1 \mathrm{~Hz}, J_{B X}=2.1 \mathrm{~Hz}$, $1 \mathrm{H}), 2.40\left(\mathrm{tt}, \mathrm{A}\right.$ of $\left.\mathrm{AM}_{2} \mathrm{X}_{2}, J_{A M}=11.6 \mathrm{~Hz}, J_{A X}=3.3 \mathrm{~Hz}, 1 \mathrm{H}\right), 1.78-1.65(\mathrm{~m}, 2 \mathrm{H}), 1.75(\mathrm{~s}, 3 \mathrm{H}), 1.58-$ 1.54 (m, 2H), 1.37 (app. qd, $J=12.5,3.3 \mathrm{~Hz}, 2 \mathrm{H}$ ), 1.18-1.08 (m, 3H), 0.93 (app. qt, $J=12.9,3.5 \mathrm{~Hz}$, $1 \mathrm{H})$.

${ }^{13}$ C NMR (125 MHz, CDCl $)$ ) 213.94 (e), 201.70 (o), 140.04 (e), 129.05 (o), 127.75 (o), 126.68 (o), 54.54 (e), 52.16 (e), 46.21 (o), 30.83 (e), 30.68 (e), 25.67 (e), 25.63 (e), 25.54 (e), 20.39 (o).

IR (Neat) 2932 (s), 2855 (m), 2738 (w), 1720 (s), 1701 (vs), 1600 (w), 1496 (w), 1447 (m), 1244 (w), $1144(\mathrm{w}), 991(\mathrm{~m}), 702(\mathrm{~m}) \mathrm{cm}^{-1}$.

HRMS (ESI, $[\mathrm{M}+\mathrm{H}]^{+}$) calcd for $\mathrm{C}_{17} \mathrm{H}_{23} \mathrm{O}_{2} 259.1693$, found 259.1682 .

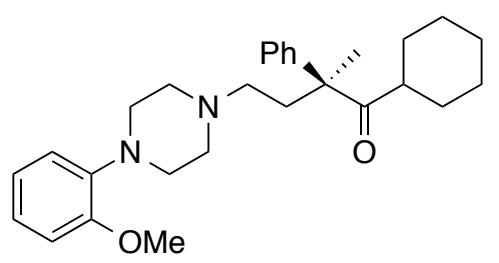

(S)-1-Cyclohexyl-4-(4-(2-methoxyphenyl)piperazin-1-yl)-2-methyl2-phenylbutan-1-one (LY426965). Prepared according to a previously reported procedure. $^{4}$ A solution of 1-(2methoxyphenyl)piperazine (101 $\mathrm{mg}, 0.525 \mathrm{mmol}$ ) in anhydrous dichloroethane was added to a stirring solution (S)-4-cyclohexyl-3-methyl-4-oxo-3-phenylbutanal (129 $\mathrm{mg}, 0.5 \mathrm{mmol})$ in anhydrous dichloroethane $(1.5 \mathrm{~mL})$ at room temperature under an atmosphere of argon. Sodium triacetoxyborohydride $(212 \mathrm{mg}, 1.0 \mathrm{mmol})$ was then added and the resulting mixture allowed to stir for $c a$. 6 hours. The reaction mixture was quenched by the addition of a saturated aqueous sodium bicarbonate solution and extracted into dichloromethane. The combined organic layers were dried (anhyd. $\mathrm{MgSO}_{4}$ ), filtered and concentrated in vacuo to afford the crude product. Purification by flash column chromatography (silica gel, eluting with $40-60 \%$ ethyl acetate/hexane) afforded the piperazine $(175 \mathrm{mg}, 81 \%)$ as a colorless oil: $[\alpha]_{\mathrm{D}}^{20}+33.8(c=0.95$, $\left.\mathrm{CHCl}_{3}\right),\left[\right.$ lit. $\left.^{4}:[\alpha]_{\mathrm{D}}^{20}+34.3\left(c=0.95, \mathrm{CHCl}_{3}\right)\right]$.

${ }^{1} \mathbf{H}$ NMR $\left(500 \mathrm{MHz}, \mathrm{CDCl}_{3}\right) \delta$ 7.35-7.32 (m, 2H), 7.27-7.24 (m, 3H), 7.00-6.96 (m, 1H), 6.94-6.89 (m, 2H), 6.66 (dd, $J=7.9,1.0 \mathrm{~Hz}, 1 \mathrm{H}), 3.84$ (s, 3H), 3.06 (br. s, 4H), 2.60 (br. s, 4H), 2.39 (tt, A of 
$\left.\mathrm{AM}_{2} \mathrm{X}_{2}, J_{A M}=11.6 \mathrm{~Hz}, J_{A X}=3.4 \mathrm{~Hz}, 1 \mathrm{H}\right), 2.28-2.11(\mathrm{~m}, 4 \mathrm{H}), 1.70-1.58(\mathrm{~m}, 4 \mathrm{H}), 1.56(\mathrm{~s}, 3 \mathrm{H}), 1.40-$ $1.28(\mathrm{~m}, 2 \mathrm{H}), 1.21-0.90(\mathrm{~m}, 4 \mathrm{H})$.

${ }^{13}$ C NMR (125 MHz, $\left.\mathrm{CDCl}_{3}\right) \delta 215.41$ (e), 152.39 (e), 141.50 (e), 141.41 (e), 128.65 (o), 127.08 (o), 127.03 (o), 122.94 (o), 121.09 (o), 118.31 (o), 111.30 (o), 55.46 (o), 55.17 (e), 54.36 (e), 53.69 (e), 50.73 (e), 46.48 (o), 34.24 (e), 31.09 (e), 30.68 (e), 25.78 (e), 25.73 (e), 25.67 (e), 20.44 (o).

IR (Neat) 2934 (m), 2855 (w), 2819 (m), 1701 (m), 1596 (w), 1501 (s), 1448 (m), 1240 (vs), 1143 (m), $1030(\mathrm{~m}), 984(\mathrm{w}), 702(\mathrm{~m}) \mathrm{cm}^{-1}$.

HRMS (EI, $\mathrm{M}^{+}$) calcd for $\mathrm{C}_{28} \mathrm{H}_{38} \mathrm{~N}_{2} \mathrm{O}_{2} 434.2933$, found 434.2939.

\section{References}

1. (a) Kurono, N.; Yamaguchi, M.; Suzuki, K.; Ohkuma, T. J. Org. Chem. 2005, 70, 6530. (b) Evans, P. A.; Oliver, S.; Chae, J. J. Am. Chem. Soc. 2012, 134, 19314.

2. Das, B.; Veeranjaneyulu, B.; Balasubramanyam , P.; Srilatha, M. Tetrahedron: Asymmetry 2010 , 21, 2762.

3. (a) Stymeist, J. L.; Bagutski, V.; French, R. M.; Aggarwal, V. K. Nature 2008, 456, 778. (b) Bagutski, V.; French, R. M.; Aggarwal, V. K. Angew. Chem. Int. Ed. 2010, 49, 5142. (c) Evans, P. A.; Oliver, S. Org. Lett. 2013, 15, 5626.

4. Denmark, S. E.; Fu, J. Org. Lett. 2002, 4, 1951. 


\section{Proton and Carbon NMR Spectra}

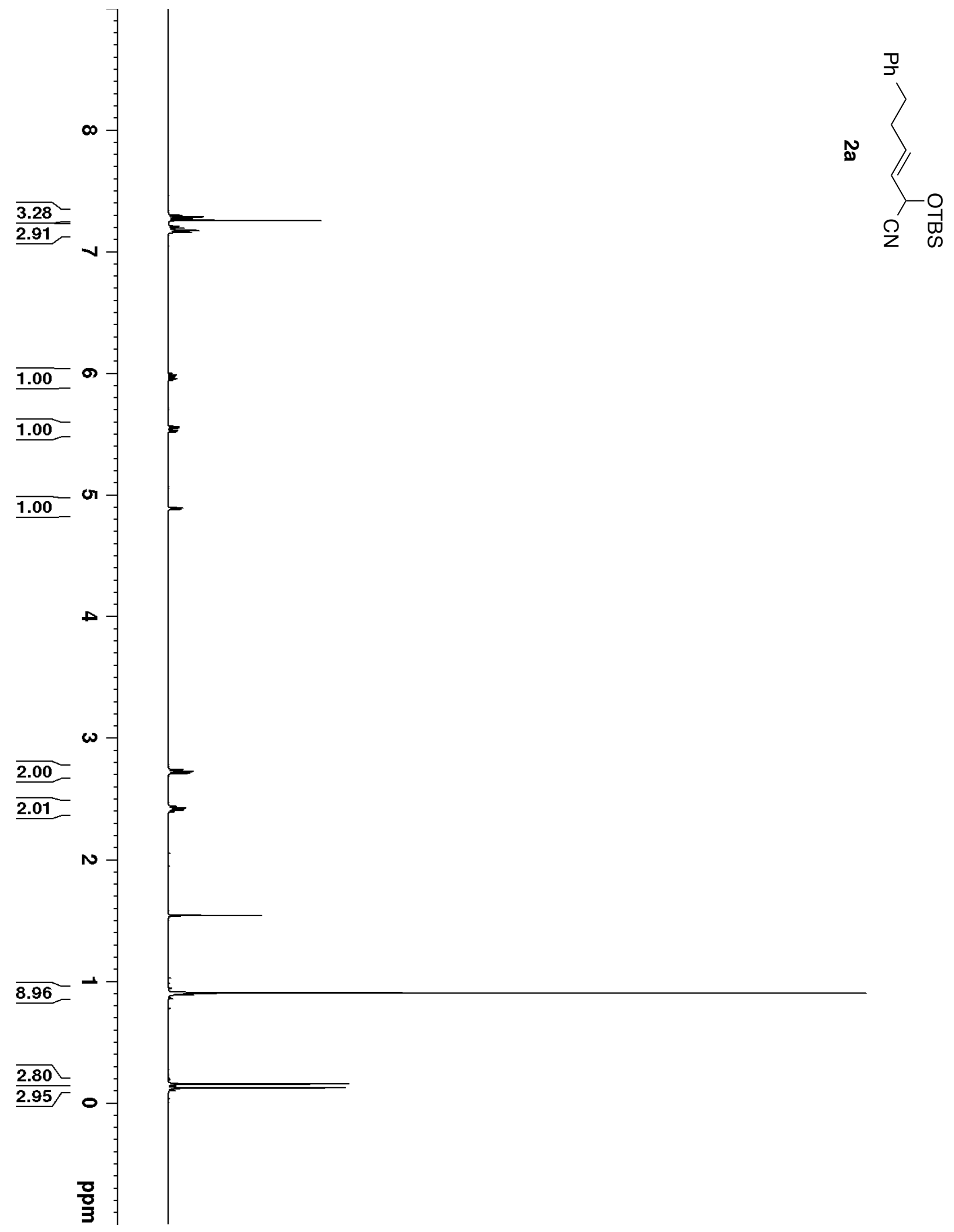




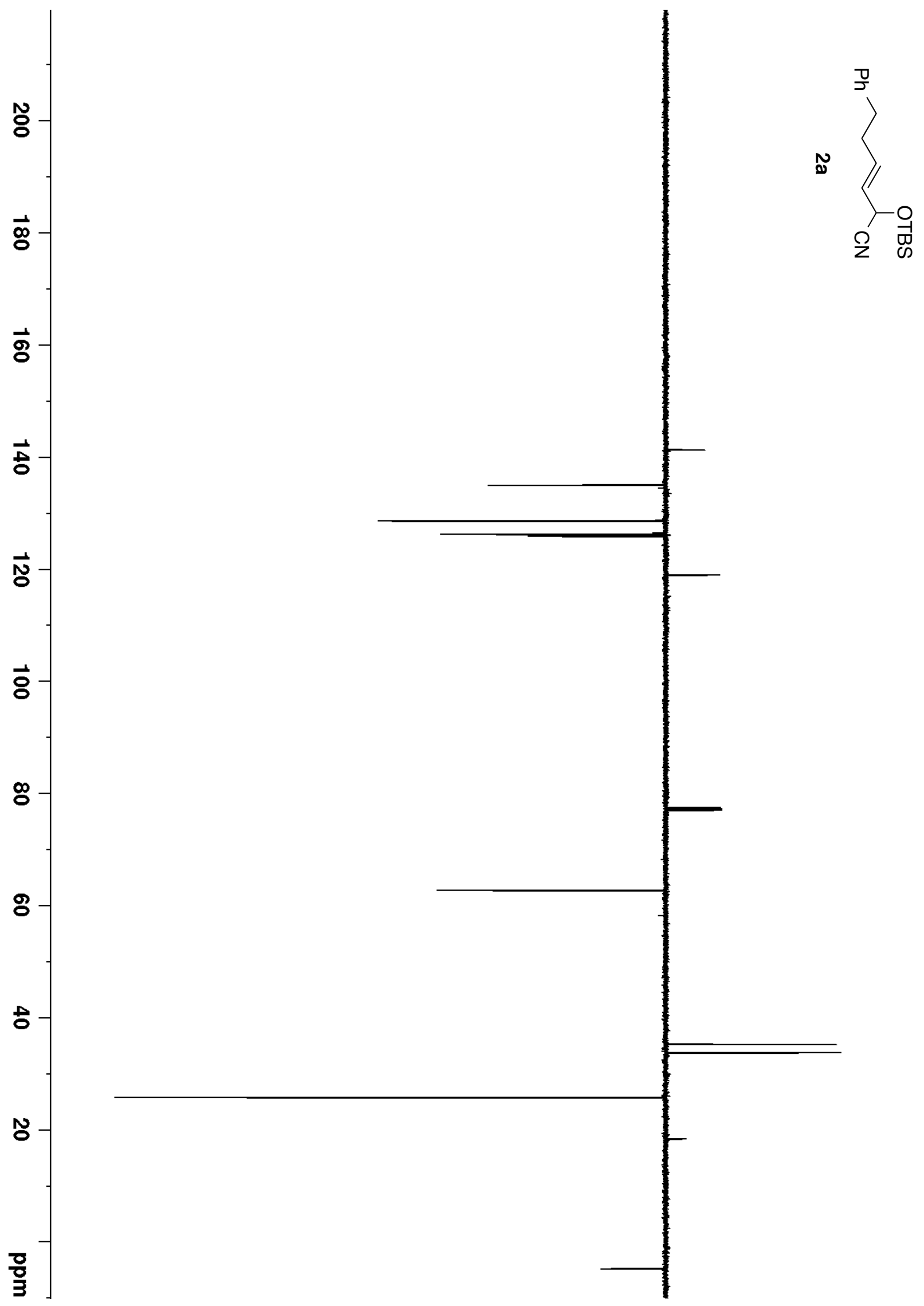




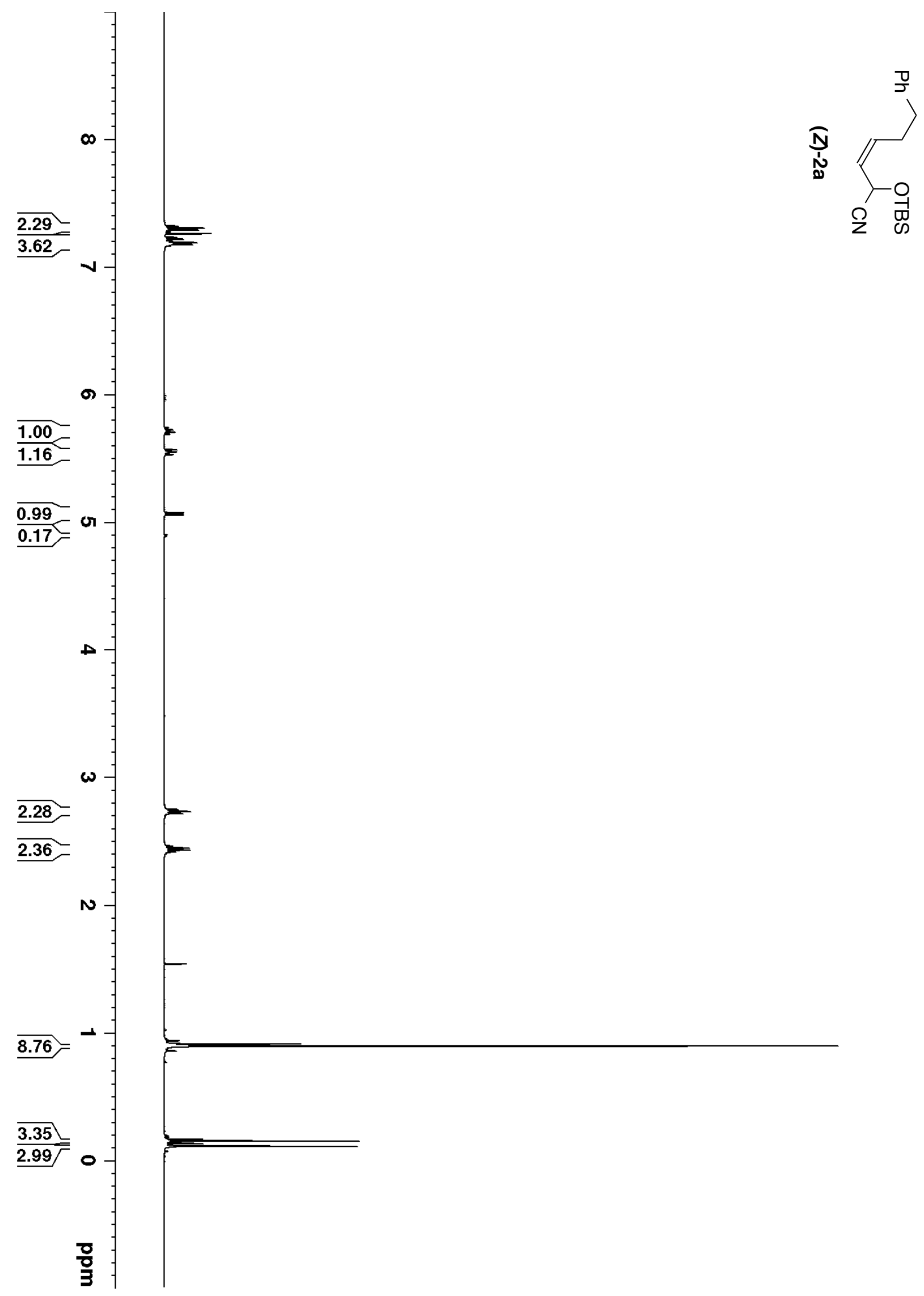




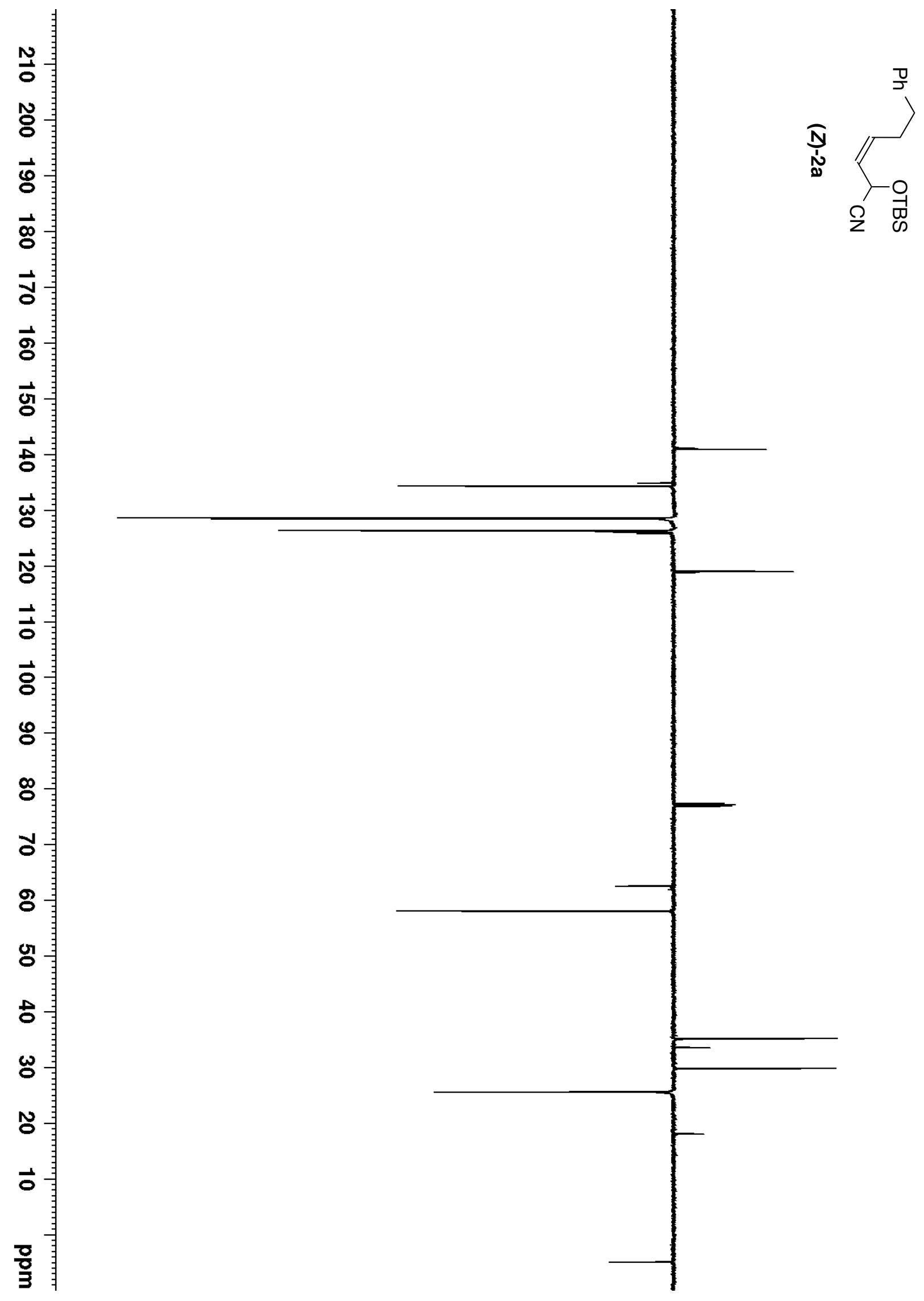




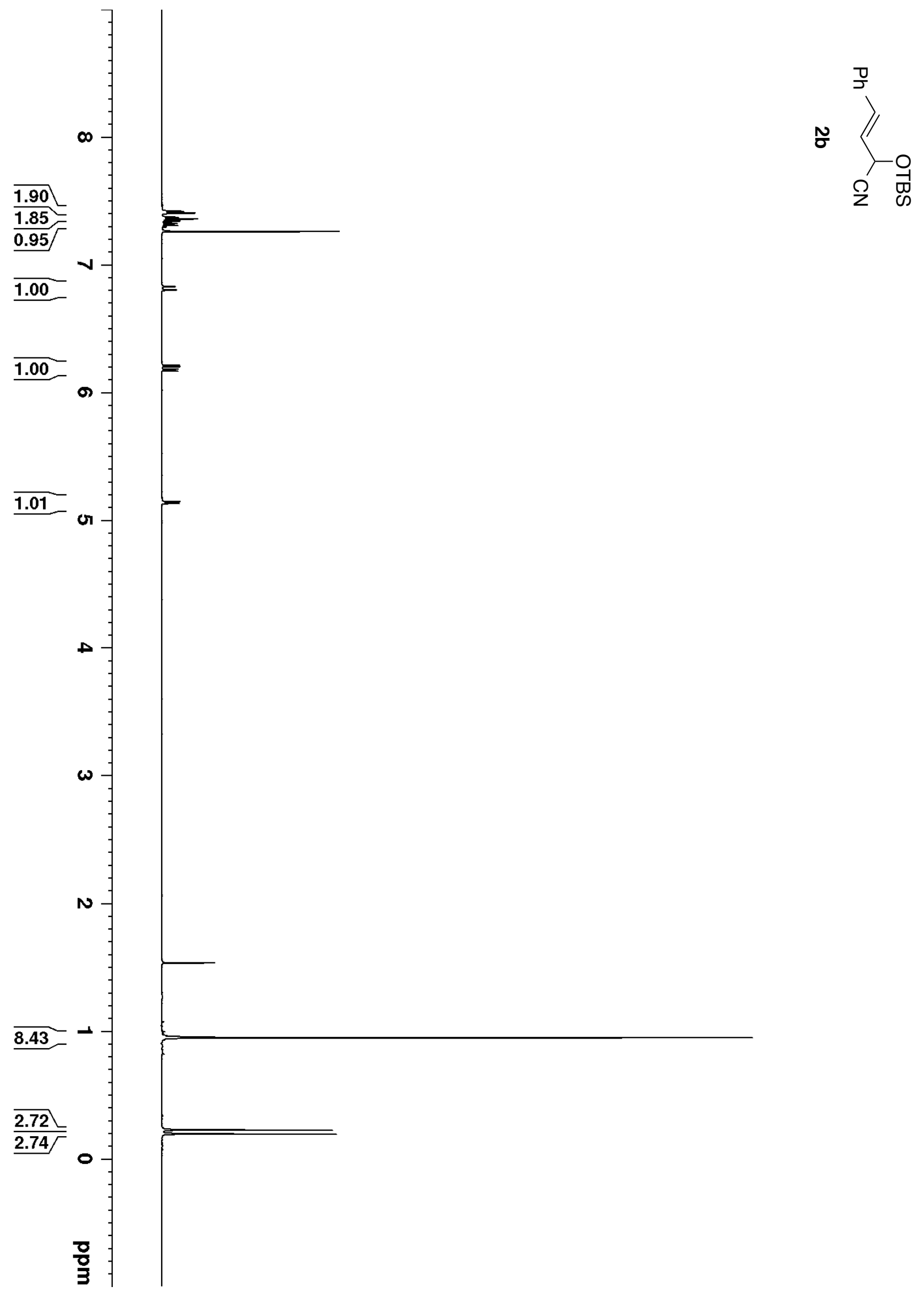




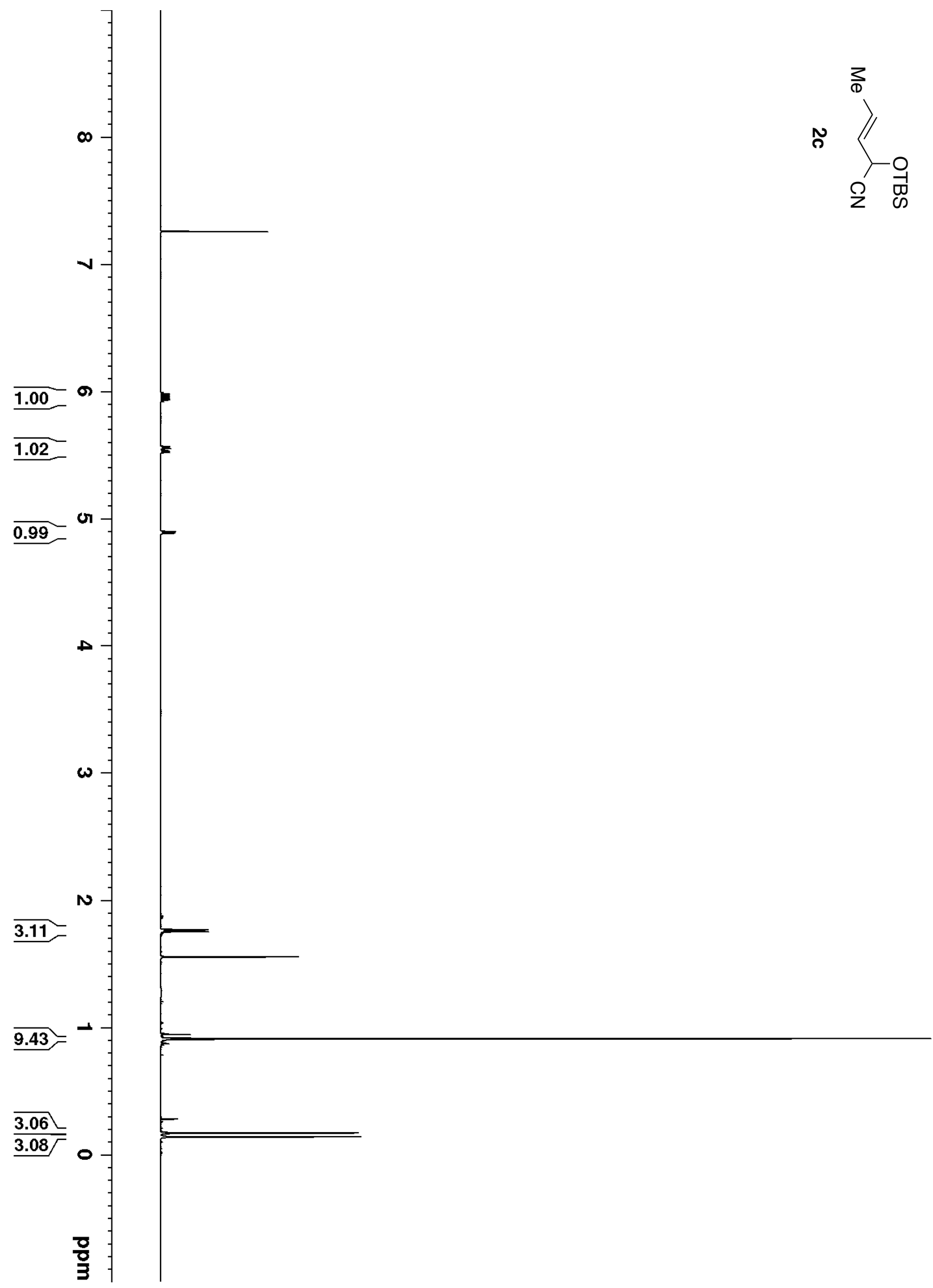




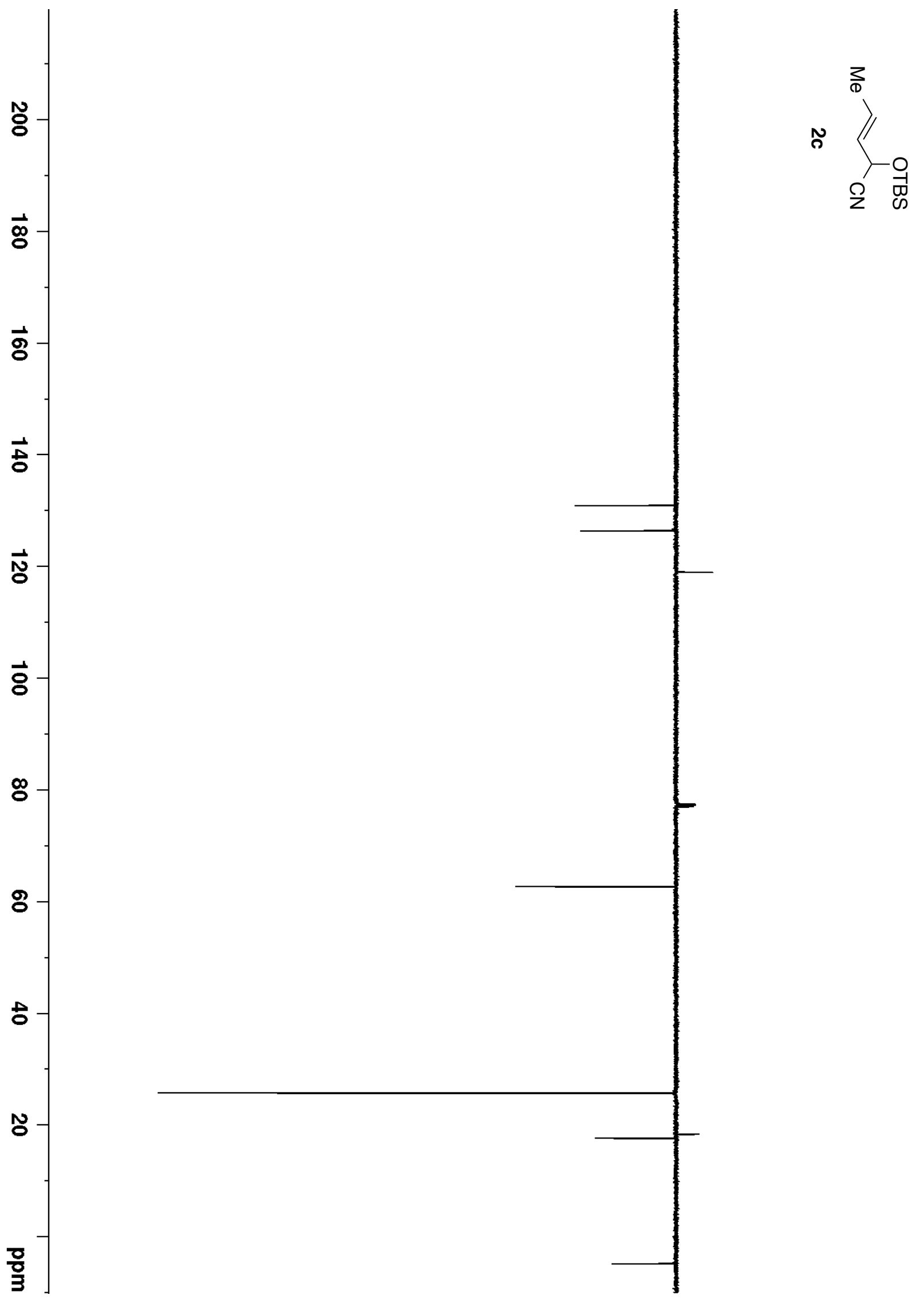



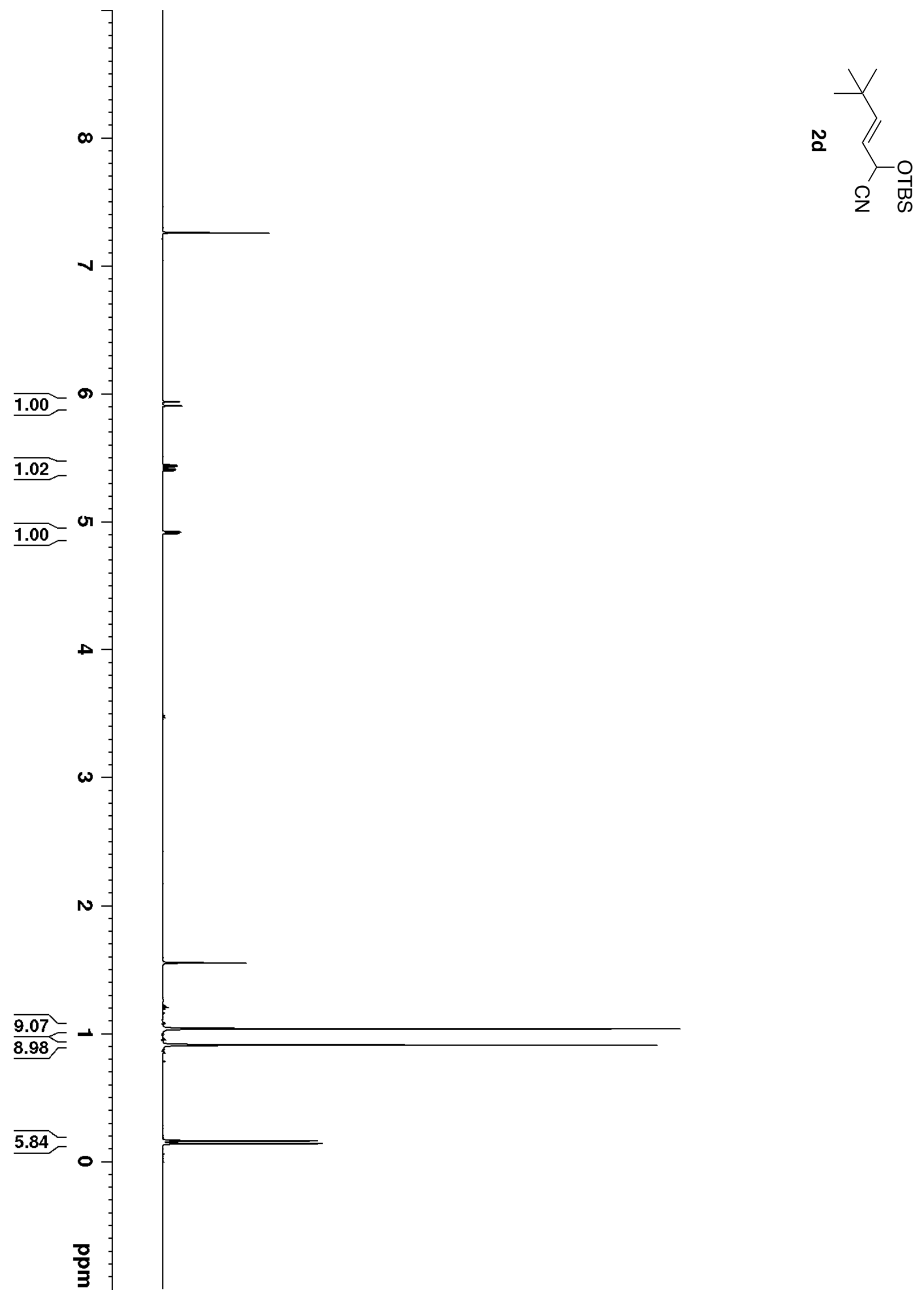


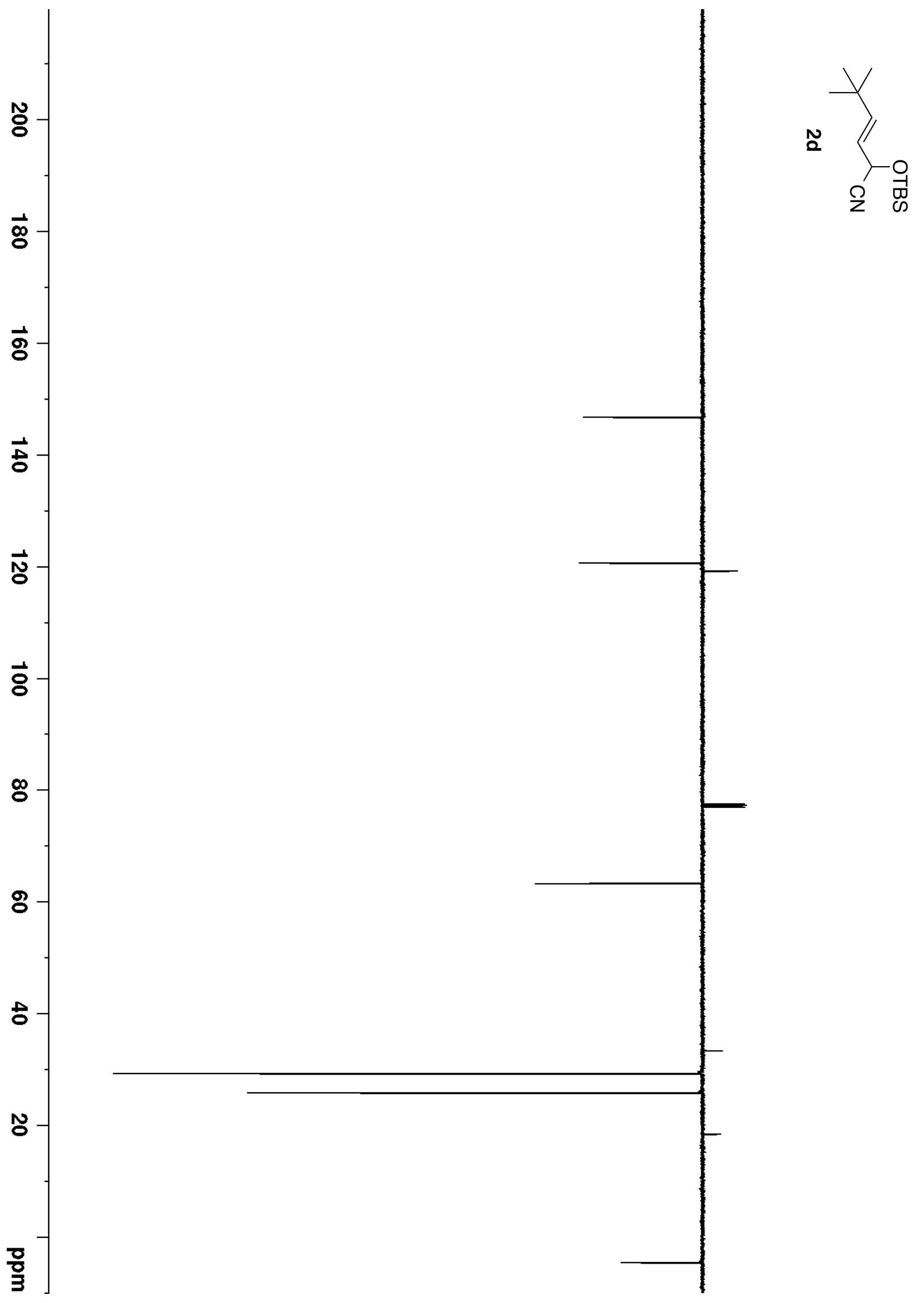



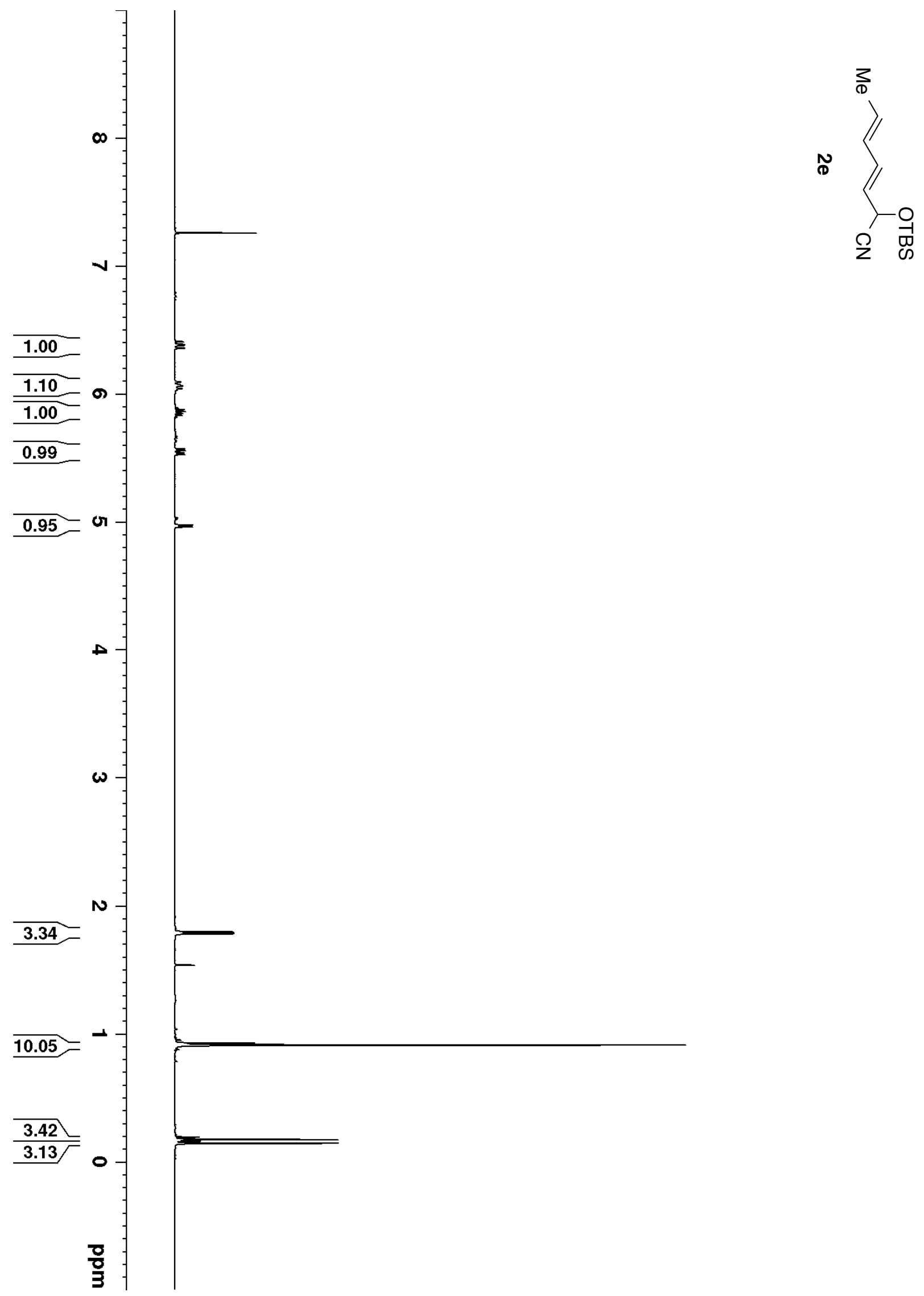


$$
f
$$



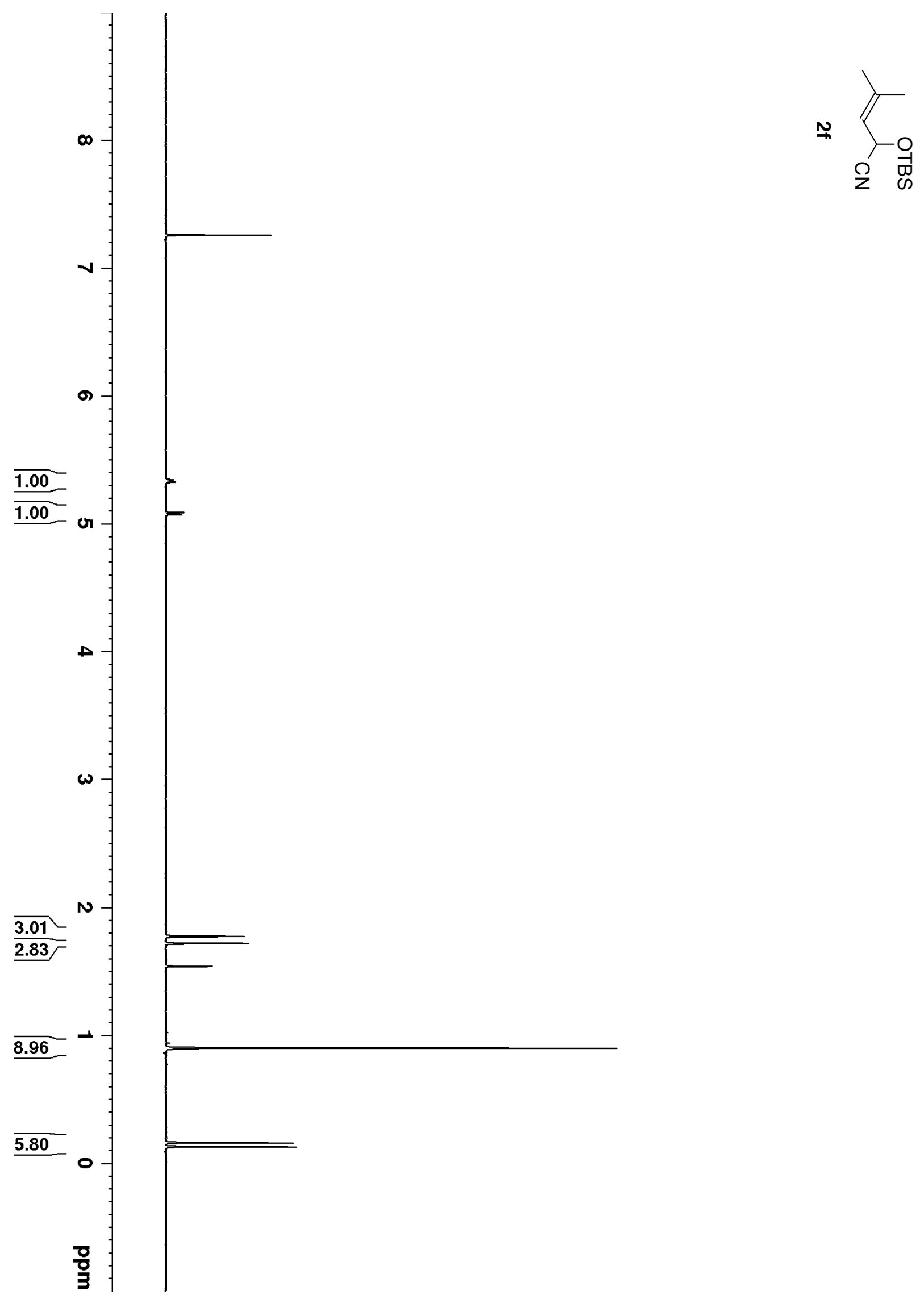


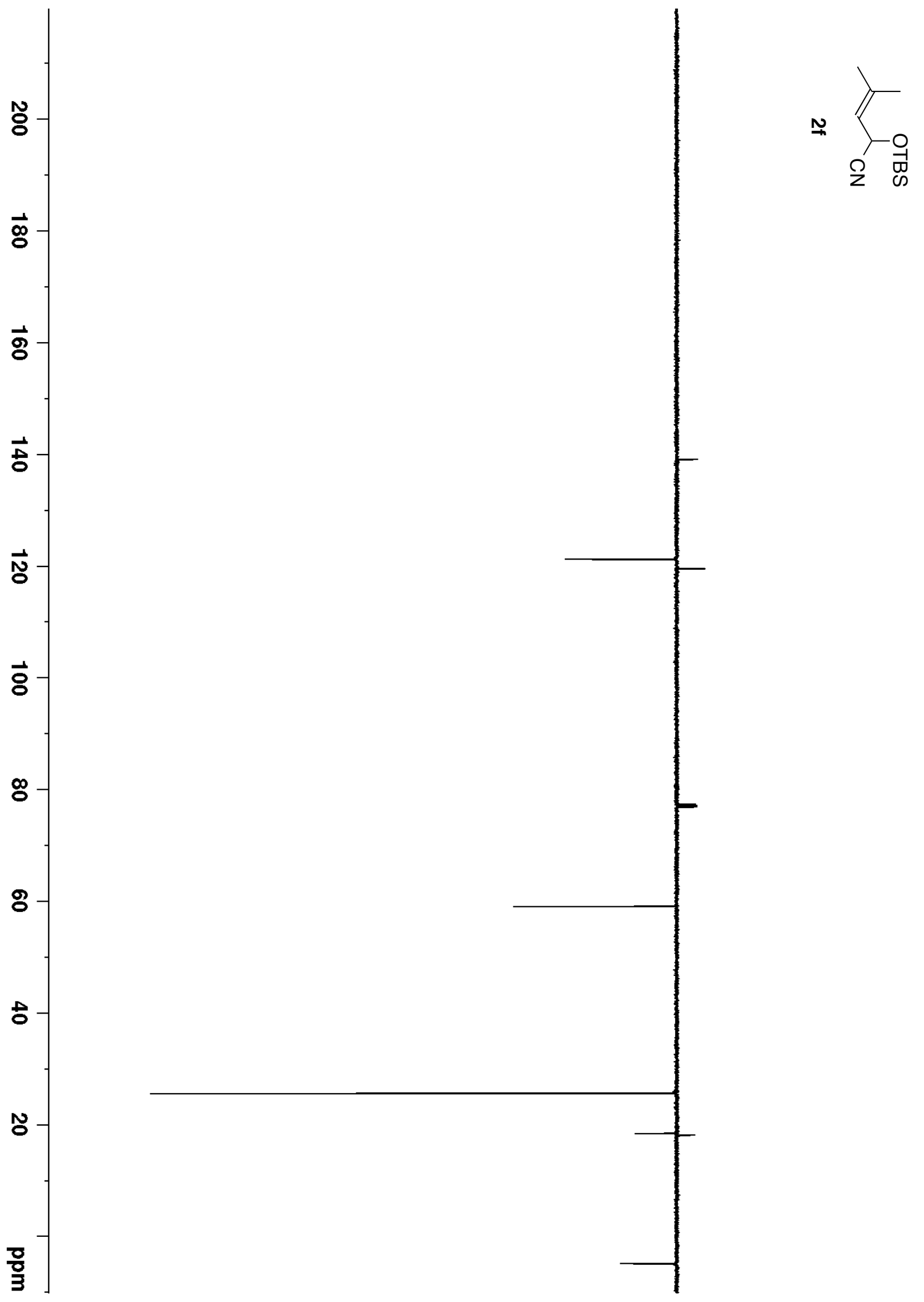




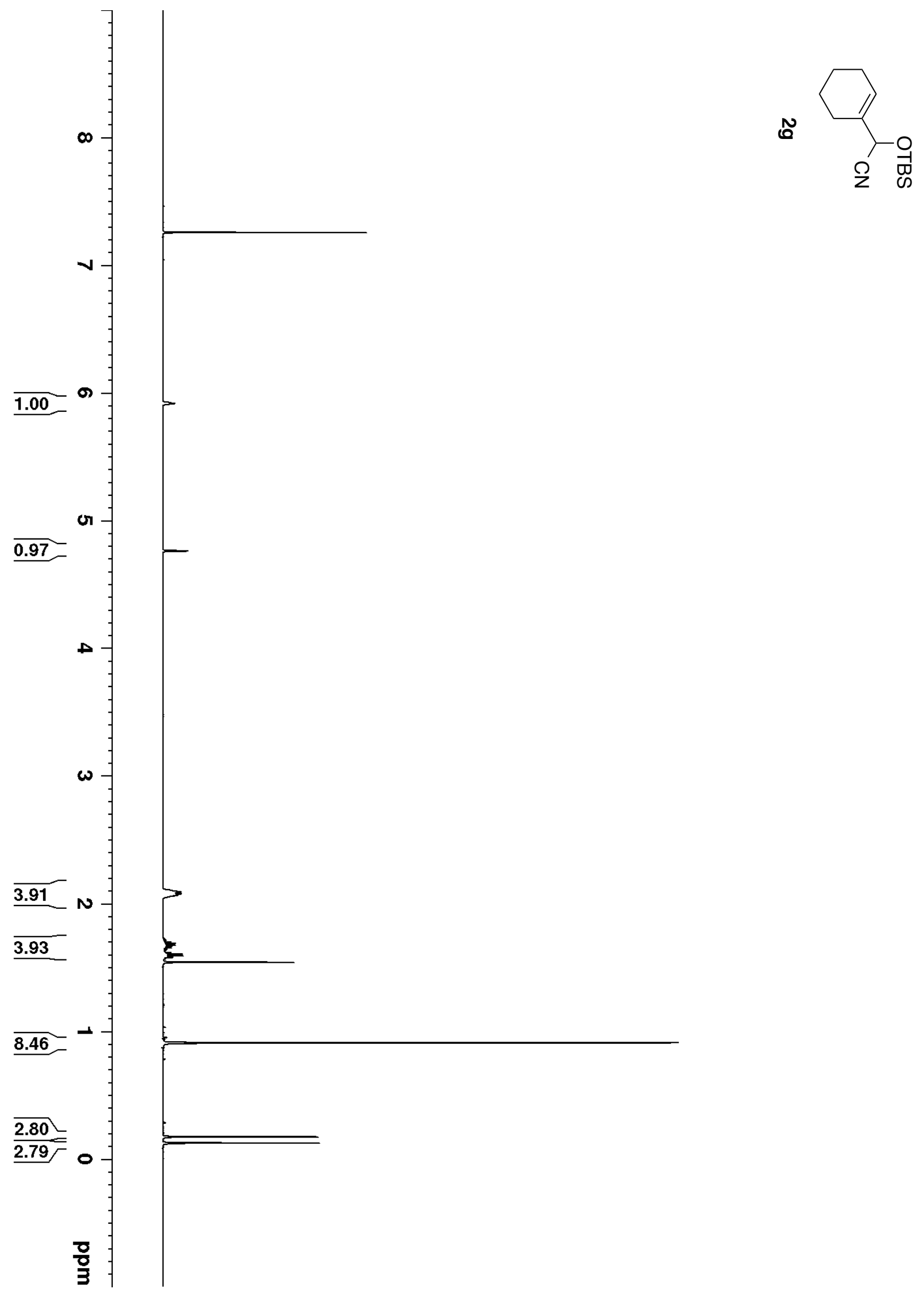




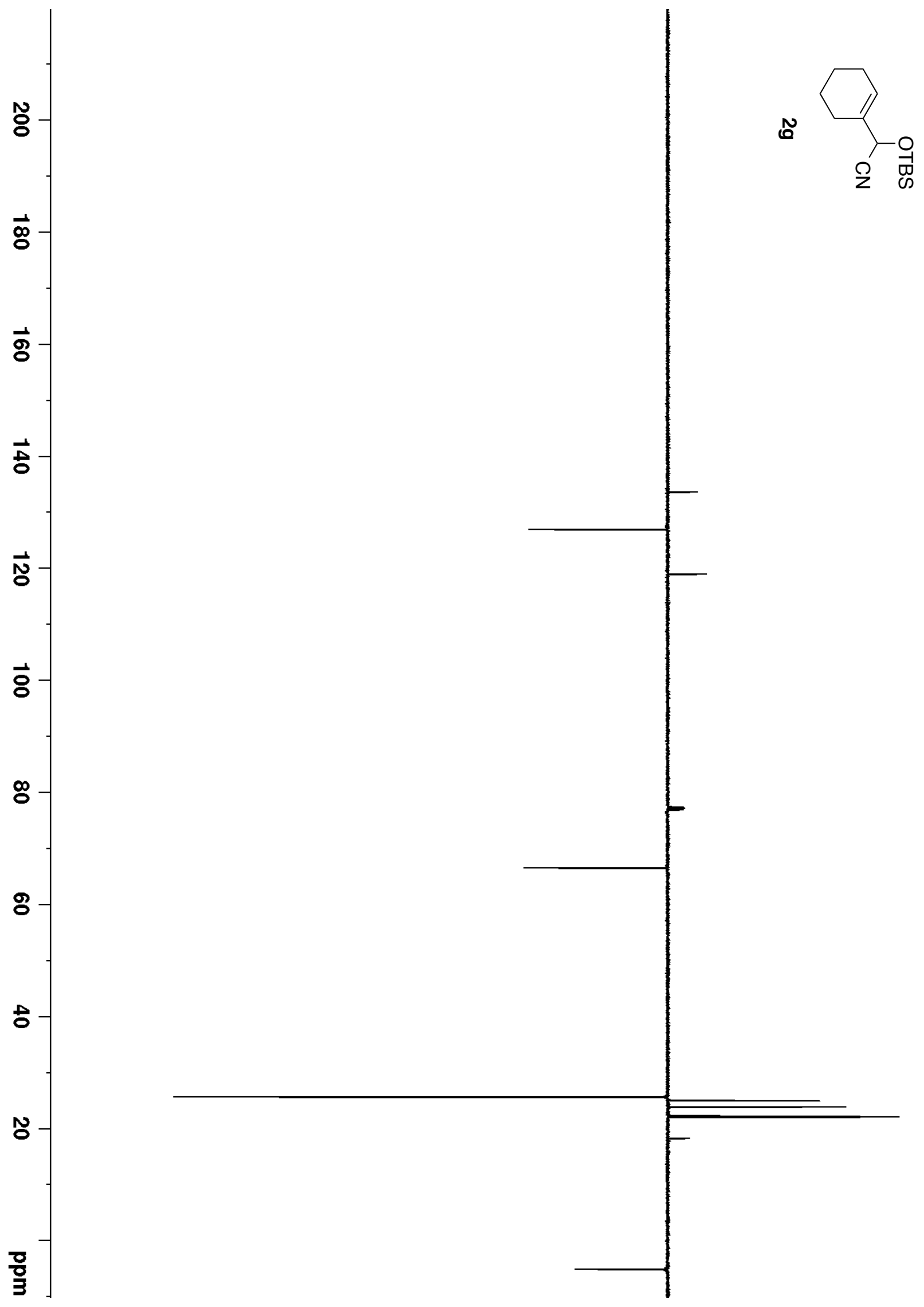



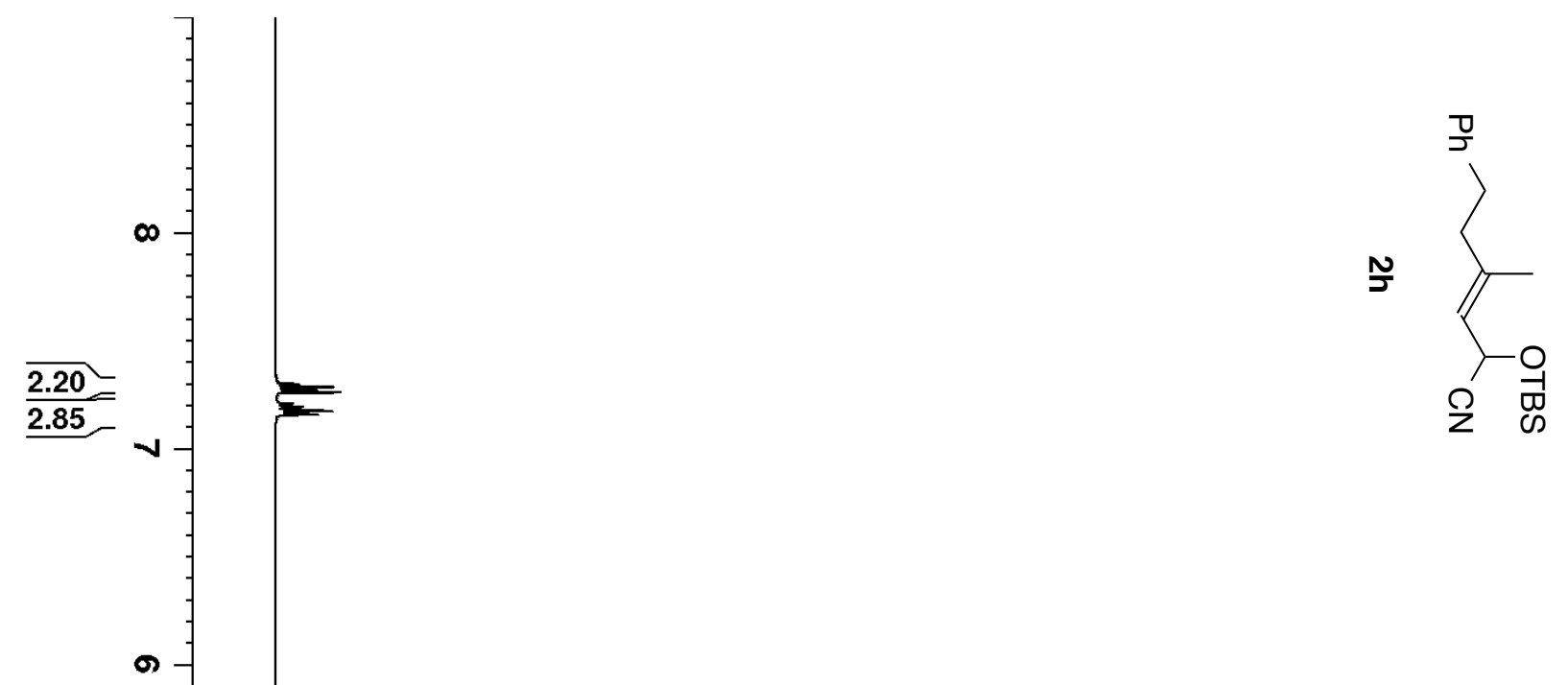


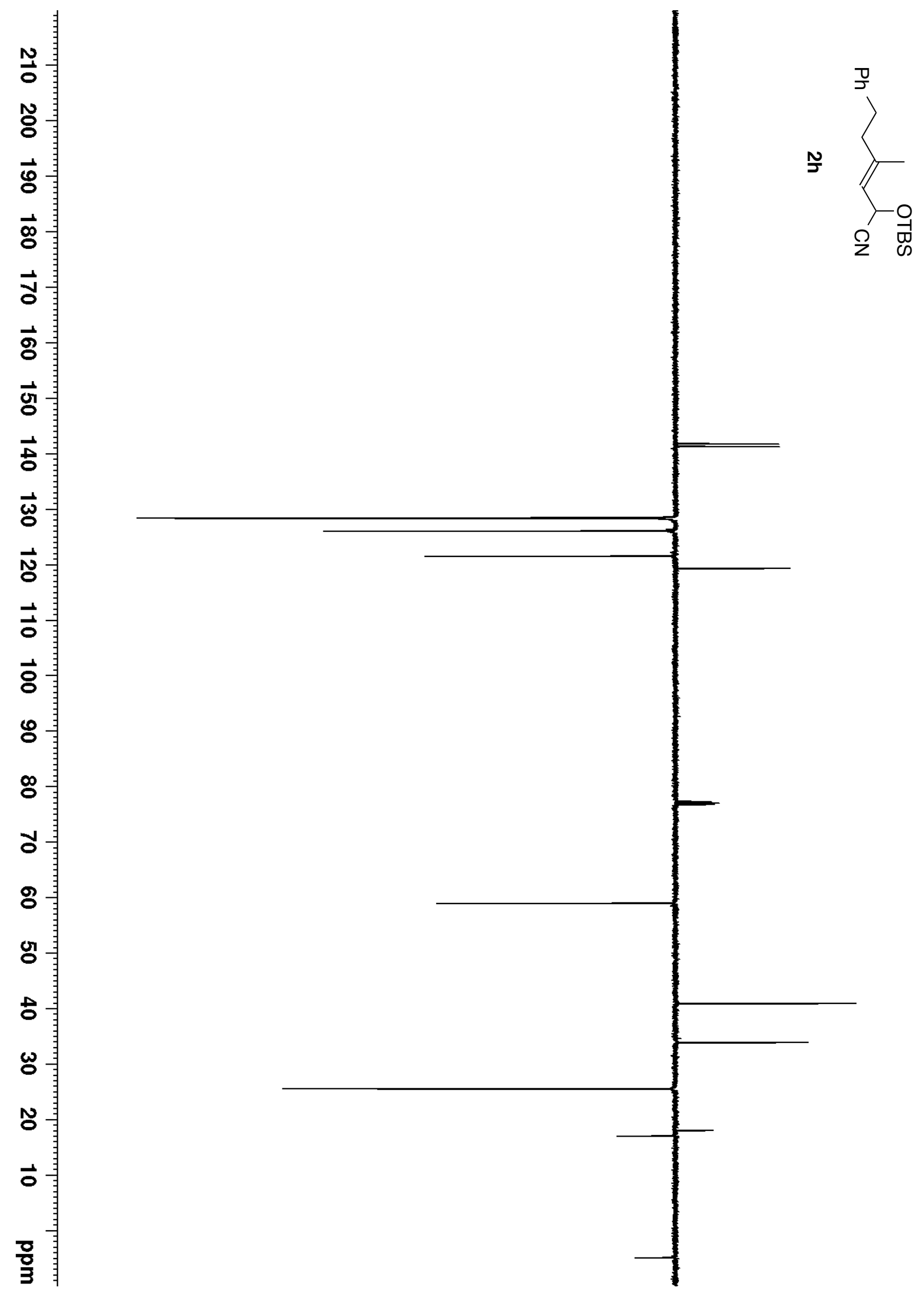




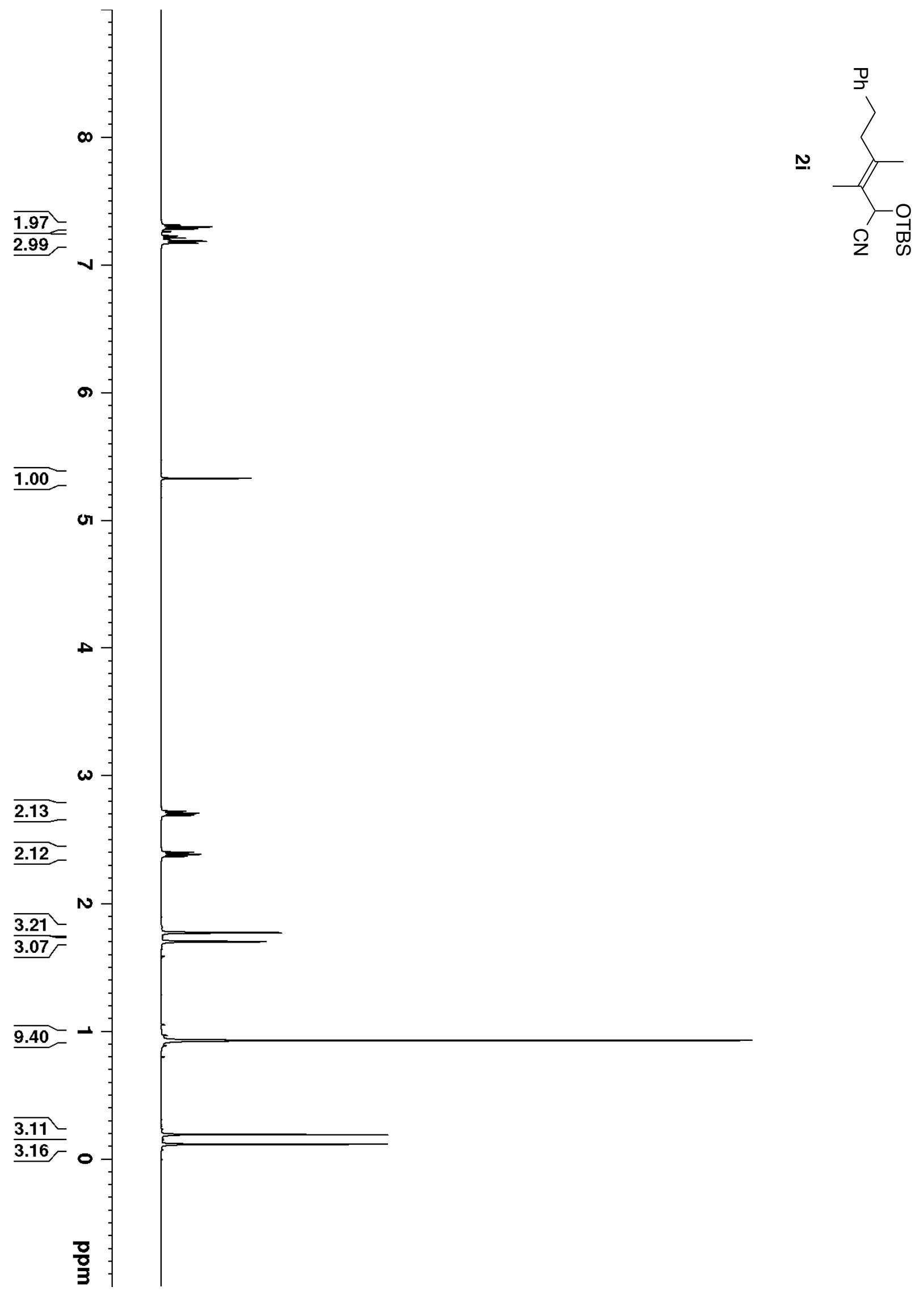




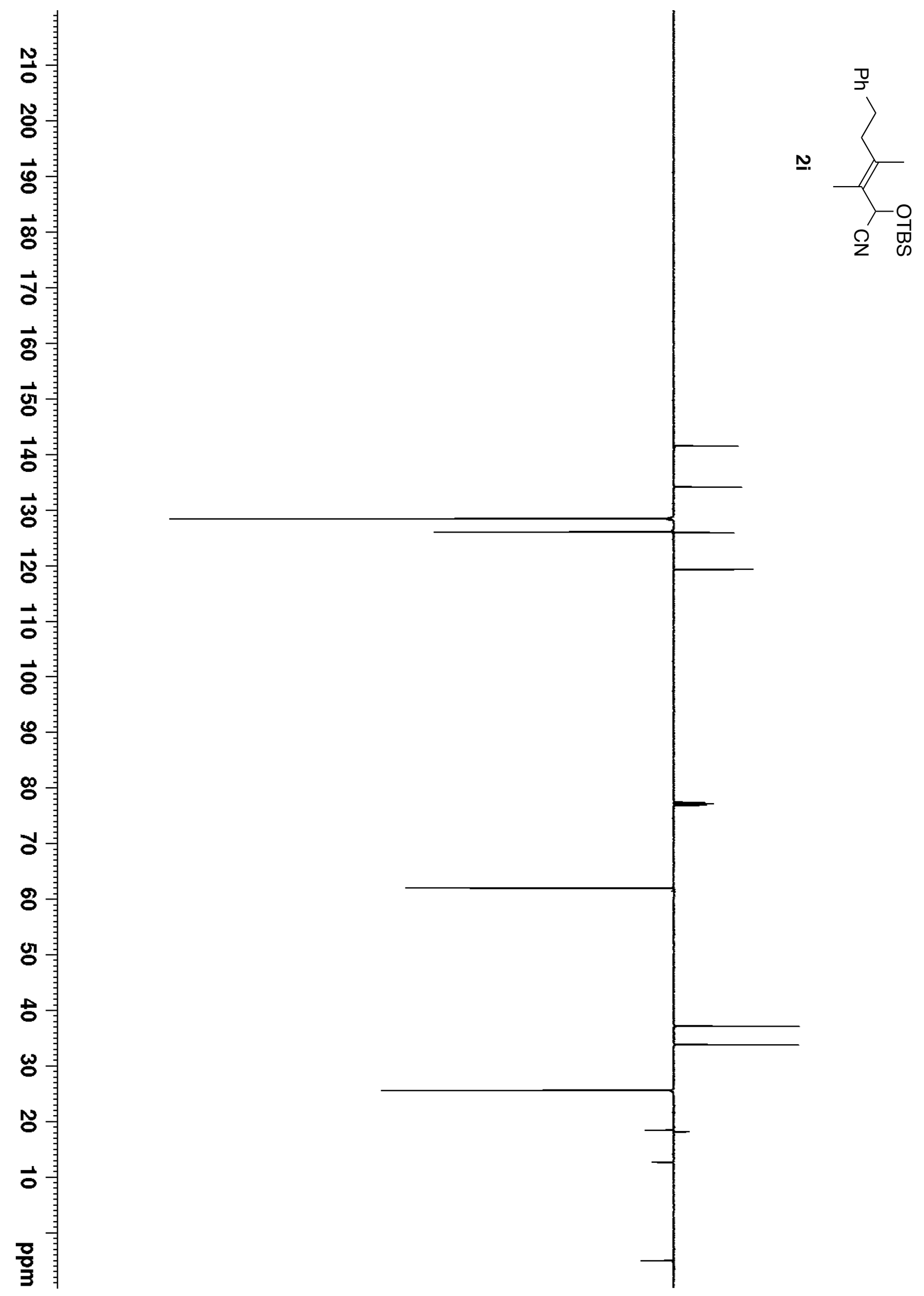




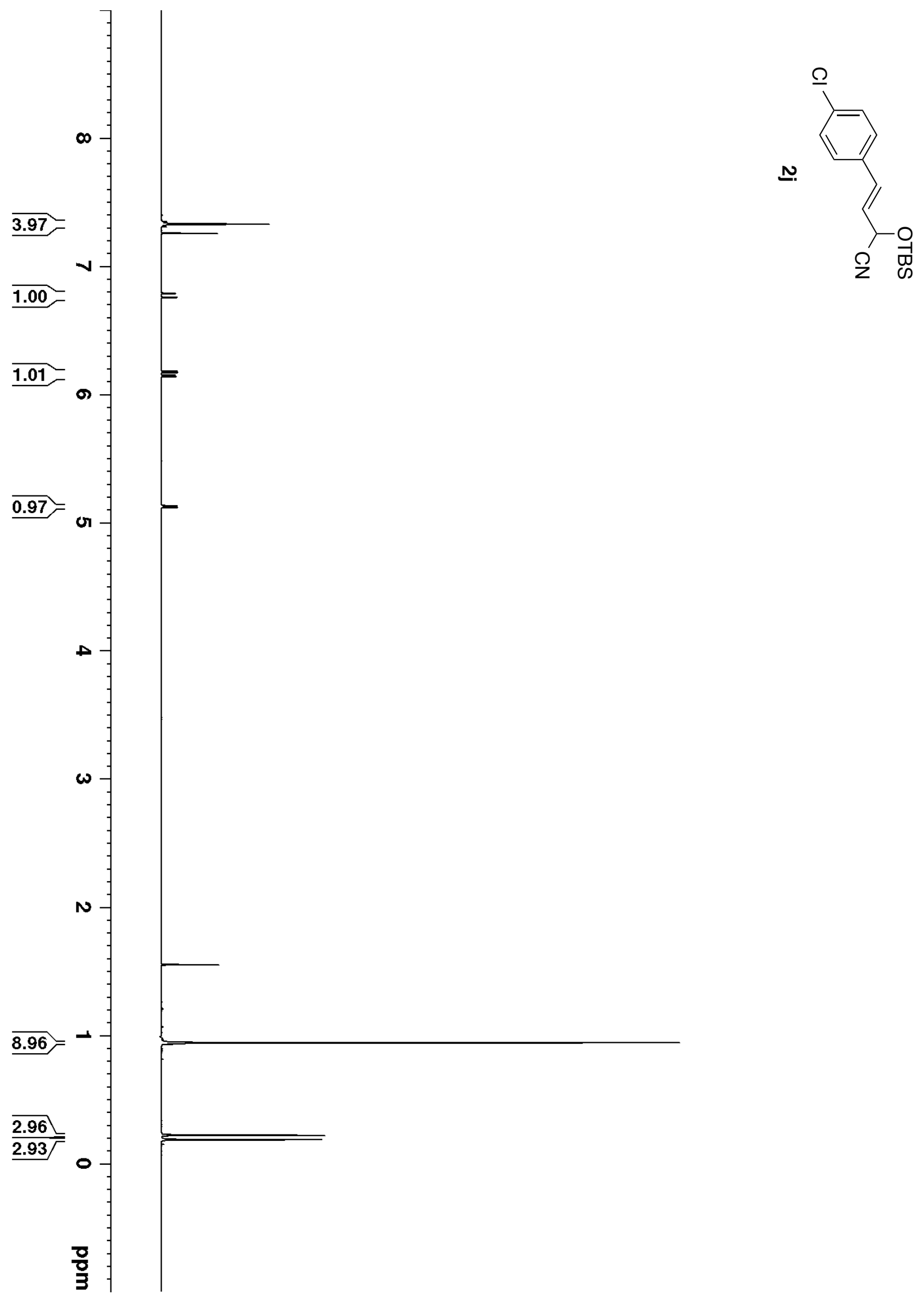




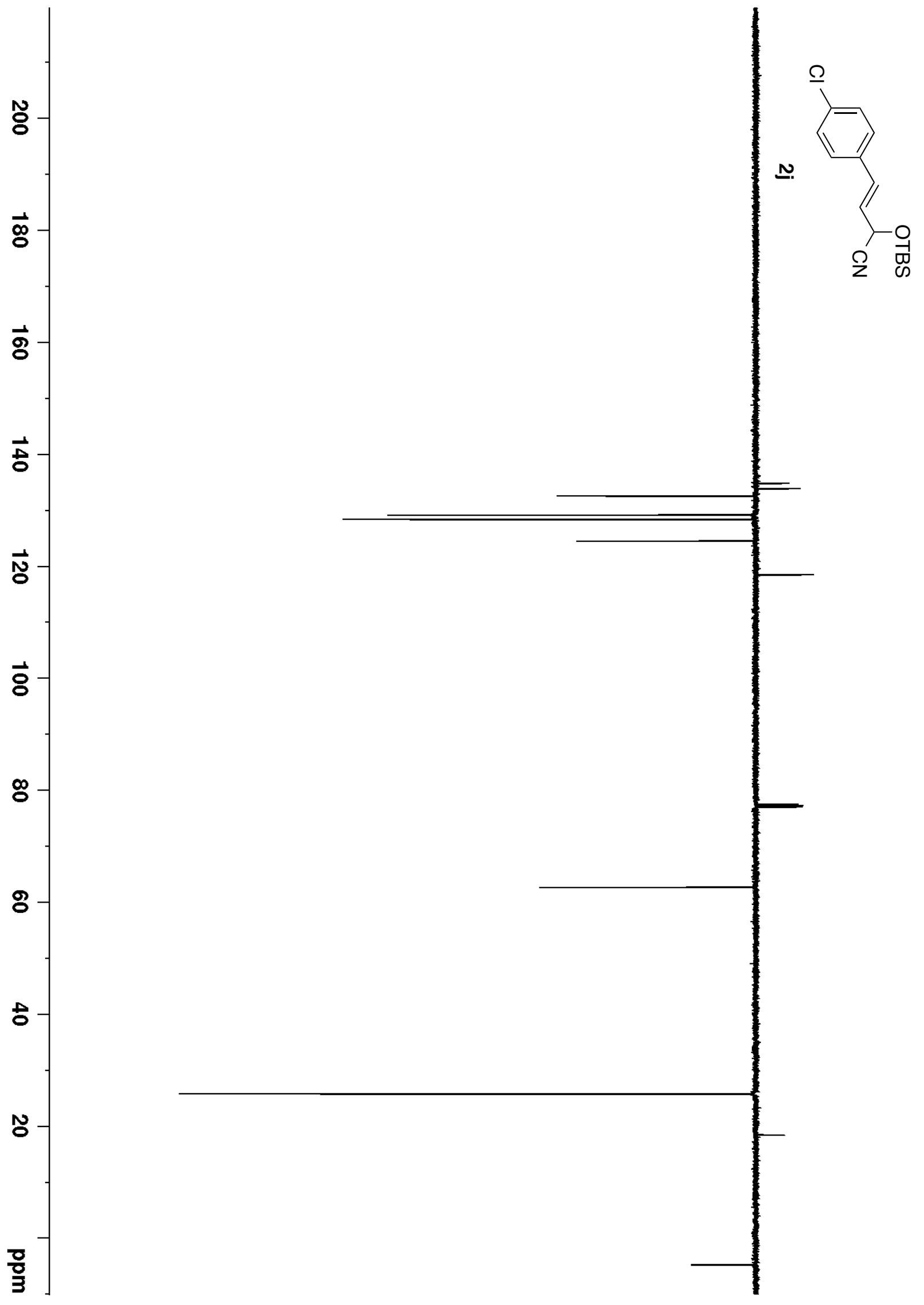



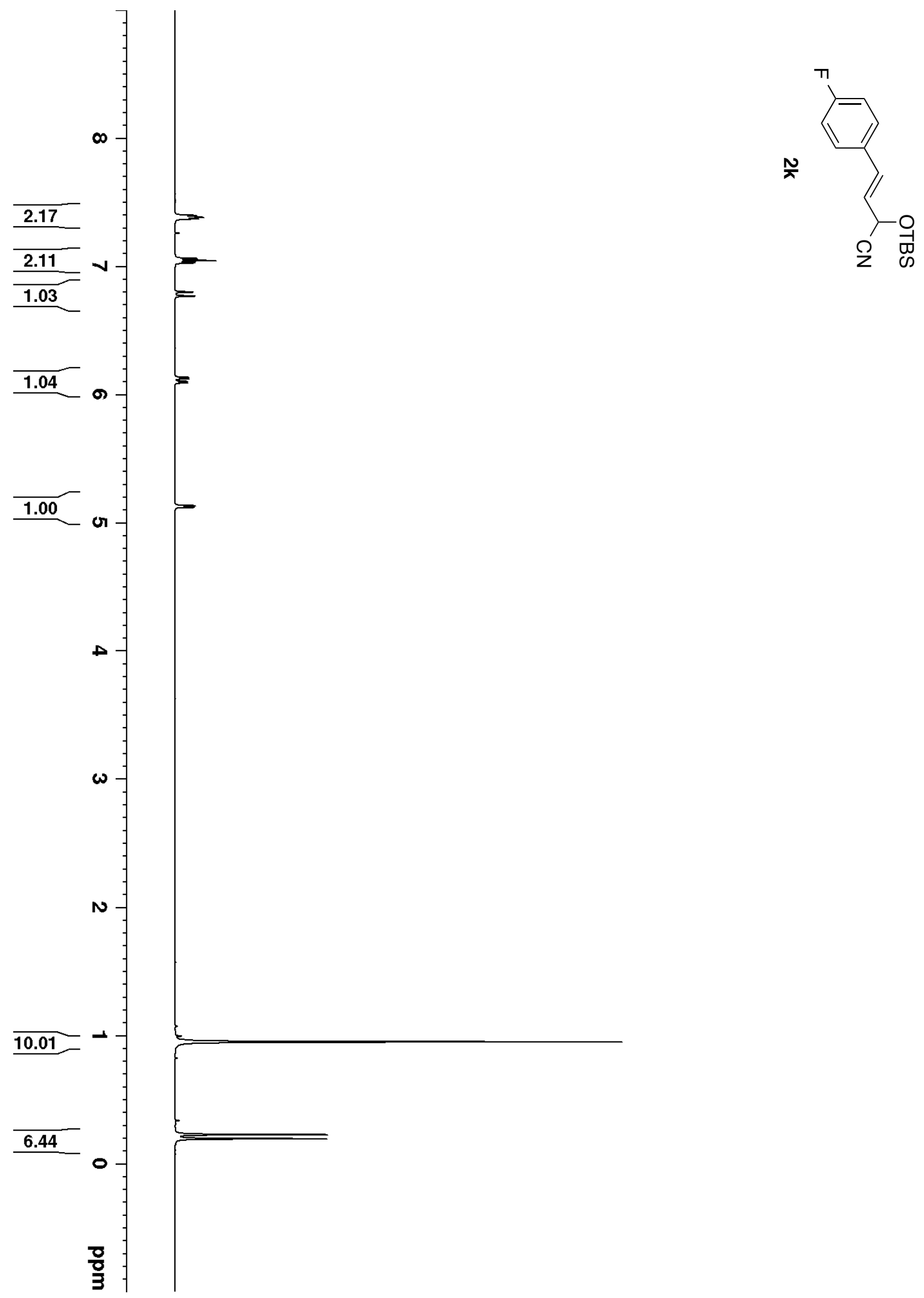


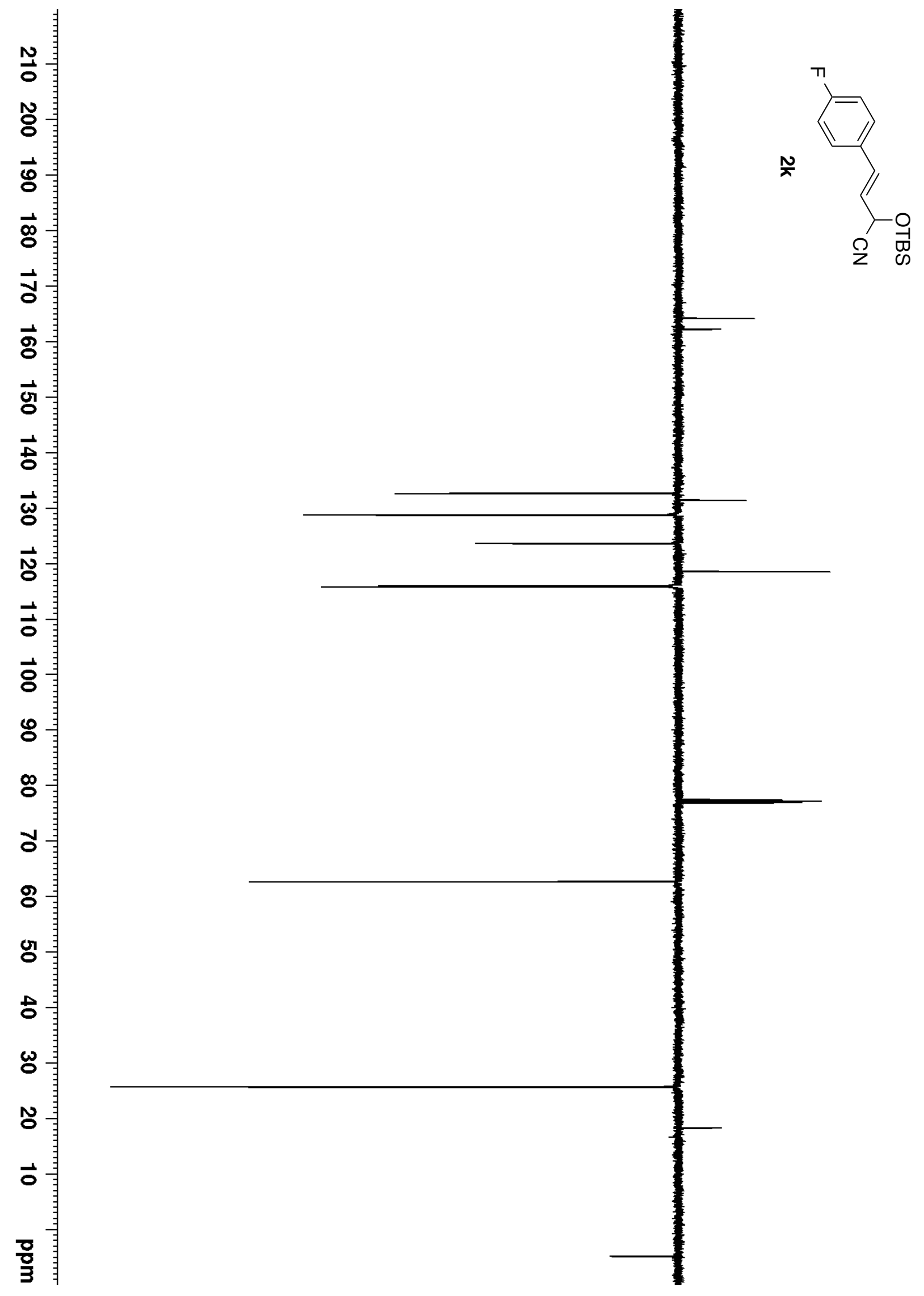



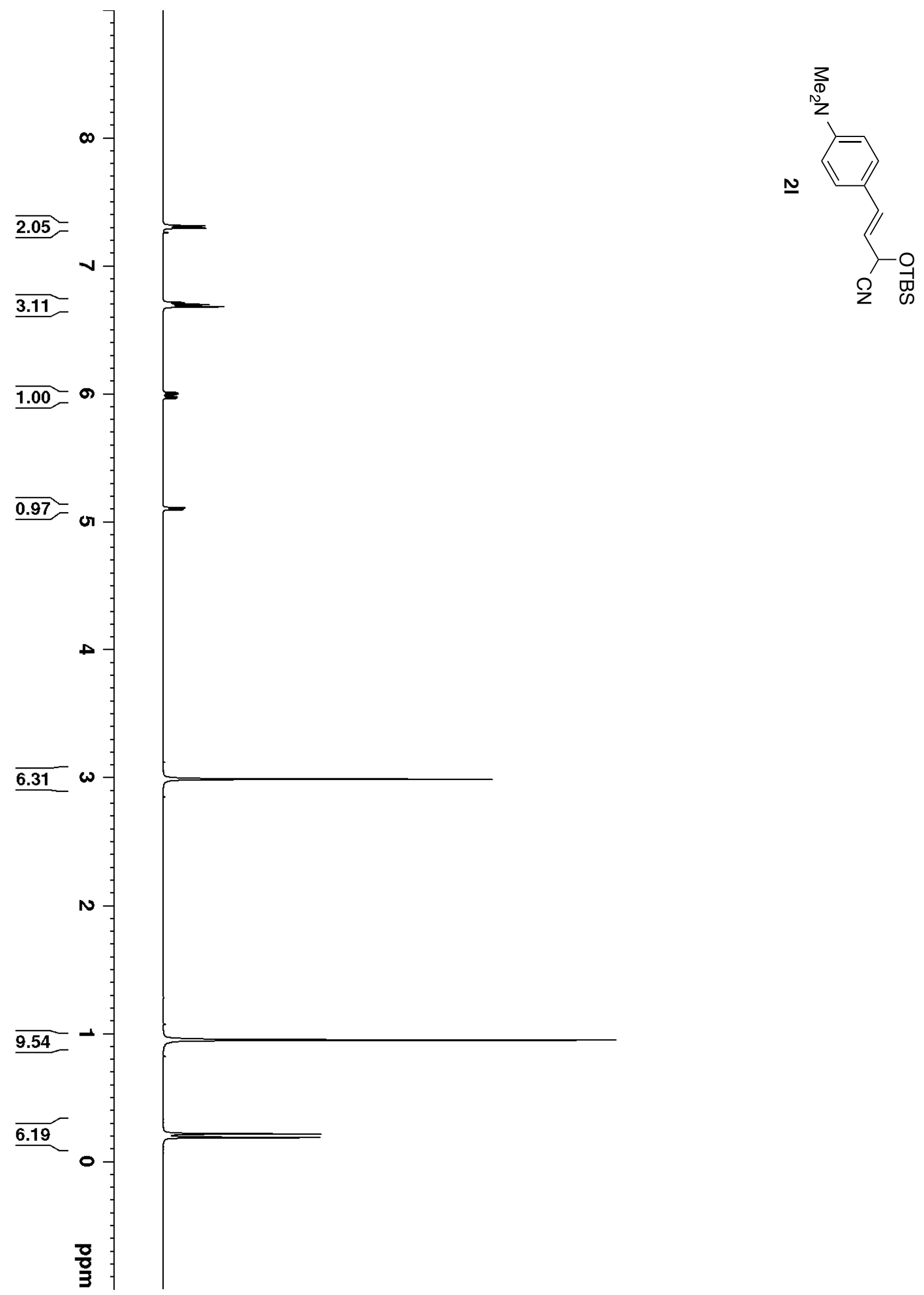


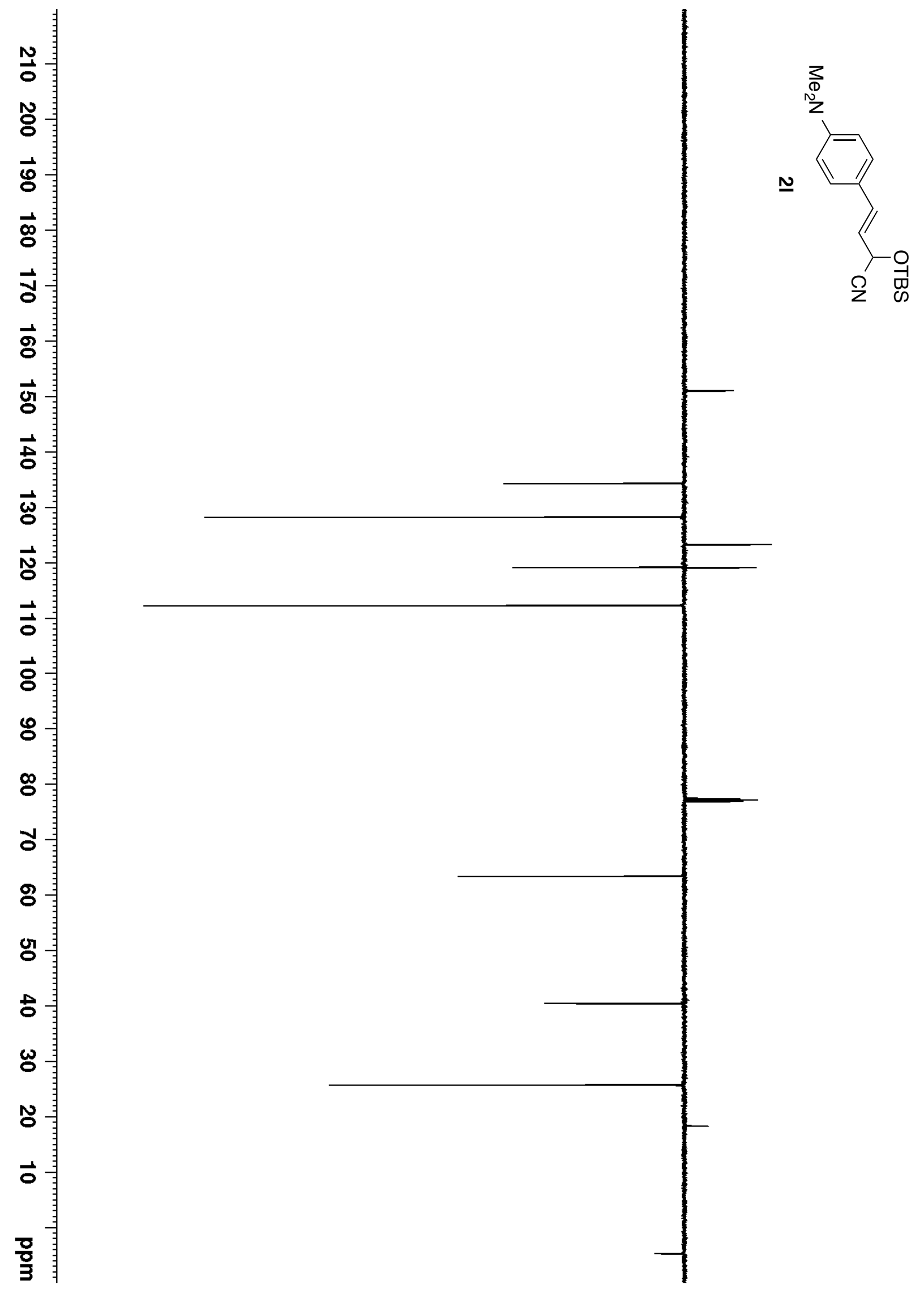



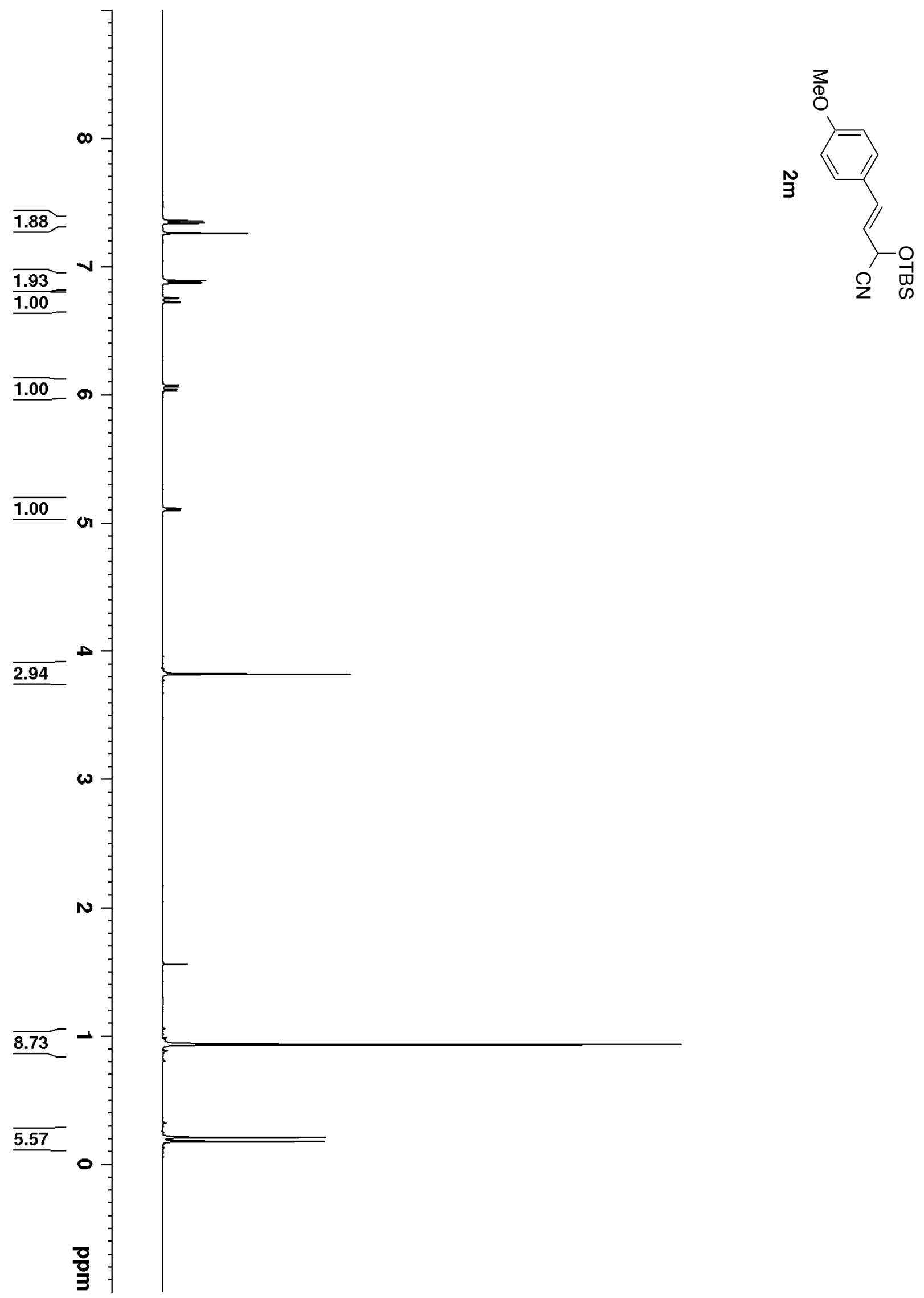


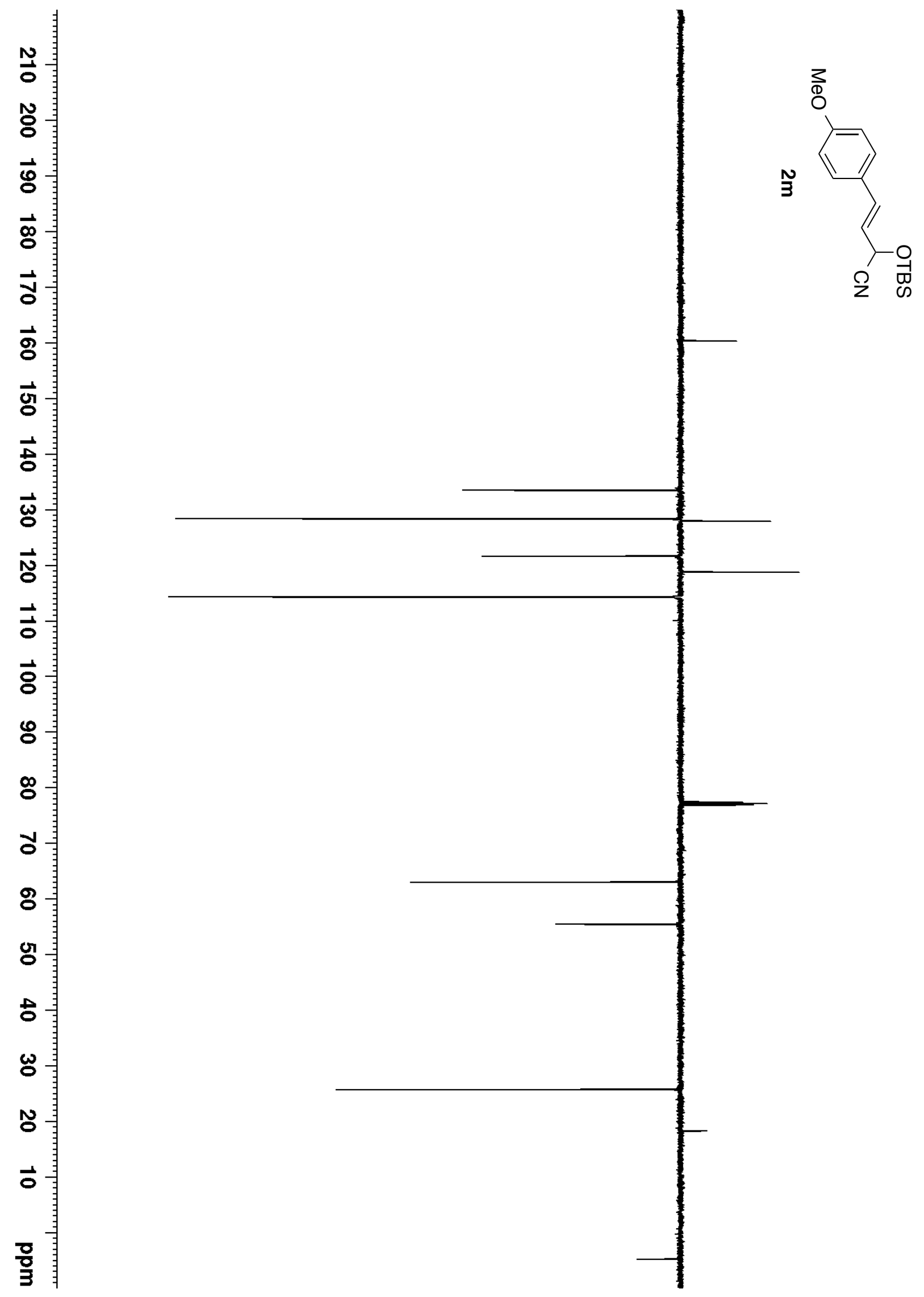



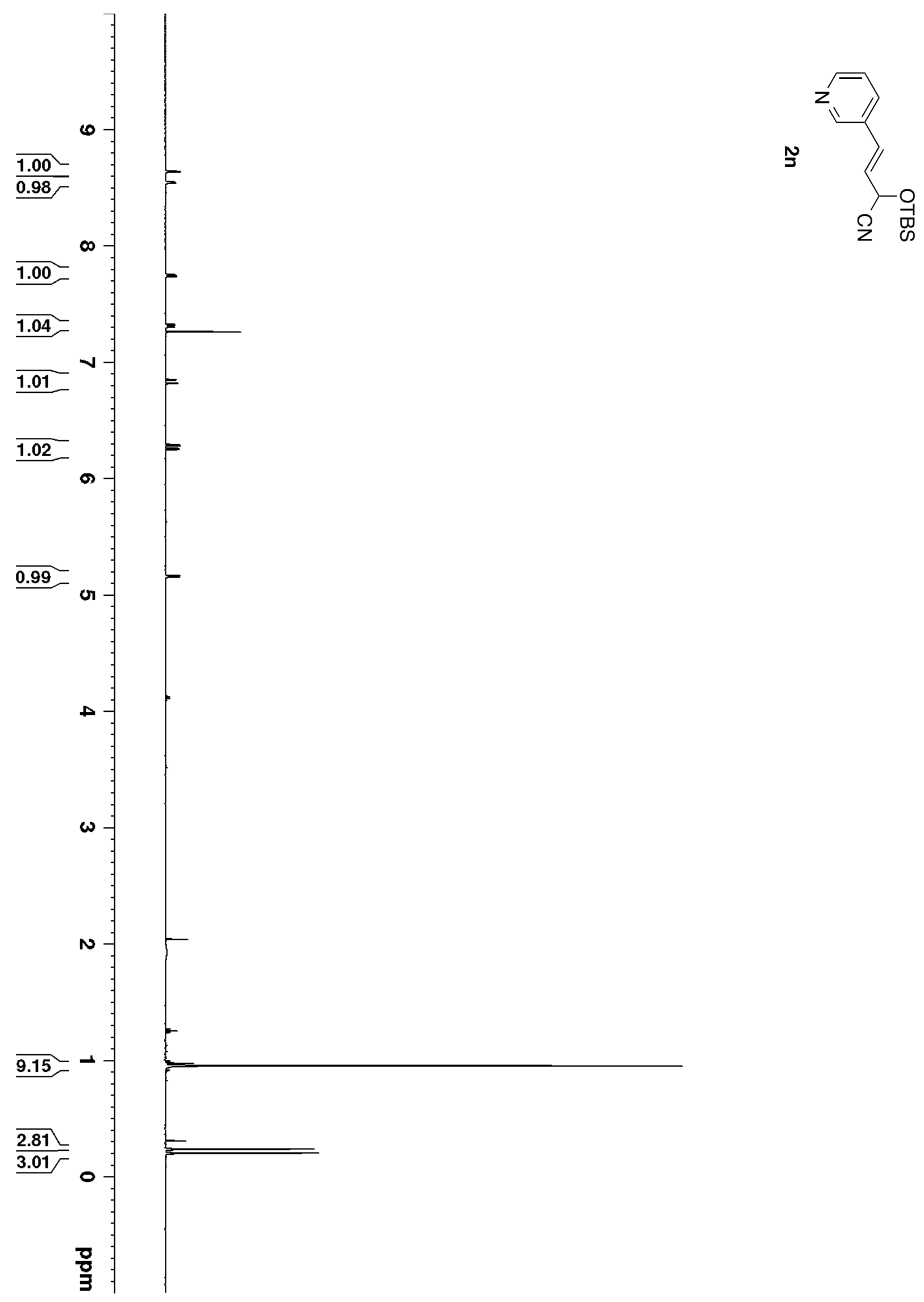


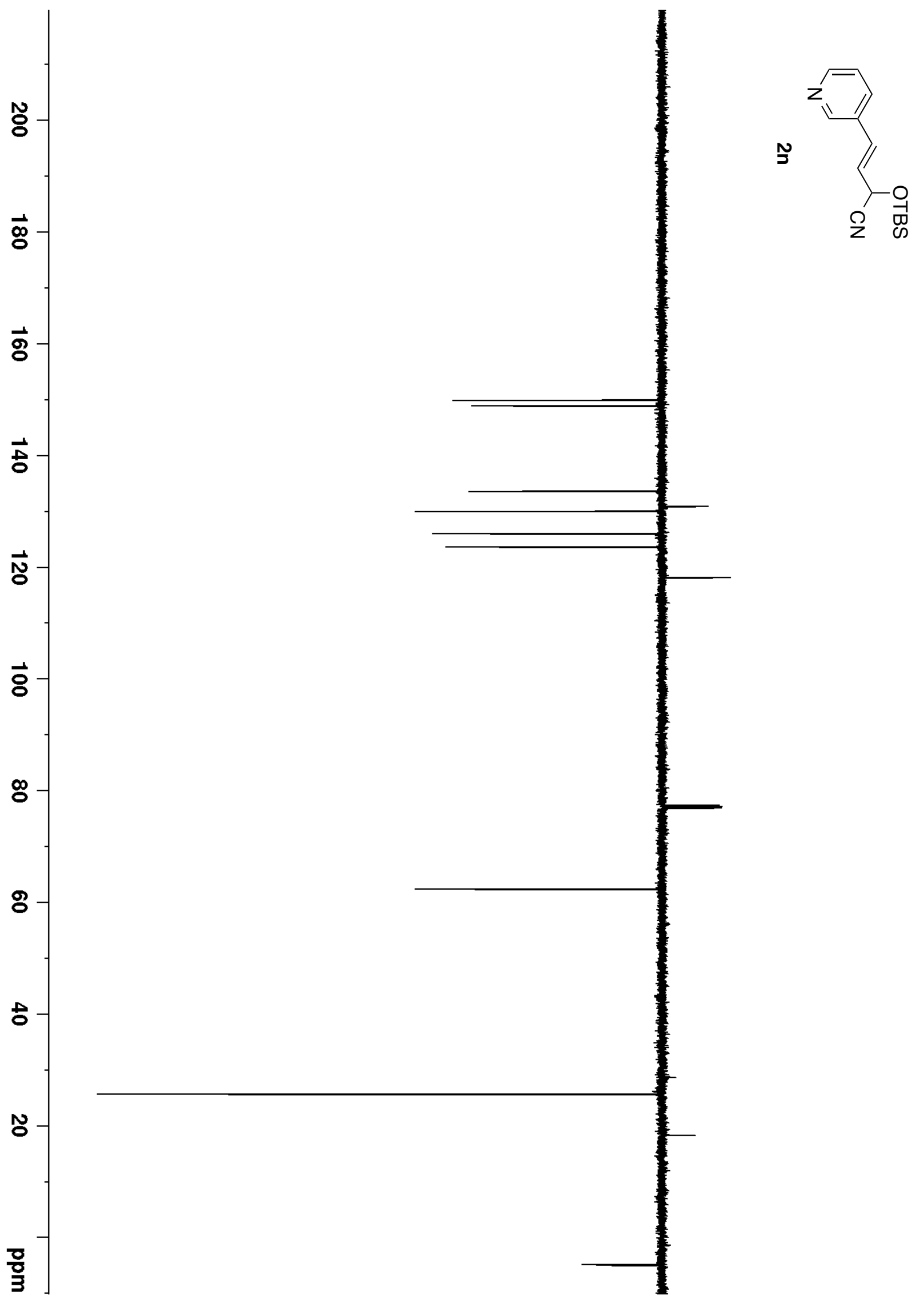



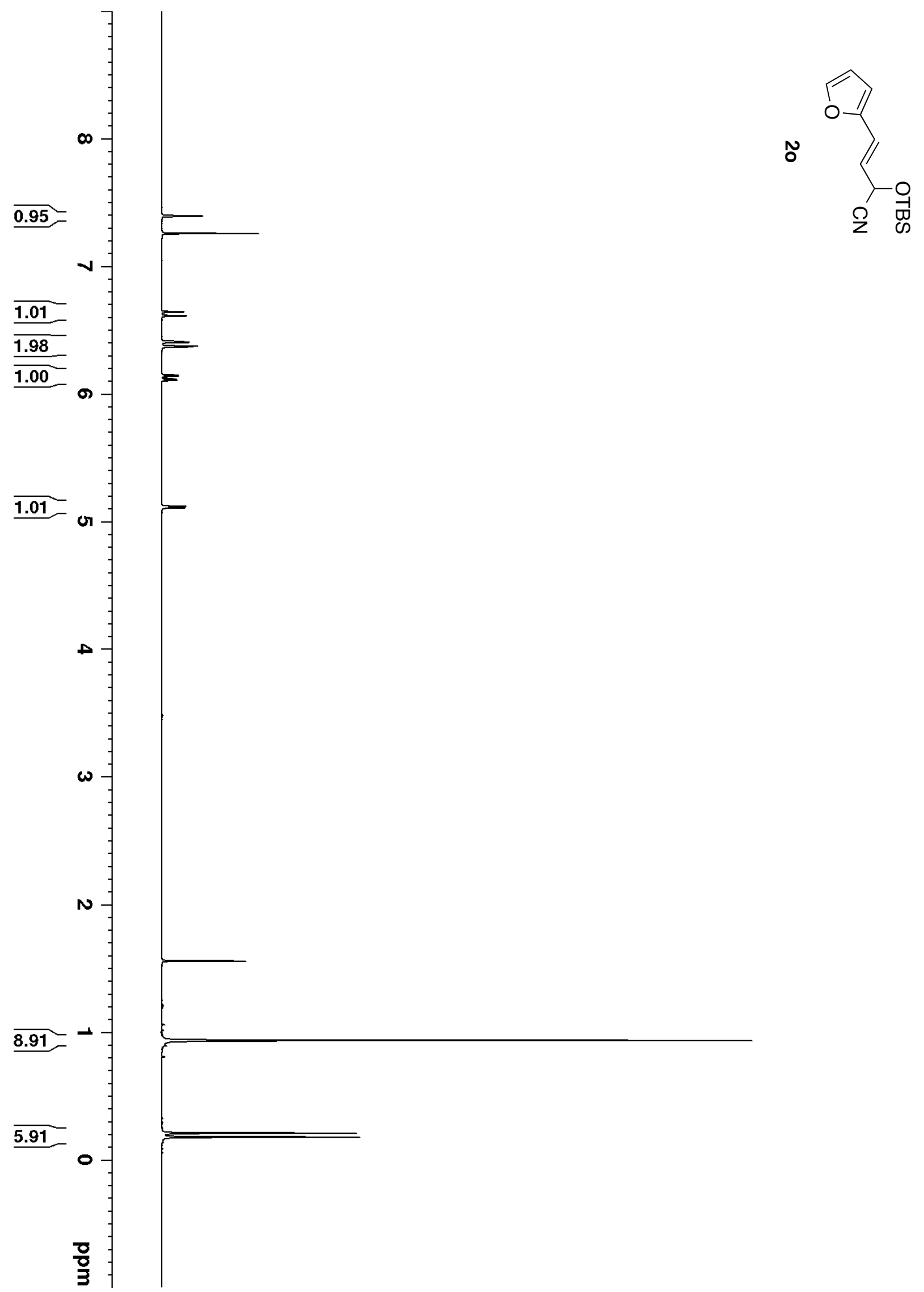


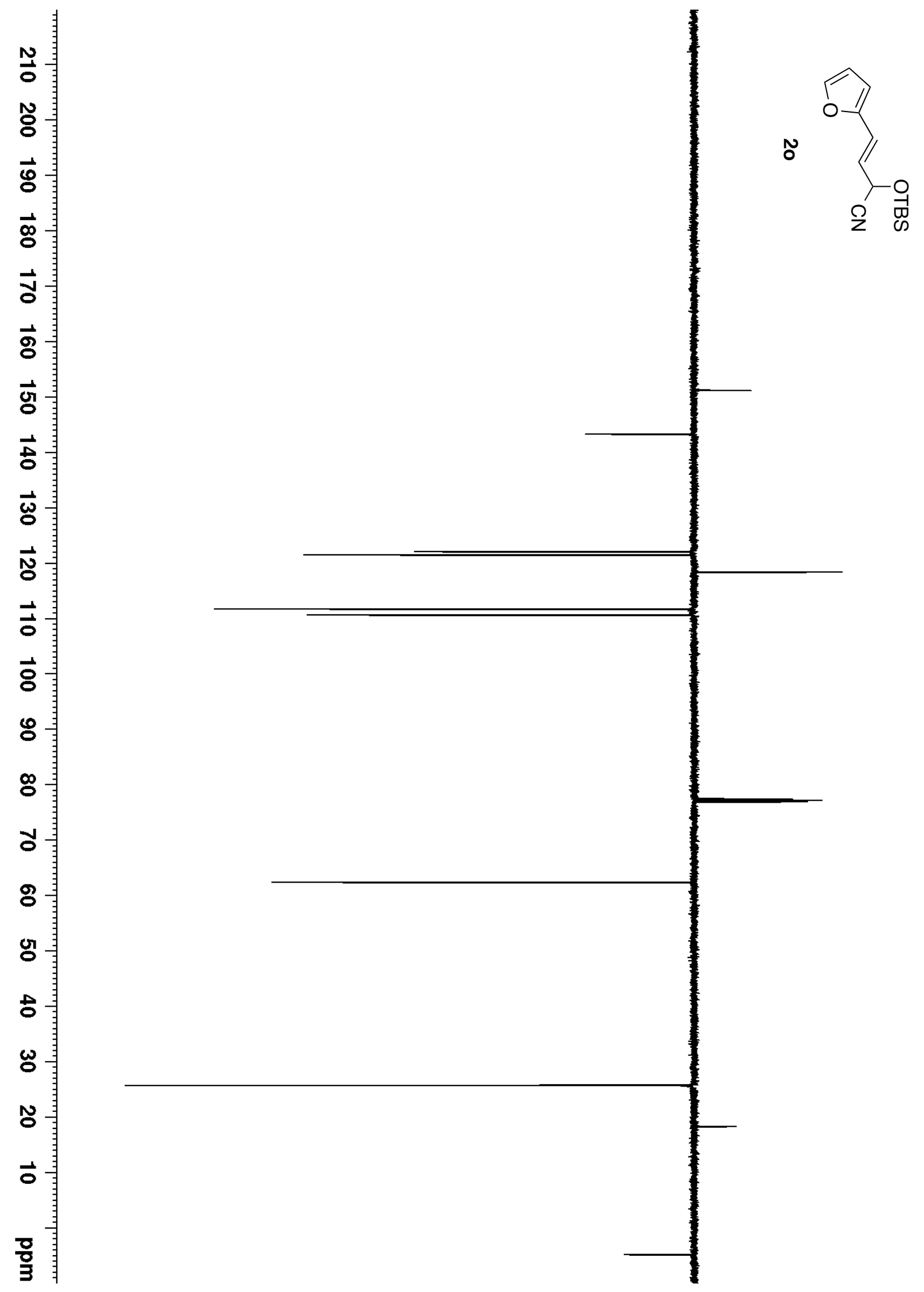




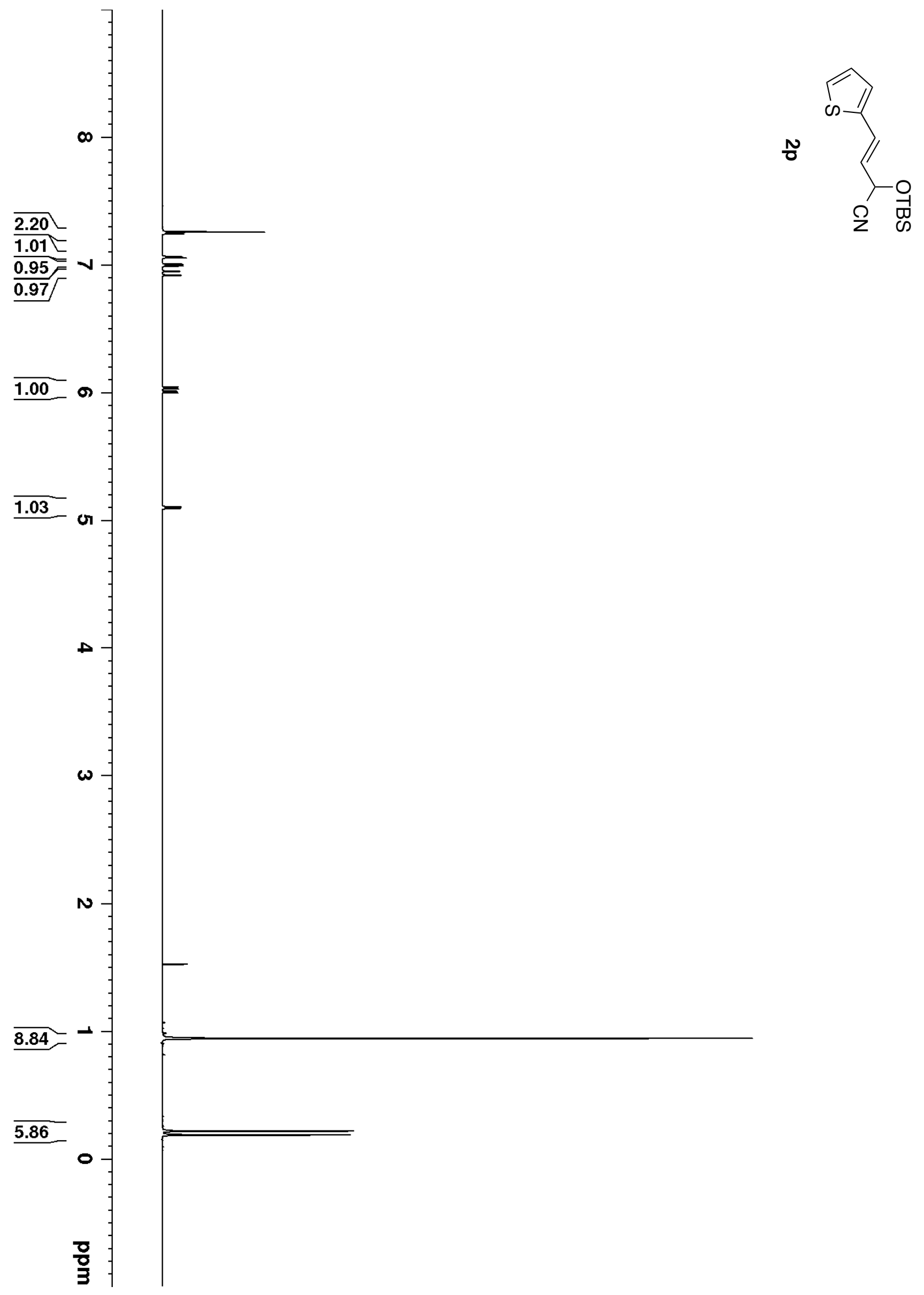




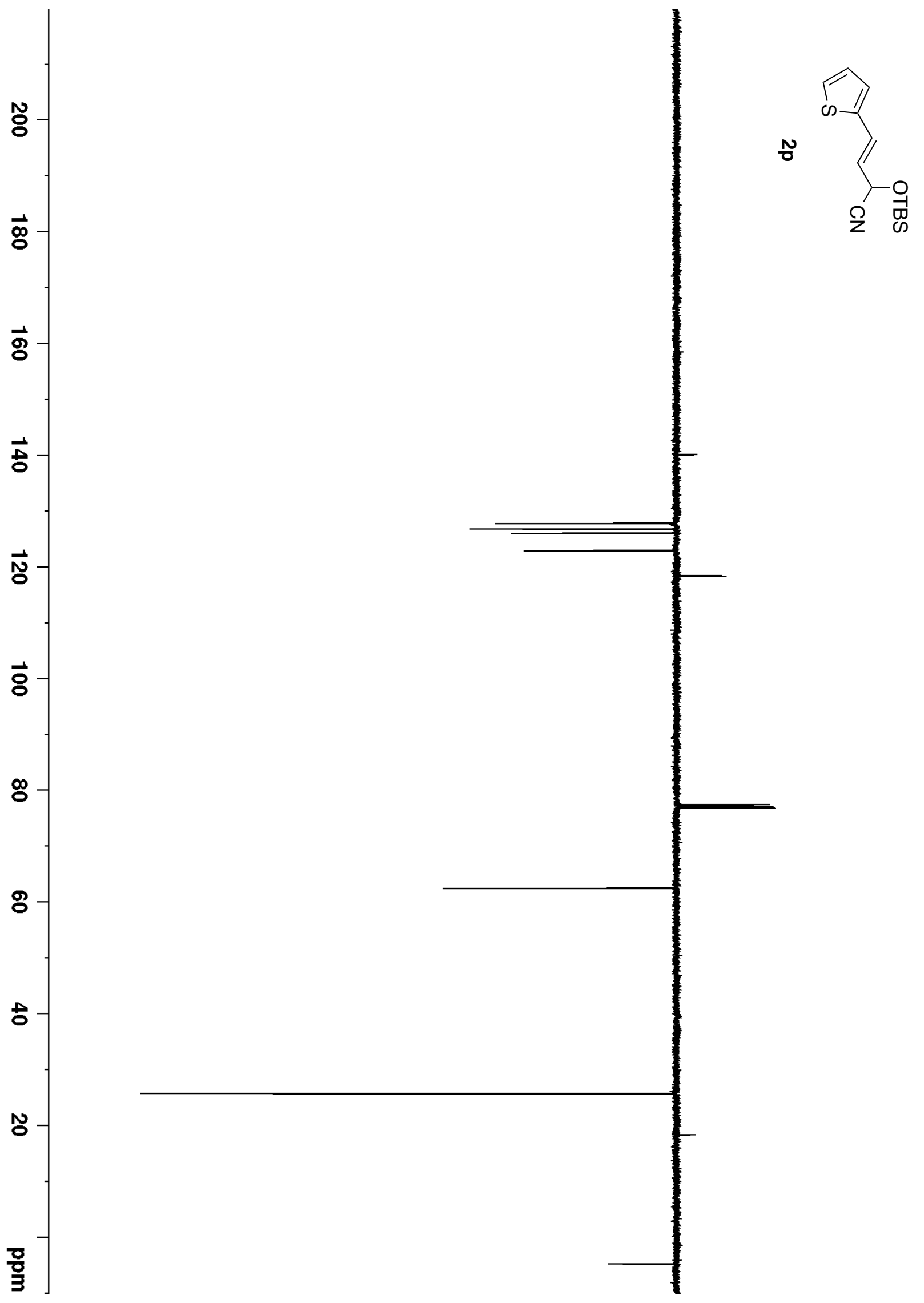




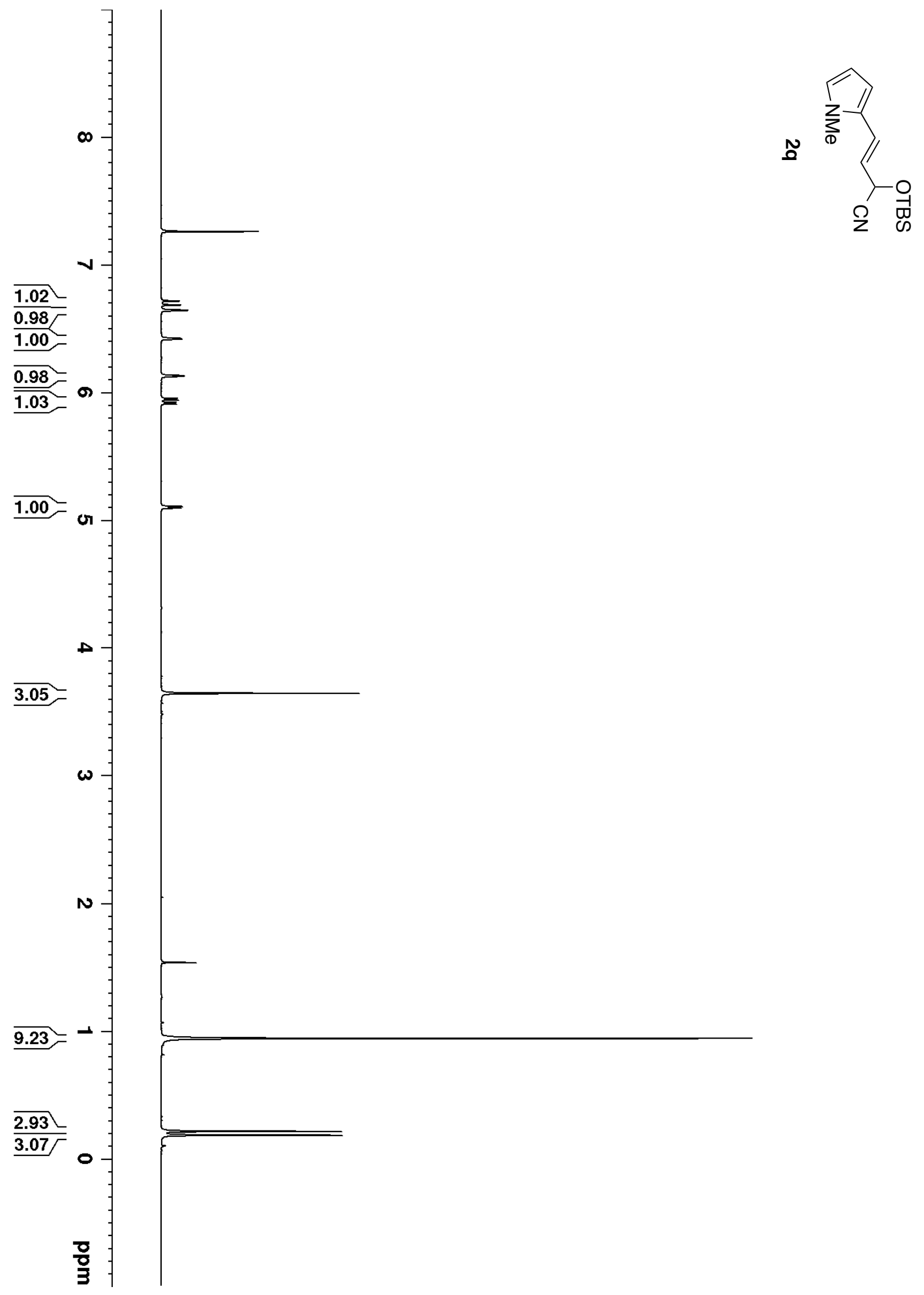




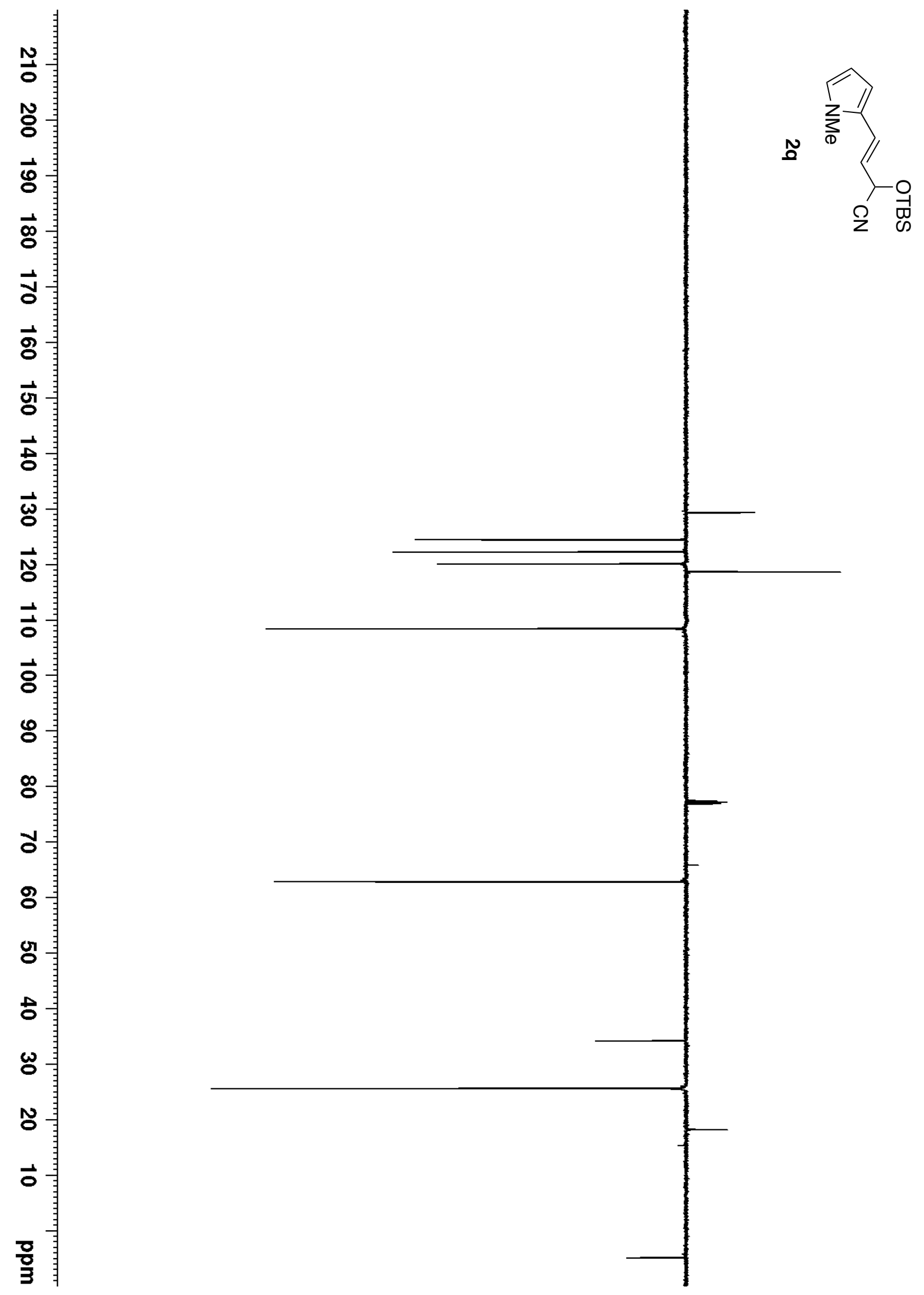



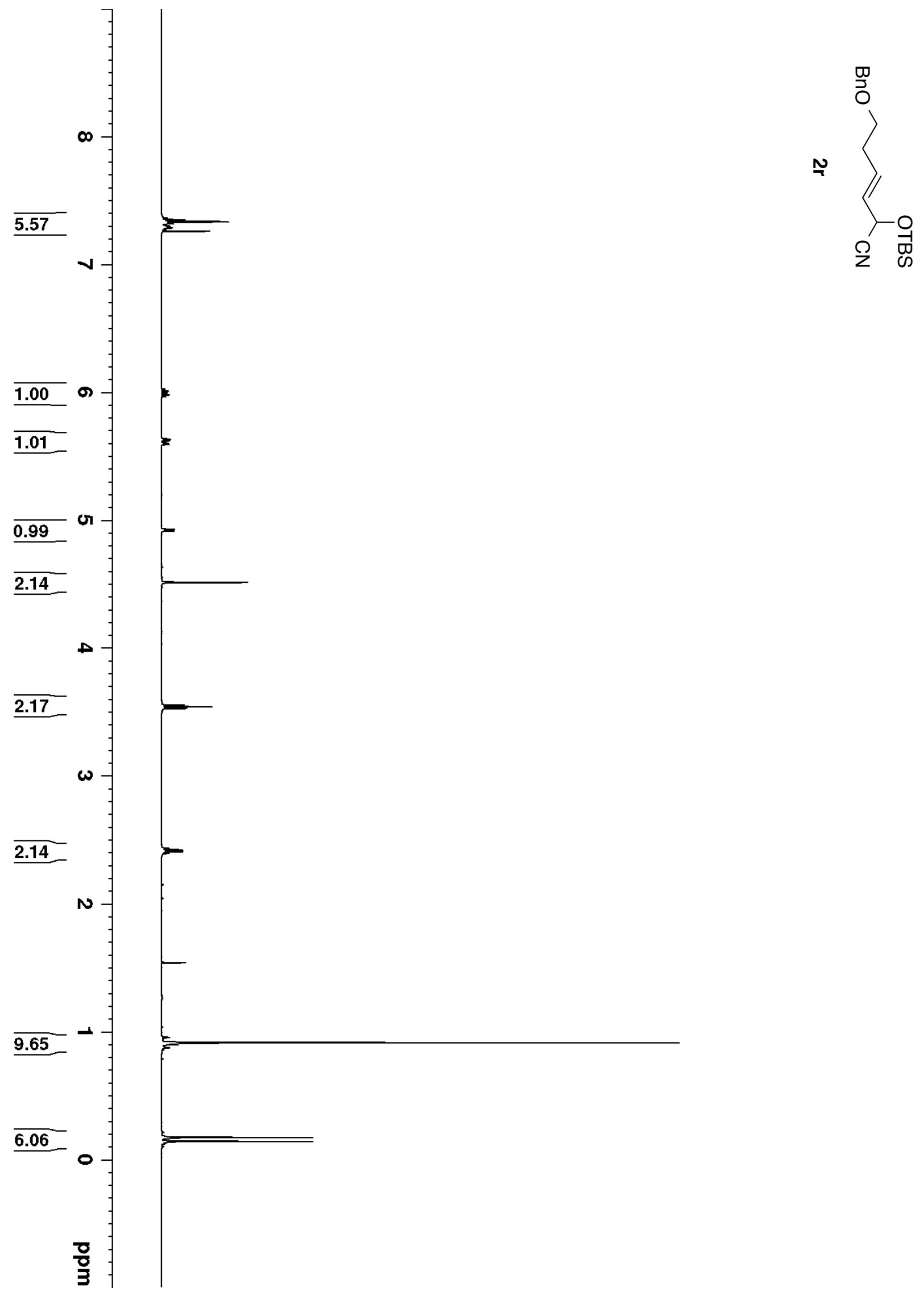


$$
H_{*}^{*}
$$




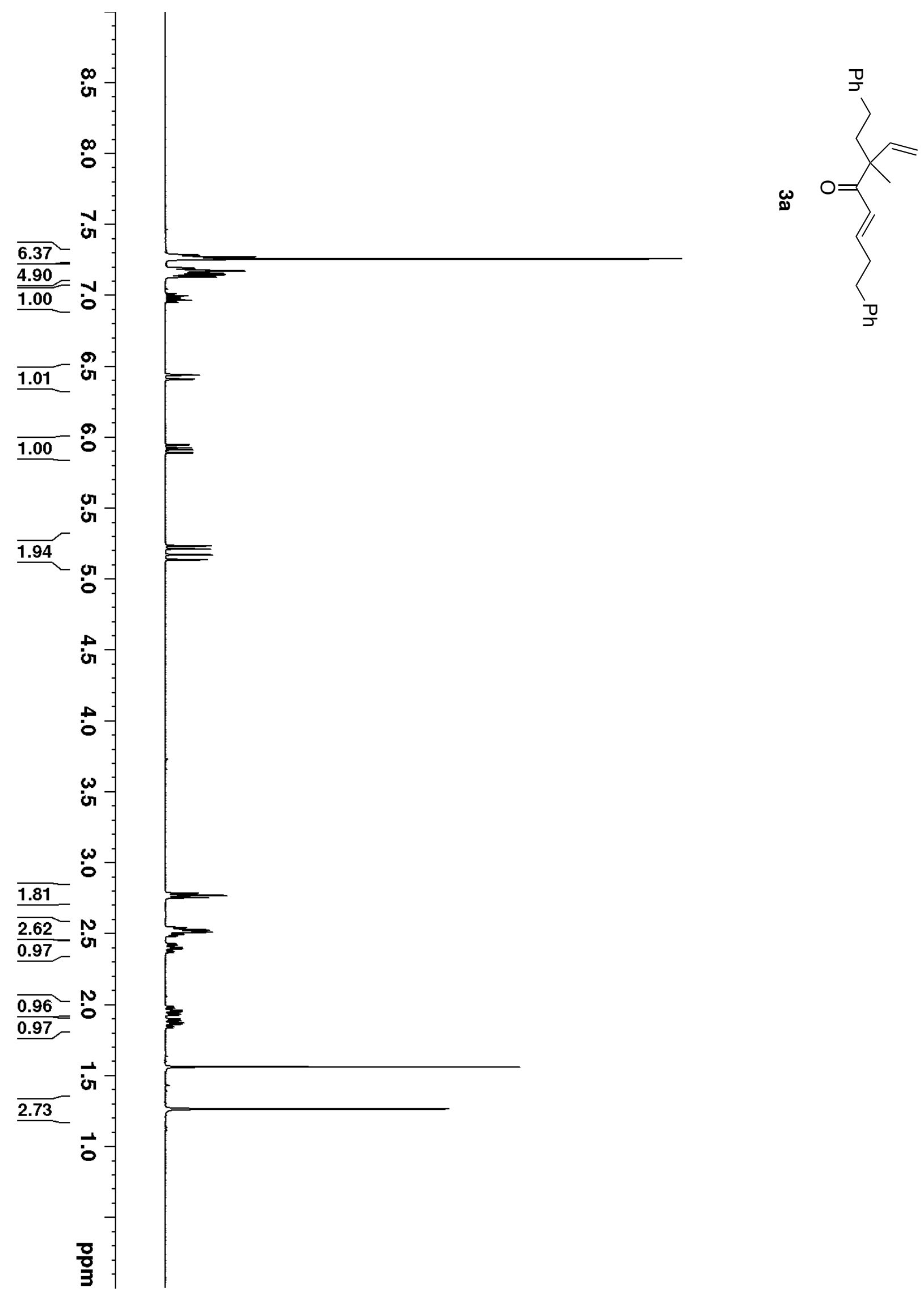




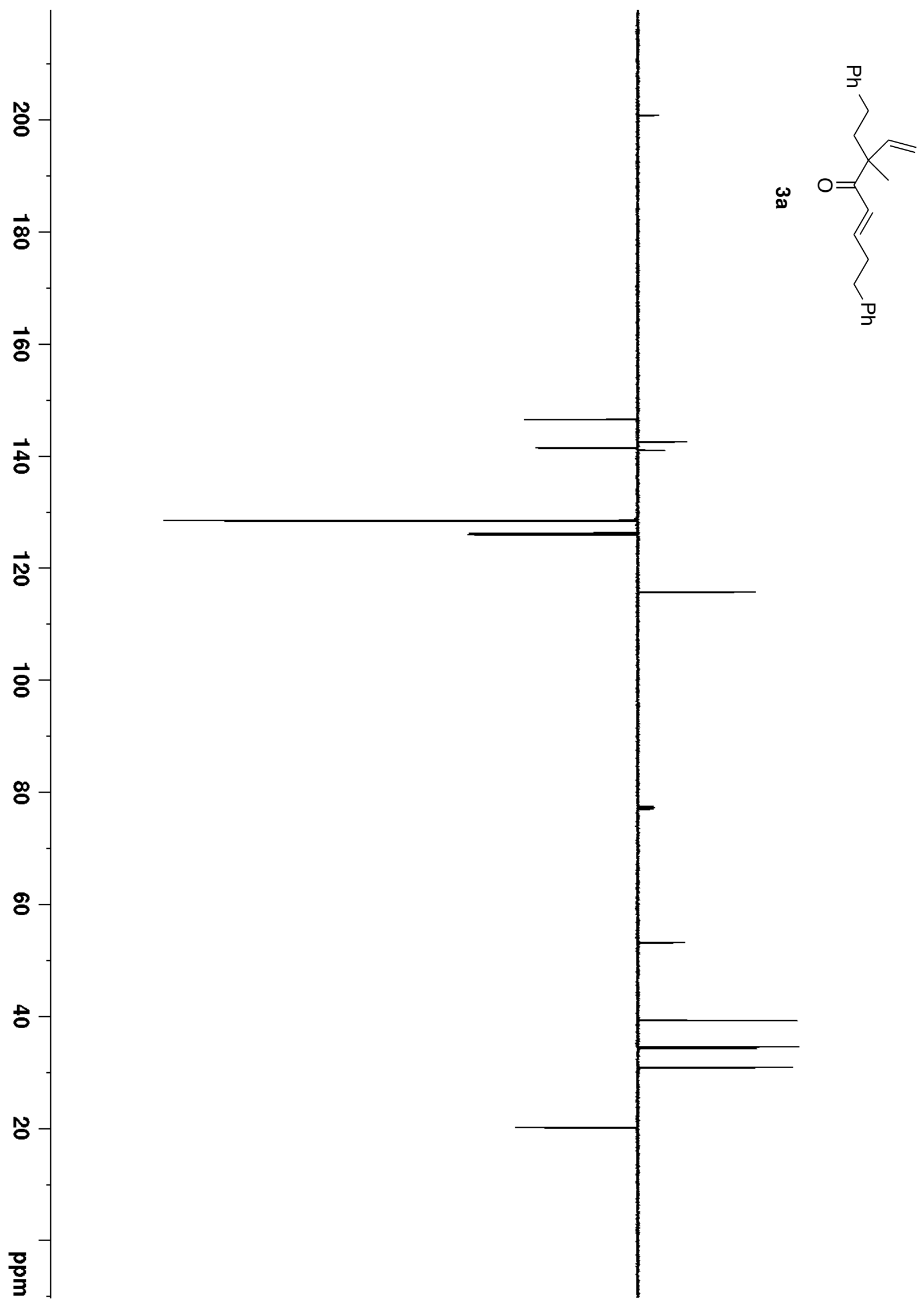



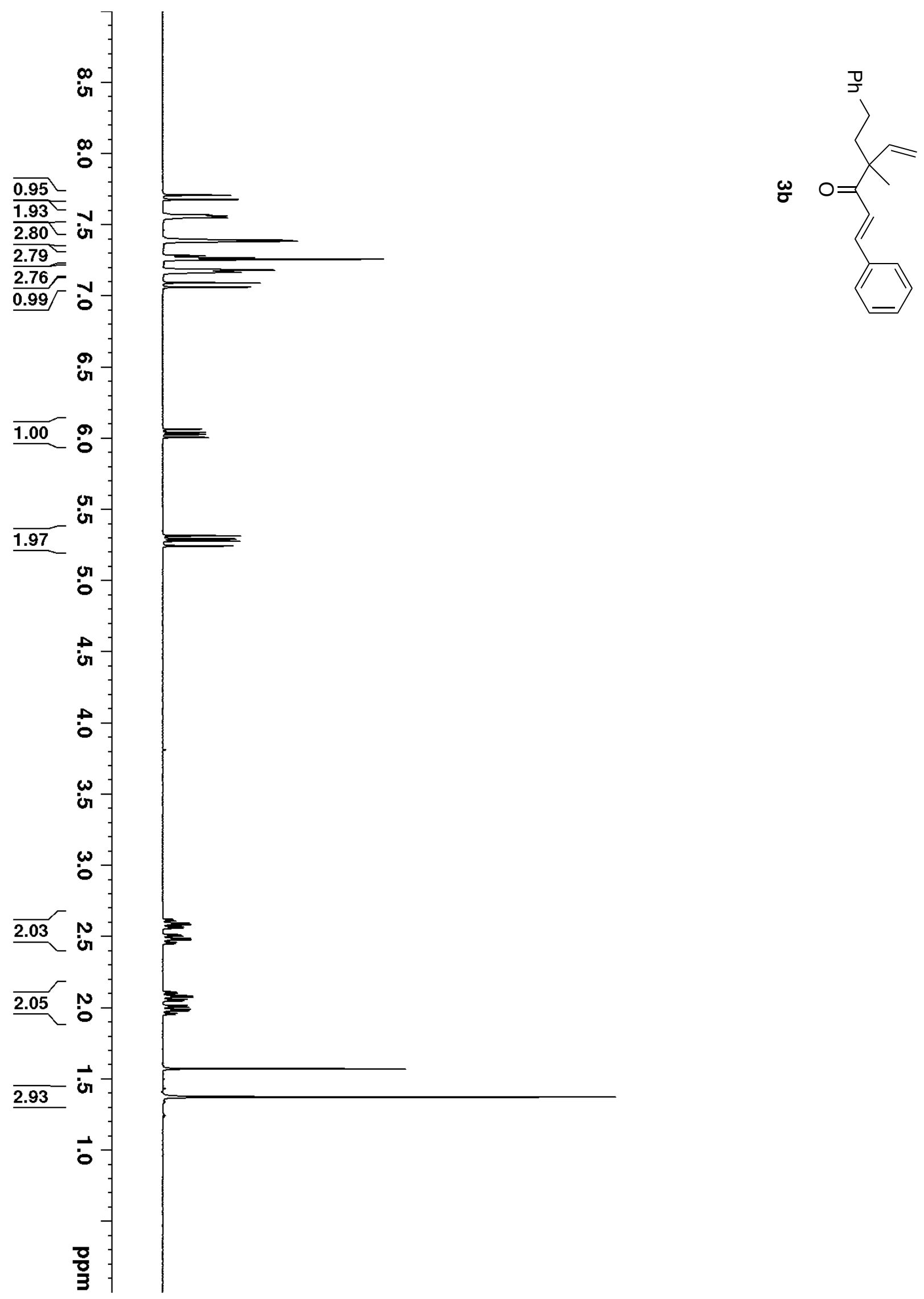


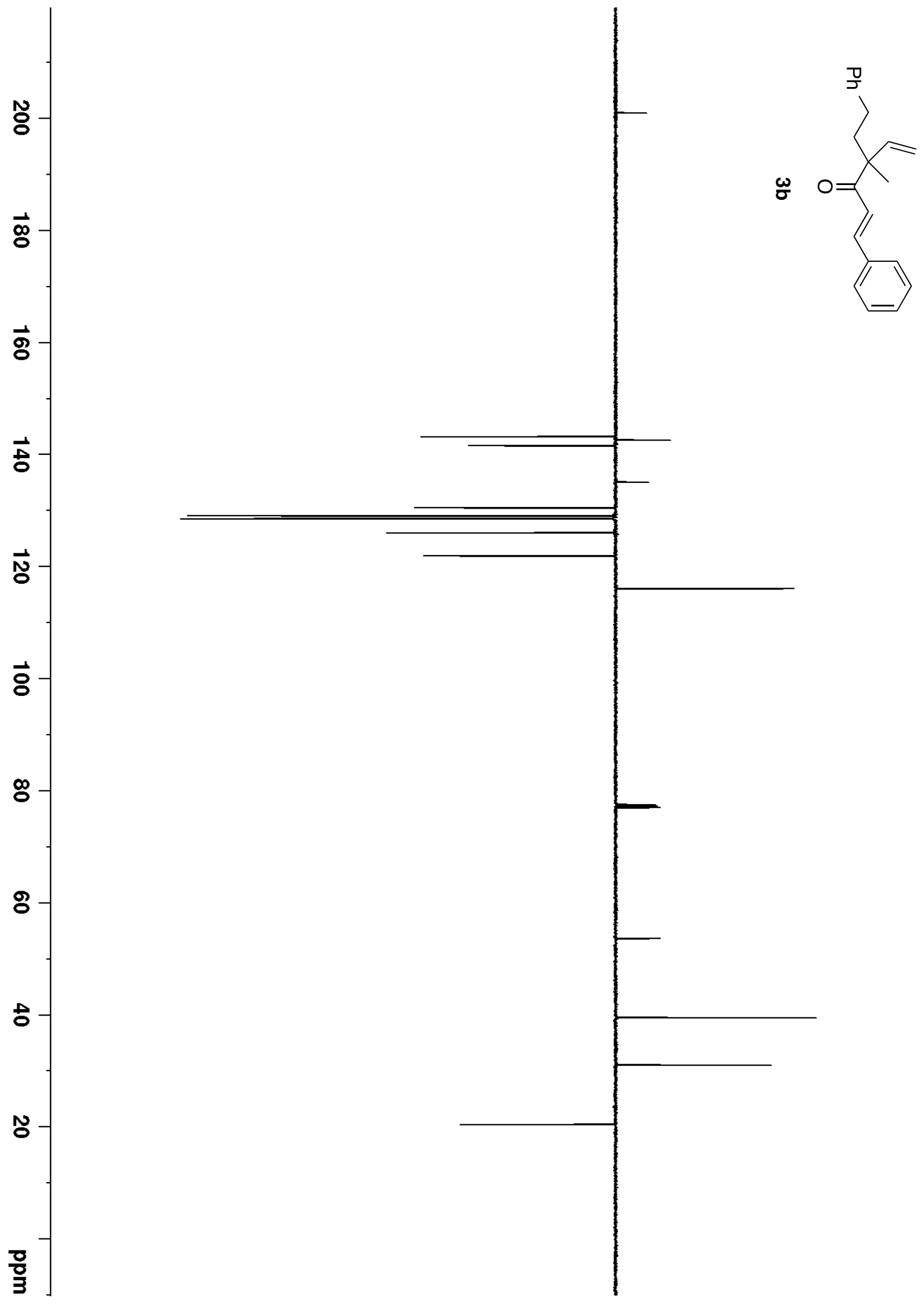




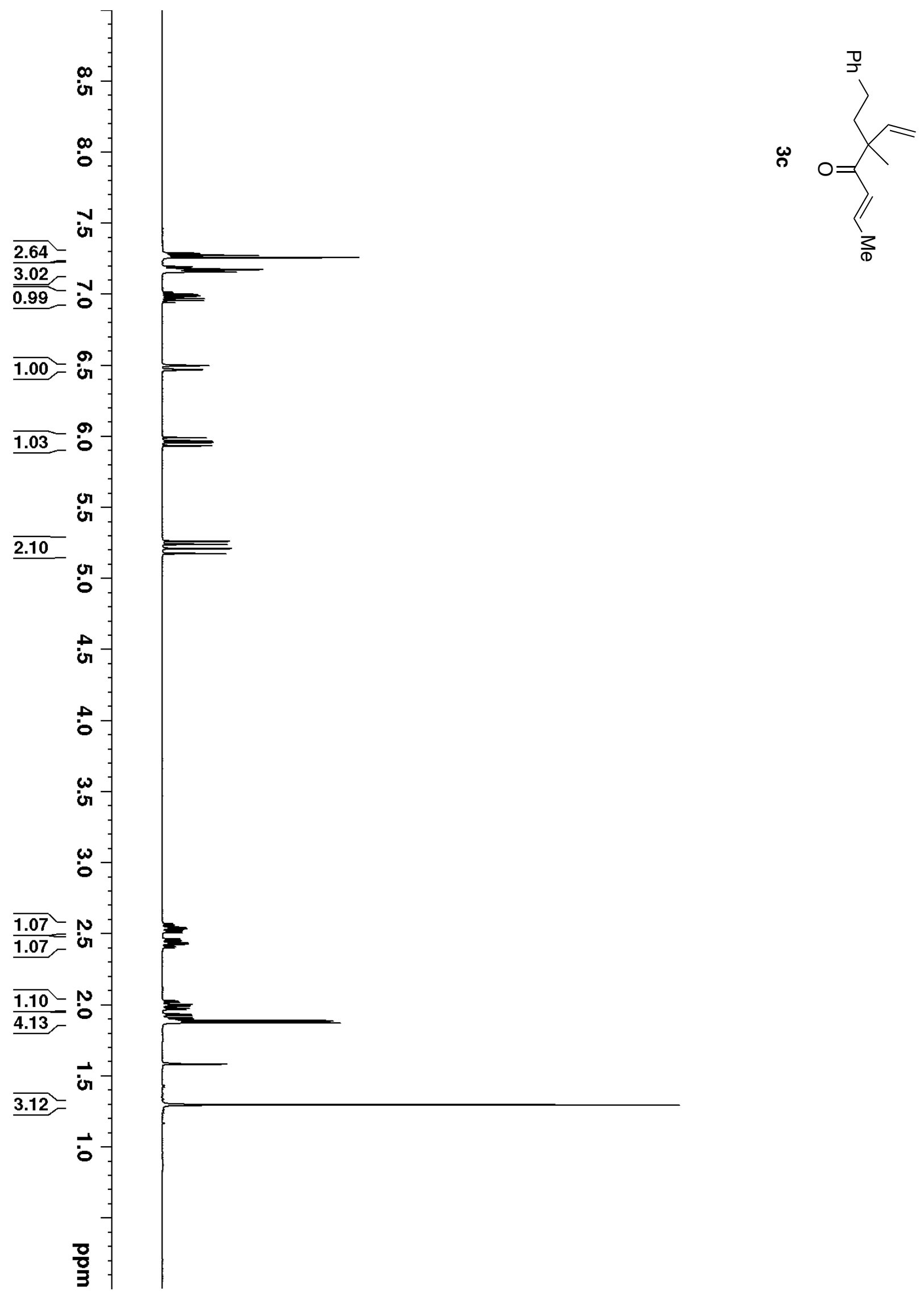




$$
1+
$$




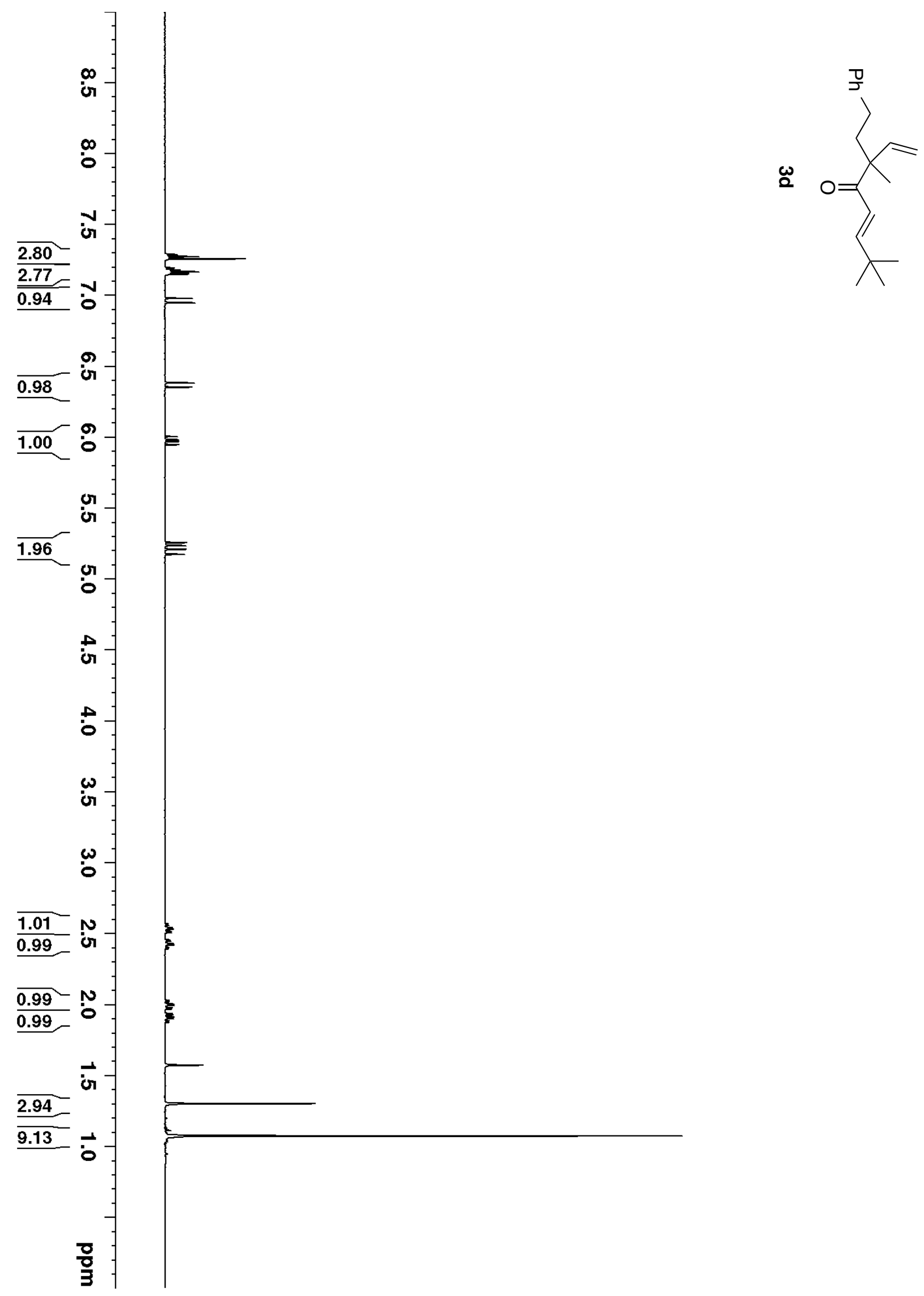




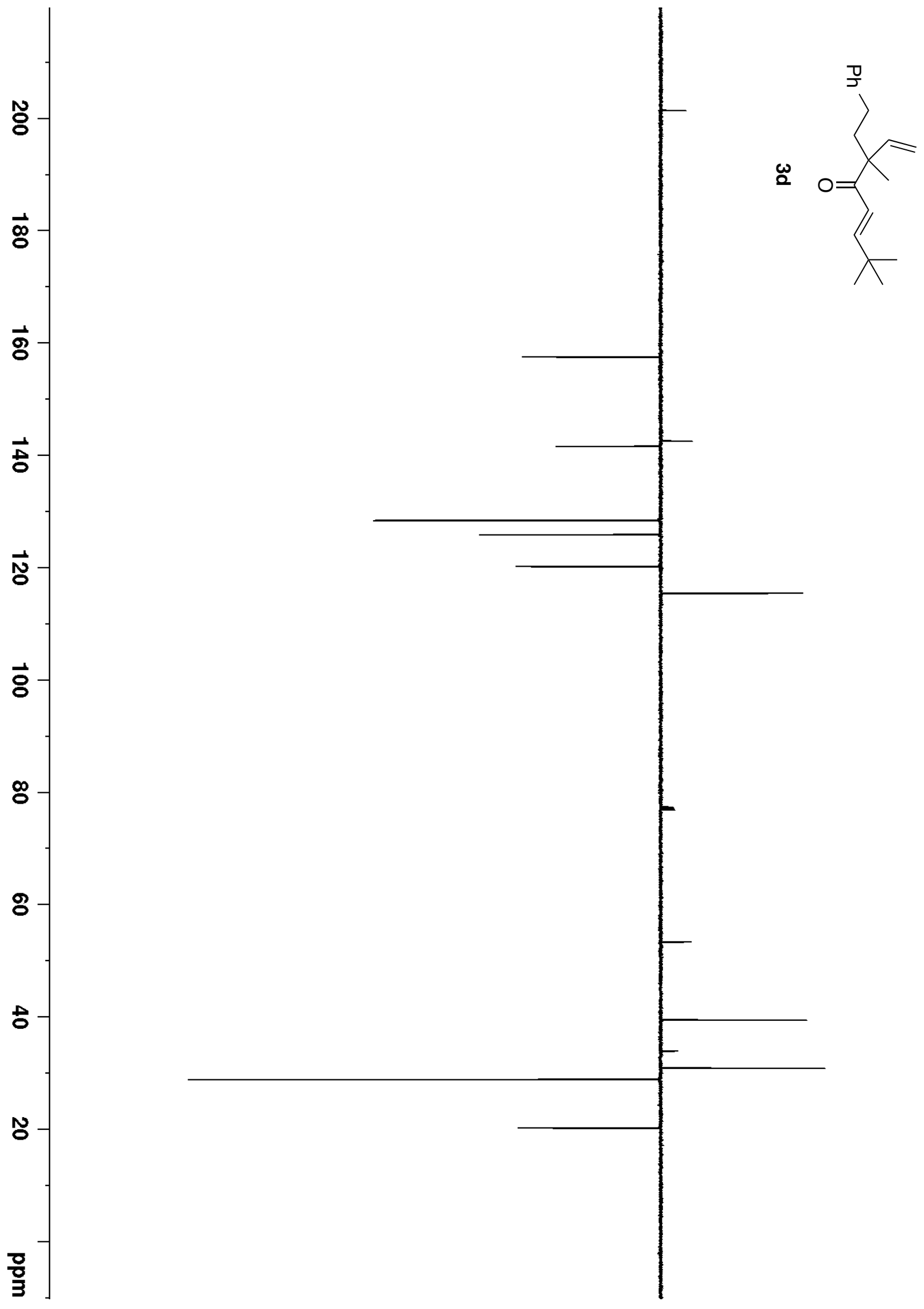




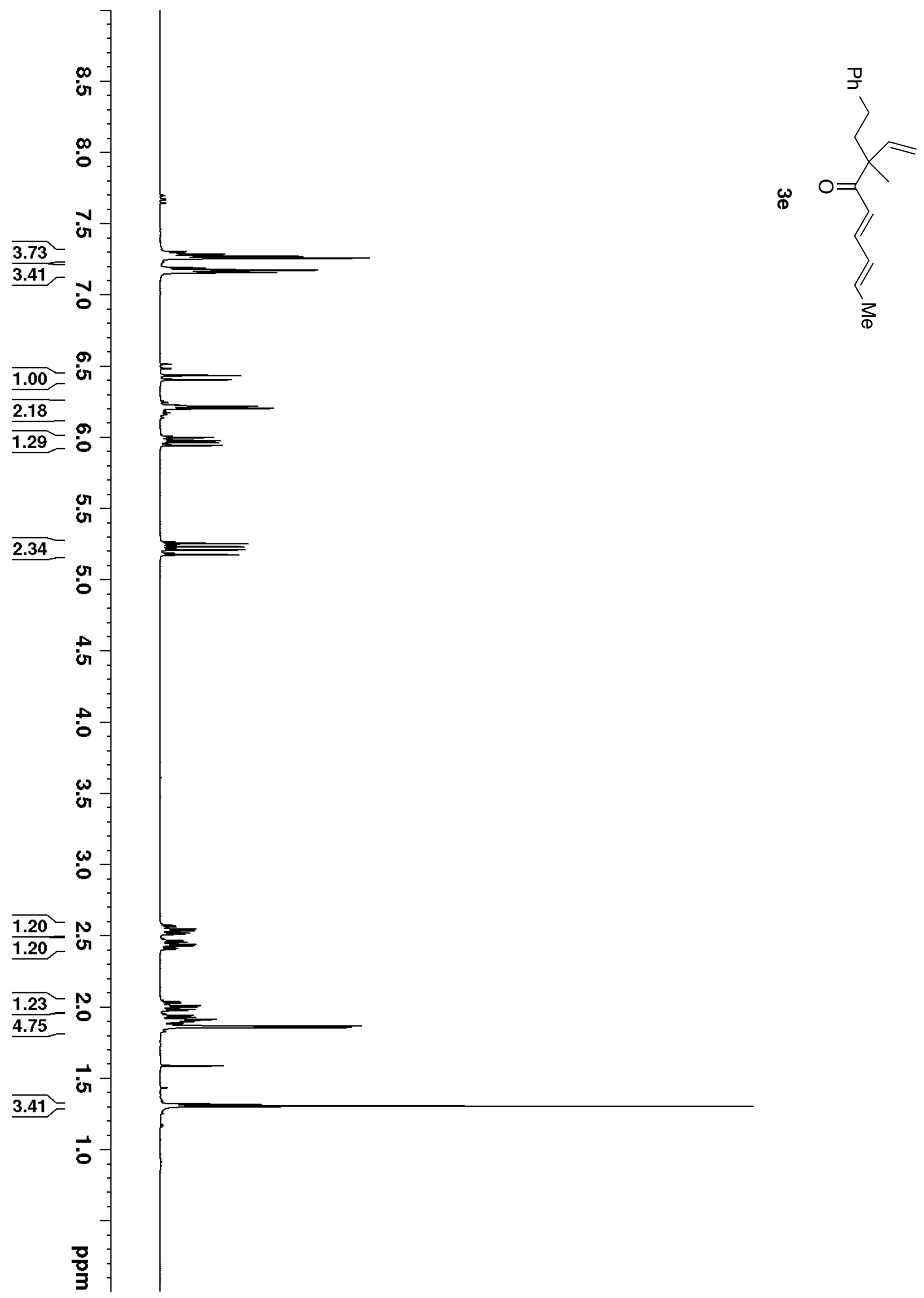




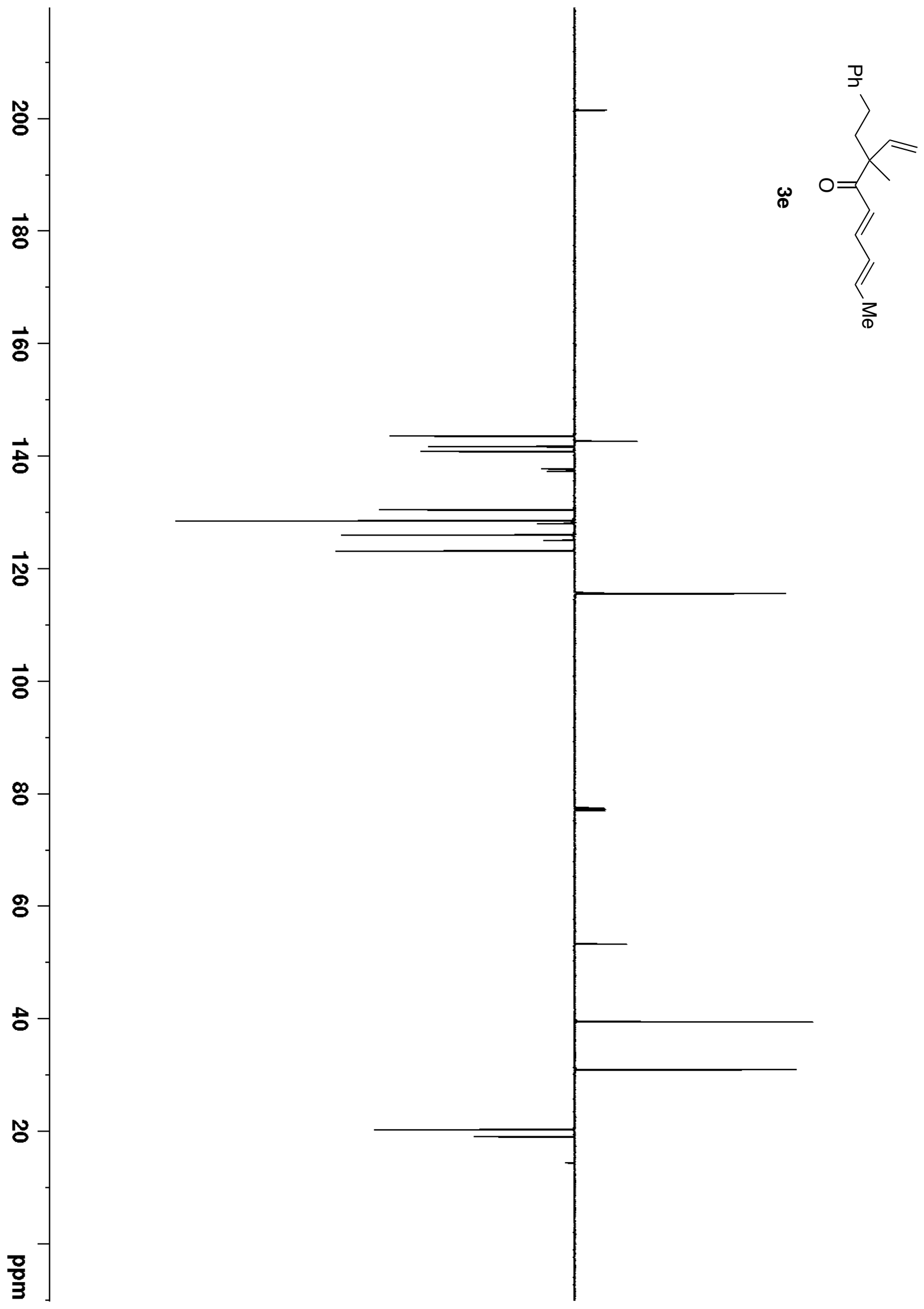



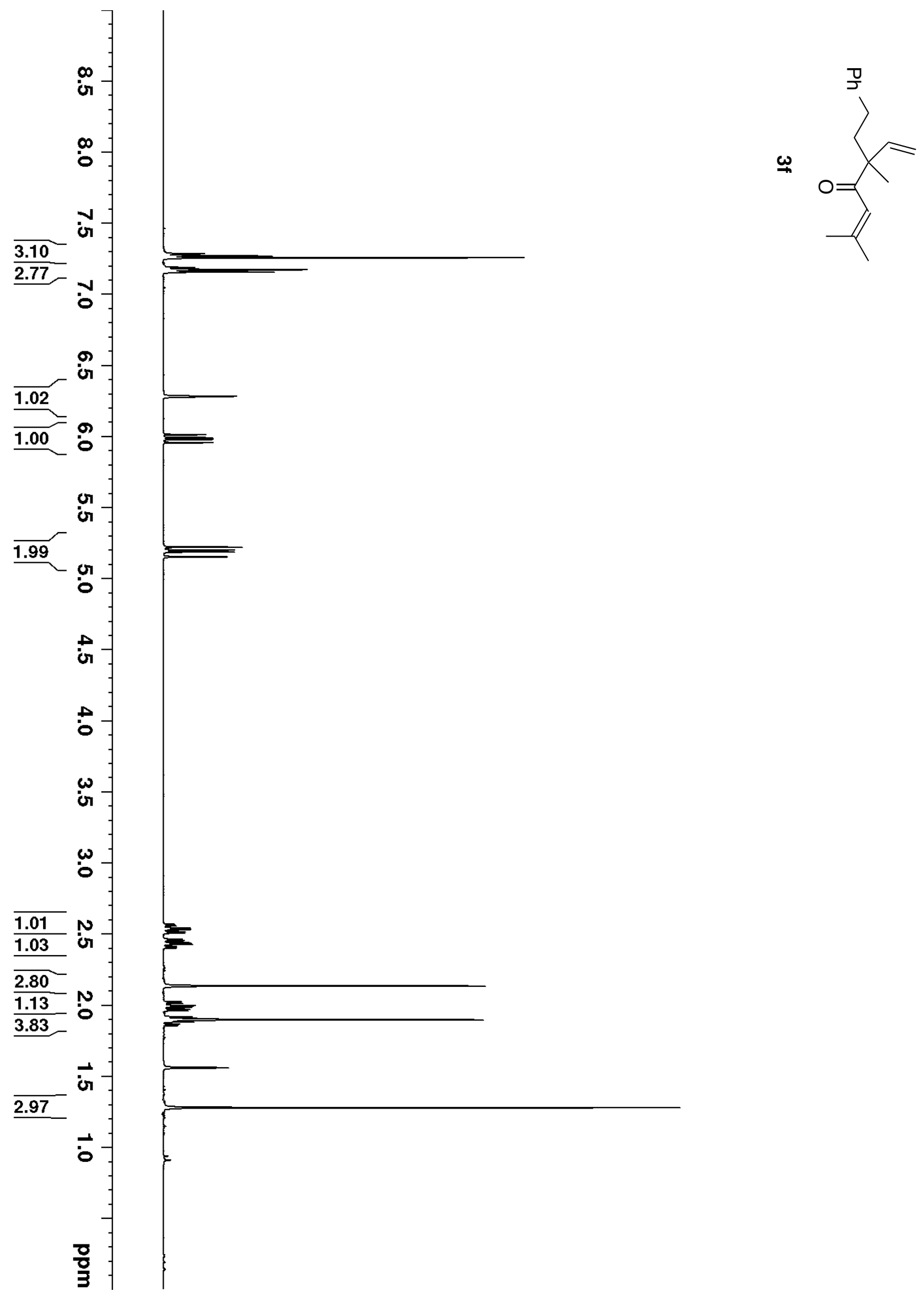


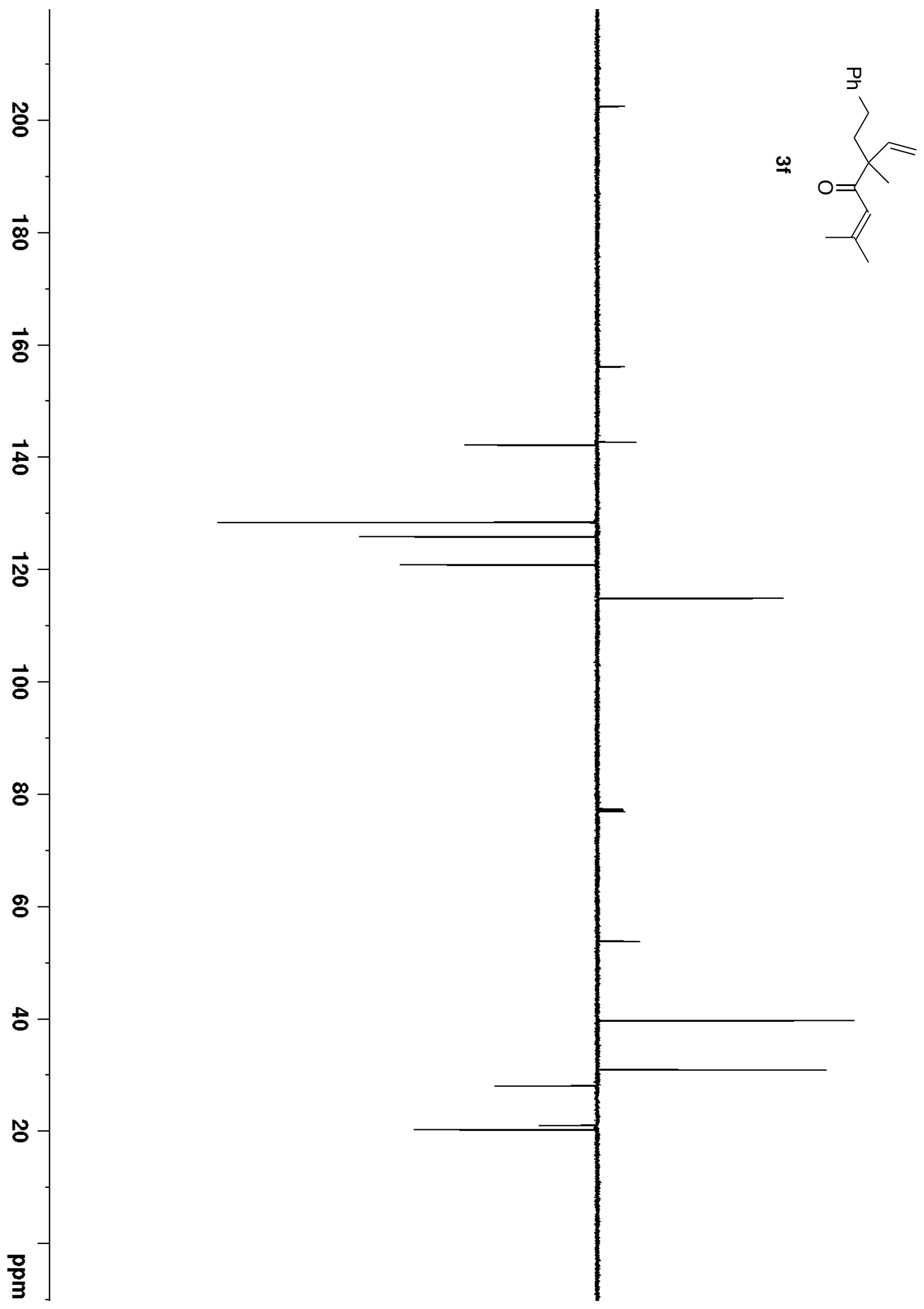




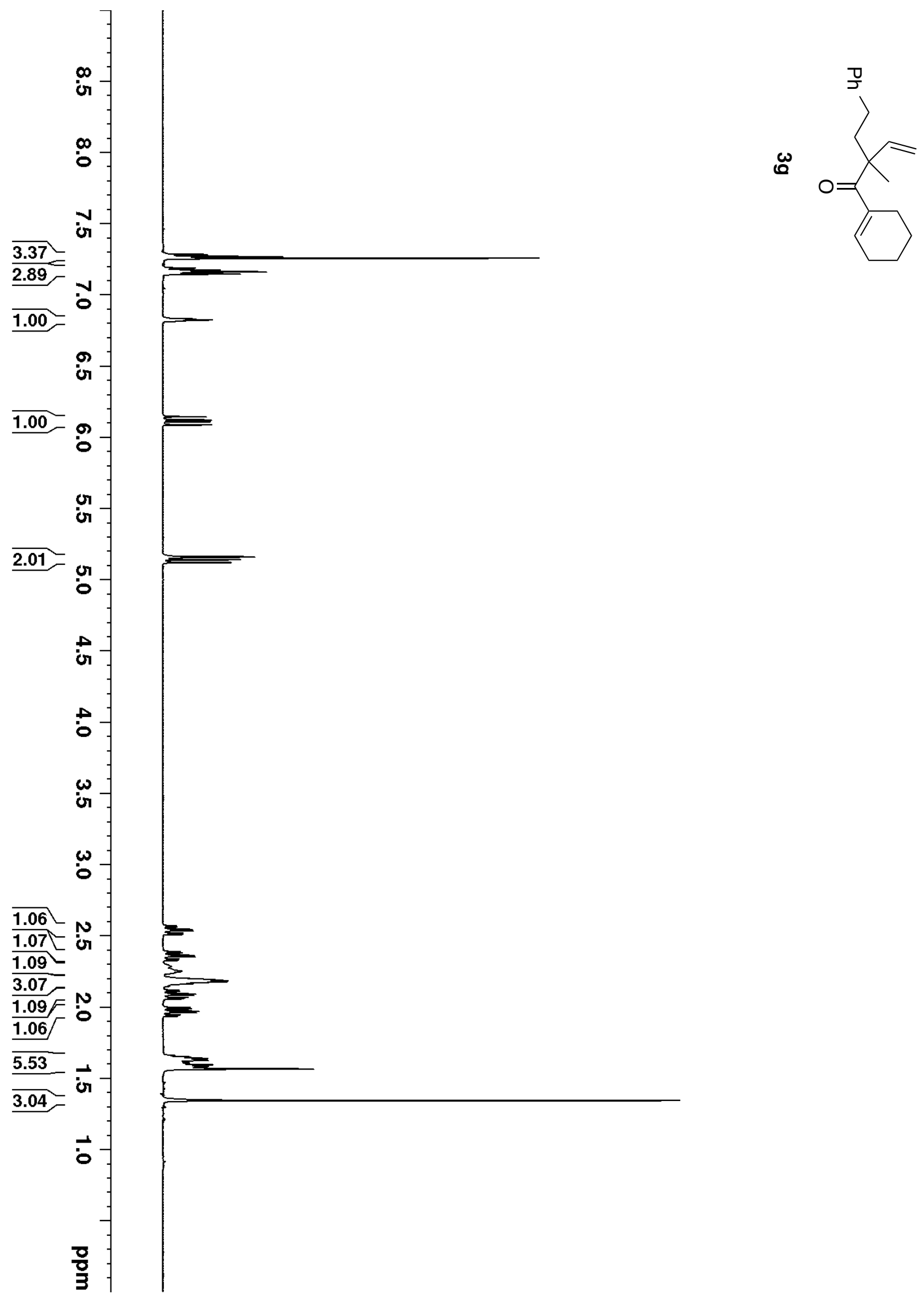




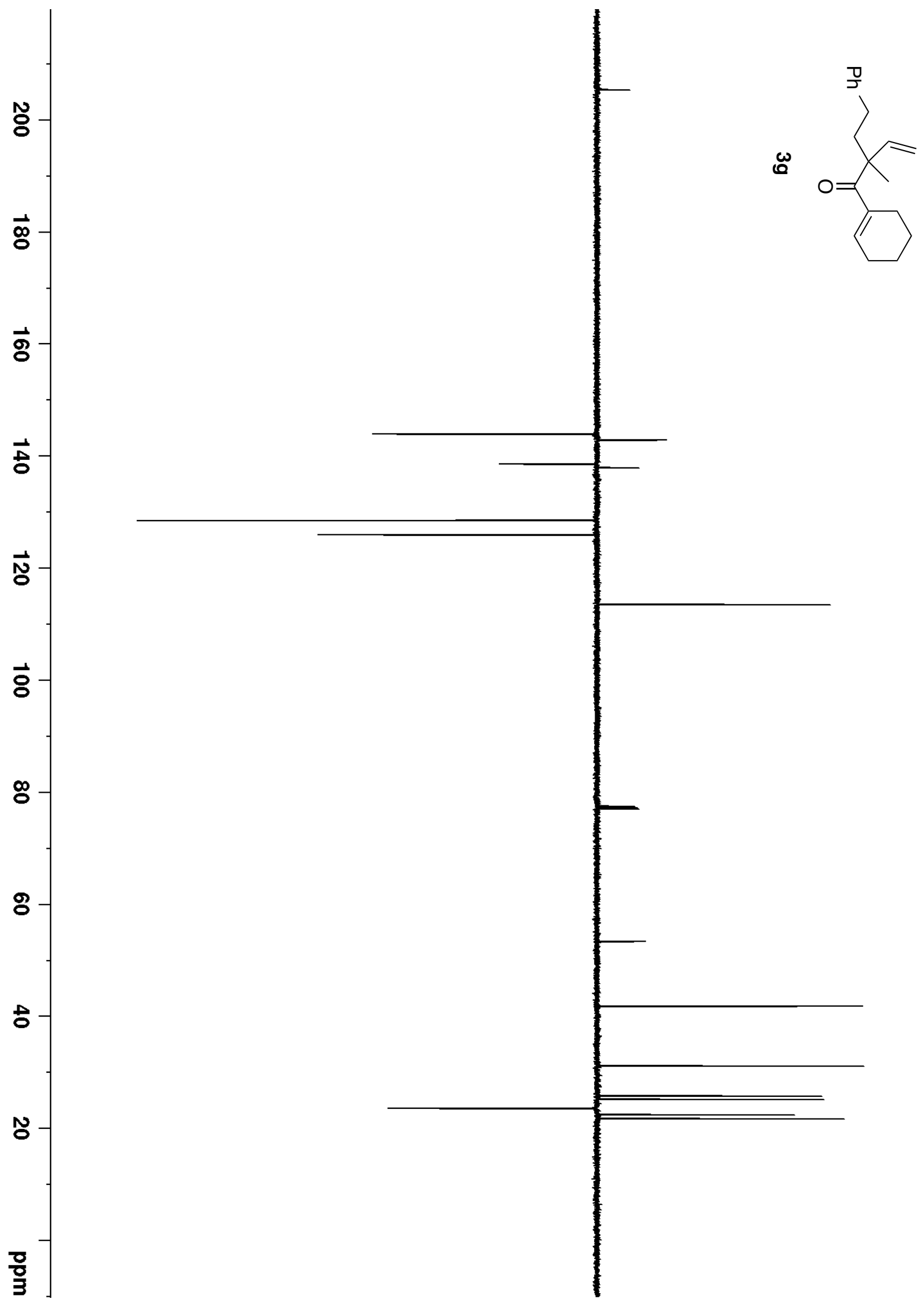




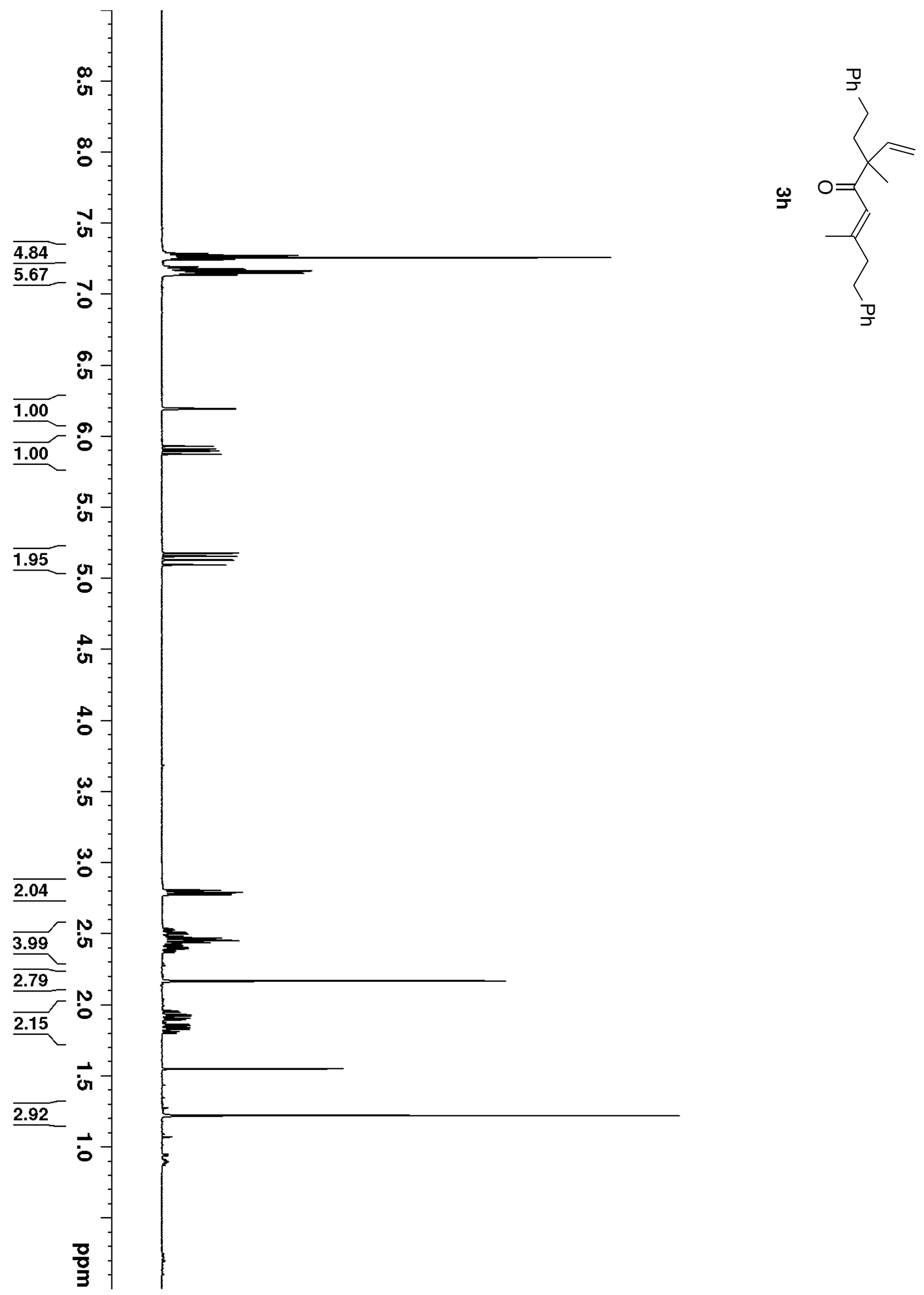




$$
1+
$$




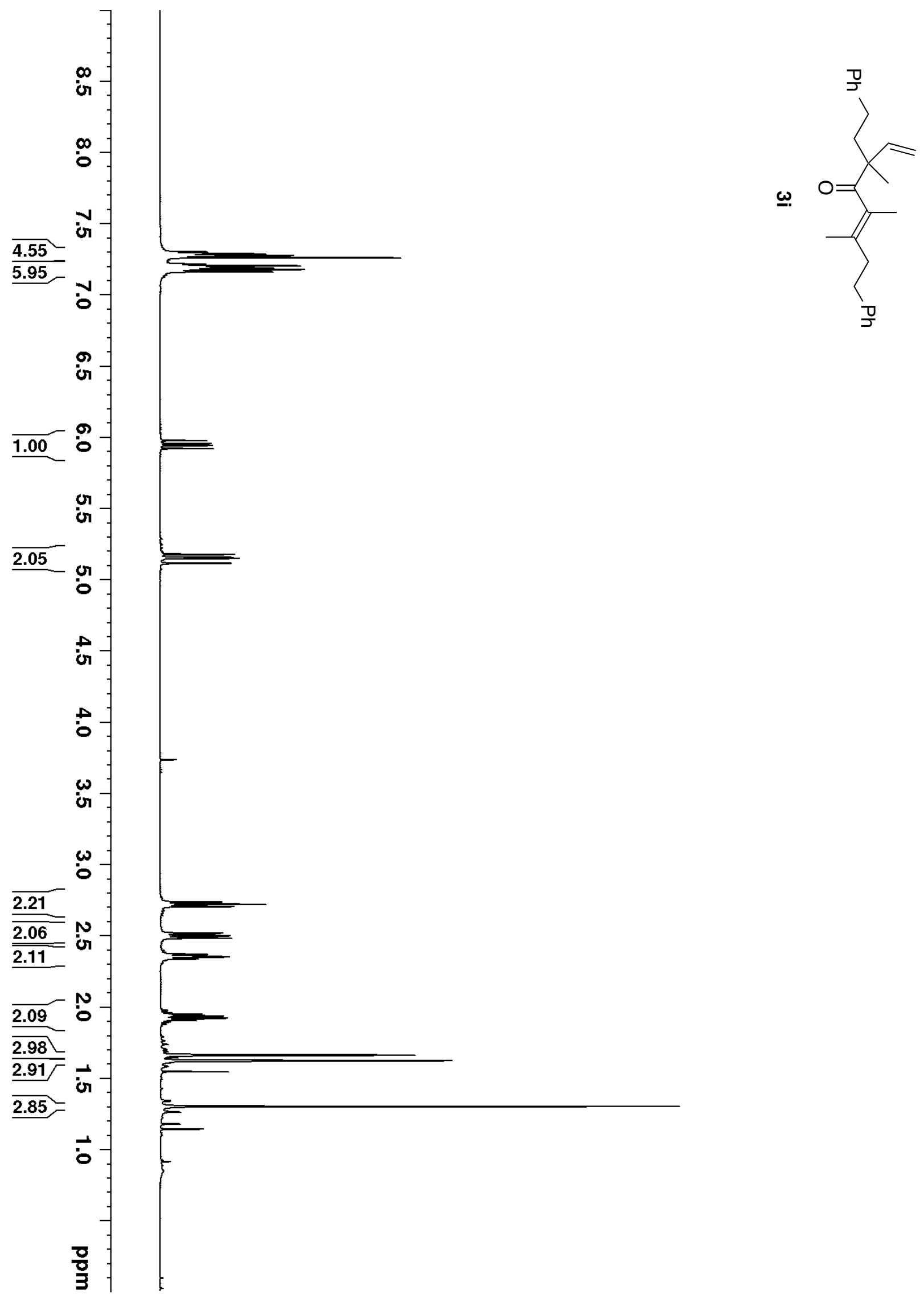




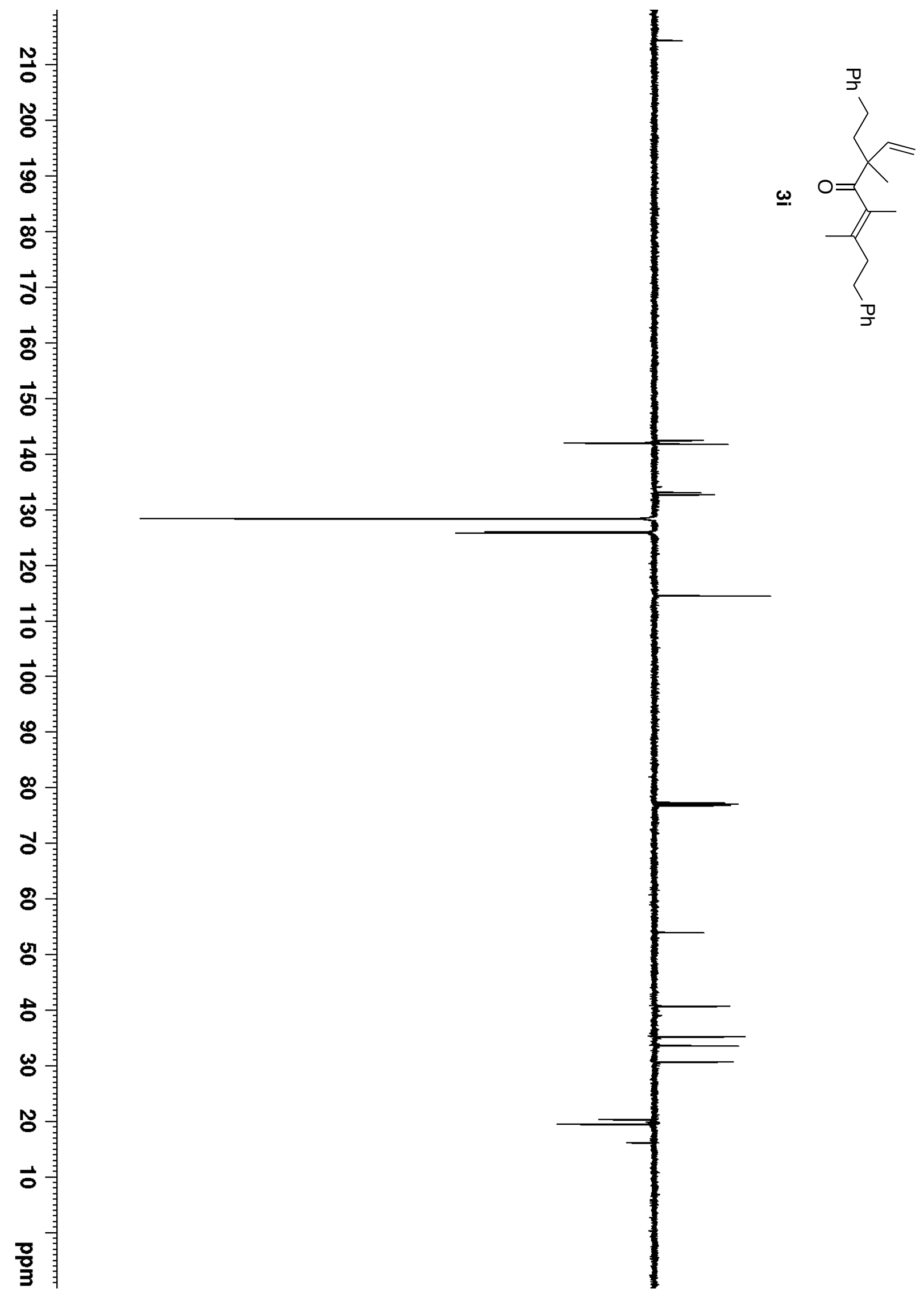




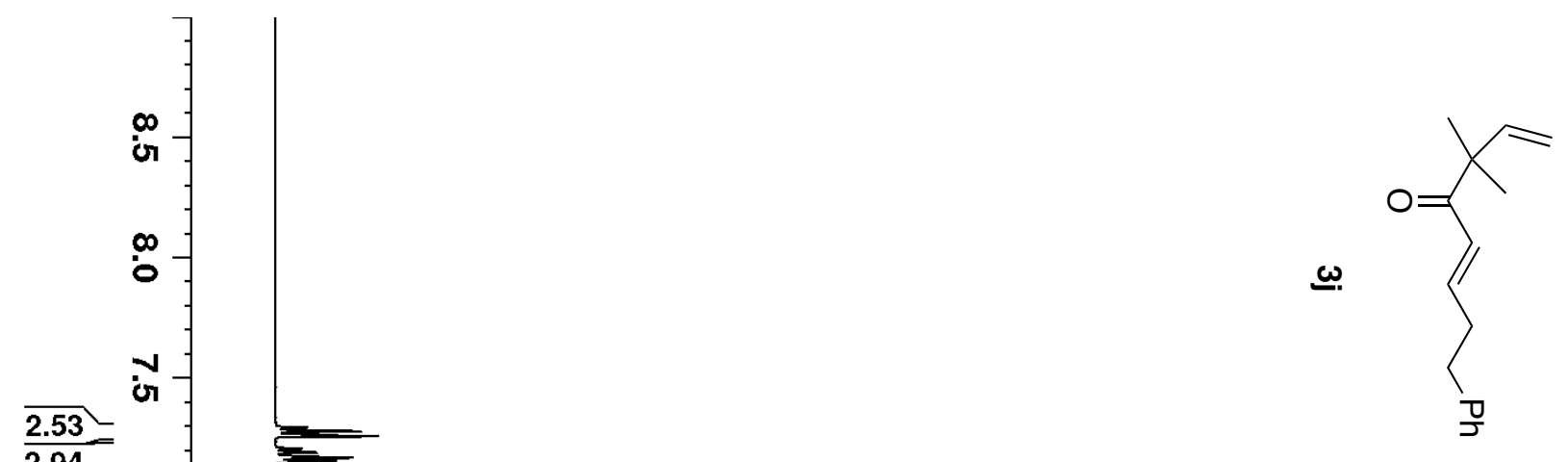




$$
\text { 柆 }
$$




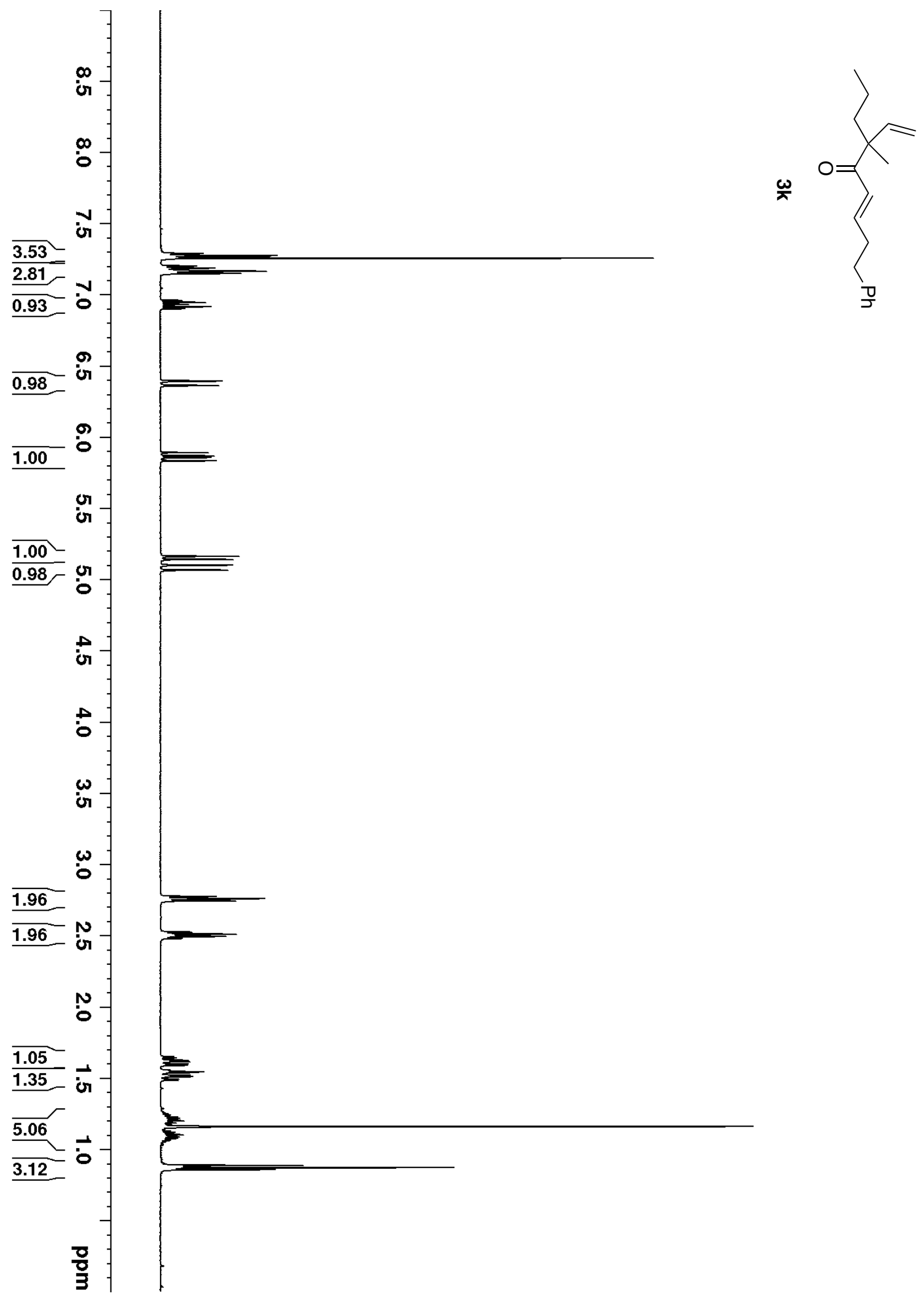




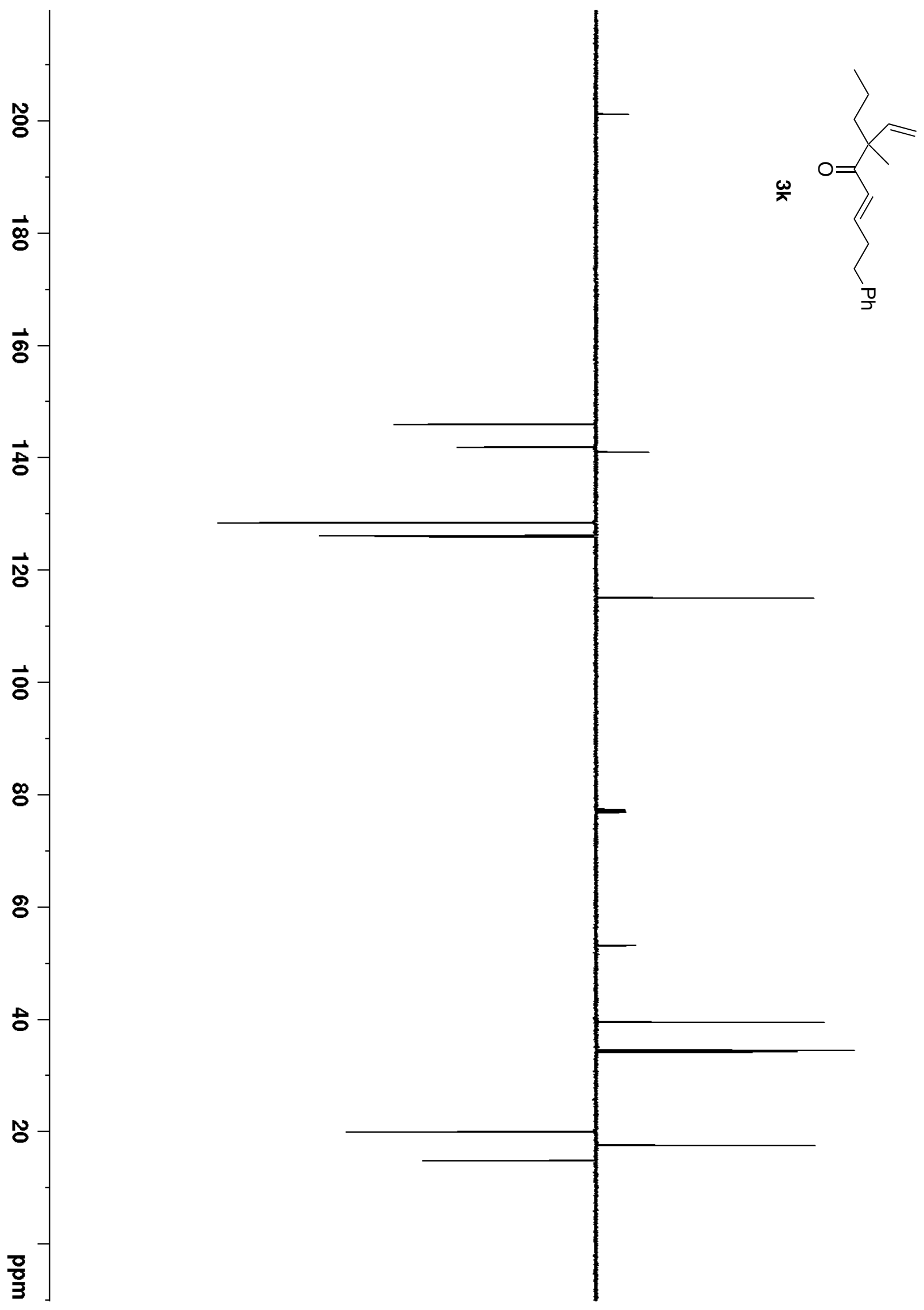




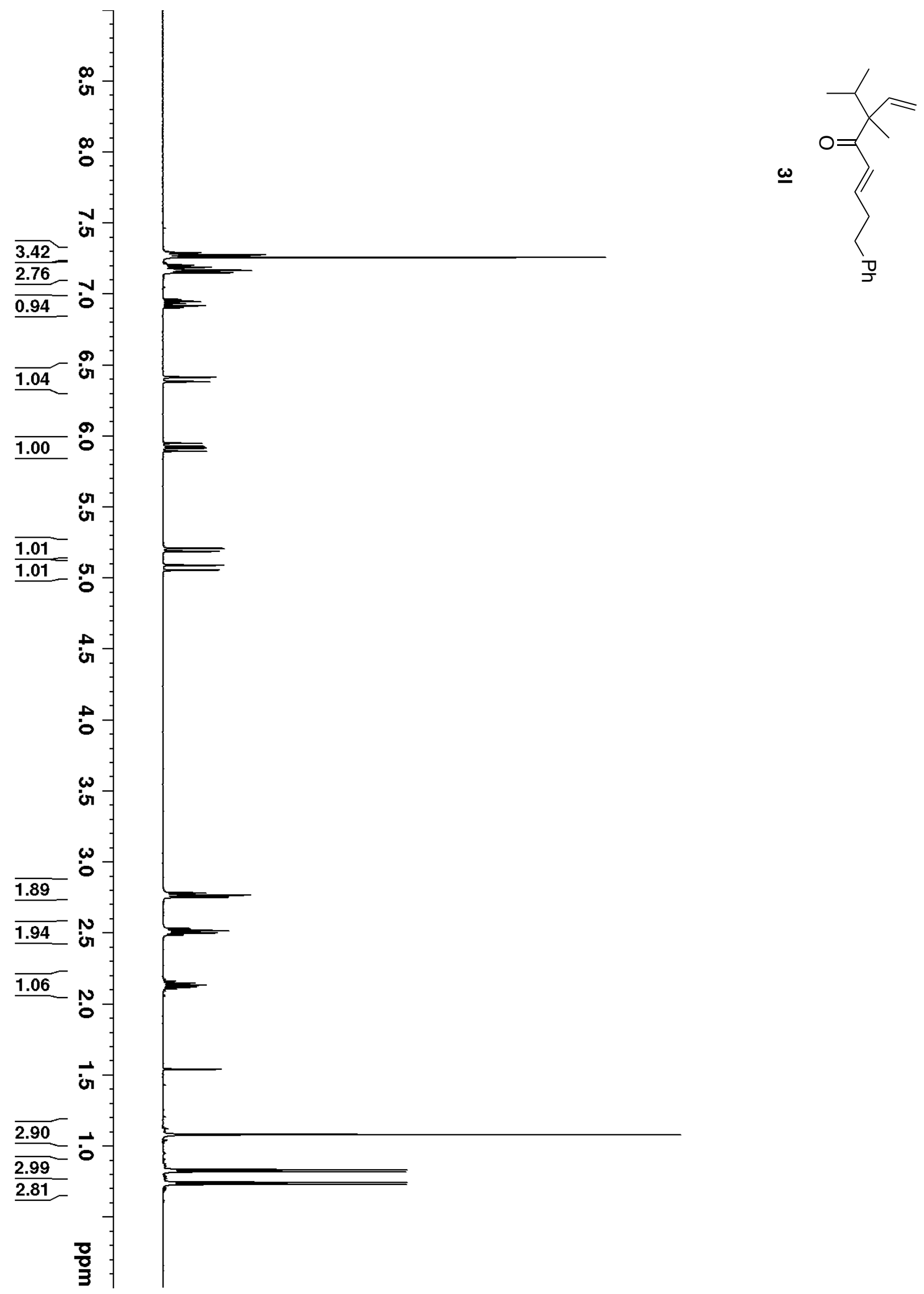



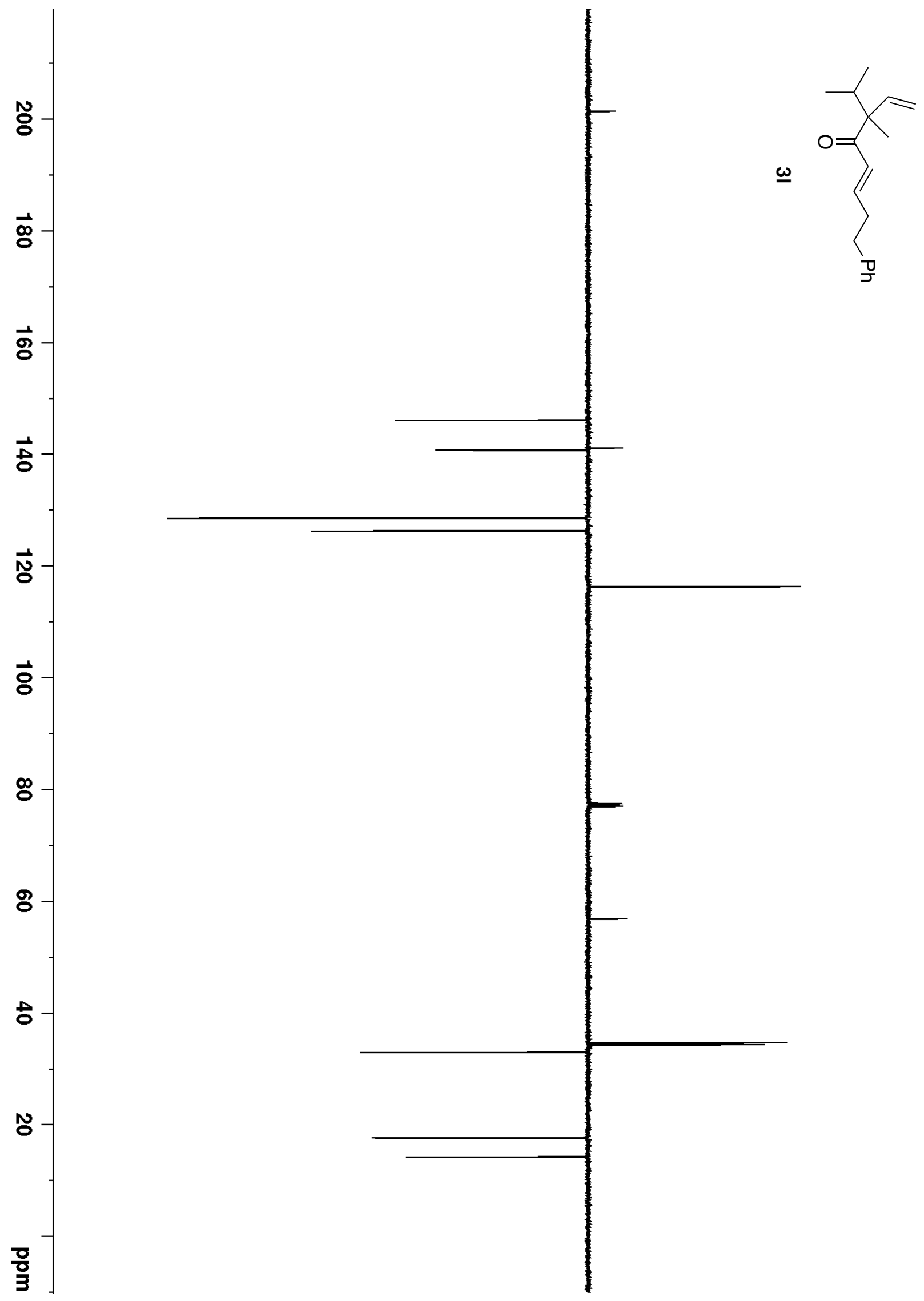

S92 


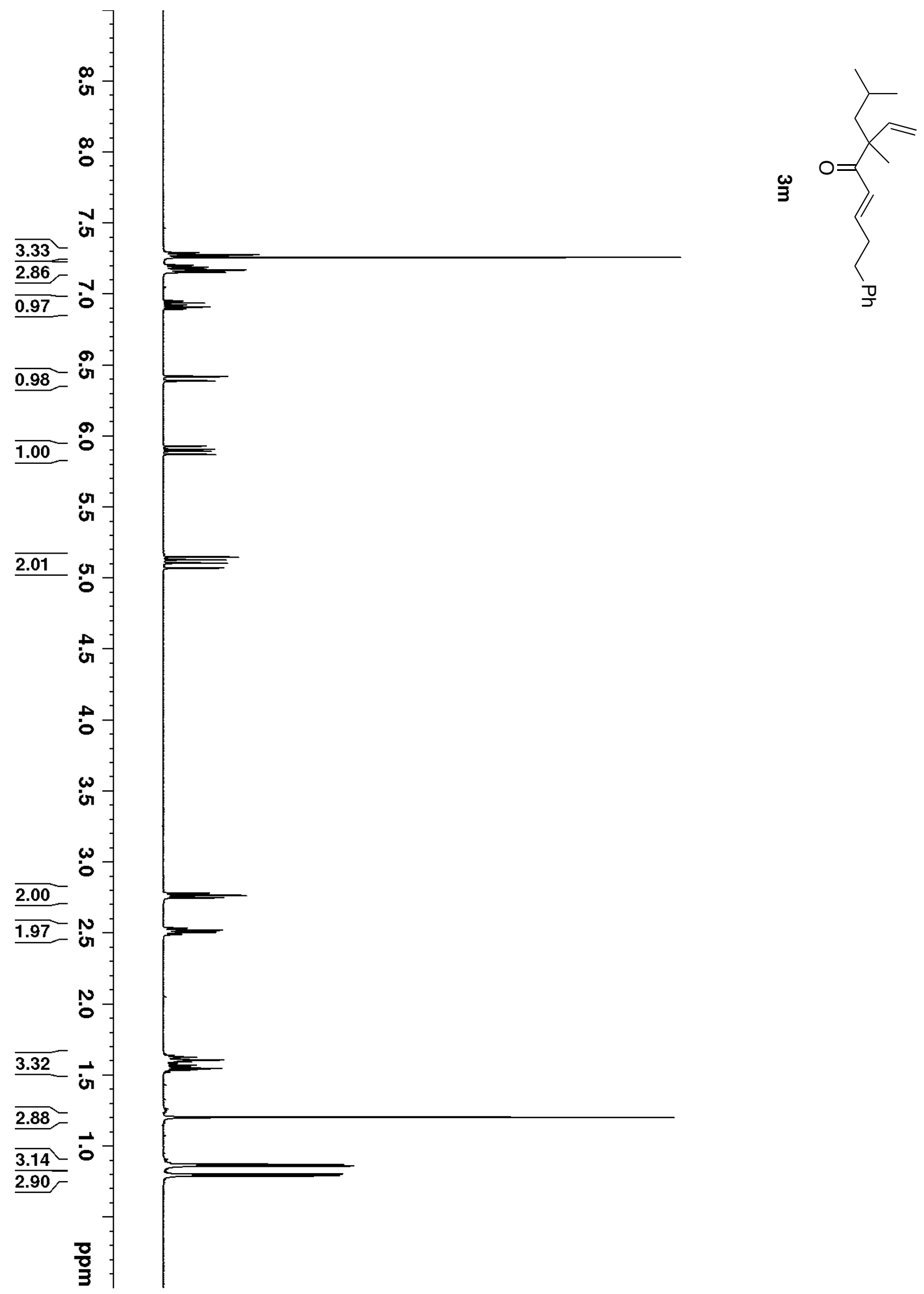




$$
1
$$




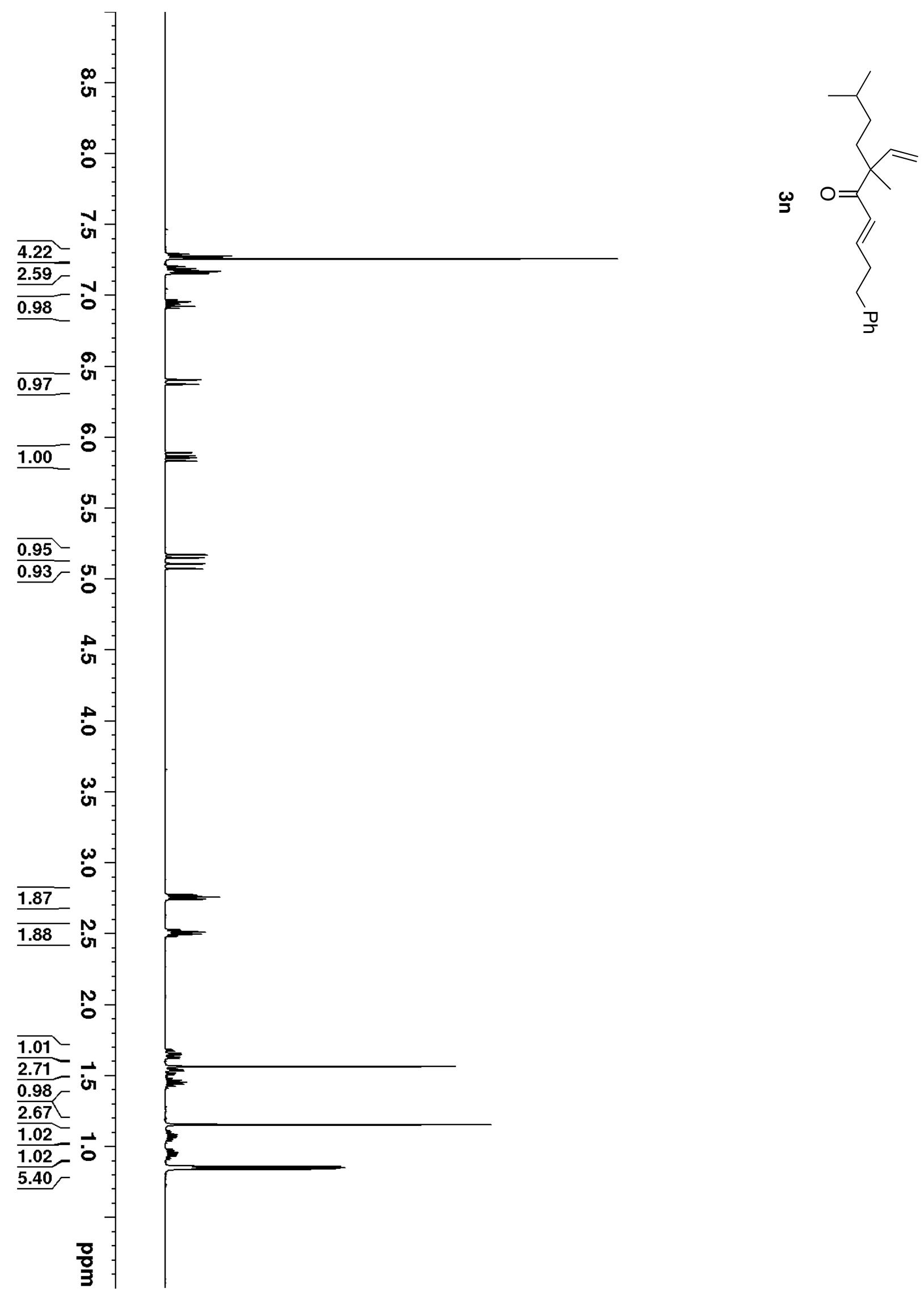




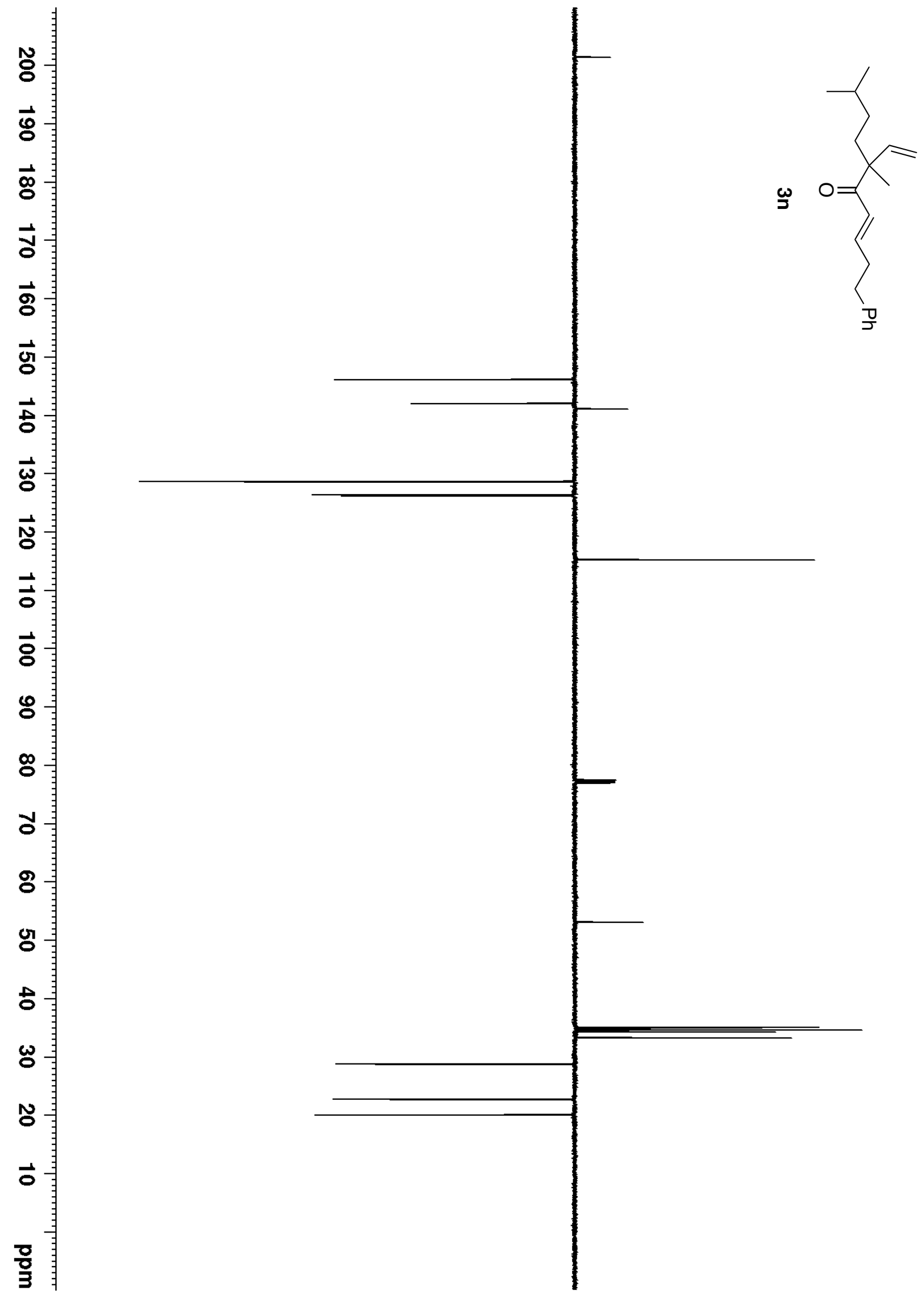




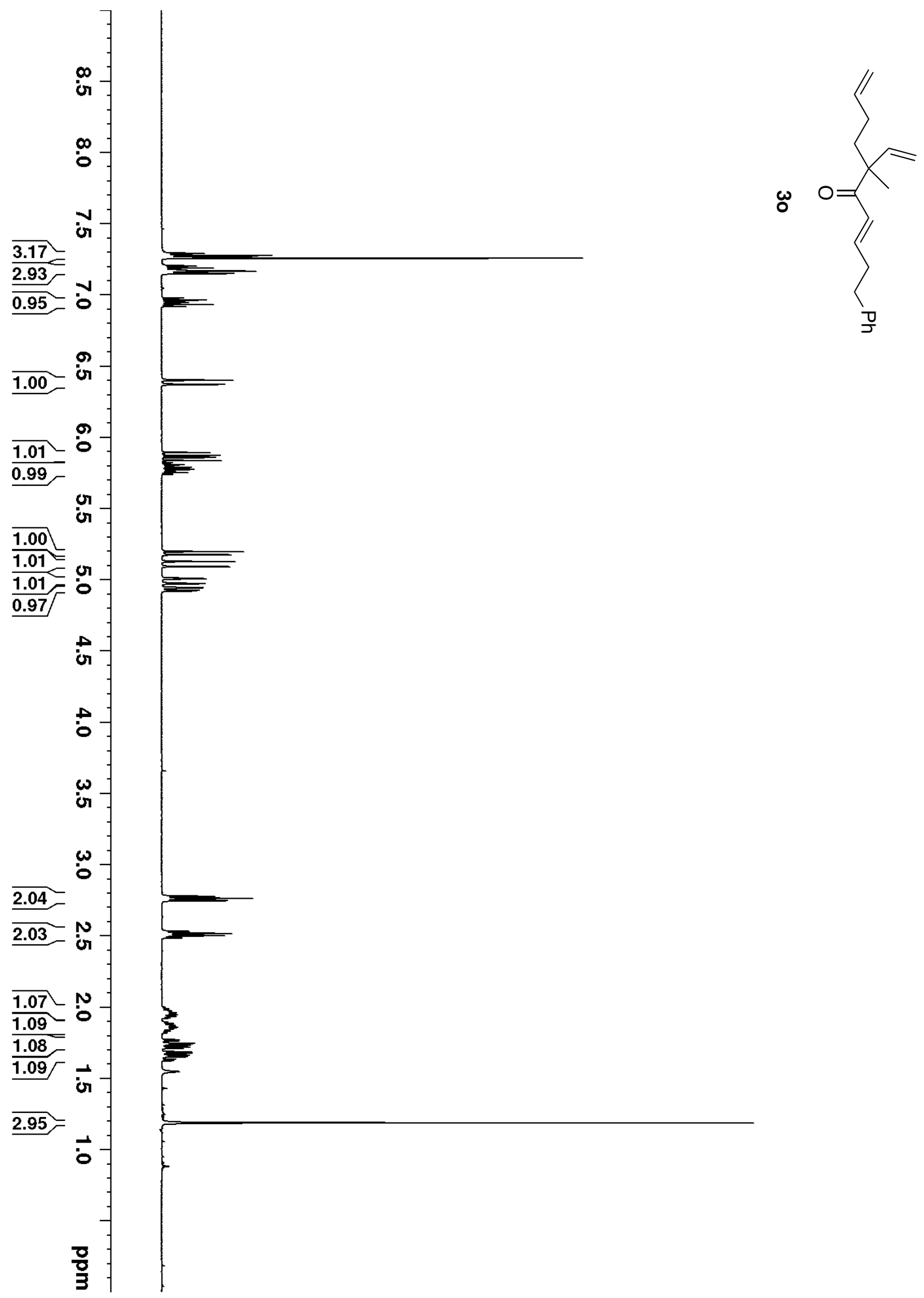




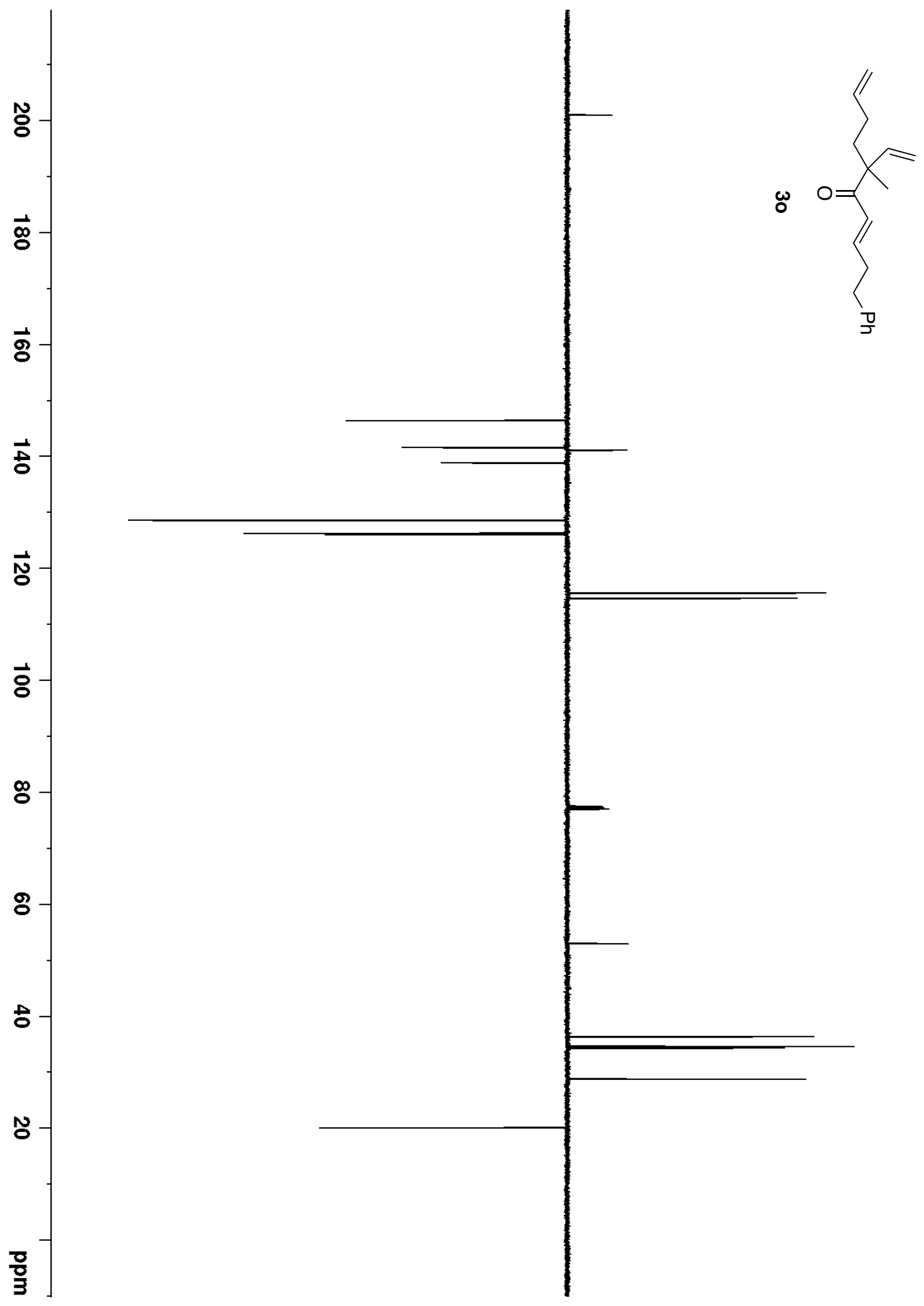




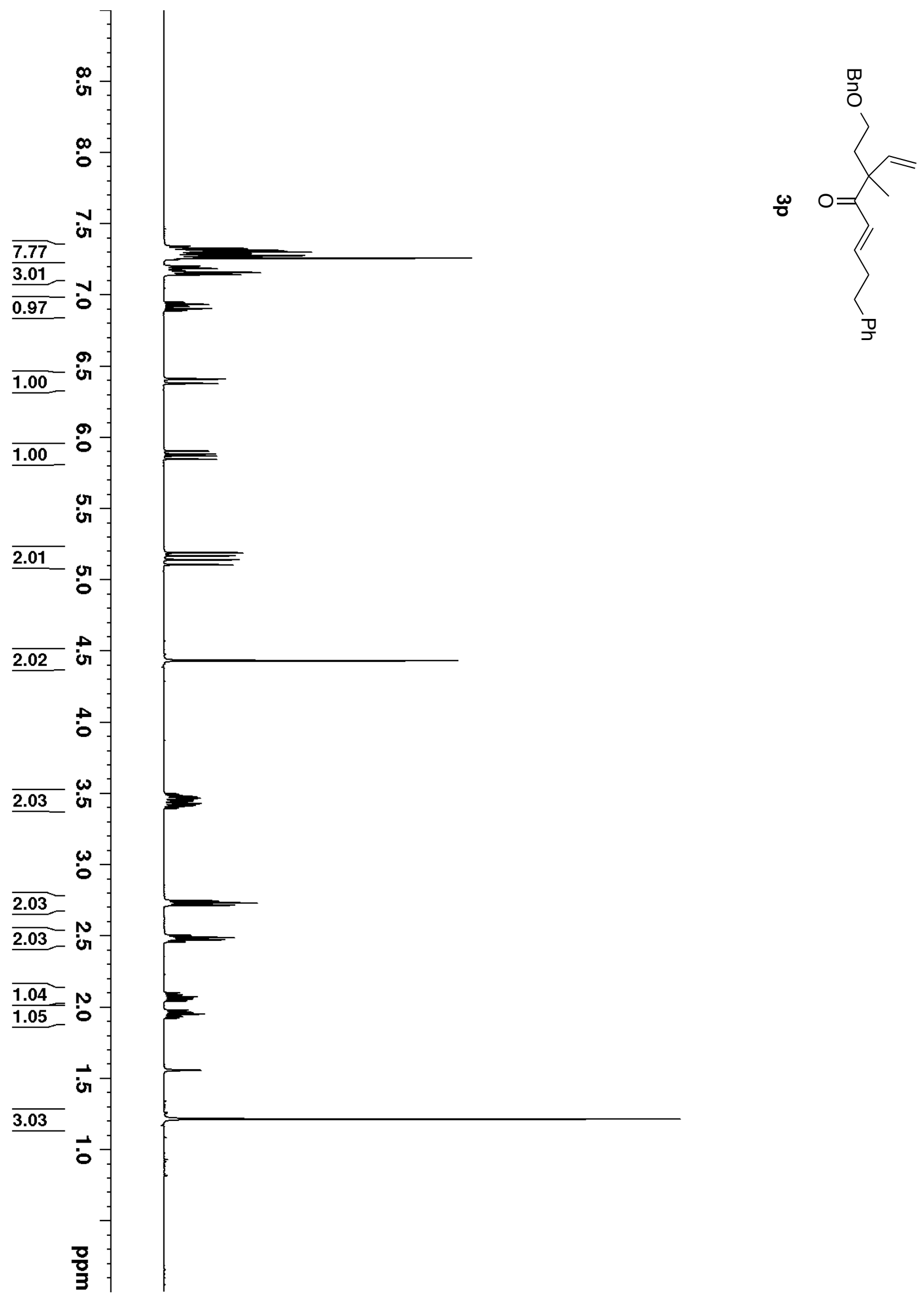




$$
f^{+1}
$$




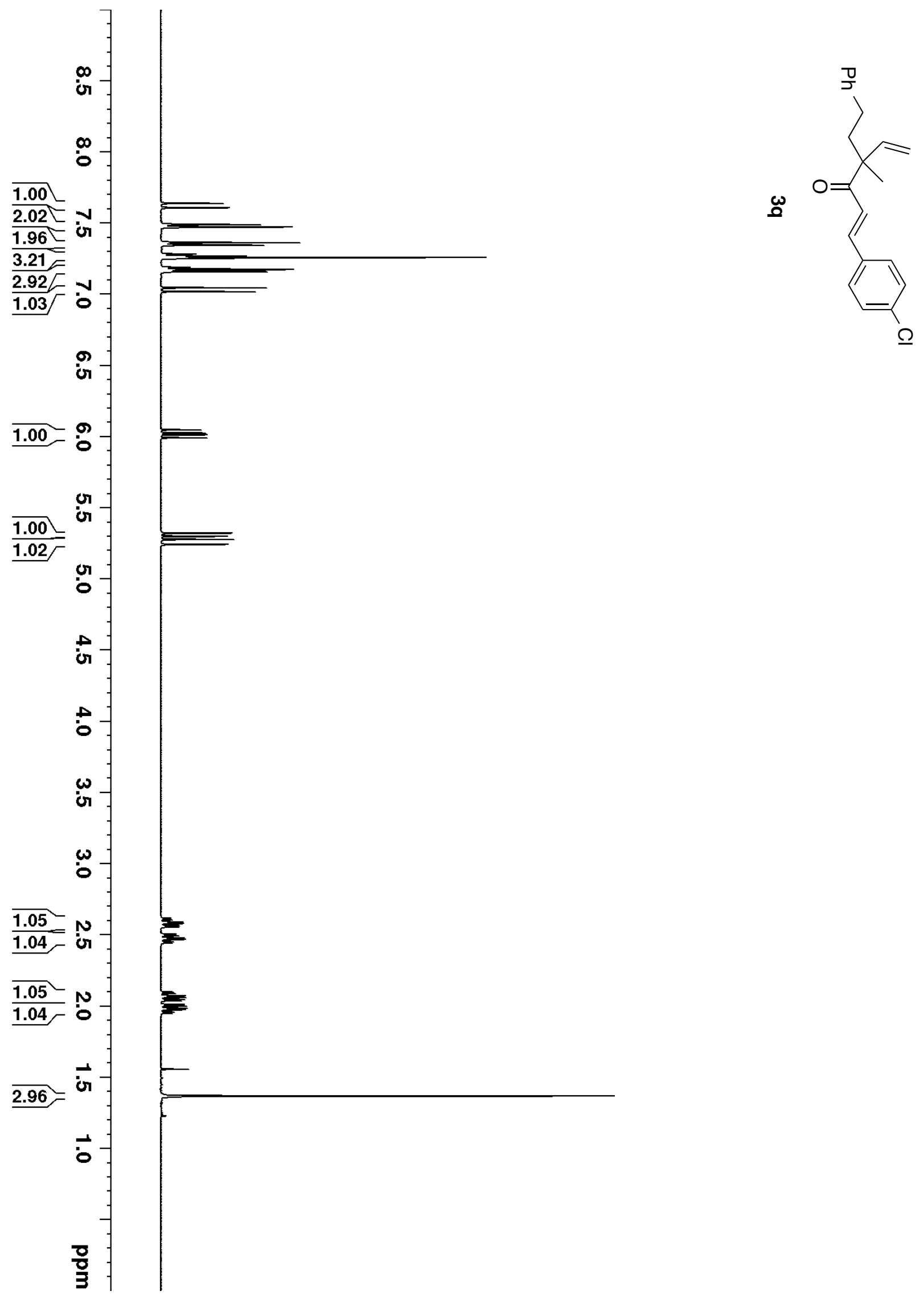




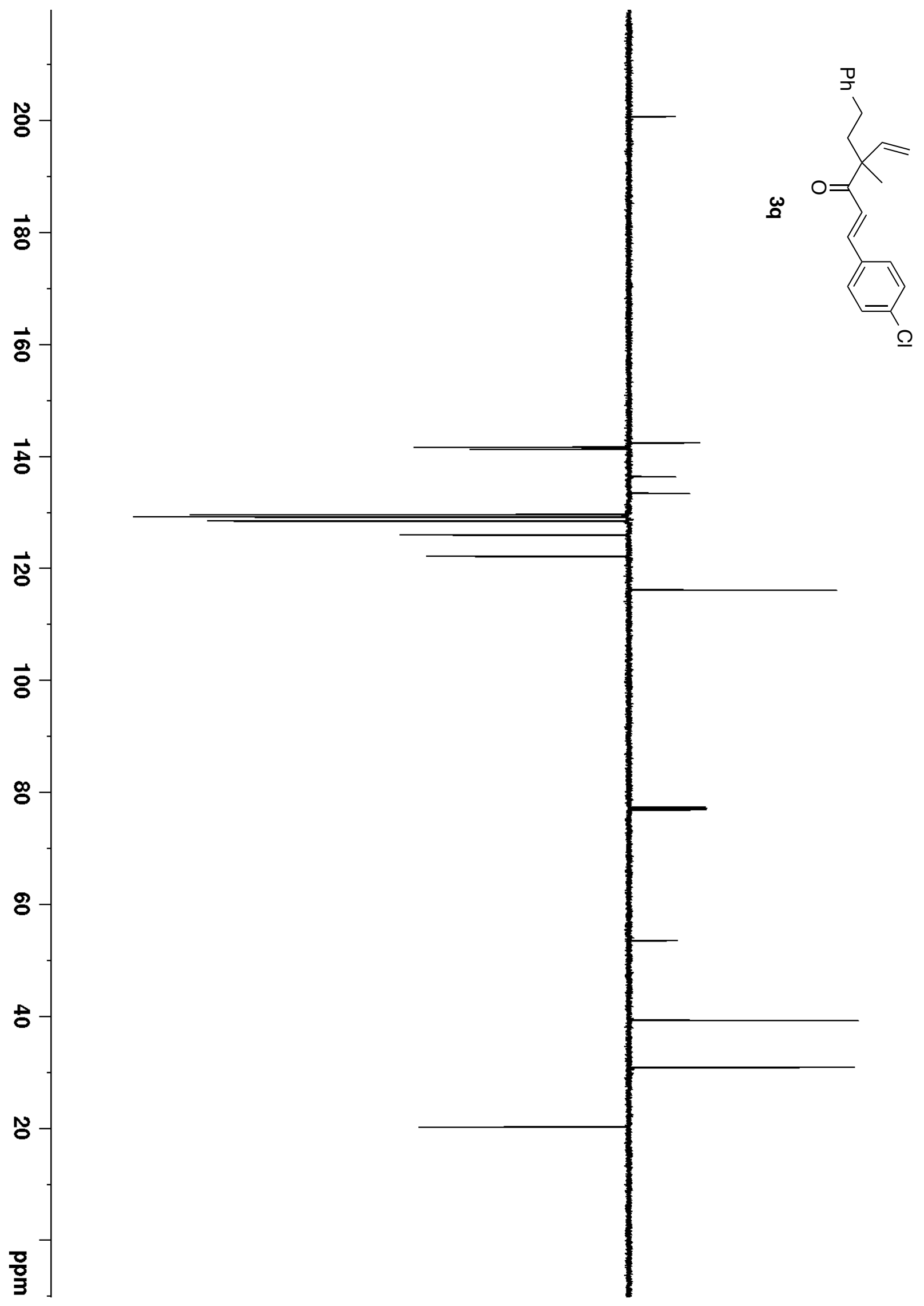



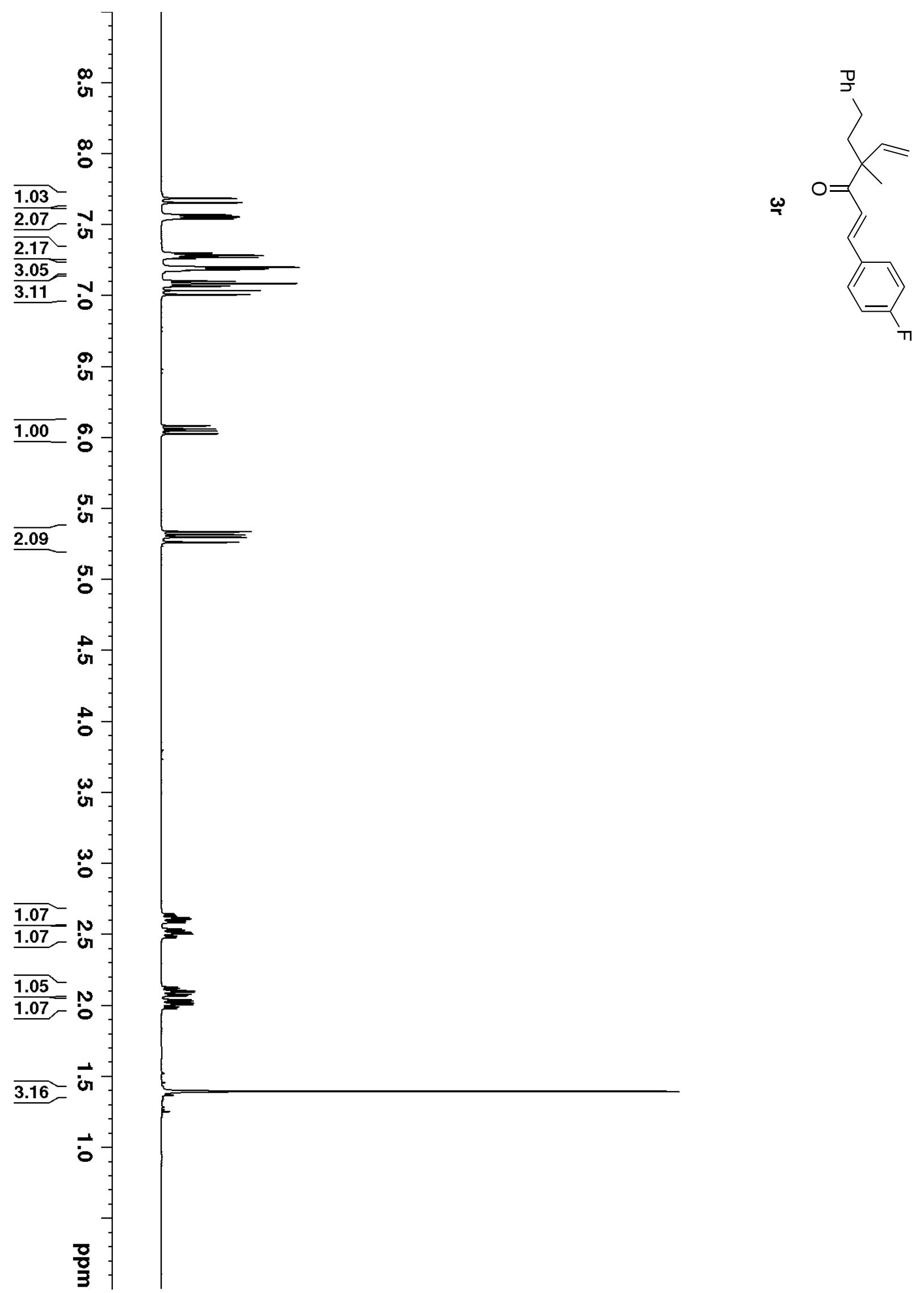


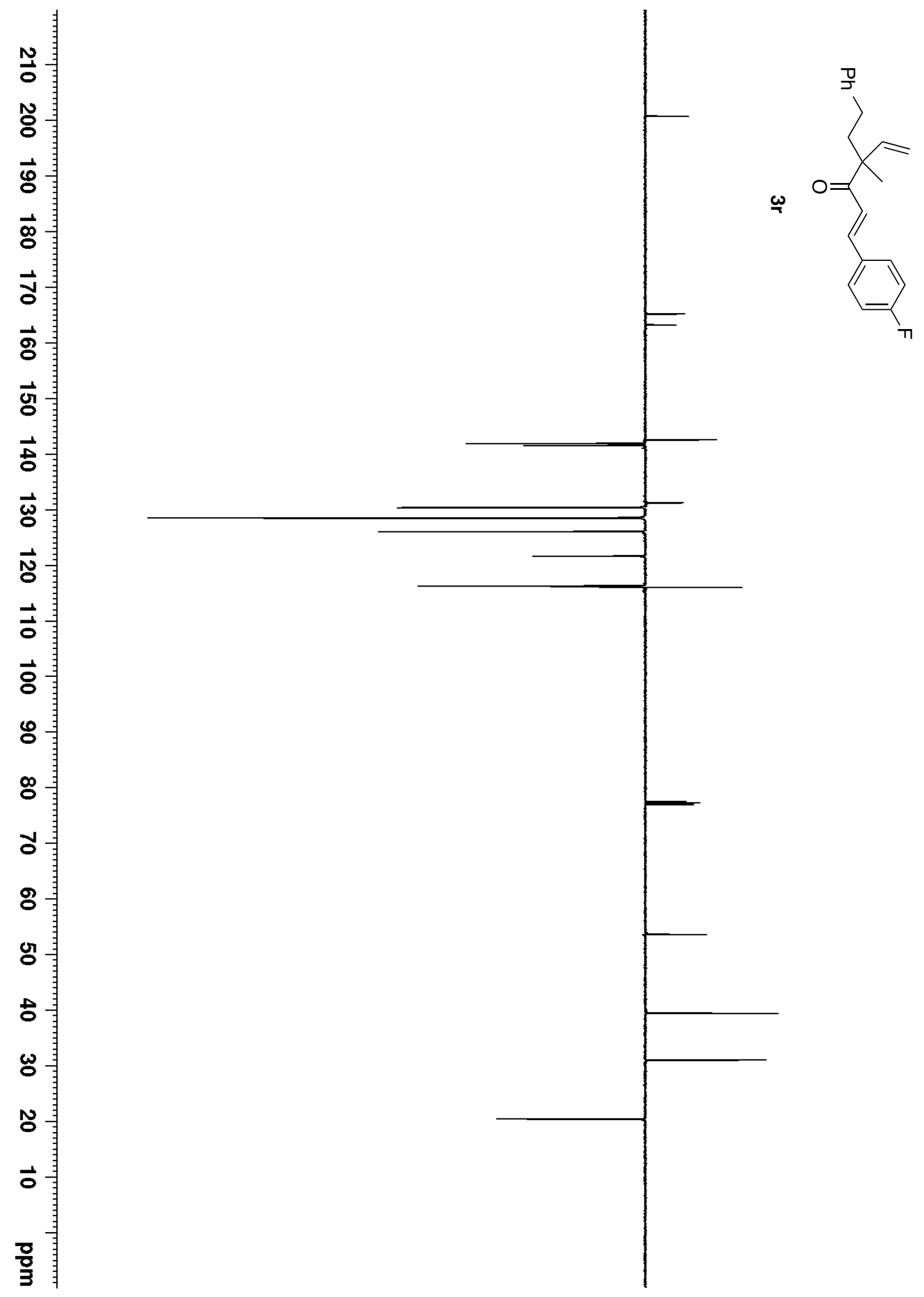




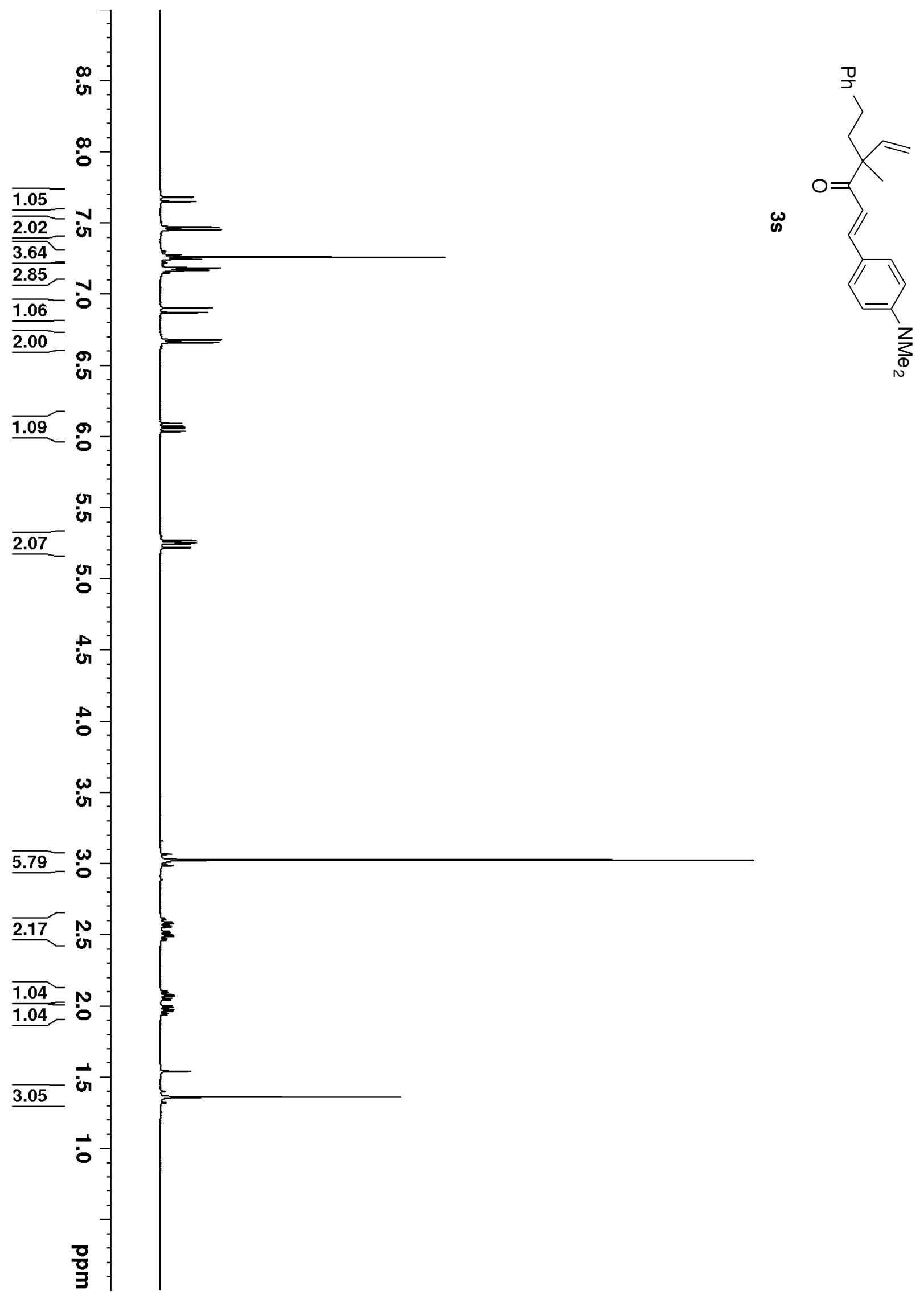




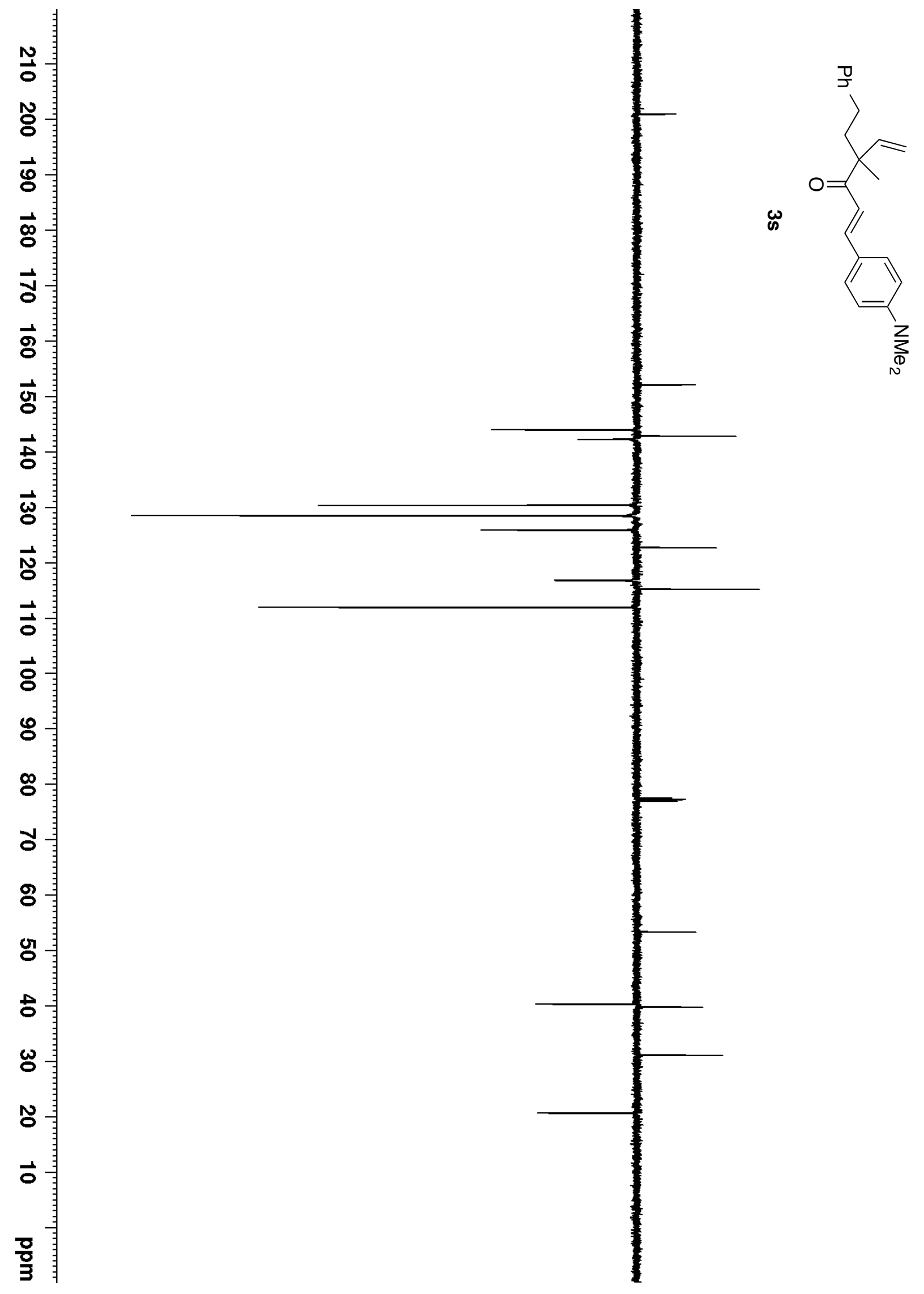



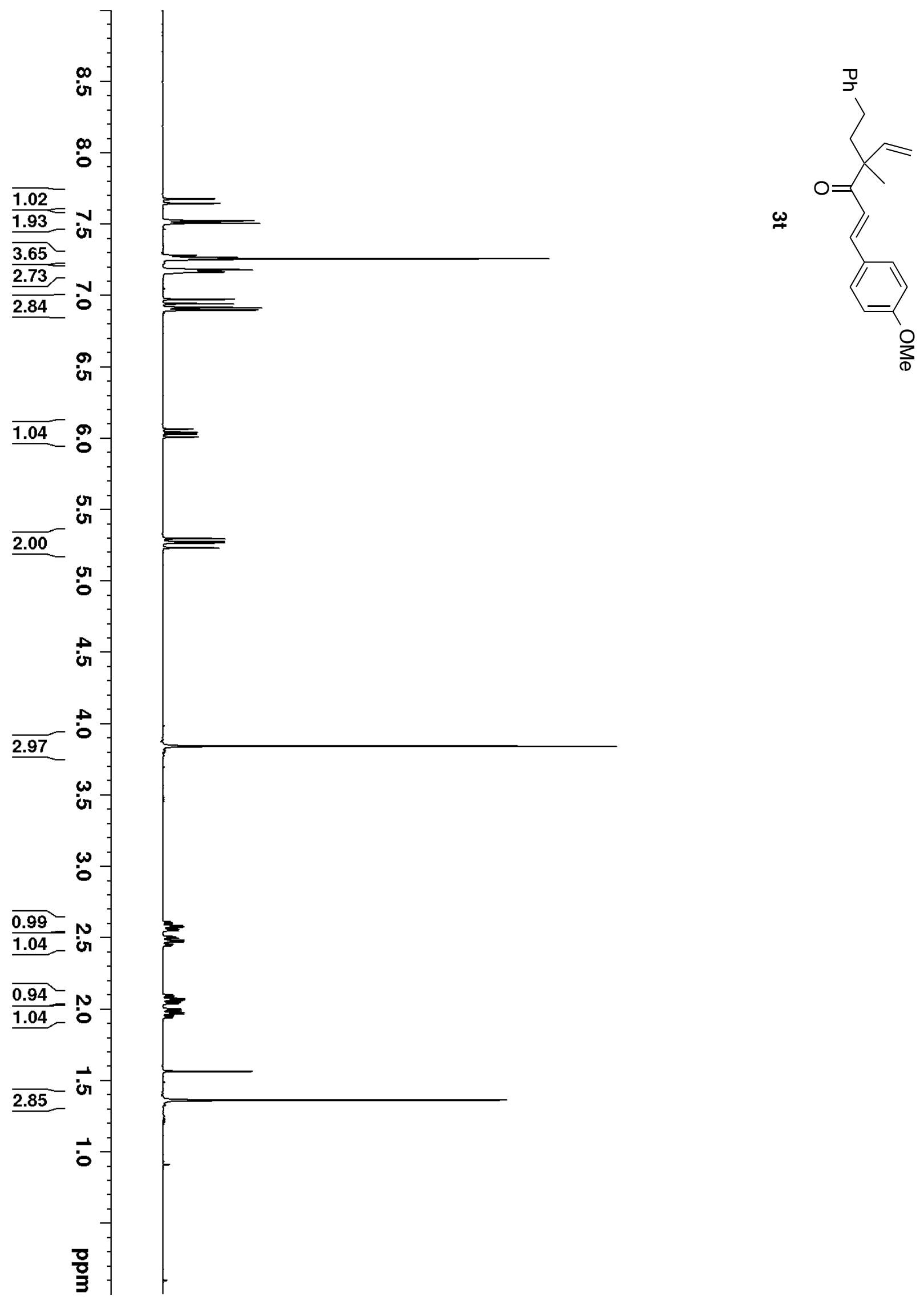


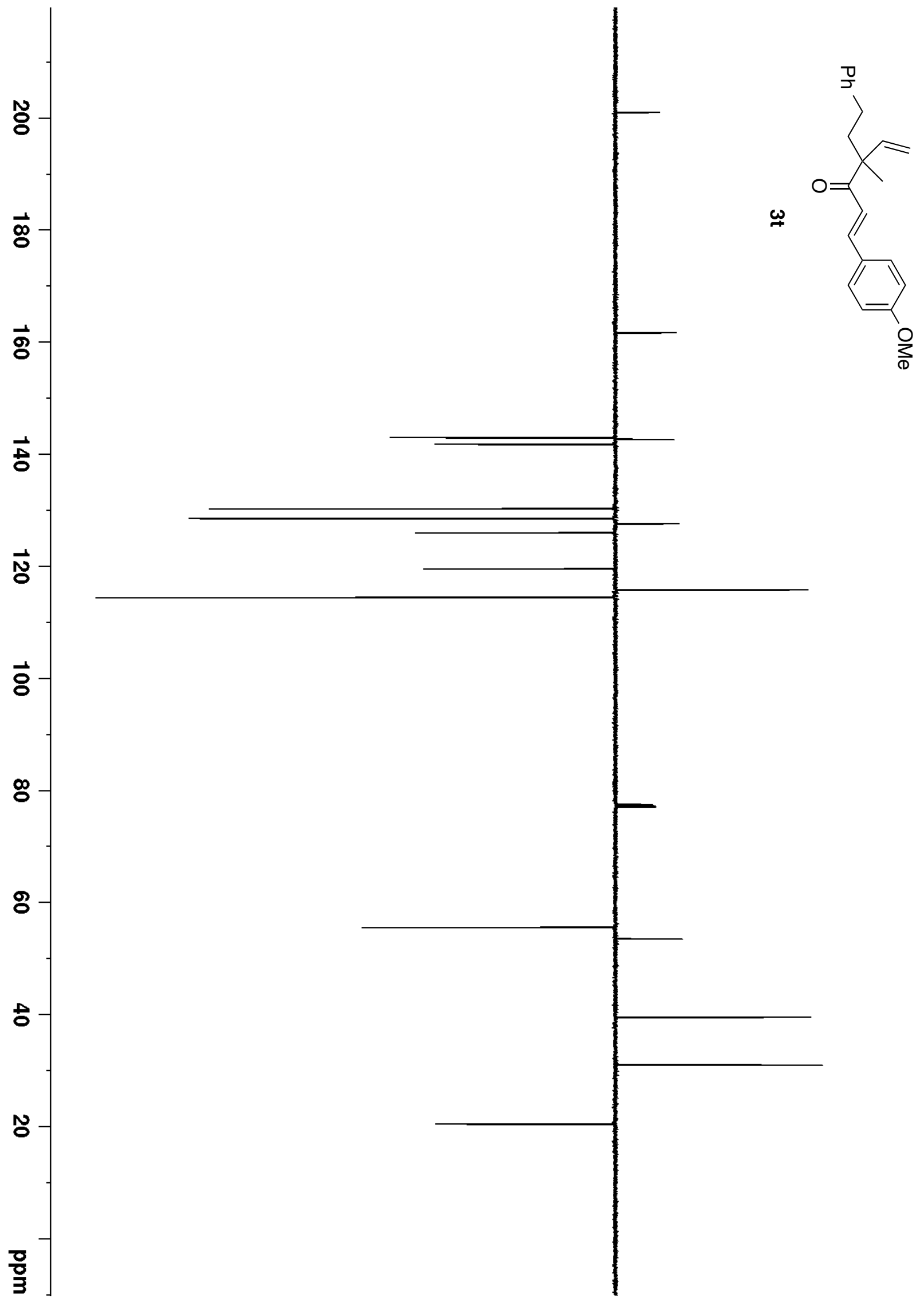



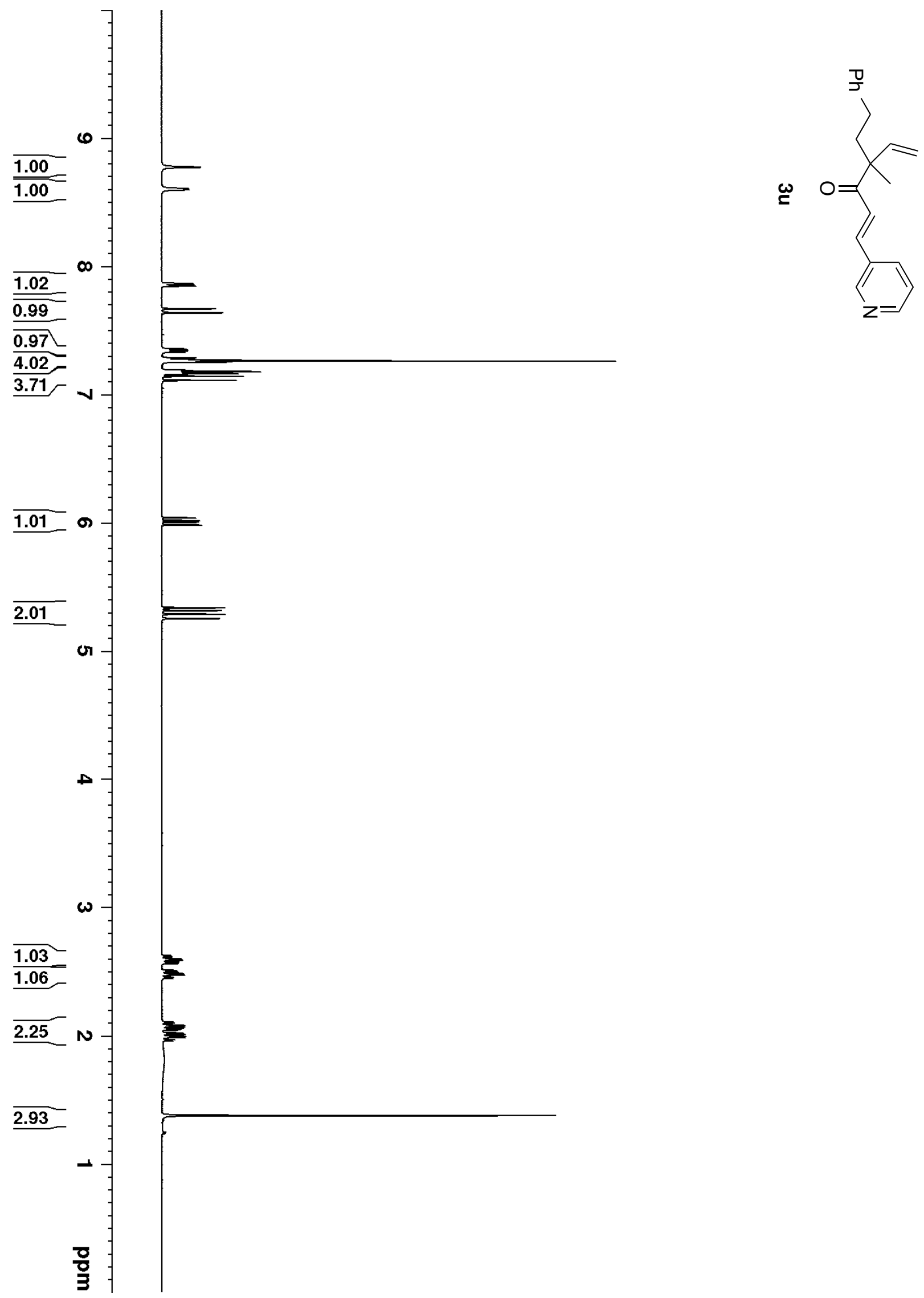


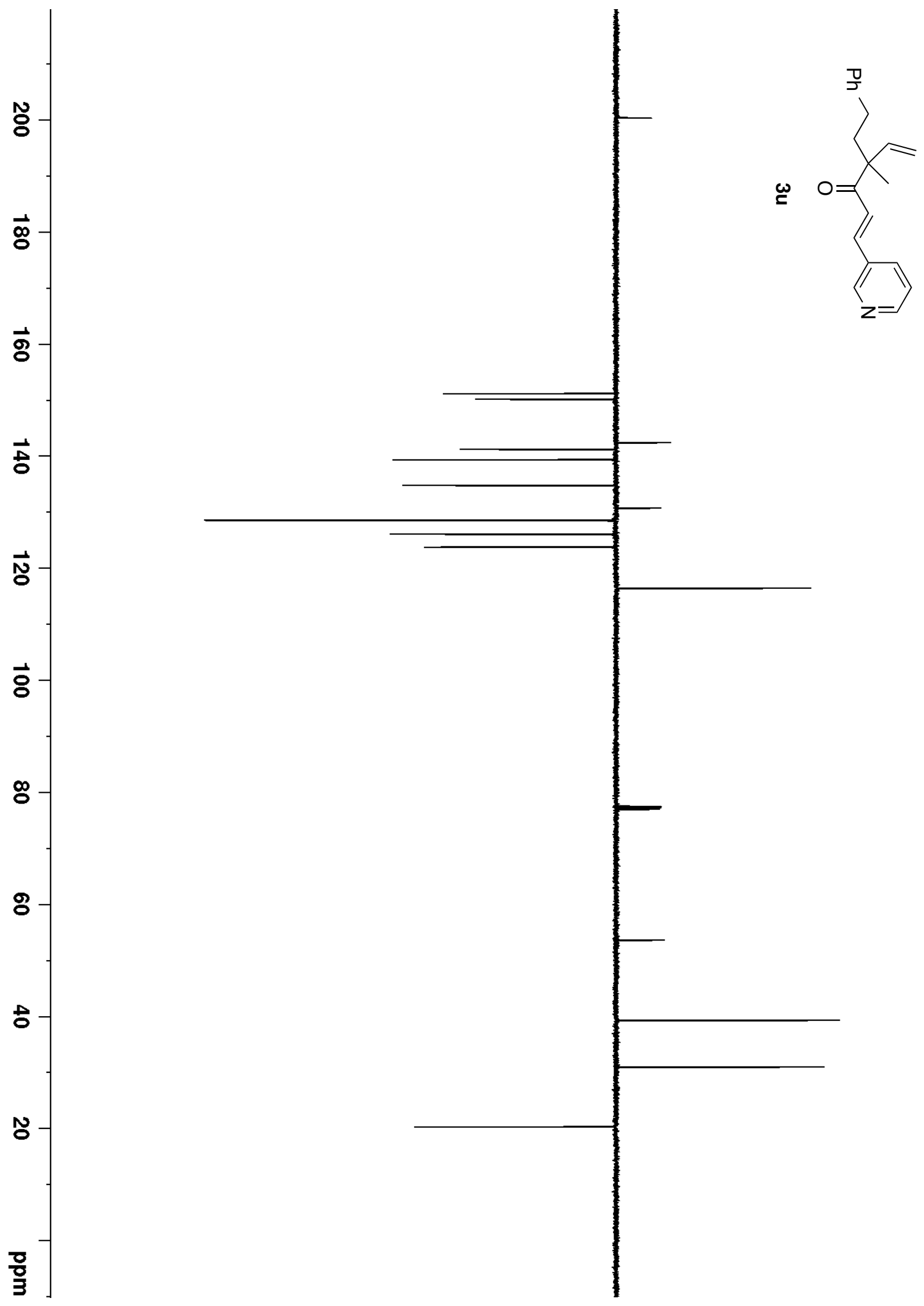




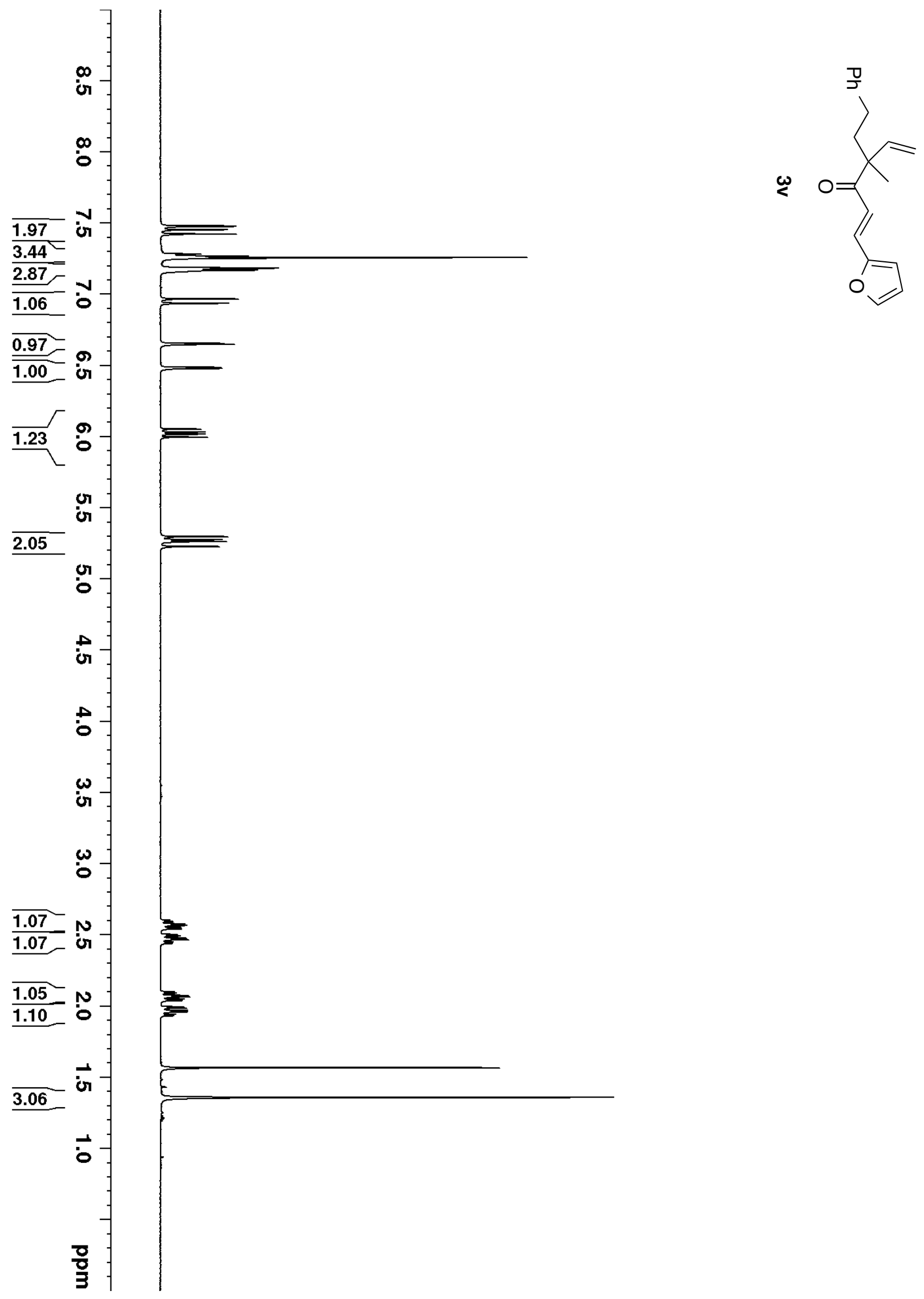




$$
1=
$$




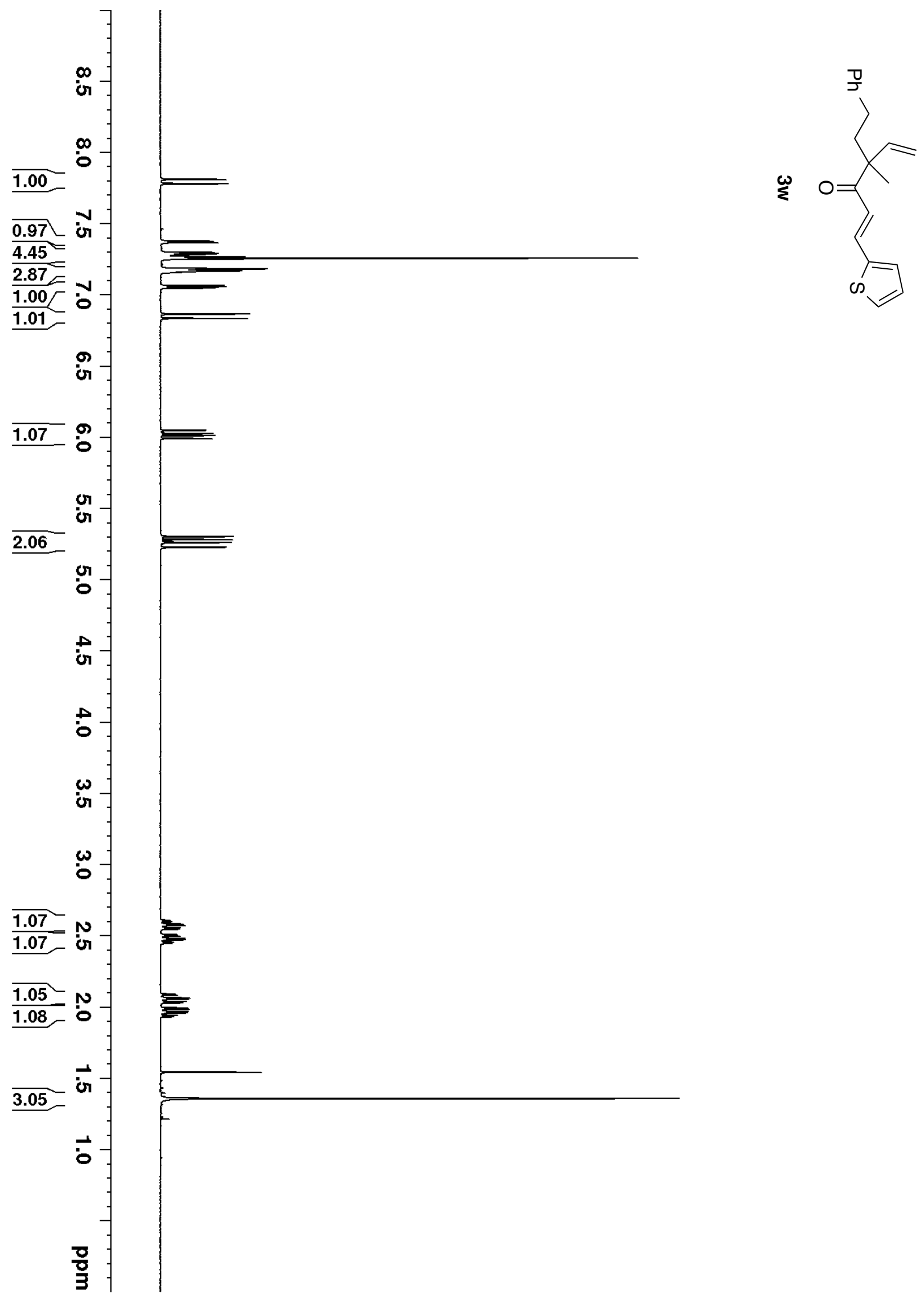




$$
1+
$$



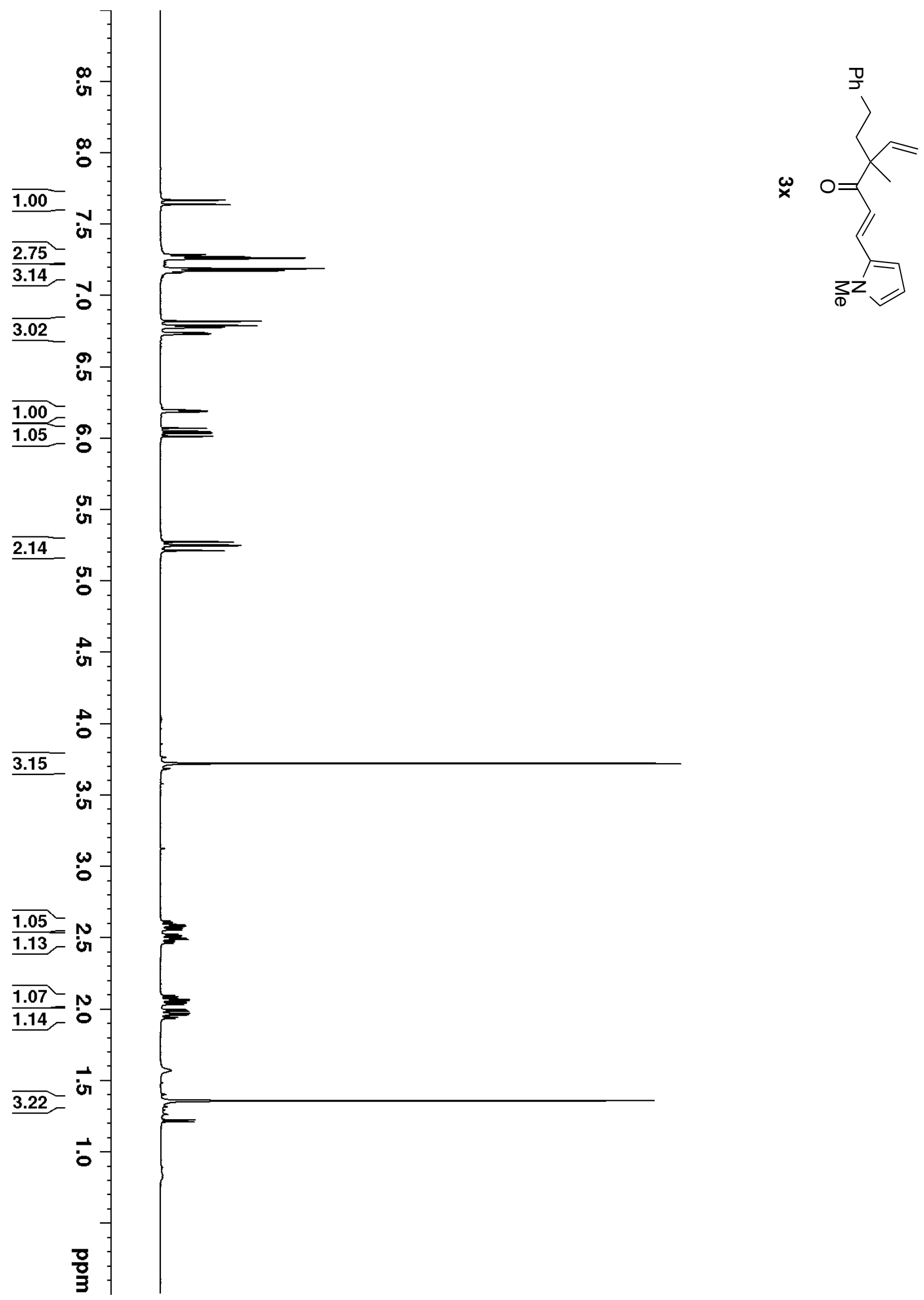


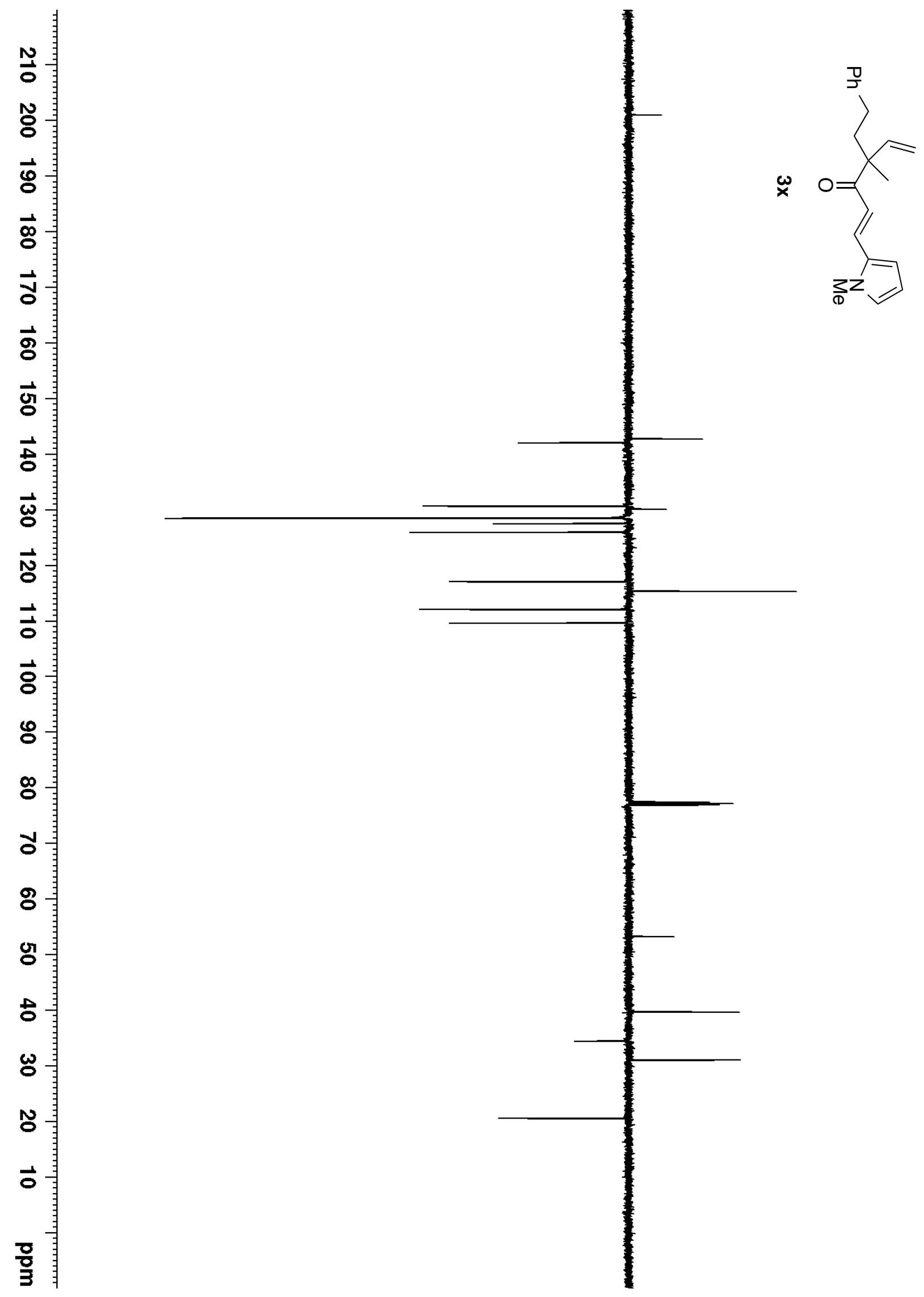



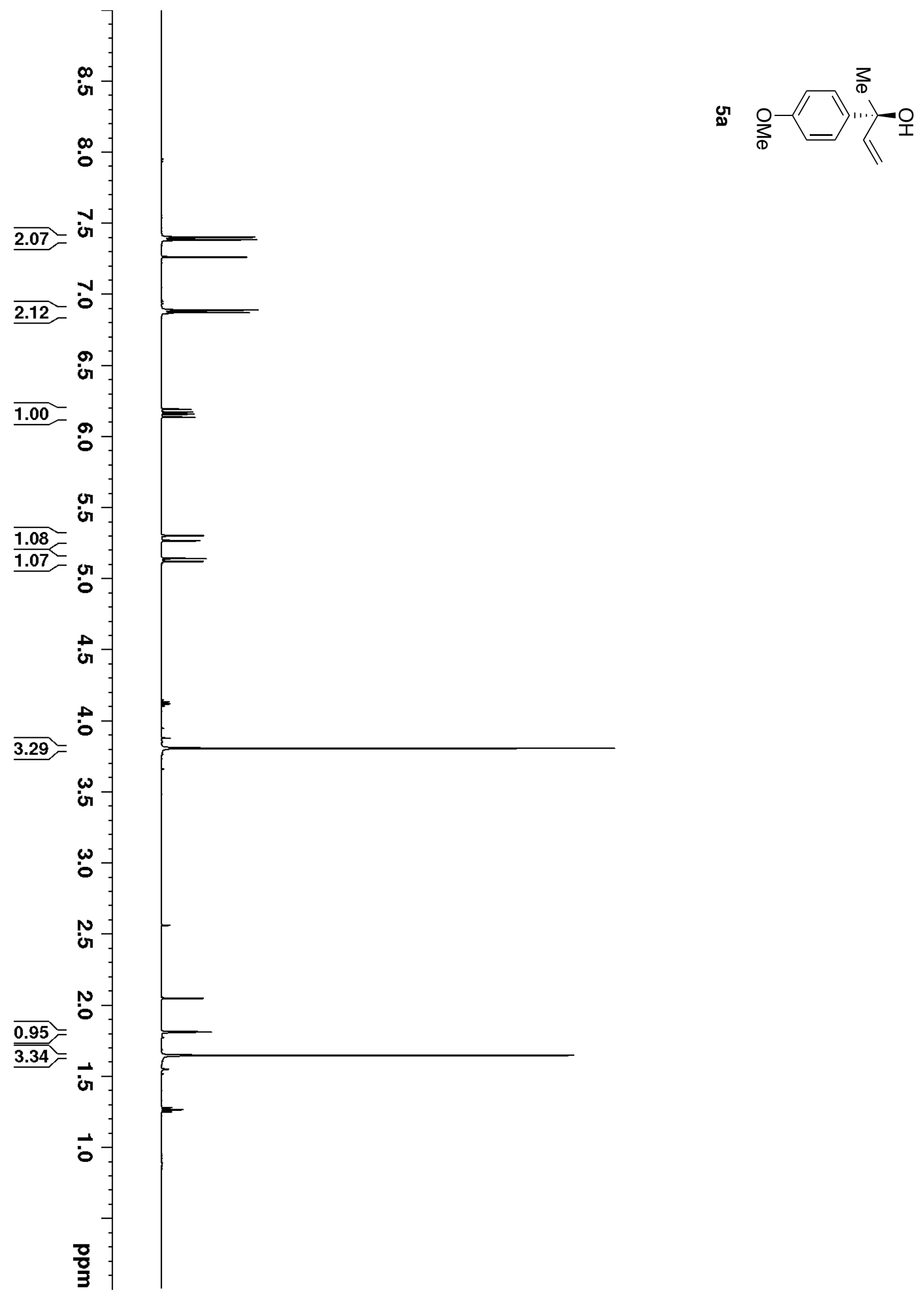


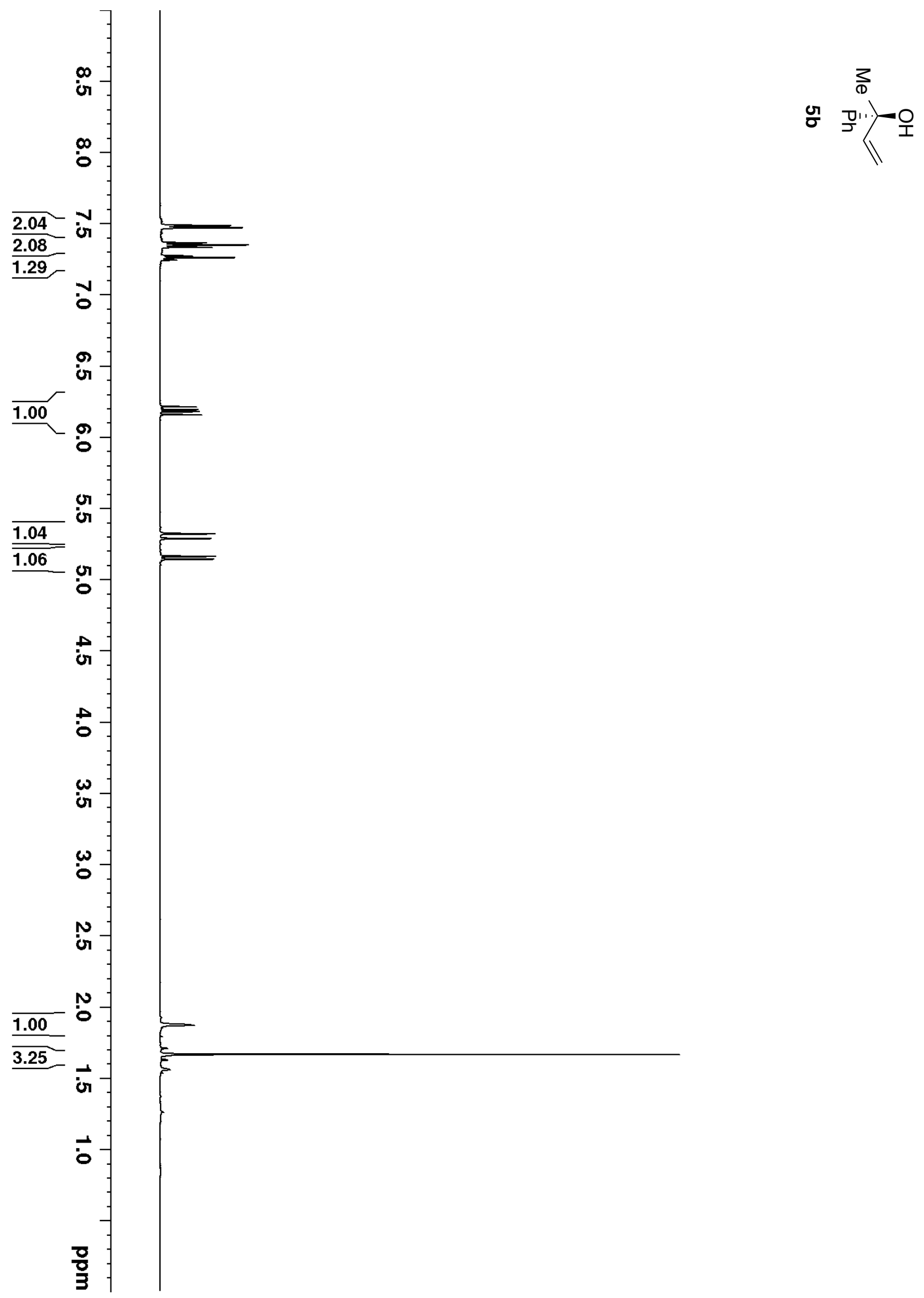



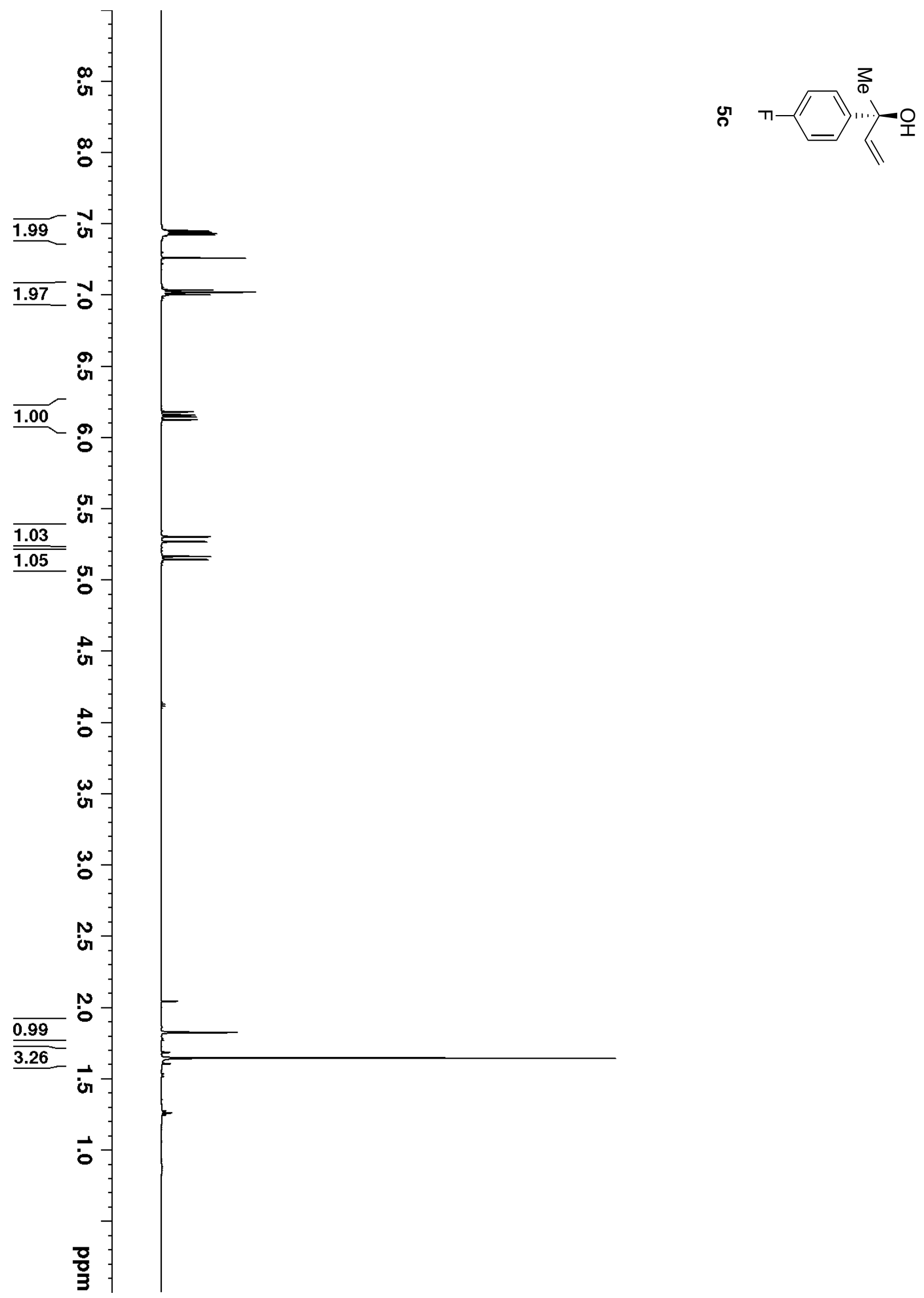

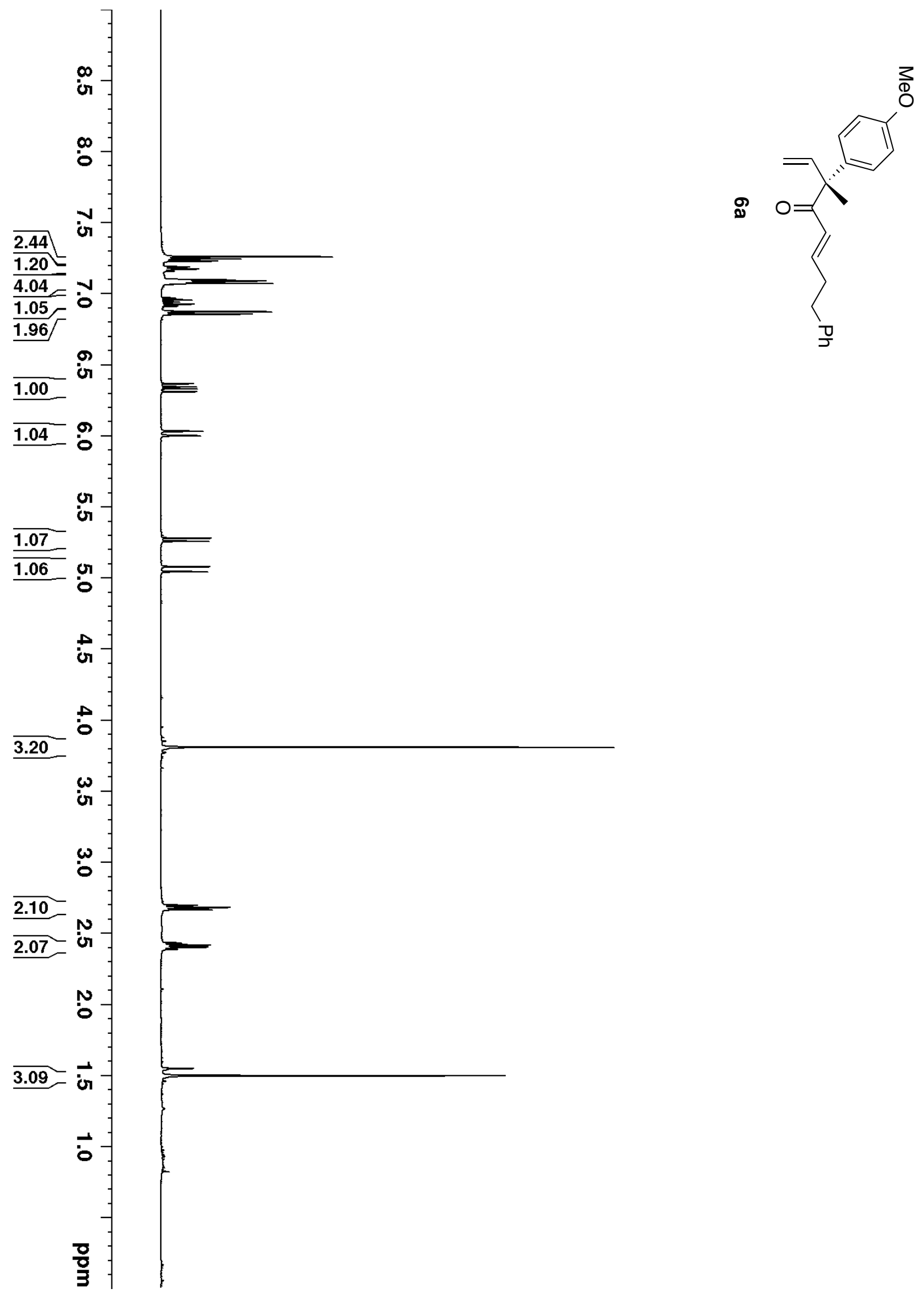


$$
\mid=
$$




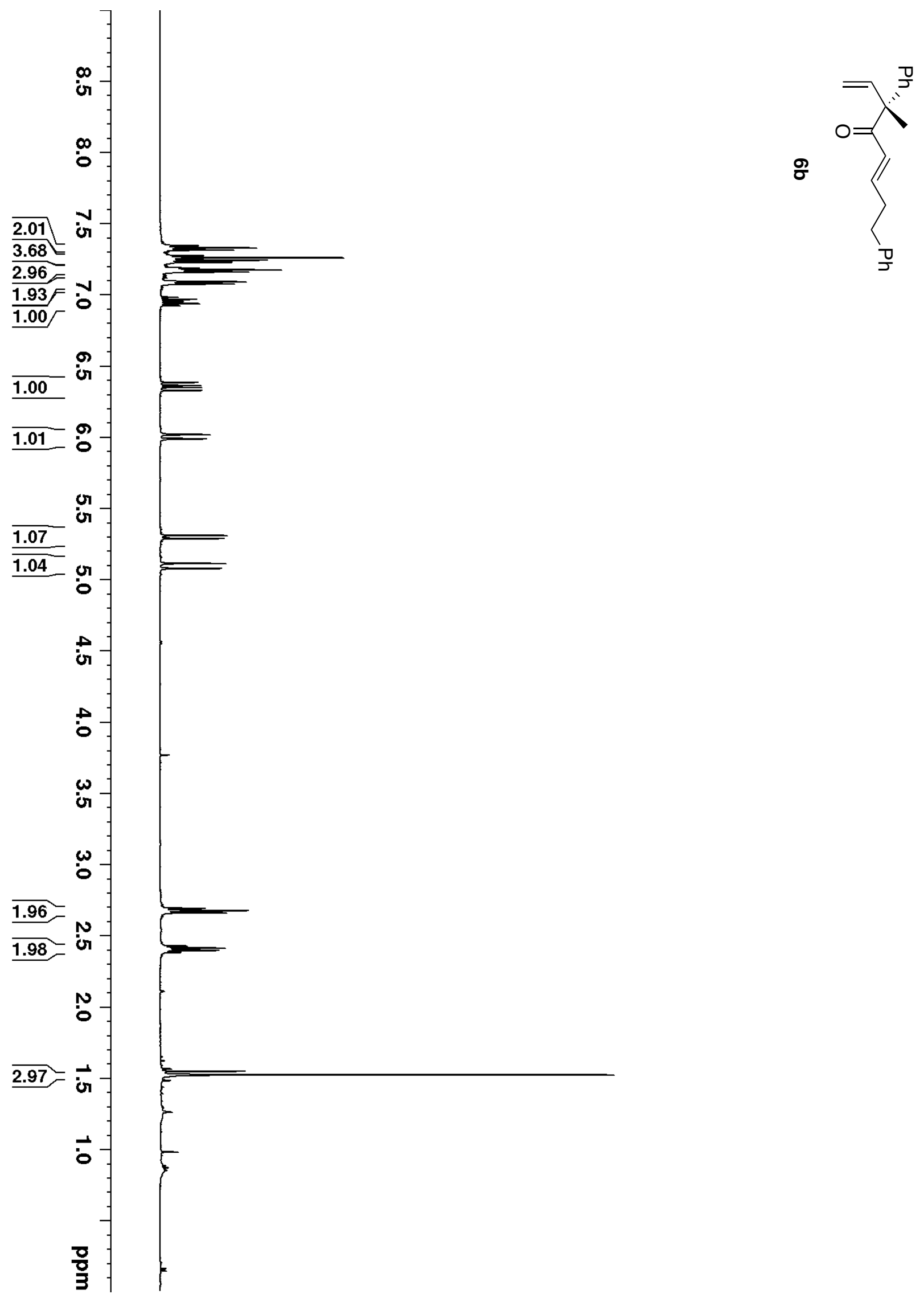




$$
H
$$




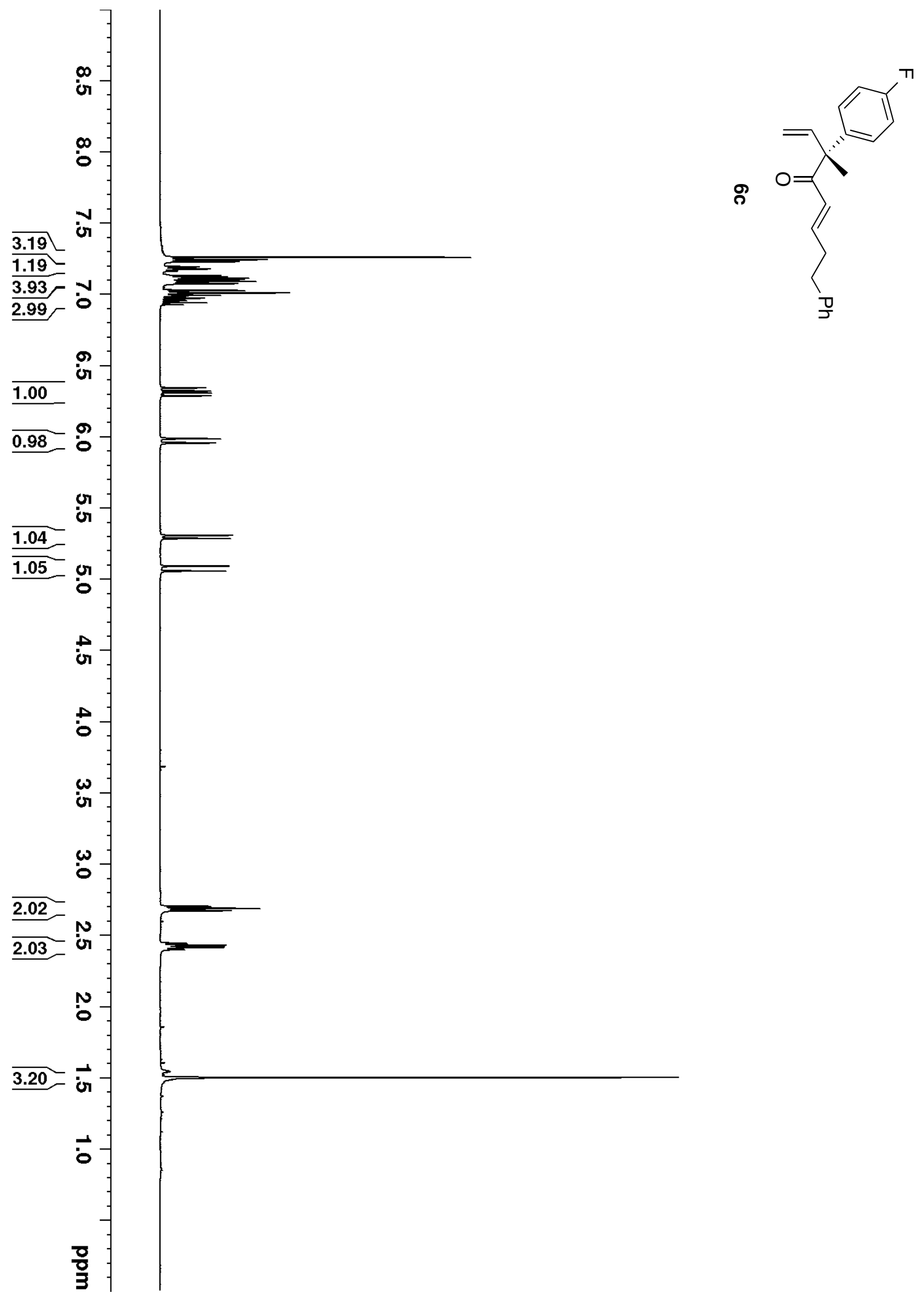




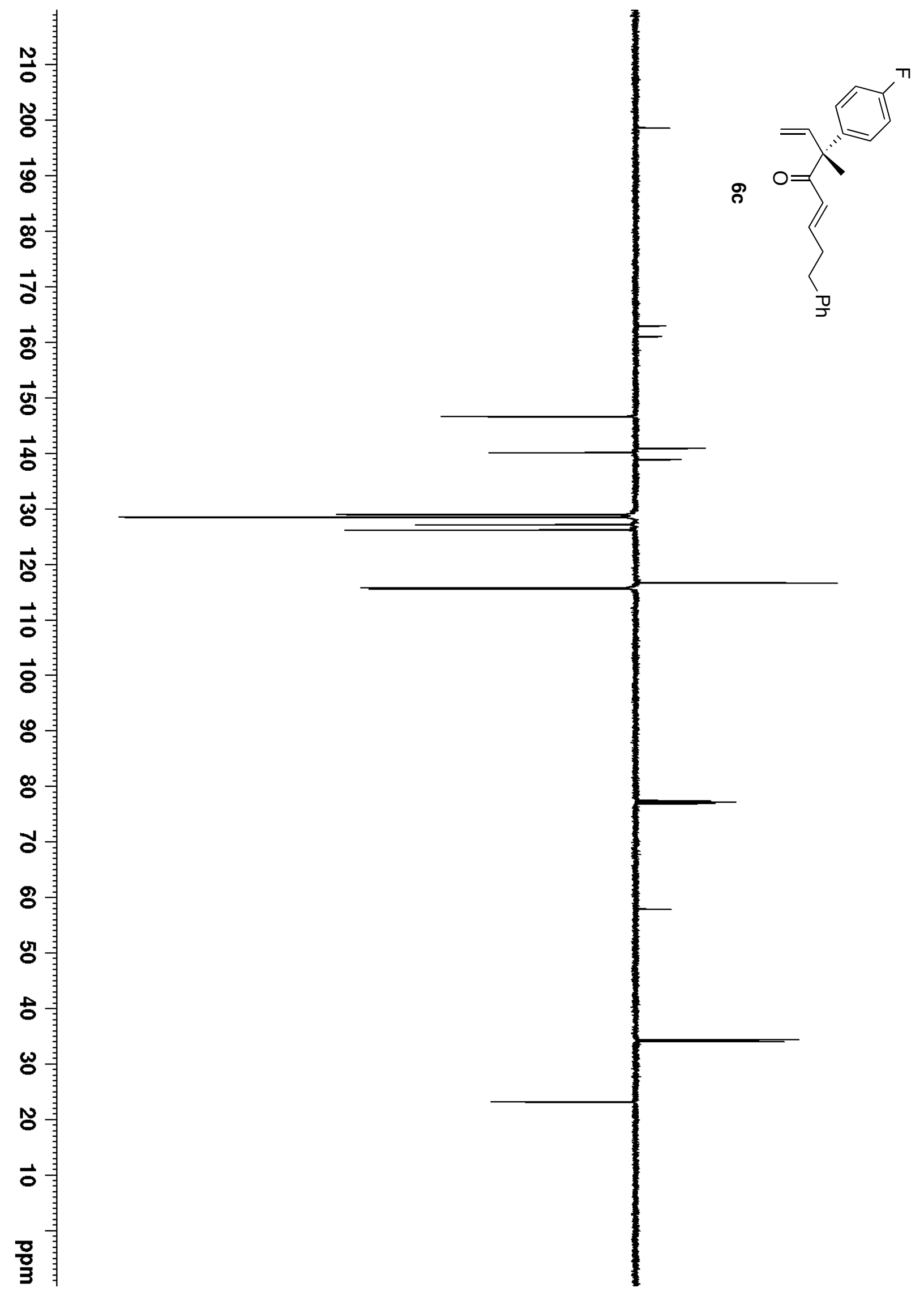




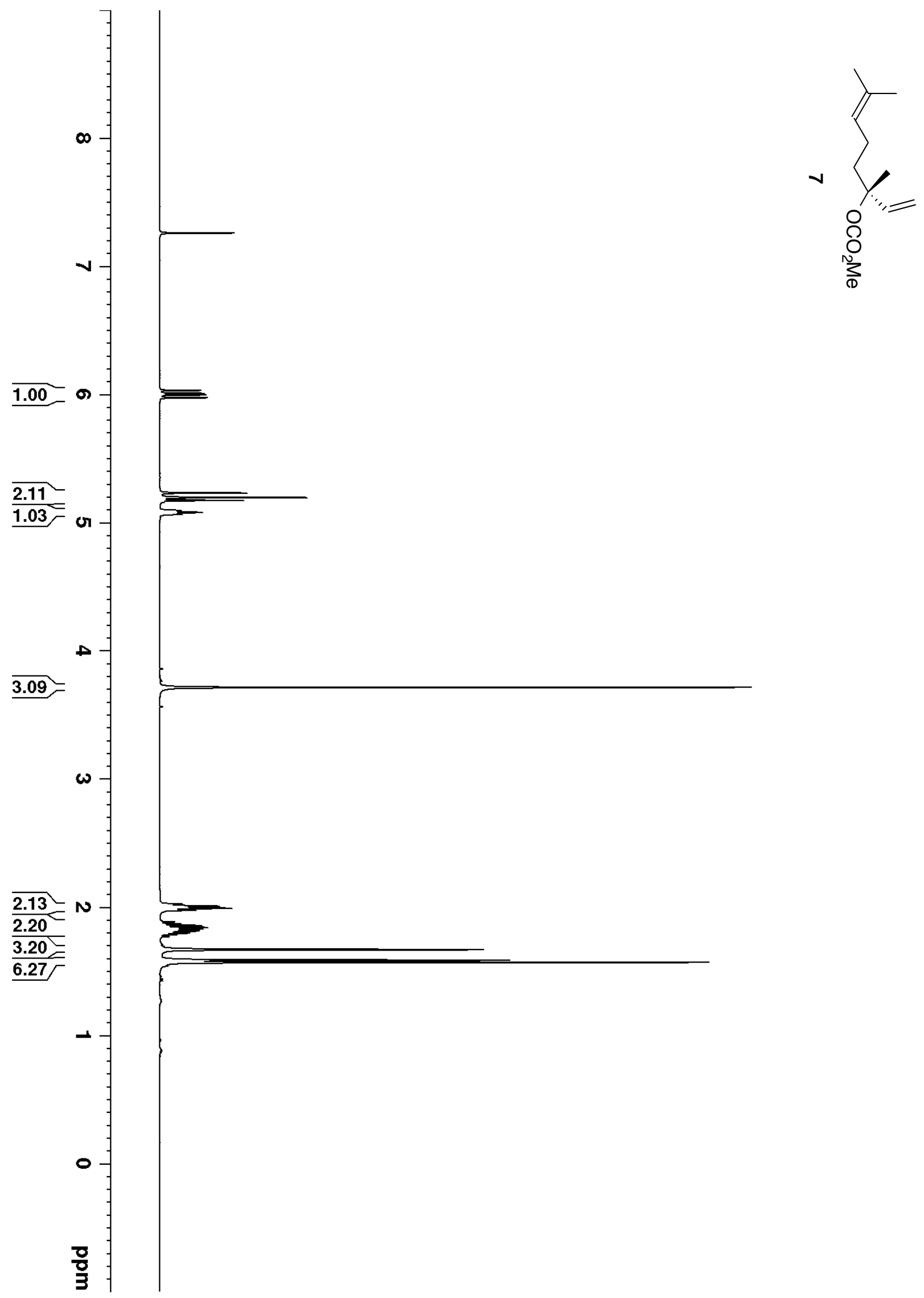



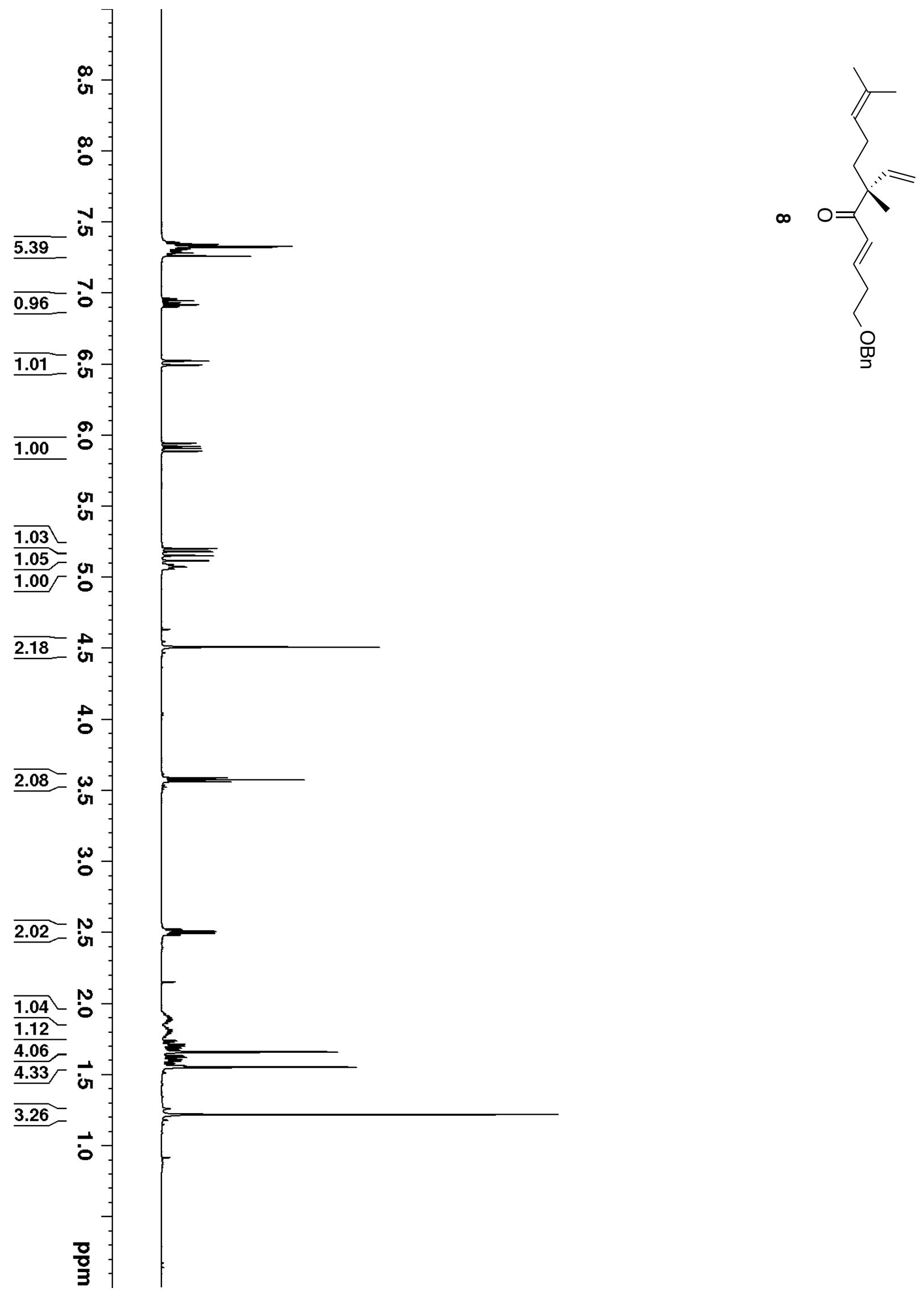


$$
\text { |ft }
$$




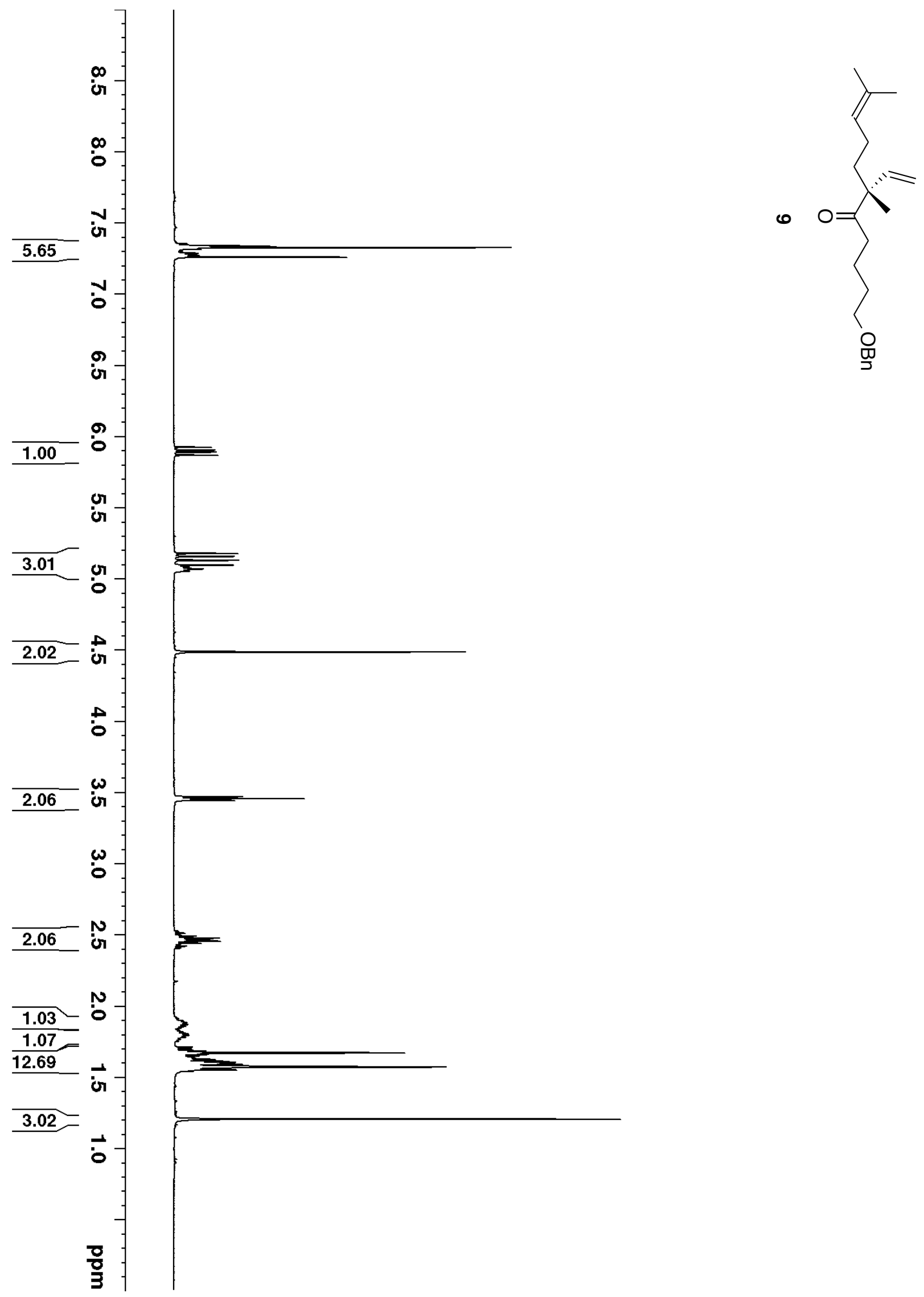




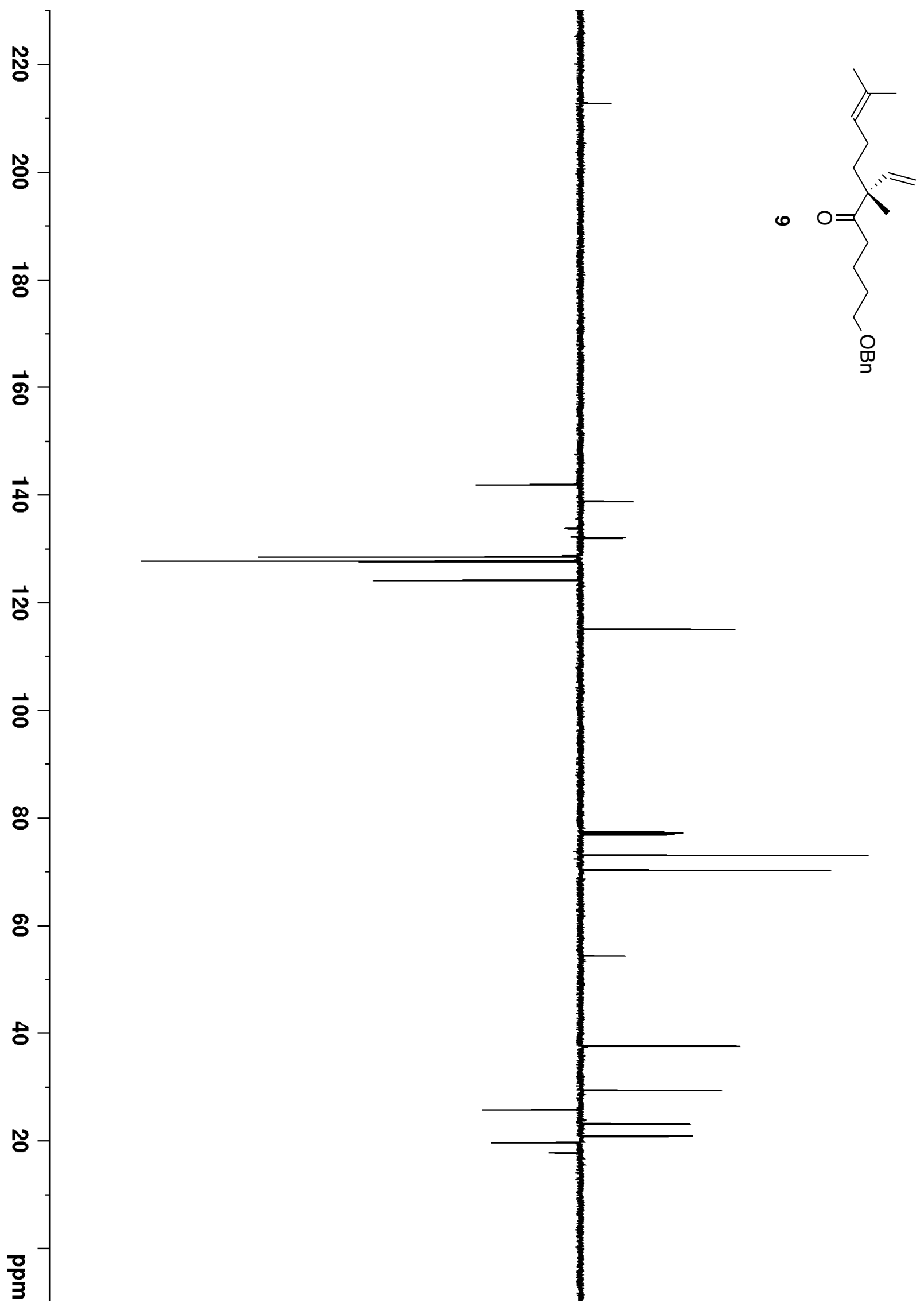




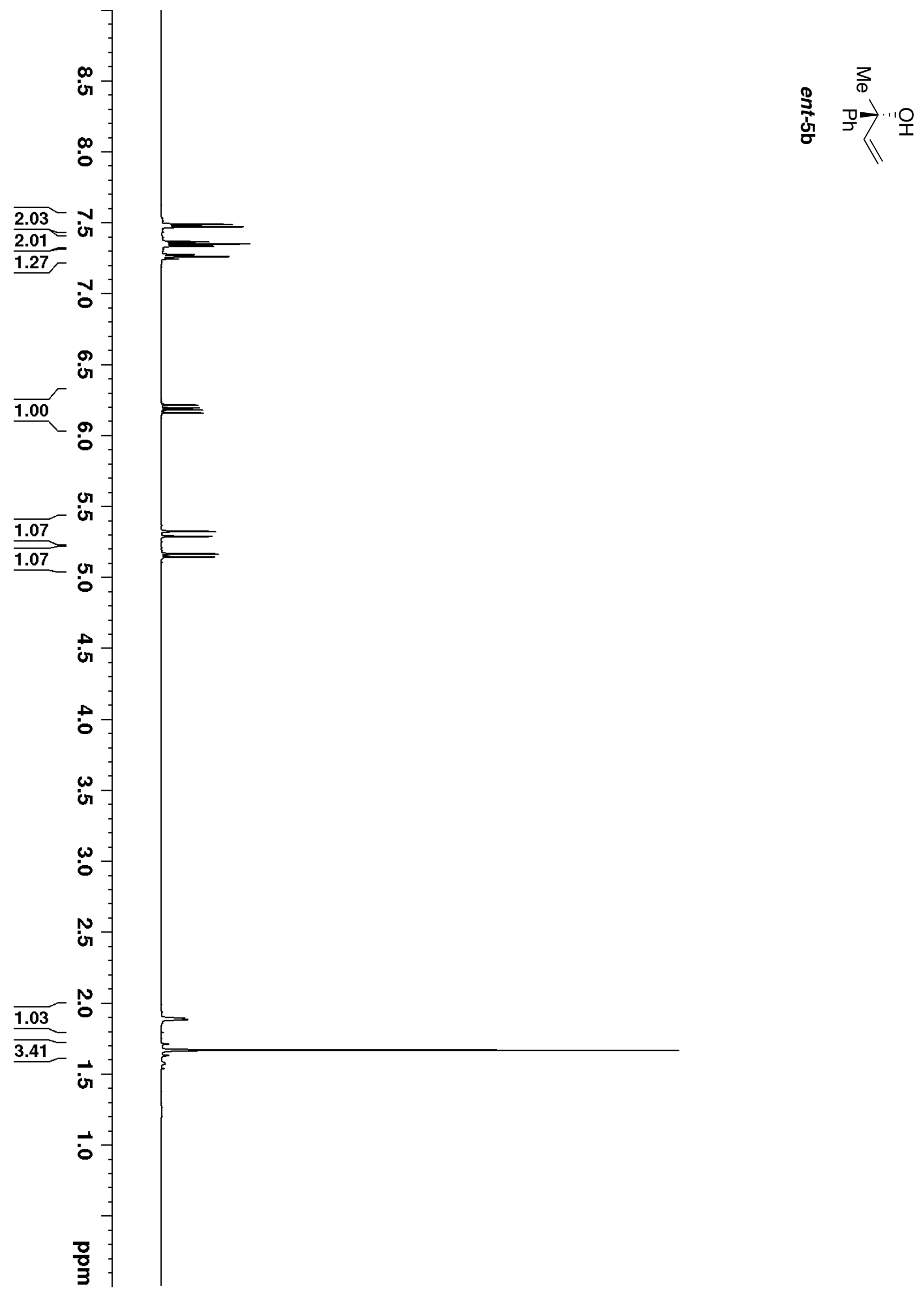



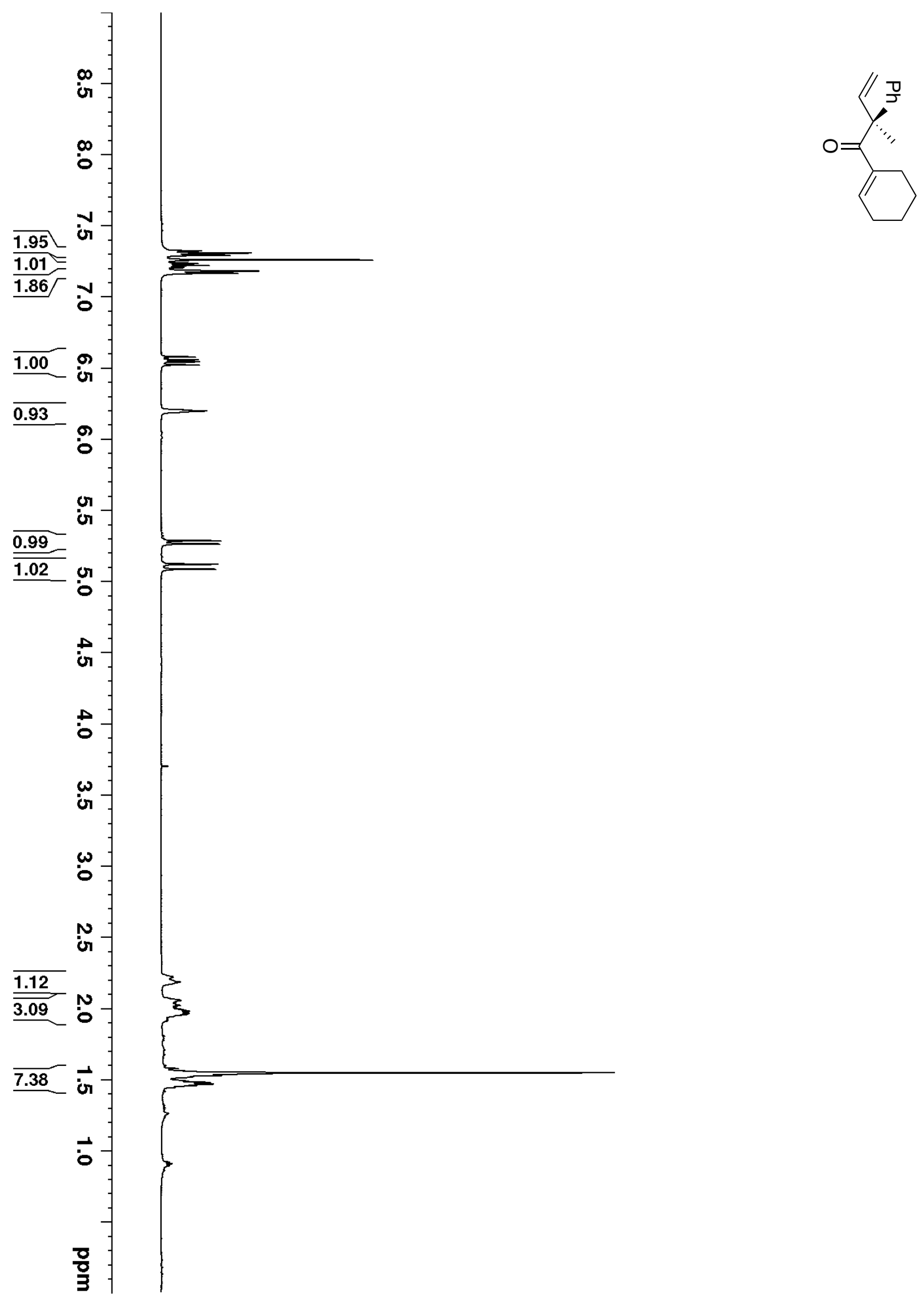

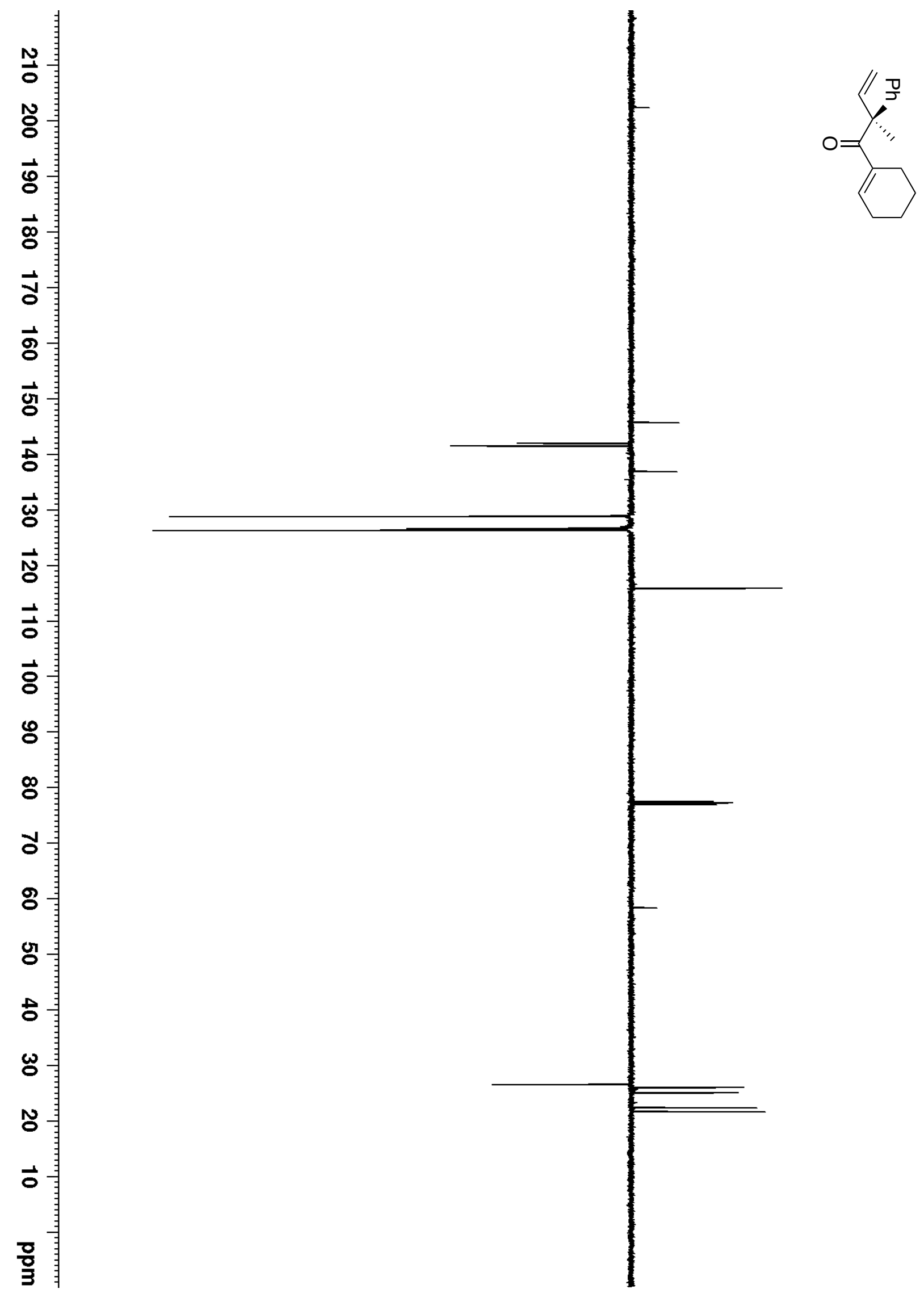

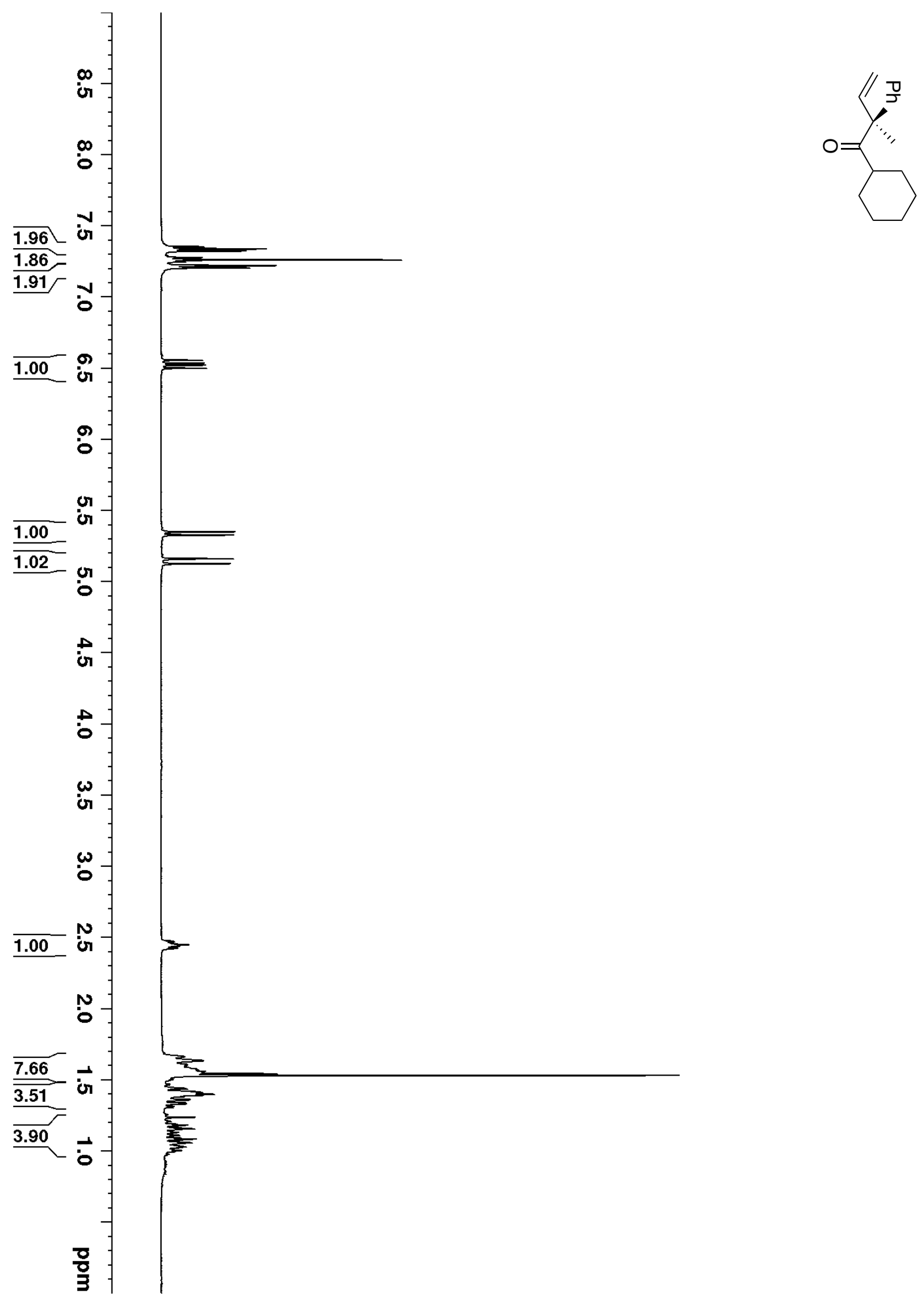


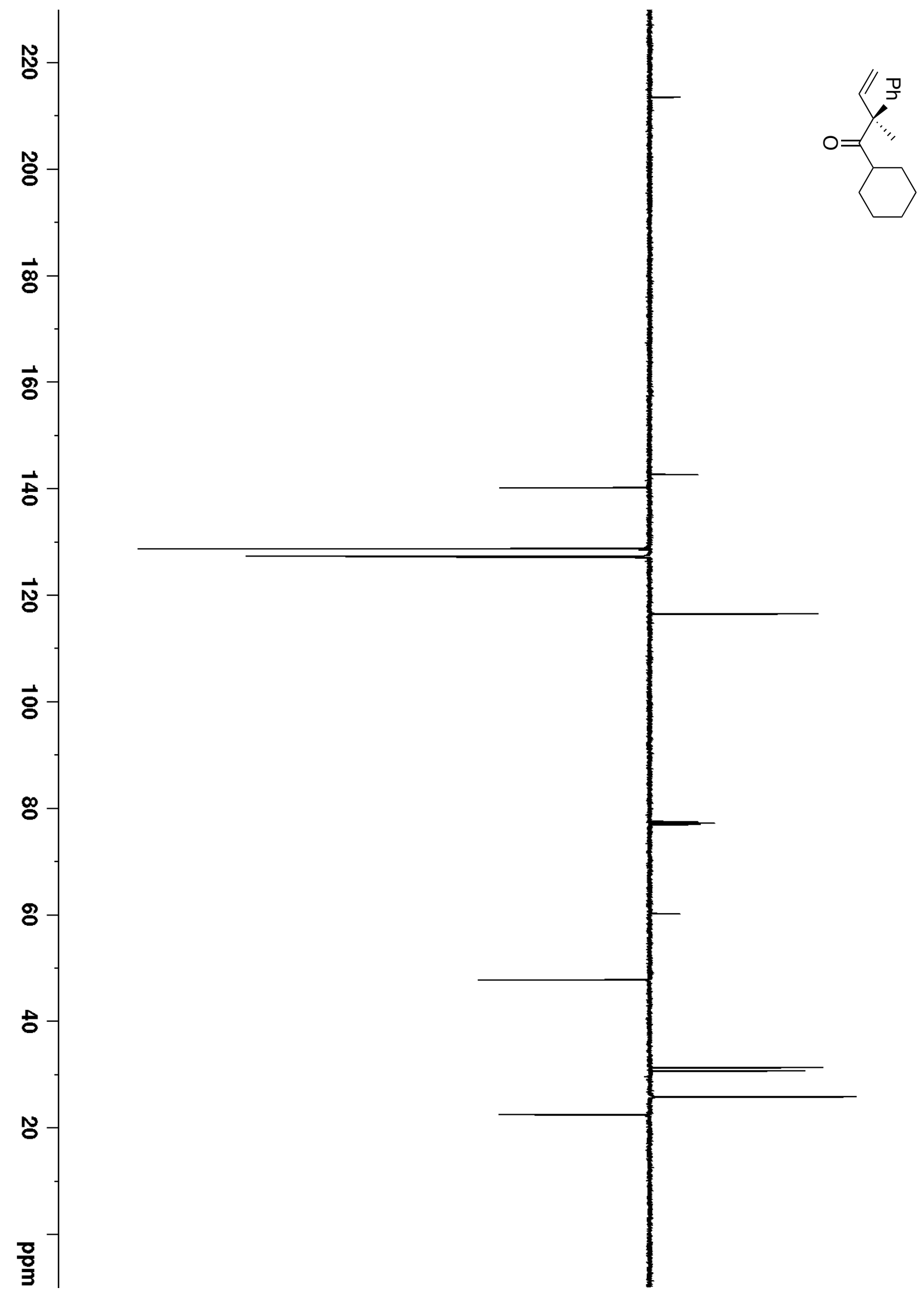



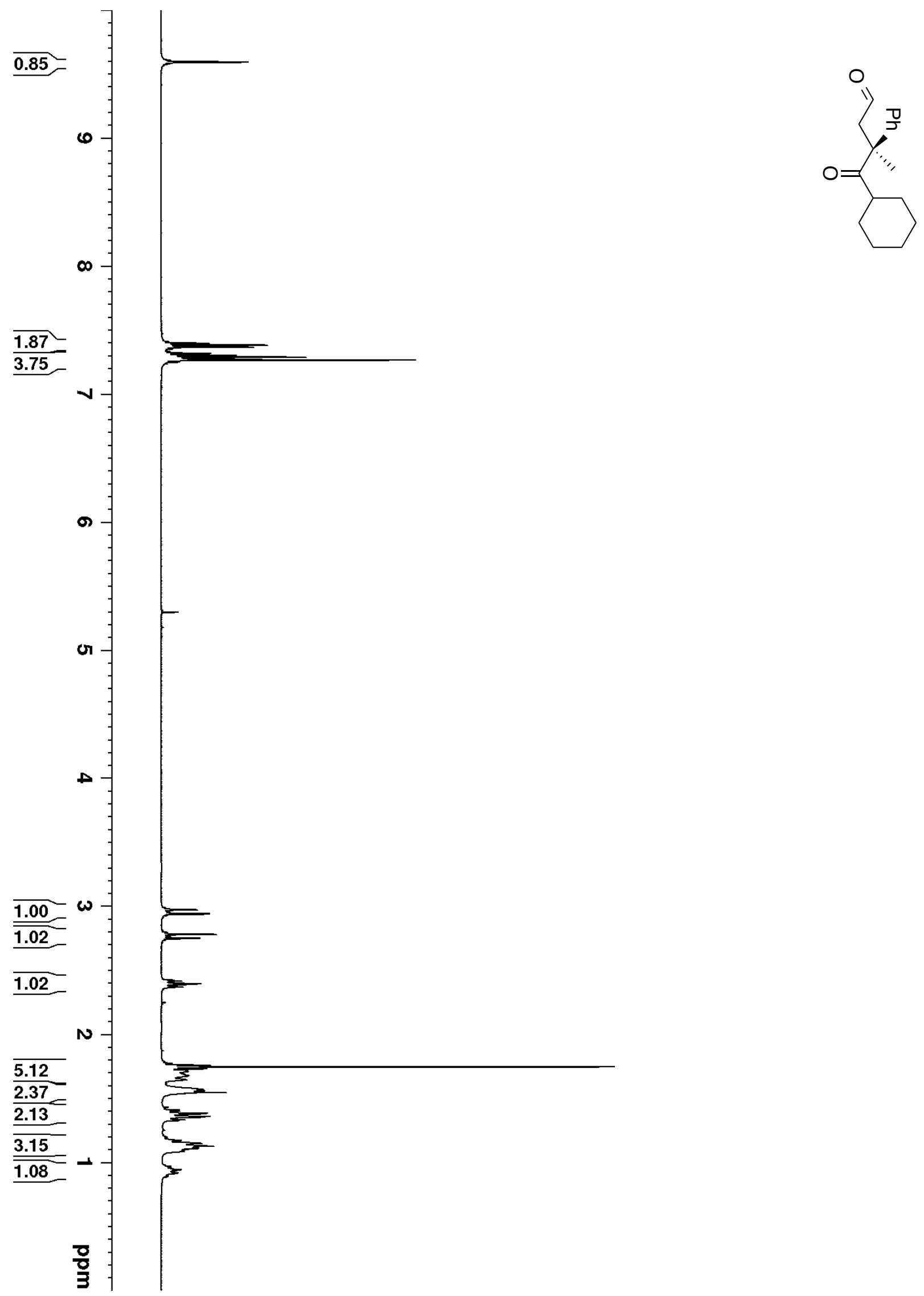


$$
H_{F}^{*}
$$



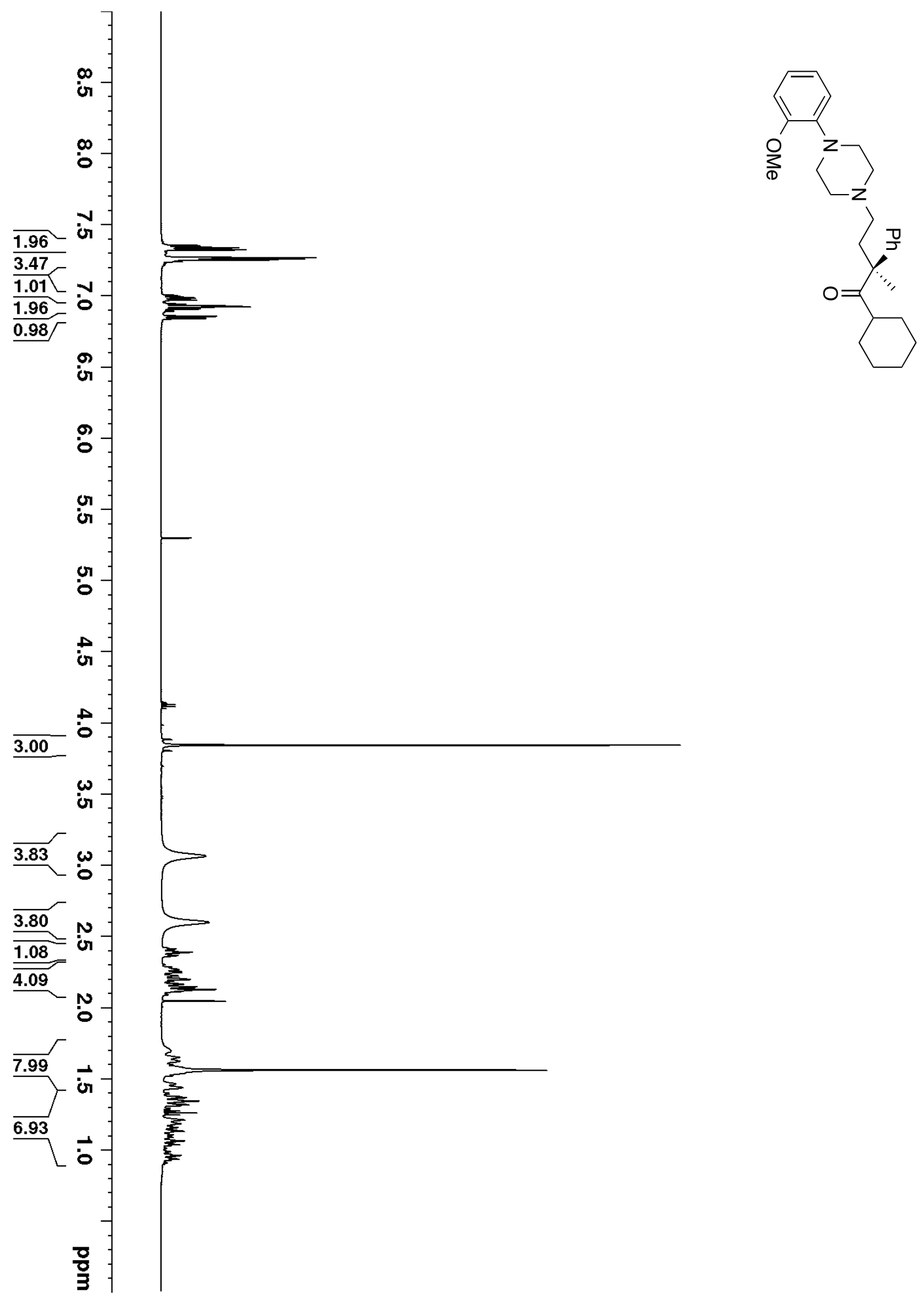


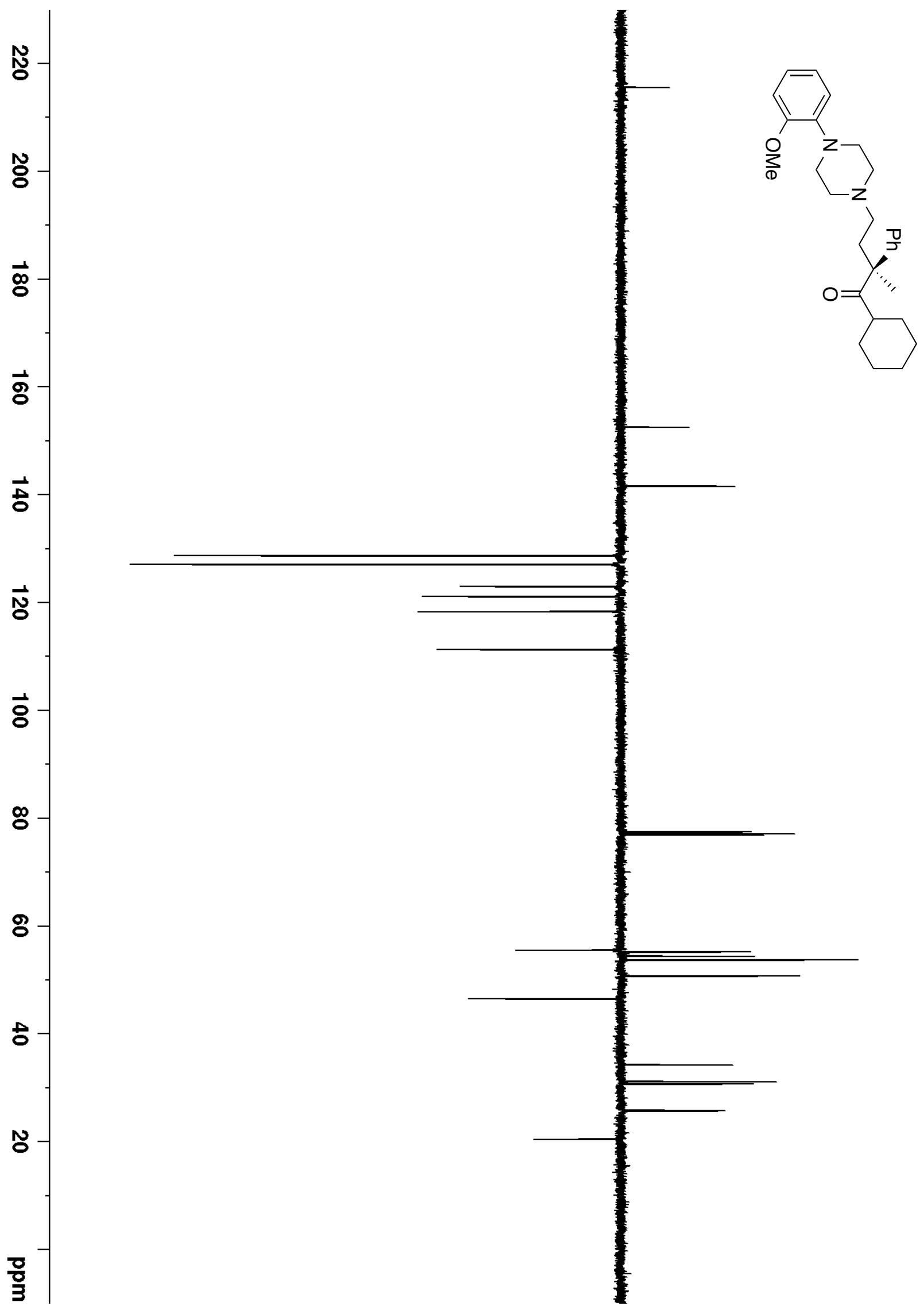




\section{HPLC Chromatograms}

\section{(R)-2-(4-Methoxyphenyl)but-3-en-2-ol (5a)}

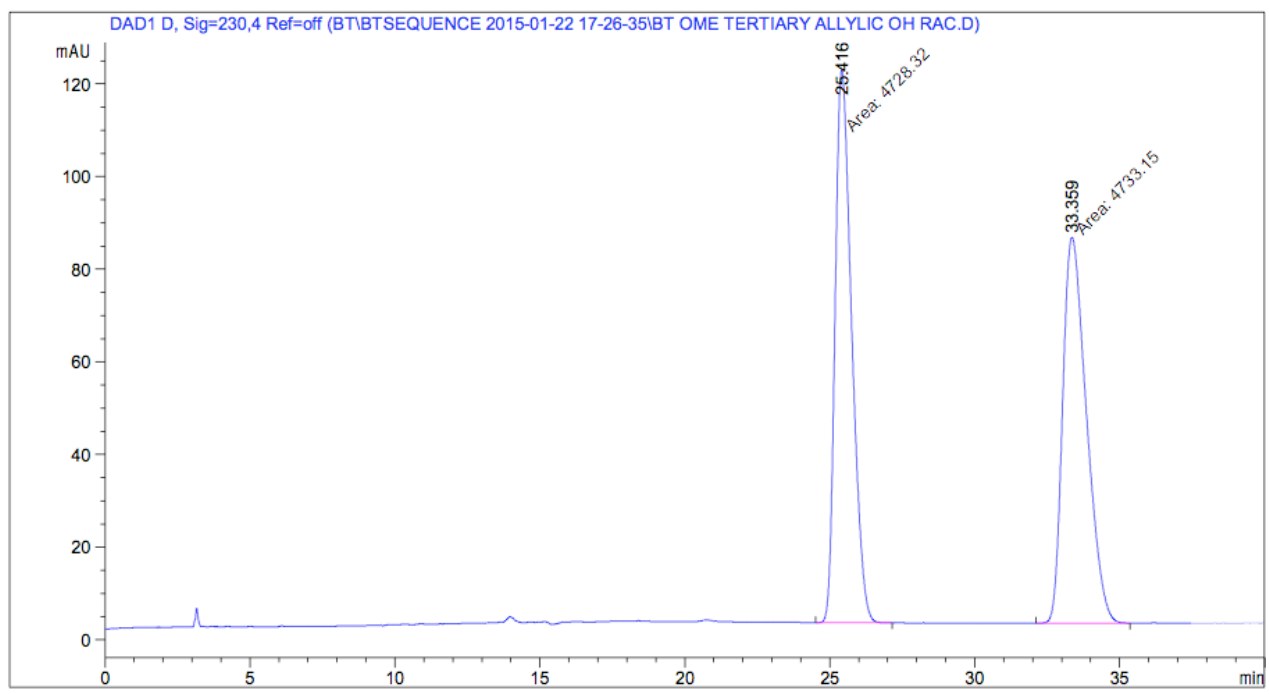

\begin{tabular}{|c|c|c|c|c|c|}
\hline $\begin{array}{c}\text { Peak } \\
\#\end{array}$ & $\begin{array}{l}\text { RetTime Type } \\
\text { [min] }\end{array}$ & $\begin{array}{l}\text { Width } \\
\text { [min] }\end{array}$ & $\begin{array}{c}\text { Area } \\
{[\mathrm{mAU} * \mathrm{~s}]}\end{array}$ & $\begin{array}{l}\text { Height } \\
\text { [mAU] }\end{array}$ & $\begin{array}{c}\text { Area } \\
\%\end{array}$ \\
\hline & & & & & \\
\hline 1 & $25.416 \mathrm{MM}$ & 0.6606 & 4728.32080 & 119.29335 & 49.9745 \\
\hline 2 & $33.359 \mathrm{MM}$ & 0.9469 & 4733.15137 & 83.30616 & 50.0255 \\
\hline
\end{tabular}

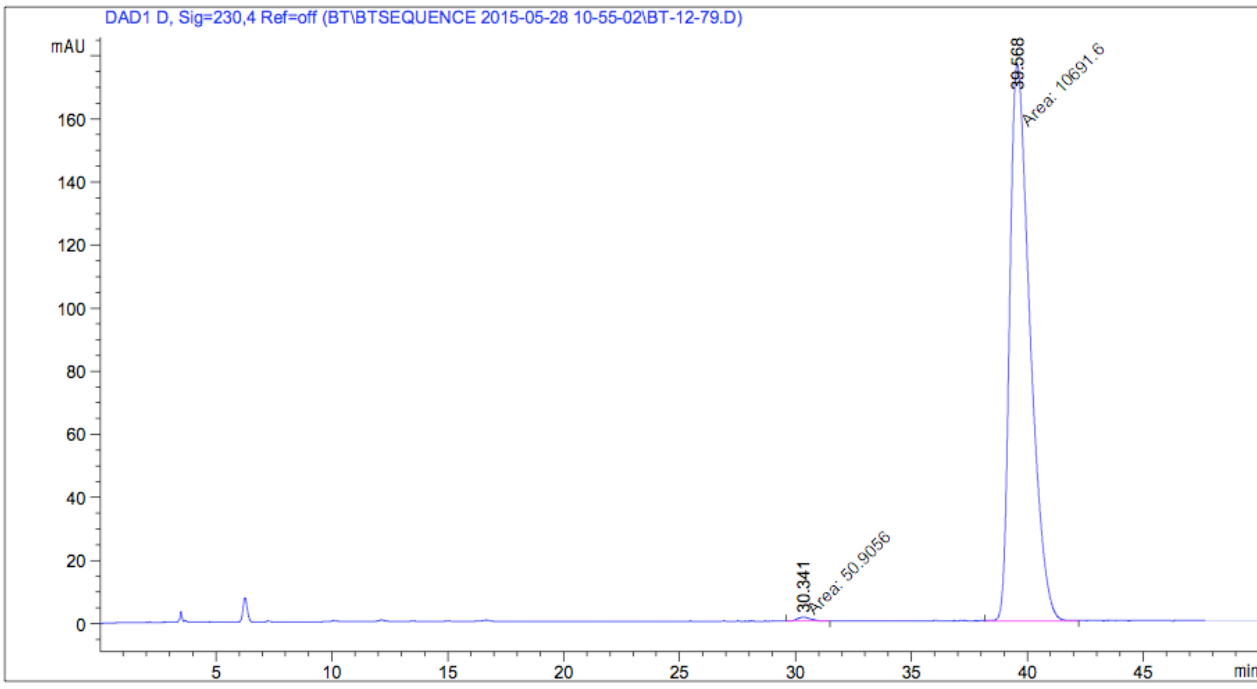

\begin{tabular}{|c|c|c|c|c|c|}
\hline $\begin{array}{c}\text { Peak } \\
\#\end{array}$ & $\begin{array}{l}\text { RetTime Type } \\
\text { [min] }\end{array}$ & $\begin{array}{l}\text { Width } \\
\text { [min] }\end{array}$ & $\begin{array}{c}\text { Area } \\
{[\mathrm{mAU} * \mathrm{~s}]}\end{array}$ & $\begin{array}{l}\text { Height } \\
\text { [mAU] }\end{array}$ & $\begin{array}{c}\text { Area } \\
\%\end{array}$ \\
\hline & & & 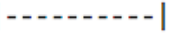 & $-\cdots$ & \\
\hline 1 & $30.341 \mathrm{MM}$ & 0.6676 & 50.90556 & 1.27084 & 0.4739 \\
\hline 2 & $39.568 \mathrm{MM}$ & 1.0110 & $1.06916 \mathrm{e} 4$ & 176.25594 & 99.5261 \\
\hline
\end{tabular}




\section{(R)-2-Phenylbut-3-en-2-ol (5b)}

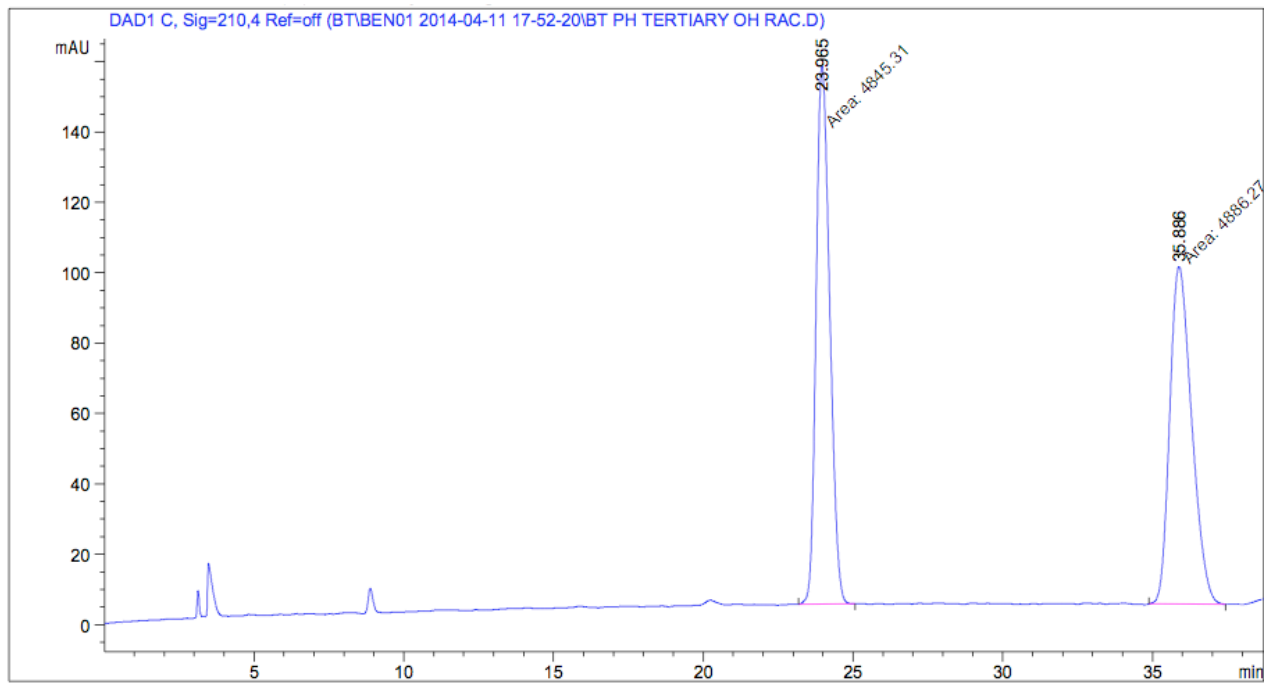

\begin{tabular}{cccccc}
$\begin{array}{c}\text { Peak RetTime Type } \\
\text { \# } \\
\text { [min] }\end{array}$ & $\begin{array}{c}\text { Width } \\
{[\mathrm{min}]}\end{array}$ & $\begin{array}{c}\text { Area } \\
{[\mathrm{mAU} \text { s }]}\end{array}$ & $\begin{array}{c}\text { Height } \\
{[\mathrm{mAU}]}\end{array}$ & $\begin{array}{c}\text { Area } \\
\%\end{array}$ \\
\hline 1 & $23.965 \mathrm{MM}$ & 0.5283 & 4845.31104 & 152.86560 & 49.7896 \\
2 & $35.886 \mathrm{MM}$ & 0.8492 & 4886.26904 & 95.89912 & 50.2104
\end{tabular}

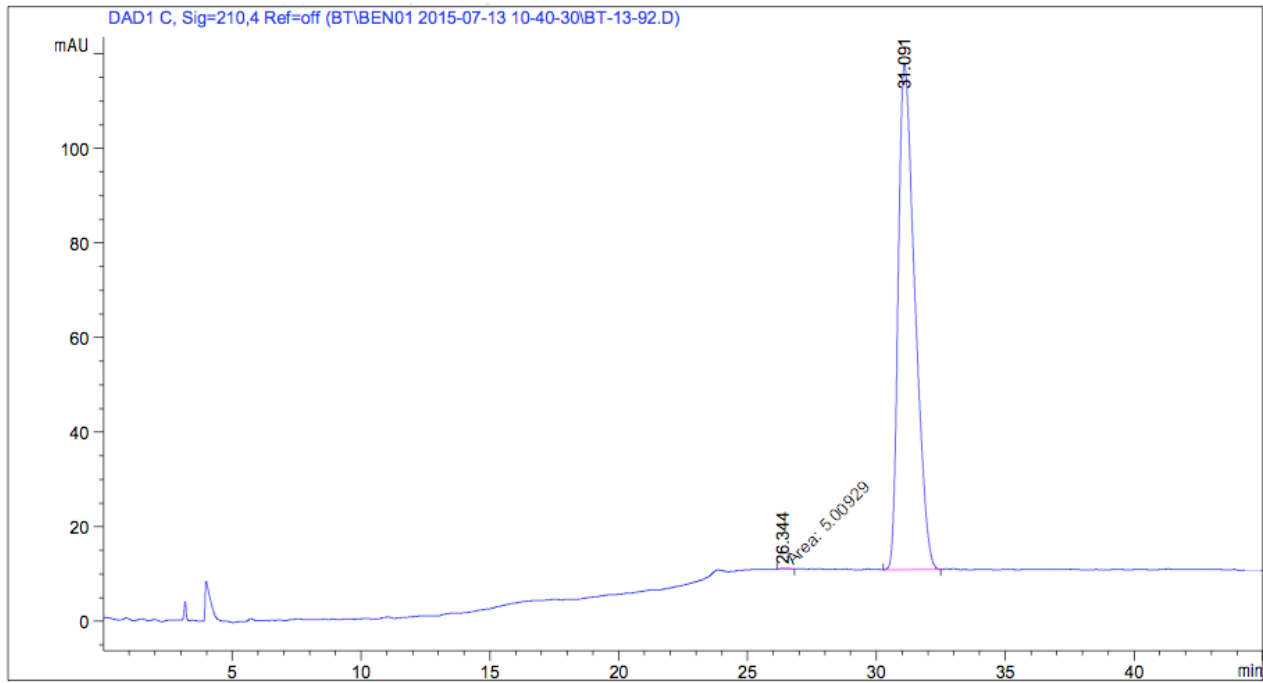

\begin{tabular}{cccccc}
$\begin{array}{c}\text { Peak RetTime Type } \\
\text { Width } \\
\text { [min] }\end{array}$ & $\begin{array}{c}\text { Area } \\
{[\mathrm{min}]}\end{array}$ & $\begin{array}{c}\text { Height } \\
{\left[\mathrm{mAU}{ }^{*} \mathrm{~s}\right]}\end{array}$ & $\begin{array}{c}\text { Area } \\
{[\mathrm{mAU}]}\end{array}$ & $\%$ \\
\hline 1 & 26.344 MM & 0.4528 & 5.00929 & $1.84398 \mathrm{e}-1$ & 0.1046 \\
2 & 31.091 BB & 0.6764 & 4784.08105 & 106.84220 & 99.8954
\end{tabular}




\section{(R)-2-(4-Fluorophenyl)but-3-en-2-ol (5c)}

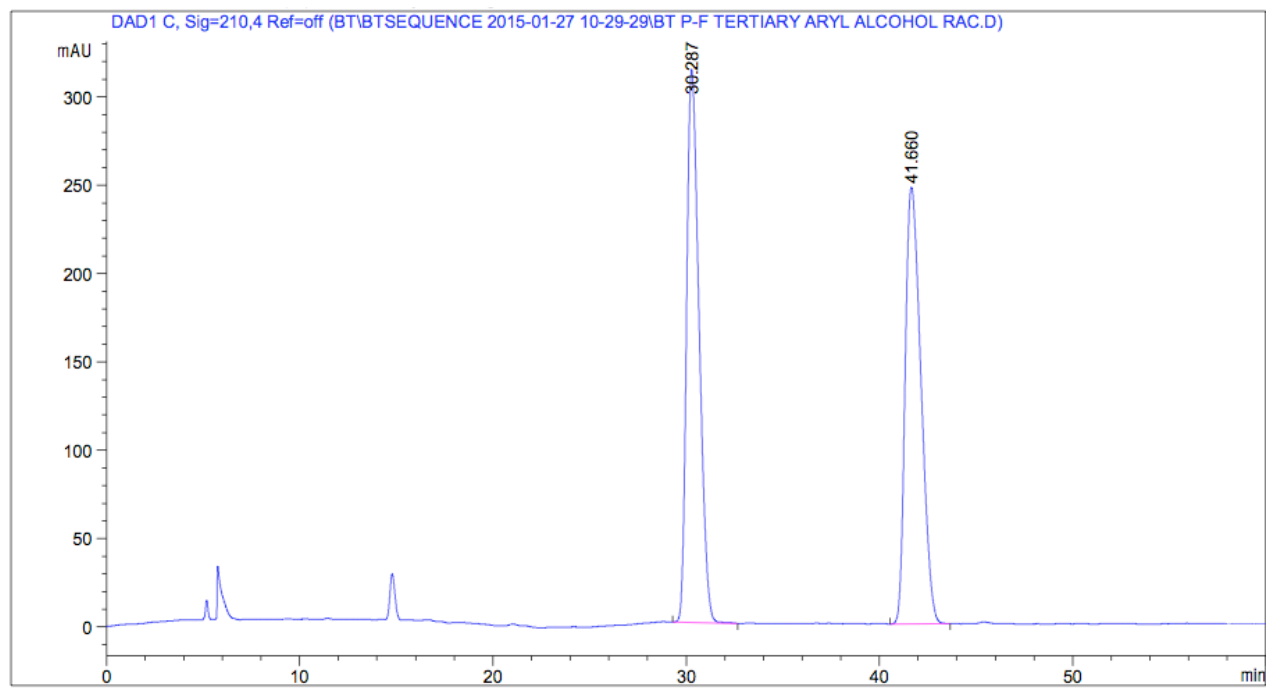

\begin{tabular}{cccccc}
$\begin{array}{c}\text { Peak RetTime Type } \\
\text { \# }\end{array} \quad \begin{array}{c}\text { Width } \\
{[\mathrm{min}]}\end{array}$ & $\begin{array}{c}\text { Area } \\
{[\mathrm{mAU} \text { * }]}\end{array}$ & $\begin{array}{c}\text { Height } \\
{[\mathrm{mAU}]}\end{array}$ & $\begin{array}{c}\text { Area } \\
\%\end{array}$ \\
\hdashline 1 & 30.287 BB & 0.6863 & $1.39675 \mathrm{e} 4$ & 313.19839 & 50.0331 \\
2 & 41.660 BB & 0.8906 & $1.39490 \mathrm{e} 4$ & 247.09360 & 49.9669
\end{tabular}

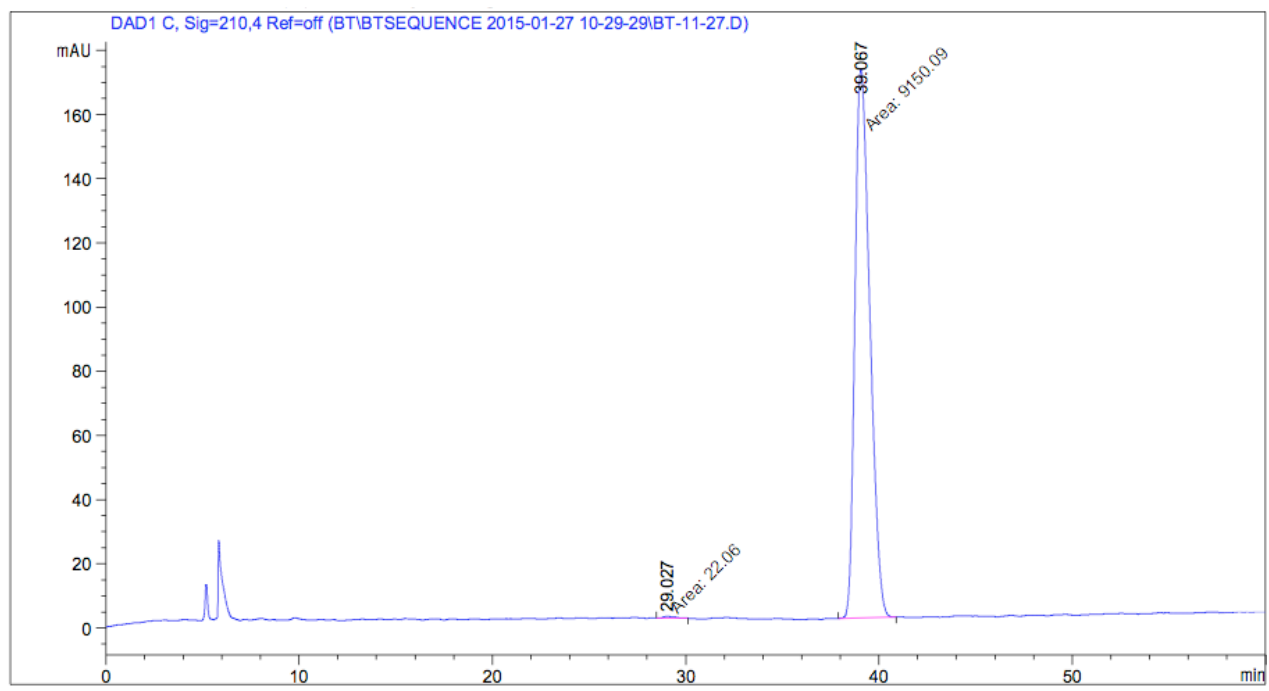

\begin{tabular}{|c|c|c|c|c|c|c|}
\hline $\begin{array}{c}\text { Peak } \\
\#\end{array}$ & $\begin{array}{c}\text { RetTime } \\
\text { [min] }\end{array}$ & Type & $\begin{array}{l}\text { Width } \\
\text { [min] }\end{array}$ & $\begin{array}{c}\text { Area } \\
{\left[\mathrm{mAU}^{*} \mathrm{~s}\right]}\end{array}$ & $\begin{array}{l}\text { Height } \\
\text { [mAU] }\end{array}$ & $\begin{array}{c}\text { Area } \\
\%\end{array}$ \\
\hline & & & & 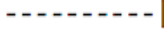 & 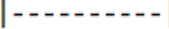 & \\
\hline 1 & 29. & MM & 0.6694 & 22.05997 & $5.49281 \mathrm{e}-1$ & 0.2405 \\
\hline 2 & 39.067 & MM & 0.8925 & 9150.09082 & 170.87444 & 99.7595 \\
\hline
\end{tabular}


(R,E)-3-(4-Methoxyphenyl)-3-methyl-8-phenylocta-1,5-dien-4-one (6a)
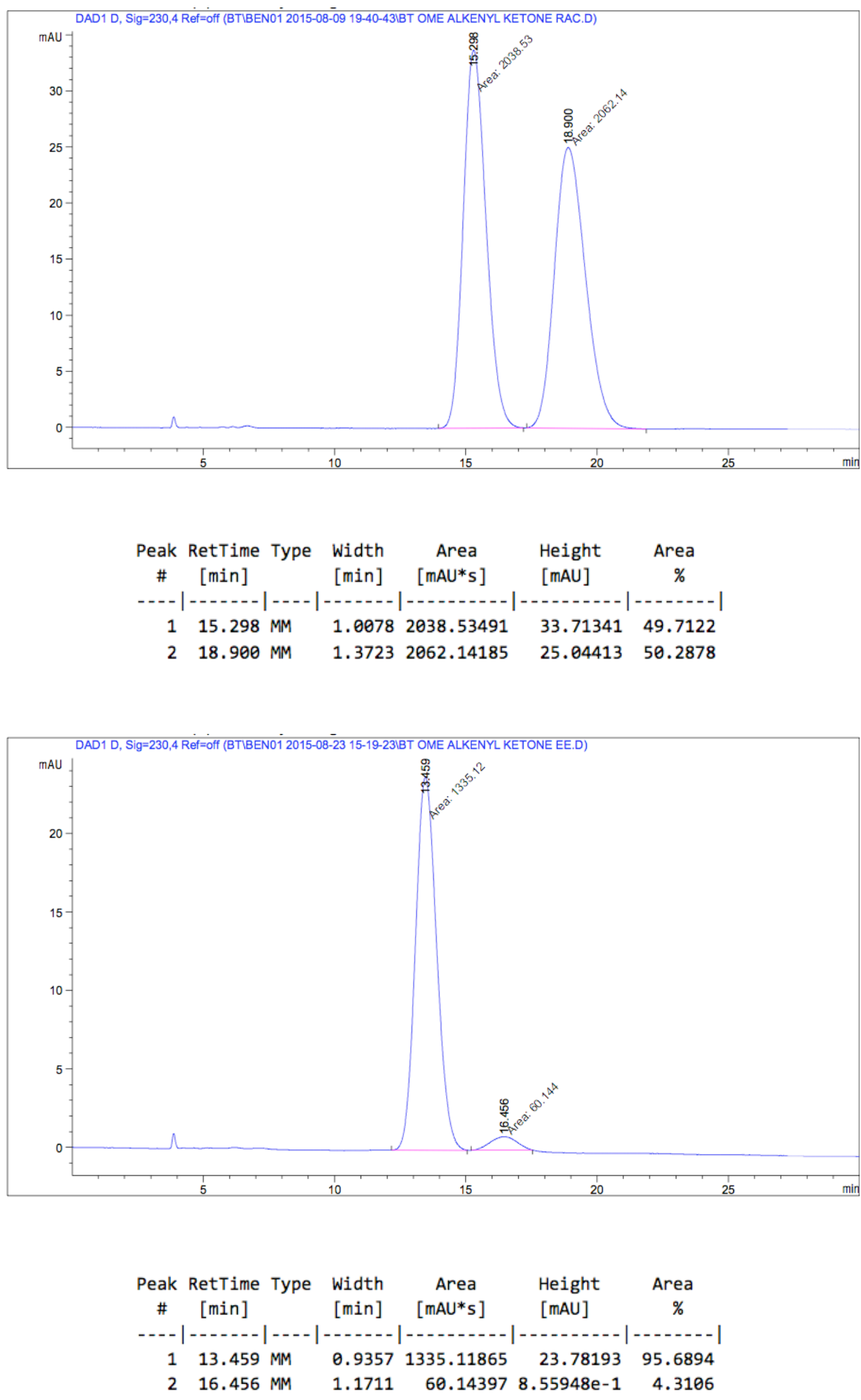


\section{$(R, E)-3-M e t h y l-3,8-d i p h e n y l o c t a-1,5-d i e n-4-o n e ~(6 b)$}

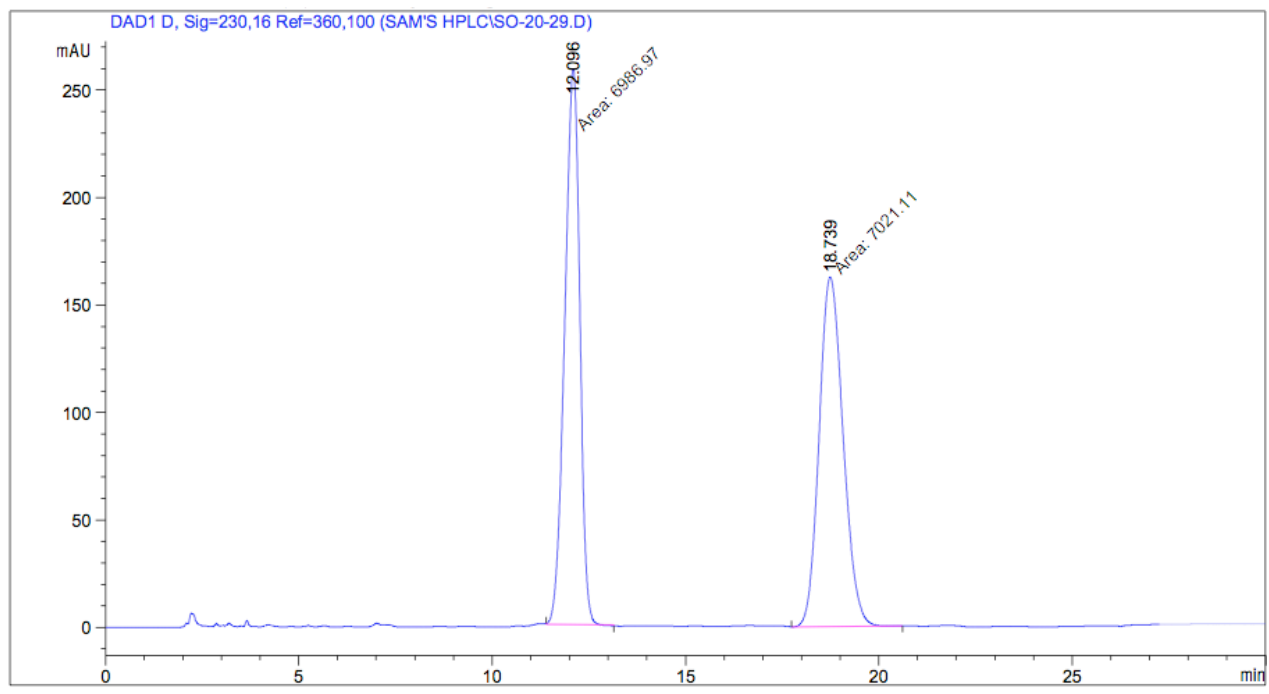

\begin{tabular}{|c|c|c|c|c|c|c|}
\hline $\begin{array}{c}\text { Peak } \\
\#\end{array}$ & $\begin{array}{c}\text { RetTime } \\
\text { [min] }\end{array}$ & Type & $\begin{array}{l}\text { Width } \\
\text { [min] }\end{array}$ & $\begin{array}{c}\text { Area } \\
{\left[\mathrm{mAU}^{*} \mathrm{~s}\right]}\end{array}$ & $\begin{array}{l}\text { Height } \\
\text { [mAU] }\end{array}$ & $\begin{array}{c}\text { Area } \\
\%\end{array}$ \\
\hline & & & & & & 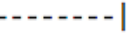 \\
\hline 1 & 12.096 & MM & 504 & 6986.97168 & 258.54358 & 49.8781 \\
\hline 2 & 18.739 & MM & 0.7188 & 7021.11279 & 162.79189 & 50.1219 \\
\hline
\end{tabular}

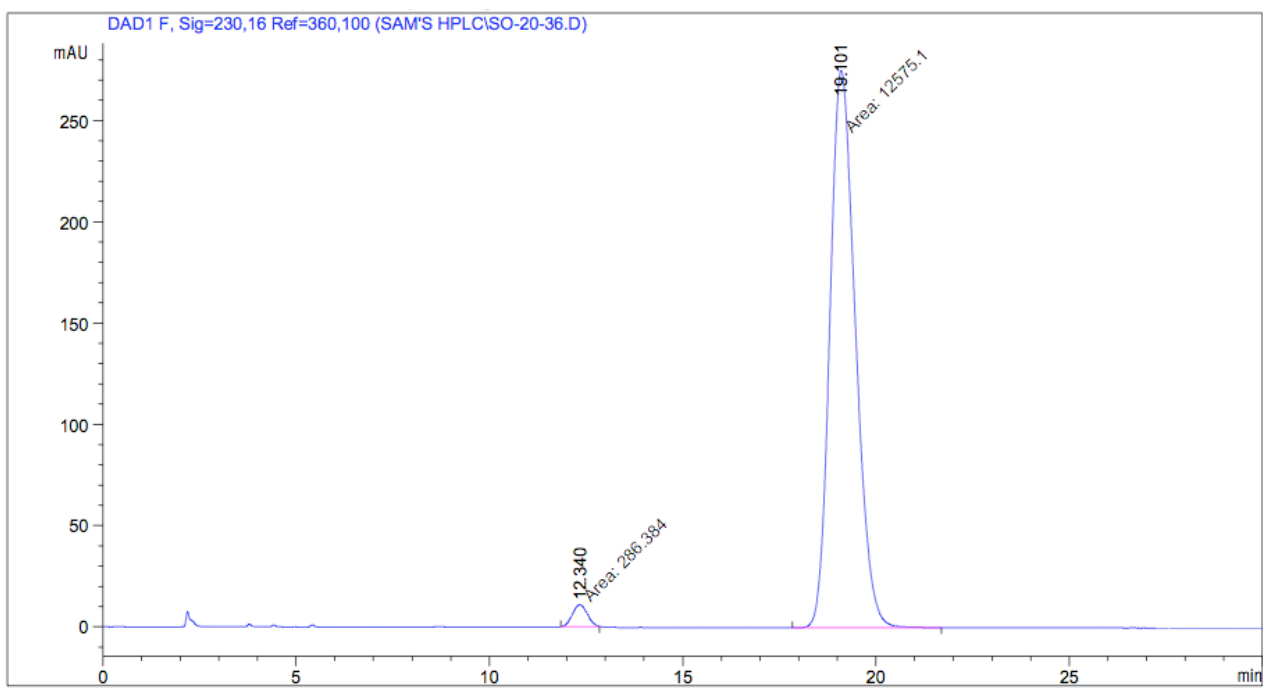

\begin{tabular}{|c|c|c|c|c|c|c|}
\hline $\begin{array}{c}\text { Peak } \\
\text { \# }\end{array}$ & $\begin{array}{c}\text { RetTime } \\
\text { [min] }\end{array}$ & Type & $\begin{array}{l}\text { Width } \\
\text { [min] }\end{array}$ & $\begin{array}{c}\text { Area } \\
{\left[\mathrm{mAU}^{*} \mathrm{~s}\right]}\end{array}$ & $\begin{array}{l}\text { Height } \\
{[\mathrm{mAU}]}\end{array}$ & $\begin{array}{c}\text { Area } \\
\%\end{array}$ \\
\hline & & & & 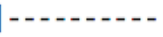 & ------1 & | \\
\hline 1 & 12.340 & MM & 0.4432 & 286.38367 & 10.76881 & 2.2267 \\
\hline 2 & 19.101 & MM & 0.7620 & $1.25751 \mathrm{e} 4$ & 275.03210 & 97.7733 \\
\hline
\end{tabular}




\section{$(R, E)-3-(4-F l u o r o p h e n y l)-3-m e t h y l-8-p h e n y l o c t a-1,5-d i e n-4-o n e ~(6 c)$}

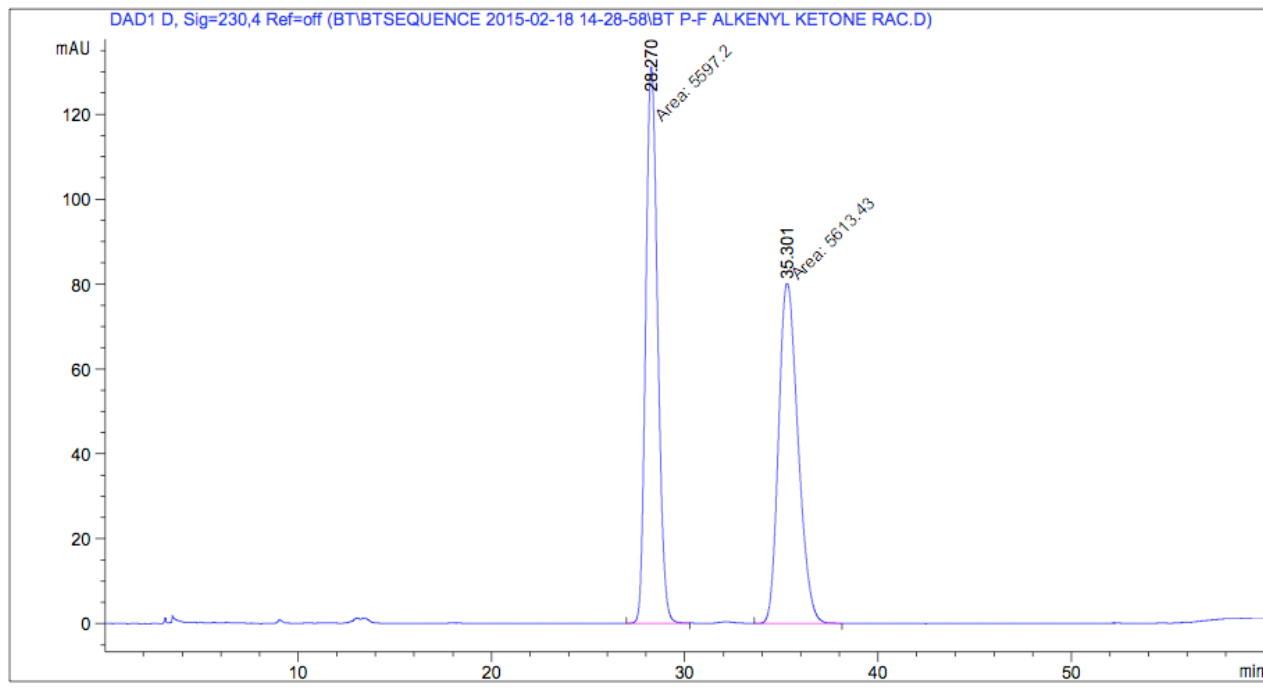

\begin{tabular}{|c|c|c|c|c|c|c|}
\hline $\begin{array}{c}\text { Peak } \\
\#\end{array}$ & $\begin{array}{c}\text { RetTime } \\
\text { [min] }\end{array}$ & Type & $\begin{array}{l}\text { Width } \\
\text { [min] }\end{array}$ & $\begin{array}{c}\text { Area } \\
{\left[\mathrm{mAU}^{*} \mathrm{~s}\right]}\end{array}$ & $\begin{array}{l}\text { Height } \\
\text { [mAU] }\end{array}$ & $\begin{array}{c}\text { Area } \\
\%\end{array}$ \\
\hline & & & & -1 & & \\
\hline 1 & 28.270 & MM & 0.7114 & 5597.20264 & 131.13286 & 49.9276 \\
\hline 2 & 35.301 & MM & 1.1677 & 5613.43457 & 80.11995 & 50.0724 \\
\hline
\end{tabular}

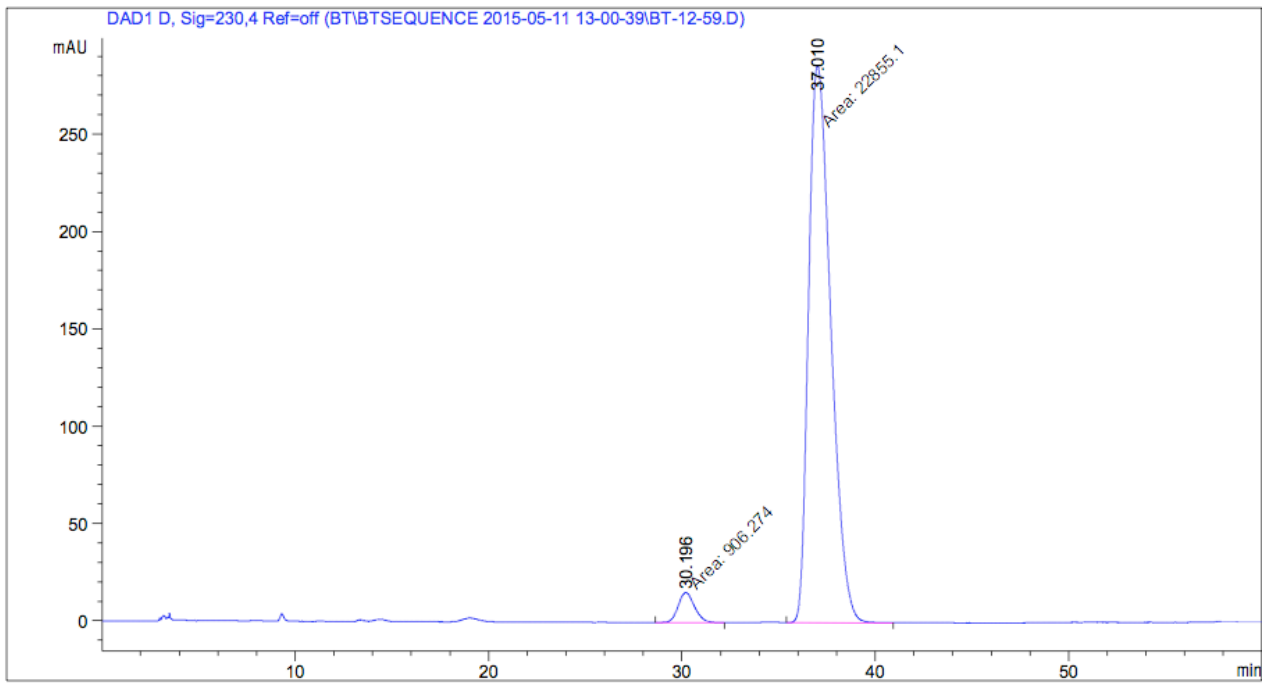

\begin{tabular}{|c|c|c|c|c|c|}
\hline $\begin{array}{c}\text { Peak } \\
\text { \# }\end{array}$ & $\begin{array}{l}\text { RetTime Type } \\
\text { [min] }\end{array}$ & $\begin{array}{l}\text { Width } \\
\text { [min] }\end{array}$ & $\begin{array}{c}\text { Area } \\
{[\mathrm{mAU} * \mathrm{~s}]}\end{array}$ & $\begin{array}{l}\text { Height } \\
{[\mathrm{mAU}]}\end{array}$ & $\begin{array}{c}\text { Area } \\
\%\end{array}$ \\
\hline 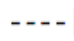 & & & -1 & 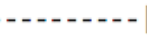 & | - - \\
\hline 1 & $30.196 \mathrm{MM}$ & 0.9811 & 906.27411 & 15.39553 & 3.8141 \\
\hline 2 & $37.010 \mathrm{MM}$ & 1.3307 & $2.28551 \mathrm{e} 4$ & 286.24542 & 96.1859 \\
\hline
\end{tabular}




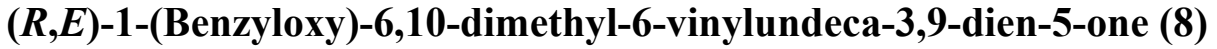

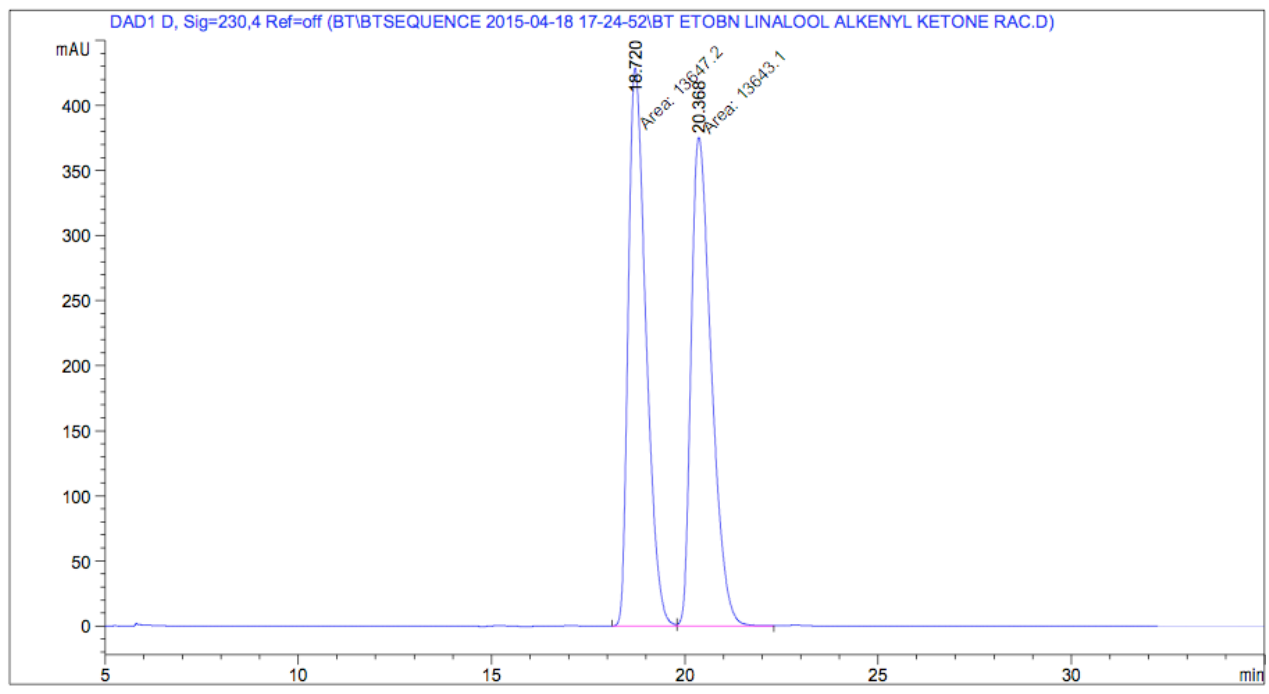

\begin{tabular}{cccccc}
$\begin{array}{c}\text { Peak RetTime Type width } \\
\text { \# } \\
{[\mathrm{min}]}\end{array}$ & $\begin{array}{c}\text { Area } \\
{[\mathrm{min}]}\end{array}$ & $\begin{array}{c}\text { Height } \\
{[\mathrm{mAU} \text { s }]}\end{array}$ & $\begin{array}{c}\text { Area } \\
{[\mathrm{mAU}]}\end{array}$ & $\%$ \\
\hline 1 & $18.720 \mathrm{MF}$ & 0.5302 & $1.36472 \mathrm{e} 4$ & 429.00137 & 50.0074 \\
2 & $20.368 \mathrm{FM}$ & 0.6054 & $1.36431 \mathrm{e} 4$ & 375.57159 & 49.9926
\end{tabular}

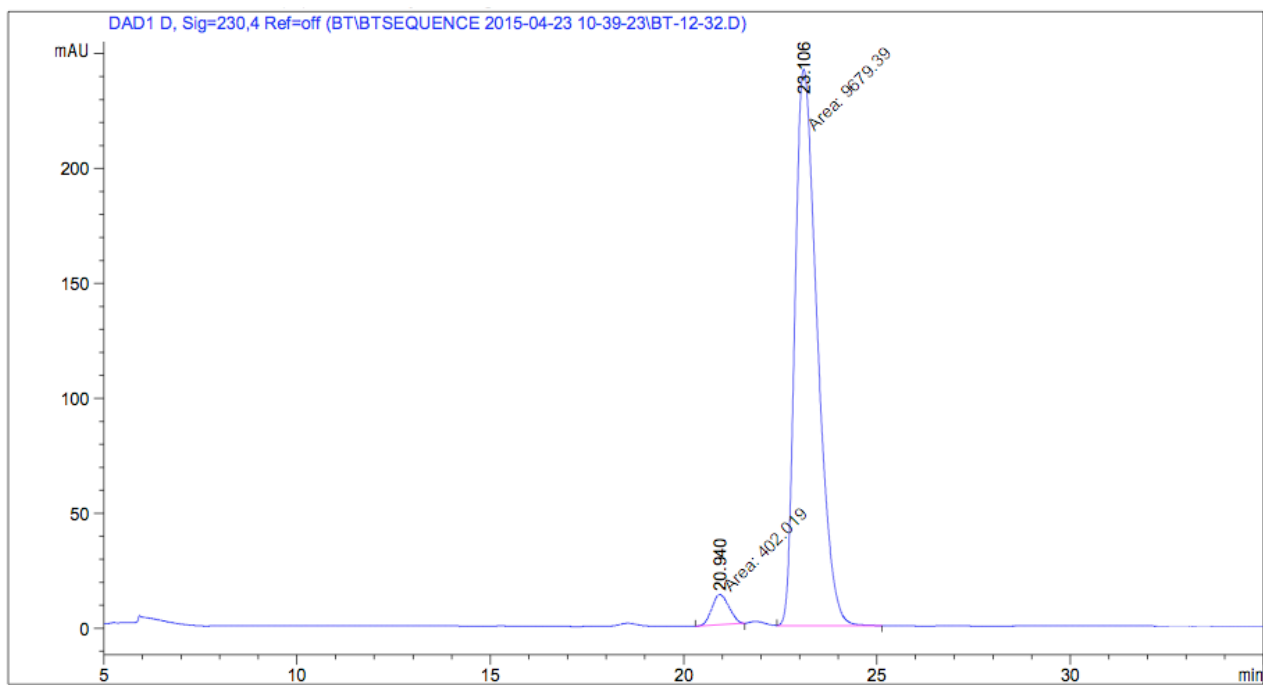

\begin{tabular}{cccccc}
$\begin{array}{c}\text { Peak RetTime Type } \\
\text { \# }\end{array}$ [min] & $\begin{array}{c}\text { Width } \\
{[\mathrm{min}]}\end{array}$ & $\begin{array}{c}\text { Area } \\
{[\mathrm{mAU} \text { s }]}\end{array}$ & $\begin{array}{c}\text { Height } \\
{[\mathrm{mAU}]}\end{array}$ & $\begin{array}{c}\text { Area } \\
\%\end{array}$ \\
\hline 1 & $20.940 \mathrm{MM}$ & 0.5134 & 402.01901 & 13.05061 & 3.9877 \\
2 & 23.106 MM & 0.6666 & 9679.38672 & 242.01460 & 96.0123
\end{tabular}




\section{(S)-2-Phenylbut-3-en-2-ol (ent-5b)}

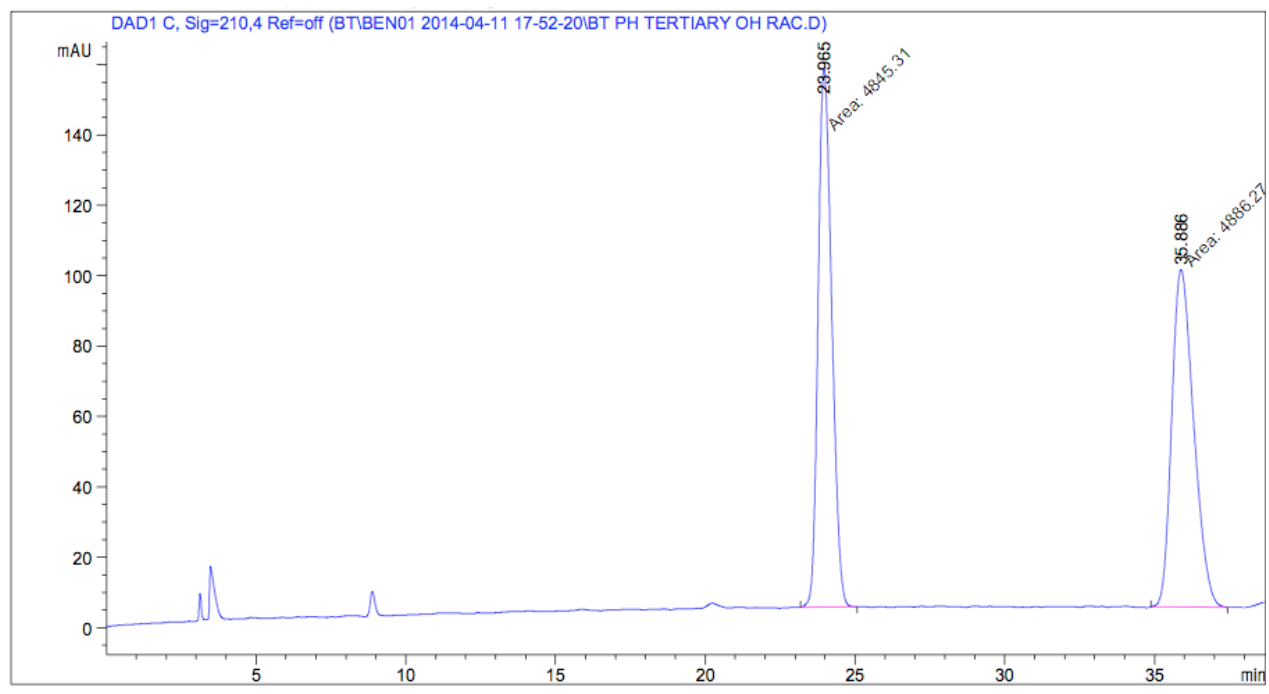

\begin{tabular}{|c|c|c|c|c|c|}
\hline $\begin{array}{c}\text { Peak } \\
\quad \#\end{array}$ & $\begin{array}{l}\text { RetTime Type } \\
\text { [min] }\end{array}$ & $\begin{array}{l}\text { Width } \\
\text { [min] }\end{array}$ & $\begin{array}{c}\text { Area } \\
{\left[\mathrm{mAU}^{*} \mathrm{~s}\right]}\end{array}$ & $\begin{array}{l}\text { Height } \\
{[\mathrm{mAU}]}\end{array}$ & $\begin{array}{c}\text { Area } \\
\%\end{array}$ \\
\hline & & & - & & -1 \\
\hline 1 & $23.965 \mathrm{MM}$ & 0.5283 & 4845.31104 & 152.86560 & 49.7896 \\
\hline 2 & $35.886 \mathrm{MM}$ & 0.8492 & 4886.26904 & 95.89912 & 50.2104 \\
\hline
\end{tabular}

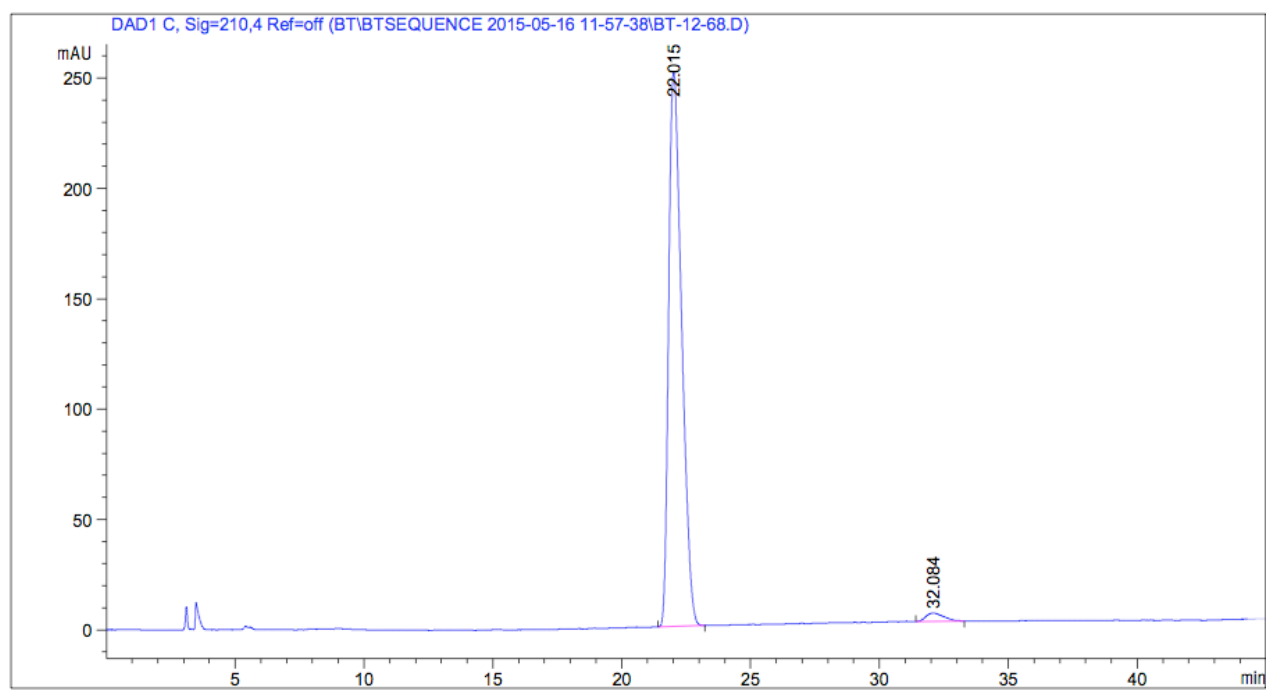

\begin{tabular}{|c|c|c|c|c|c|}
\hline $\begin{array}{c}\text { Peak } \\
\quad \#\end{array}$ & $\begin{array}{l}\text { RetTime Type } \\
\text { [min] }\end{array}$ & $\begin{array}{l}\text { Width } \\
\text { [min] }\end{array}$ & $\begin{array}{c}\text { Area } \\
{\left[\mathrm{mAU}^{*} \mathrm{~s}\right]}\end{array}$ & $\begin{array}{l}\text { Height } \\
\text { [mAU] }\end{array}$ & $\begin{array}{c}\text { Area } \\
\%\end{array}$ \\
\hline & & & . & & \\
\hline 1 & 22.015 BВ & 0.5355 & 8777.79883 & 251.28983 & 97.9395 \\
\hline 2 & $32.084 \mathrm{BB}$ & 0.5864 & 184.67134 & 3.83111 & 2.0605 \\
\hline
\end{tabular}




\section{(S)-1-Cyclohexenyl-2-methyl-2-phenylbut-3-en-1-one}

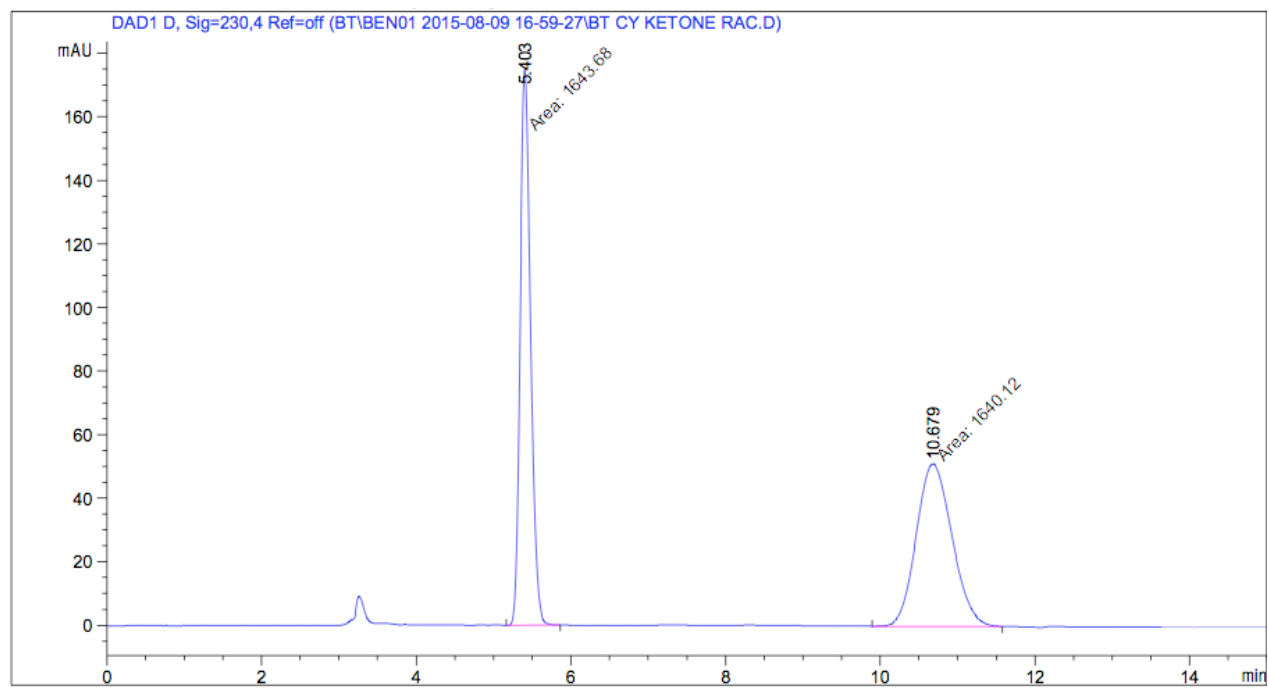

\begin{tabular}{cccccc}
$\begin{array}{c}\text { Peak RetTime Type } \\
\text { \# }\end{array}$ [min] & $\begin{array}{c}\text { Width } \\
{[\text { min] }}\end{array}$ & $\begin{array}{c}\text { Area } \\
{[\text { mAU*s }]}\end{array}$ & $\begin{array}{l}\text { Height } \\
{[\text { mAU] }}\end{array}$ & $\begin{array}{c}\text { Area } \\
\%\end{array}$ \\
\hdashline 1 & $5.403 \mathrm{MM}$ & 0.1565 & 1643.67627 & 175.09285 & 50.0542 \\
2 & $10.679 \mathrm{MM}$ & 0.5344 & 1640.11963 & 51.15470 & 49.9458
\end{tabular}

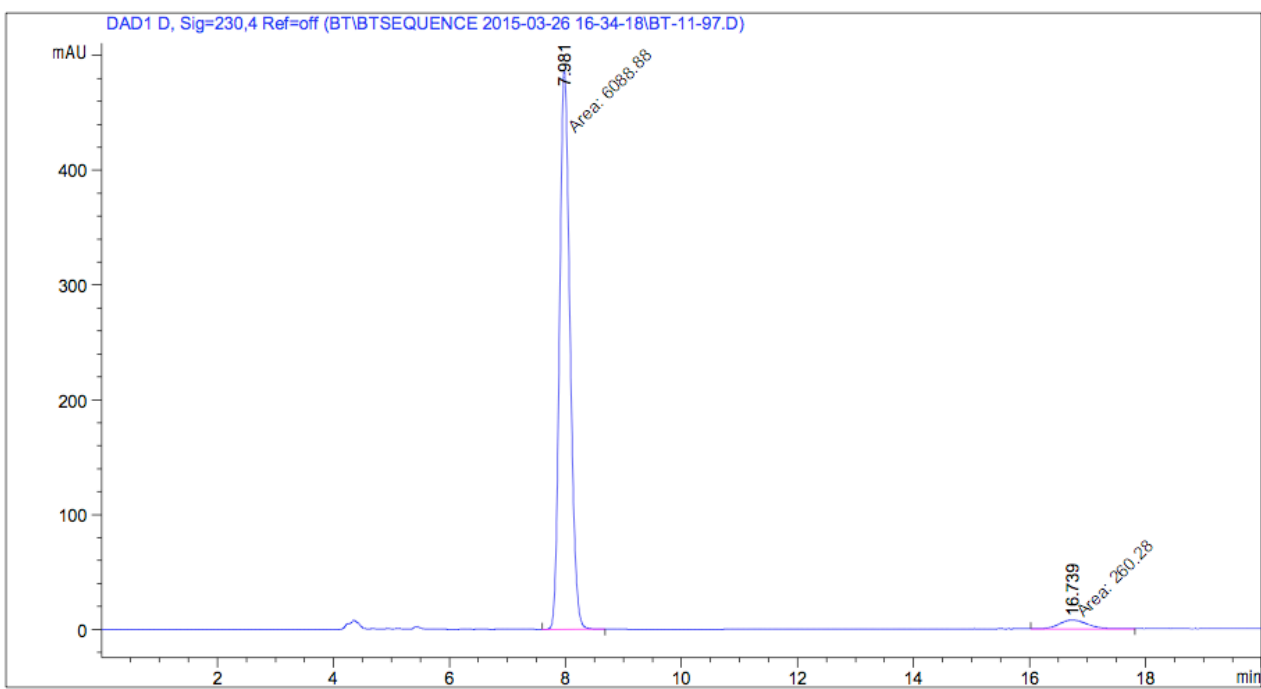

\begin{tabular}{|c|c|c|c|c|c|}
\hline $\begin{array}{c}\text { Peak } \\
\#\end{array}$ & $\begin{array}{l}\text { RetTime Type } \\
\text { [min] }\end{array}$ & $\begin{array}{l}\text { Width } \\
\text { [min] }\end{array}$ & $\begin{array}{c}\text { Area } \\
{\left[\mathrm{mAU}^{*} \mathrm{~s}\right]}\end{array}$ & $\begin{array}{l}\text { Height } \\
\text { [mAU] }\end{array}$ & $\begin{array}{c}\text { Area } \\
\%\end{array}$ \\
\hline & & & 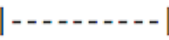 & & - \\
\hline 1 & $7.981 \mathrm{MM}$ & 0.2085 & 6088.88330 & 486.74936 & 95.9006 \\
\hline 2 & $16.739 \mathrm{MM}$ & 0.5602 & 260.28049 & 7.74406 & 4.0994 \\
\hline
\end{tabular}

